\title{
Regional Health Status Indicators Boston Massachusetts
}

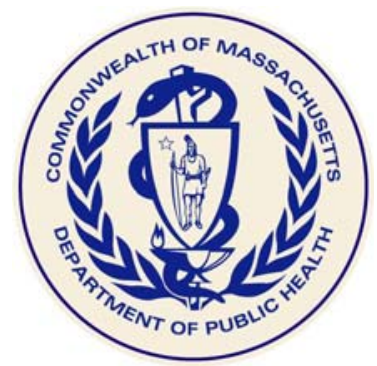

Deval L. Patrick, Governor

Timothy P. Murray, Lieutenant Governor

Judyann Bigby, MD, Secretary of Health and Human Services John Auerbach, Commissioner, Department of Public Health

Jerry O'Keefe, Associate Commissioner

Health Information, Statistics, Research, and Evaluation Bureau

Bruce Cohen, Director

Division of Research and Epidemiology

Massachusetts Department of Public Health

June 2007 


\section{ACKNOWLEDGEMENTS}

This report was prepared by Isabel Caceres, MS, Malena Orejuela-Hood, MPH, and James West, PhD of the Division of Research and Epidemiology and by Lauren Wooley, MPH, of the Health Survey Program, Center for Health Information, Statistics, Research, and Evaluation.

Special thanks go to Bruce Cohen, PhD, Director of the Division or Research and Epidemiology, and to all of the DPH Bureaus who have contributed to this report.

To obtain additional copies of this report, contact:

Massachusetts Department of Public Health

Health Information, Statistics, Research and Evaluation Bureau

250 Washington Street, $6^{\text {th }}$ Floor

Boston, MA 02108

This and other Department of Public Health publications can be downloaded from the following website:

http://www.mass.gov/dph/resep

To obtain more information on the health of Massachusetts and other Department of Public Health data:

Register for the Department's free, Internet-based public health information service, MassCHIP, via the website at:

http://masschip.state.ma.us

Or call 888-MAS-CHIP (toll free in MA) or 617-624-5629 


\section{TABLE OF CONTENTS}

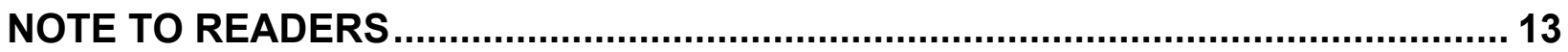

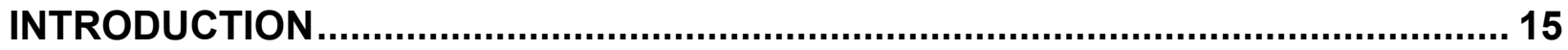

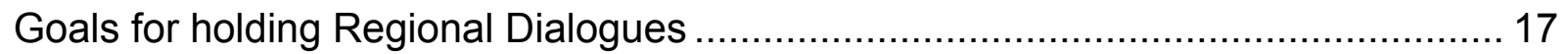

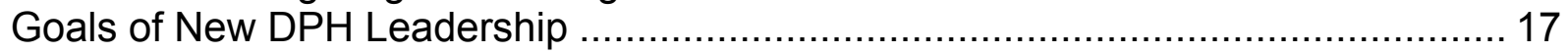

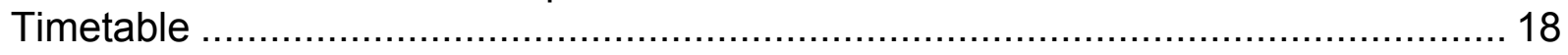

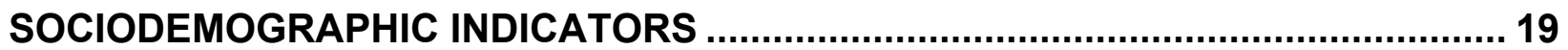

Executive Office of health and Human Services Regions ..................................... 21

A few comments about the Limitations of this presentation.................................. 21

Population in Cities of Boston Region: 2005 ............................................... 22

Race and Ethnic Breakdown of Population, Boston Region and Massachusetts:

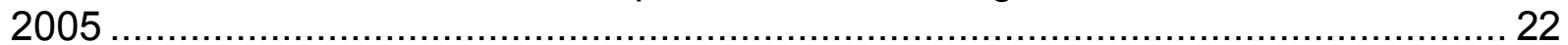

Race and Ethnic Breakdown of Population, Cities in Boston Region and

Massachusetts: 2005 .............................................................................. 23

Population by Age Group, Cities in Boston Region and Massachusetts: 2005 ........ 23

Language Spoken at Home, Cities in Boston Region and Massachusetts: 2000 ...... 24

Spanish Spoken at Home, Boston Region: 2000 .......................................... 24

Socio-Demographic Indicators Cities in Boston Region and Massachusetts: 2000 .. 25

Highest and Lowest per Capita Income Communities in Boston Region: 2000......... 25

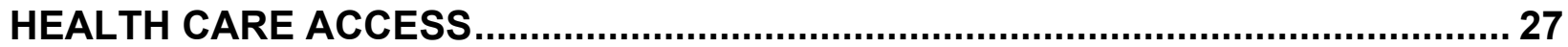

Adults who Have No Health Insurance by EOHHS Region, Massachusetts: 2005 ... 29

Adults who Have No Health Insurance by Race and Ethnicity Boston Region,

Massachusetts: 1999-2005 ……............................................................ 29

Health Insurance Access, Boston Region and Massachusetts: 2005 ..................... 30

Summary Indicators for Health Insurance by EOHHS Regions, Massachusetts:

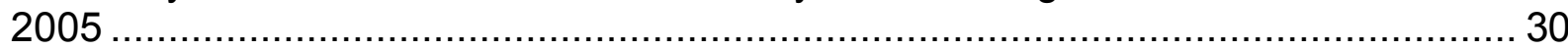

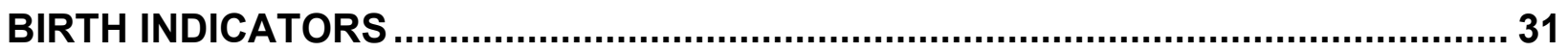

Infant Mortality Rate (IMR) Boston Region and Massachusetts: 1990-2005 ............. 33

Infant Mortality Rates by EOHHS Region and Massachusetts: 2003-2005 .............. 33

Infant Mortality Rates by Race/Ethnicity, Boston Region and Massachusetts: 2003-

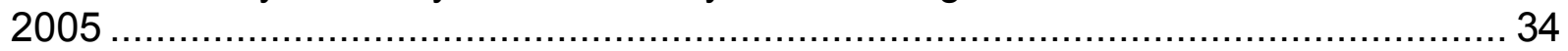

Low Birthweight by Boston Region, Massachusetts: 1990-2005 ......................... 34

Low Birthweight Cities in Boston Region and Massachusetts: 1990-2005 ............... 35

Low Birthweight by EOHHS Region, Massachusetts: 2005 ................................ 35

Low Birthweight by Cities in Boston Region and Massachusetts: 2005 .............................36

Breastfeeding by EOHHS Region, Massachusetts: 2005.................................. 36

Teen Birth Rates, Boston Region and Massachusetts: 1990-2005 …................... 37

Teen Birth Rates Cities in Boston Region and Massachusetts: 1990-2005 .............. 37

Teen Birth Rates, Cities in Boston Region and Massachusetts: 2003-2005 ............. 38

Teen Birth Rates by EOHHS Regions, Massachusetts: 2005 ….......................... 38

Teen Birth Rates by EOHHS Region, Massachusetts: 2005 ................................. 39 
Teen Birth Rates by City/Town, Boston Region: 2005

Teen Birth Rates by Race and Ethnicity, Boston Region and Massachusetts: 200540 Teen Birth Rates by Race and Ethnicity, Cities in Boston Region and Massachusetts: 2005 ...... 40

Adequate Prenatal Care, Boston Region and Massachusetts: 1990-2005.............. 41

Adequate Prenatal Care by EOHHS Regions, Massachusetts: 2005 ..................... 41

Public Source of Payment for Prenatal Care, Boston Region and Massachusetts: 1990- 2005

Public Source of Payment for Prenatal Care Boston Region and Massachusetts:

2005

Public Source of Payment for Prenatal Care, Cities in Boston Region and

Massachusetts: 2003-2005

Infants Delivered by C-Section, Boston Region and Massachusetts: 1990-2005 ..... 43

Infants Delivered by C-Section by EOHHS Regions, Massachusetts: 2005 ...................44

Infants Delivered by C-Section by EOHHS Regions, Massachusetts: 2005 ...................44

Percent of Cesarean Deliveries by City/Town, Boston Region: 2005 ..................... 45

Summary of Birth Indicators by EOHHS Regions, Massachusetts: 2005 ................. 45

Summary of Birth Indicators by EOHHS Regions, Massachusetts: 2005 ................. 46

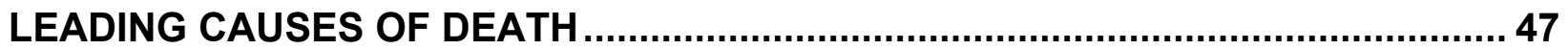

Leading Causes of Death, Boston Region and Massachusetts: 2005.................... 49

CHRONIC DISEASE OUTCOMES AND OVERWEIGHT/OBESITY ........................51

Thresholds for Defining Overweight and Obesity ............................................. 53

Adults who are Overweight by EOHHS Region, Massachusetts: 1995 \& 2005........ 53

Adults who are Overweight by Race and Ethnicity, Boston Region: 2005................ 54

Adults who are Obese by EOHHS Region, Massachusetts : 1995 \& 2005 ............... 54

Adults who Participate in Regular Physical Activity by EOHHS Region,

Massachusetts: 2005 .................................................................................... 55

Adults who have $5+$ Servings of Fruit or Vegetables by EOHHS Region,

Massachusetts : 2005 .......................................................................... 55

Adults who had their Cholesterol Checked in Past 5 Years by EOHHS Region,

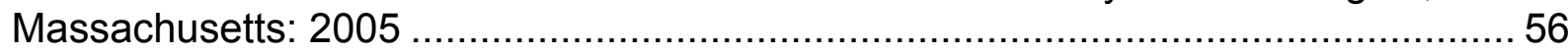

Adults who were Told by Health Care Provider that they had High Cholesterol, by

EOHHS Region, Massachusetts: 2005 ....................................................... 56

Adults who were Told by a Health Care Provider they have High Blood Pressure, by

EOHHS Region, Massachusetts: 2005 .........................................................5 57

Adults who take Medicine for High Blood Pressure, by EOHHS Region,

Massachusetts : 2005

Hospital Discharge Rates for Hypertension by EOHHS Region, Massachusetts: 2003-

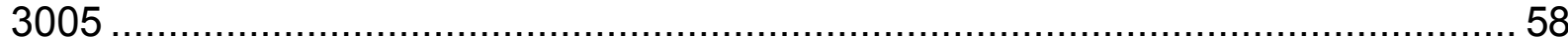

Hospital Discharge Rates for Hypertension by Cities in Boston Region and

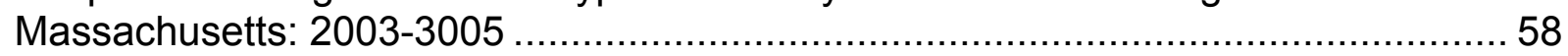

Hospital Discharge Rate for Hypertension by Race/Ethnicity, Boston Region,

Massachusetts: 2003-2005 
Hospital Discharge Rate for Heart Disease by Cities in Boston Region, Massachusetts: 2003-2005 ….................................................................. 59 Heart Disease Mortality Rates by EOHHS Region, Massachusetts: 2003-2005 .......60 Heart Disease Mortality Rates by Cities in Boston Region and Massachusetts: 20032005 60

Heart Disease Death Rates by Race/Ethnicity, Boston Region and Massachusetts : 2003-2005 61

Prevalence of Diabetes, Massachusetts: 1994-2005

Diabetes Hospital Discharges by EOHHS Region, Massachusetts: 2003-2005........ 62

Diabetes Hospital Discharges by Cities in Boston Region and Massachusetts: 2003-

2005 62

Diabetes Hospital Discharges by Race/Ethnicity, Boston Region and Massachusetts: 2003-2005. 63

Diabetes Mortality Rates by EOHHS Region and Massachusetts: 2003-2005......... 63 Diabetes Mortality Rate by Cities in Boston Region and Massachusetts: 2003-200564 Diabetes Mortality Rates by Race/Ethnicity Boston Region and Massachusetts: 20032005 64 Summary Indicators for Overweight/Obesity, by EOHHS Regions, Massachusetts : 2005 65

Summary Indicators for Chronic Disease Outcomes for Overweight/Obesity by EOHHS Regions

CANCER 67

Adults Ages 50+ who have had a Sigmoidoscopy or Colonoscopy in Past 5 Years by EOHHS Regions, Massachusetts: 2005. 69 Incidence Rates for Invasive Colorectal Cancer by EOHHS Region, Massachusetts: 1990-2003. 69

Incidence Rates for Early Stage Colorectal Cancer, Males, by EOHHS Region,

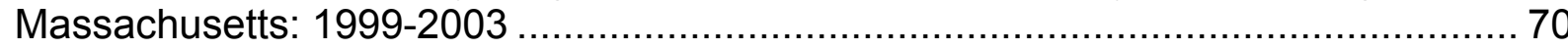
Incidence Rates for Early Stage Colorectal Cancer, Females, by EOHHS Region, Massachusetts: 1999-2003 …............................................................. 70 Colorectal Cancer Mortality Rates by Cities in Boston Region and Massachusetts: 2003-2005 71 Men Ages 50+ who had a Prostate-Specific Antigen Test in the Past 2 years by EOHHS Region, Massachusetts: 2004 71 Men Ages 50+ who had a Digital Rectal Exam in the Past Two Years by EOHHS Region, Massachusetts: 2004

Adults Ages $50+$ who had a Blood Stool Test in the Past Two Years by EOHHS Region, Massachusetts: 2005 72 Incidence Rates for Invasive Prostate Cancer by EOHHS Region, Massachusetts: 1999- 2003

Prostate Cancer Incidence Rates by Cities in Boston Region, Massachusetts: 1999-

2003 73 Incidence Rates for Early Stage Prostate Cancer by EOHHS Region, Massachusetts: 1999-2003 74

Prostate Cancer Mortality Rates by EOHHS Region, Massachusetts: 2005 74 
Women who had a Clinical Breast Exam in the Past Two Years by Race and Ethnicity, Boston Region and Massachusetts: 2004 .......................................... 75 Women Ages 40+ who had a Clinical Breast Exam in the Past Two Years by EOHHS

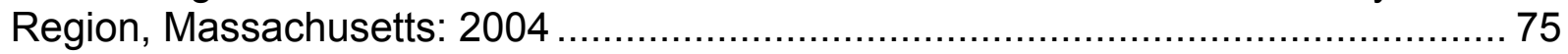
Women Ages 40+ who have had a Mammography in Past 2 Years by EOHHS

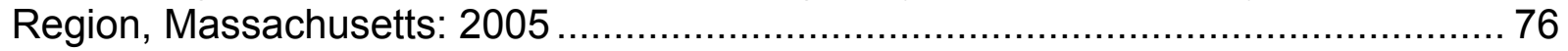
Incidence Rates for Early Stage Female Breast Cancer by EOHHS Region, Massachusetts : 1999-2003 ........................................................................ 76 Incidence Rates for Invasive Female Breast Cancer by EOHHS Region, Massachusetts: 1999-2003 …................................................................. 77 Female Breast Cancer Mortality Rates by Race and Ethnicity, Boston Region,

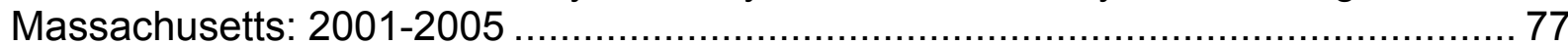
Smoking During Pregnancy, Boston and Massachusetts: 1990-2005.................... 78 Smoking During Pregnancy by EOHHS Regions, Massachusetts: 2005 ................ 78 Percent of Mothers Smoking During Pregnancy for Cities in Boston Region: 2005 .. 79 Smoking During Pregnancy by Race and Ethnicity, Boston Region and Massachusetts: 2005 ......................................................................... 79 Incidence Rates for Invasive Lung Cancer by EOHHS Region, Massachusetts: 1999-

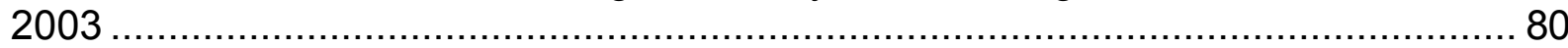
Incidence Rates for Early Stage Lung Cancer, Males, by EOHHS Region,

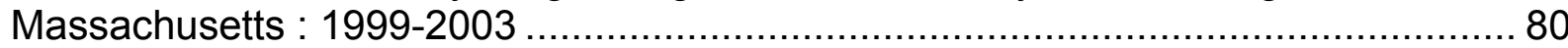
Incidence Rates for Early Stage Lung Cancer, Females, by EOHHS Region,

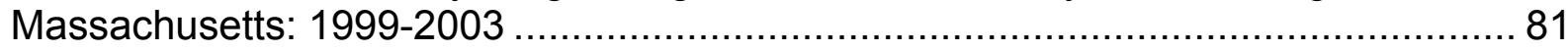
Lung Cancer Mortality Rates by EOHHS Region, Massachusetts: 2005 ................. 81 Lung Cancer Mortality Rates by Cities in Boston Region and Massachusetts: 2003-

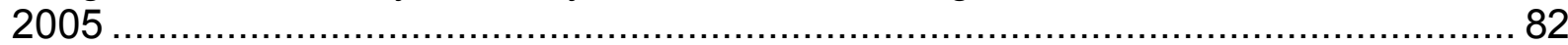

Lung Cancer Mortality Rates by Race and Ethnicity, Boston Region and

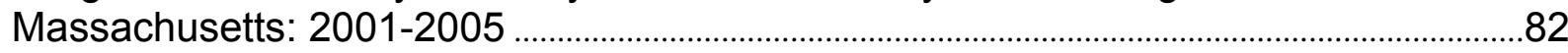
Adults who Currently Smoke by EOHHS Regions, Massachusetts: $2005 \ldots \ldots \ldots \ldots \ldots . .83$ Current Smoker by Race and Ethnicity, Boston Region and Massachusetts: 2005 .. 83 Summary Indicators for Cancer Screening by EOHHS Regions, Massachusetts:

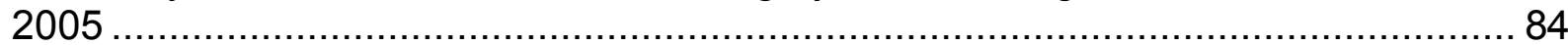
Summary Cancer Indicators (Early Stage) by EOHHS Regions, Massachusetts:

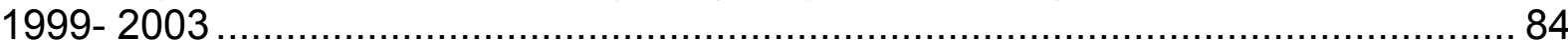
Summary Cancer Indicators (Late Stage) by EOHHS Regions, Massachusetts: 19992003 85 Summary Invasive Cancer Incidence Rates by EOHHS Regions, Massachusetts:

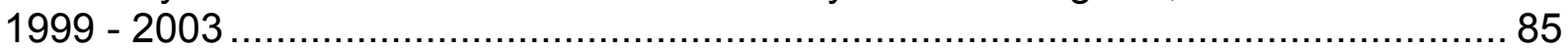
Summary Cancer Mortality Indicators by EOHHS Regions, Massachusetts: 2005 .....86

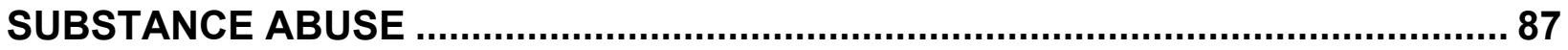

Alcohol Use in Past Month, Persons Aged 12 to 20 Years, by EOHHS Region, Massachusetts : 2002-2004 ........................................................................ 89 Binge Drinking in Past Month, Persons Aged 12 to 20 Years, by EOHHS Region, Massachusetts : 2002-2004 
Any Illicit Drug Use in Past Month, Persons Aged 12+ Years, by EOHHS Region, Massachusetts: 2002-2004

Any Illicit Drug Use Other Than Marijuana use in Past Month, Persons Aged 12+ Years, by EOHHS Region, Massachusetts: 2002-2004 …................................ 90 Alcohol Dependence or Abuse in Past Year, Persons Aged 12+ Years, by EOHHS Region, Massachusetts : 2002-2004

Dependence or Abuse of Any Illicit Drug or Alcohol in Past Year, Persons Aged 12+ Years by EOHHS Region, Massachusetts : 2002-2004 ..................................... 91 Adults who Binge Drink, by EOHHS Region, Massachusetts : 2005 ...................... 92 Adults who Used Illicit Drugs in Past Month by EOHHS Region, Massachusetts :

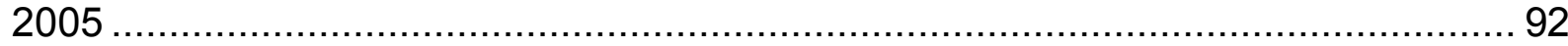

Substance Abuse Treatment Program Admissions by EOHHS Region, Massachusetts : 2005 93 Substance Abuse Treatment Program Admissions, Alcohol Primary Substance, by EOHHS Region, Massachusetts : 2005

Abuse Treatment Program Admissions, Alcohol Primary Substance, Cities in Boston Region and Massachusetts: 2005

Substance Abuse Treatment Program Admissions, Cocaine Primary Substance, by EOHHS Region, Massachusetts: 2005 94 Substance Abuse Treatment Program Admissions, Cocaine Primary Substance, Cities in Boston Region and Massachusetts: 2005 95 Substance Abuse Treatment Program Admissions, Heroin Primary Substance, by EOHHS Region, Massachusetts: 2005 Substance Abuse Treatment Program Admissions, Heroin Primary Substance, Cities in Boston Region and Massachusetts: 2005 96 Opioid-related Emergency Visit Rates by EOHHS Region, Massachusetts: 20032005 96

Opioid-related Emergency Department Visit Rate by Boston Cities and Massachusetts : 2005 97 Opioid-related Emergency Department Visit Rates by Race and Ethnicity Boston Region, Massachusetts : 2003-2005 ........................................................ 97 Massachusetts City and Town FY03-05, Total Number of Opioid Overdose Related Deaths.

Massachusetts City and Town FY03-05, Total Number of Opioid Overdose Related

Deaths 98

Opioid-Related Fatal Overdoses by EOHHS Region, Massachusetts : 2003-2005 .. 99 Opioid-Related Fatal Overdoses by Cities in Boston Region and Massachusetts : 2003-2005

Opioid-Related Fatal Overdoses by Race/Ethnicity, Boston Region and

Massachusetts : 2003-2005

Massachusetts City and Town FY03-05, Total Number of Poly-Drug Overdose related Deaths 100 Massachusetts City and Town FY03-05, Total Number of Poly-Drug Overdose related Deaths 101

Summary Substance Abuse Indicators Percentages by EOHHS Regions ............. 101 Summary Substance Abuse Indicators by EOHHS Region, Massachusetts: 2005. 102 
Summary Opioid Indicators by EOHHS Regions, Massachusetts: 2003-2005....... 102

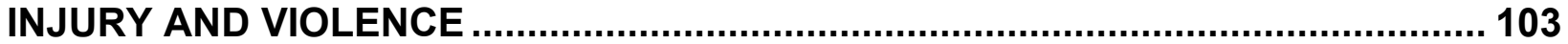

Causes of Injury Deaths, Boston Region: 2005 ............................................ 105

Emergency Department Visit Rates for Injuries and Poisonings by EOHHS Region,

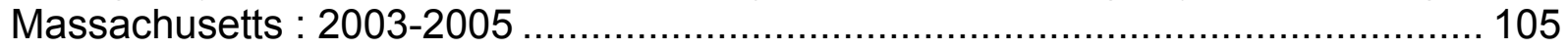
Injury-Related Hospital Discharge Rates by EOHHS Region and Massachusetts:

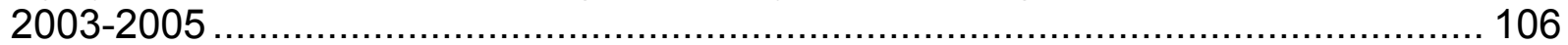
Injury-Related Hospital Discharge Rates by Race and Ethnicity, Boston Region and Massachusetts: 2003-2005 ….............................................................. 106 Injury Death Rates by EOHHS Region and Massachusetts: 2003-2005 ................ 107 Emergency Department Visit Rates for Injuries \& Poisonings by Race/Ethnicity, Boston Region and Massachusetts : 2003-2005 .......................................... 107 Hospital Discharge Rates for Assaults, by EOHHS Region, Massachusetts: 2003-

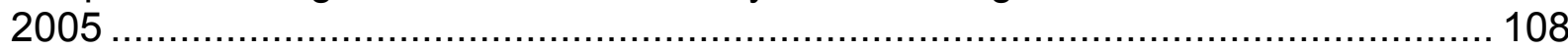
Hospital Discharge Rates for Assaults by Race/Ethnicity, Boston Region and Massachusetts: 2003-2005 ................................................................. 108 Homicides by EOHHS Region, Massachusetts : 2003-2005 .............................. 109 Homicides in Boston Region, Massachusetts : 2003-2005 ................................ 109 Homicide Rates by Race/Ethnicity, Boston Region and Massachusetts: 2003-2005110 Hospital Discharge Rates for Self-Inflicted Injury, by EOHHS Region, Massachusetts : 2003-2005 ................................................................... 110 Hospital Discharge Rates for Self-Inflicted Injuries by Race/Ethnicity, Boston Region

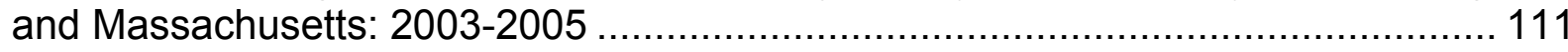
Suicide by EOHHS Region, Massachusetts: 2003-2005 ............................... 111 Suicide Rates by Race/Ethnicity, Boston Region and Massachusetts: 2003-2005 . 112 Hospital Discharge Rates for Motor Vehicle-Related Injuries by EOHHS Region and Massachusetts : 2003-2005 .................................................................... 112 Hospital Discharge Rates for Motor Vehicle-Related Injuries by Race/Ethnicity, Boston Region and Massachusetts: 2003-2005 ............................................. 113 Emergency Department Visit Rates for Motor Vehicle-Related Injuries by Boston Cities and Massachusetts : 2003-2005 …............................................... 113 Motor Vehicle-Related Mortality Rates by EOHHS Region, Massachusetts : 2003-

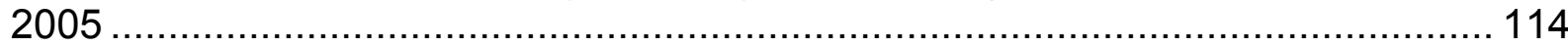
Motor Vehicle-Related Death Rates by Race/Ethnicity, Boston Region and Massachusetts: 2003-2005 .......................................................................... 114 Emergency Department Visit Rates for Traumatic Brain Injury, by EOHHS Region,

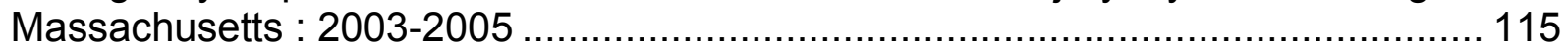
Emergency Department Visit Rates for Traumatic Brain Injury by Race/Ethnicity, Boston Region and Massachusetts : 2003-2005 ............................................ 115 Emergency Department Visit Rates for Traumatic Brain Injury, Cities in Boston Region and Massachusetts : 2003-2005 ..................................................... 116 Hospital Discharge Rates for Traumatic Brain Injuries by EOHHS Region, Massachusetts : 2003-2005 116 Hospital Discharge Rates for Traumatic Brain Injury by Race/Ethnicity, Boston Region and Massachusetts : 2003-2005. 
Emergency Department Visit Rates for Firearm Injuries, by EOHHS Region, Massachusetts: 2003-2005

Emergency Department Visit Rates for Firearm Injuries, Boston Region and Massachusetts: 2003-2005

Emergency Department Visit Rates for Firearm Injuries by Race/Ethnicity, Boston

Region and Massachusetts: 2003-2005

Firearm Death Rates by EOHHS Region, Massachusetts: 2003-2005 ................. 119

Firearm Death Rates by Boston Region, Massachusetts: 2003-2005 ................... 119

Firearm Death Rates by Race/Ethnicity, Boston Region and Massachusetts: 2003-

2005 120

Hospitalization Rates for Fall-Related Injuries Ages 65+, by EOHHS Region,

Massachusetts : 2003-2005

Fall-Related Injury Death Rates Persons 65+ by EOHHS Region, Massachusetts :

2003-2005

Hospital Discharge Rates for Fall-Related Injuries by EOHHS Region and

Massachusetts : 2003-2005

Hospital Discharge Rates for Fall-Related Injuries by Race/Ethnicity, Boston Region

and Massachusetts : 2003-2005

Emergency Department Visit Rates for Fall-Related Injuries, by EOHHS Region,

Massachusetts : 2003-2005

Emergency Department Visit Rates for Fall-Related Injuries by Race/Ethnicity,

Boston Region and Massachusetts : 2003-2005.

Fall-Related Injury Mortality Rates by EOHHS Region, Massachusetts : 2003-2005123

Fall-Related Injury Mortality Rates by Race/Ethnicity, Boston Region and

Massachusetts : 2003-2005 124

Work-related Injuries, Emergency Department Visits per 100 Full Time Equivalents, Massachusetts Counties: 2004-2005 ............................................................ 124 Work-related Injuries among Teens, Emergency Department Visits per 100 Full Time Equivalents, Massachusetts Counties: 2004-2005....................................... 125 Work-related Burn Injuries, Emergency Department Visits per 10,000 Full Time Equivalents, Massachusetts Counties: 2004-2005.......................................... 125 Work-related Amputations, Emergency Department Visits per 10,000 Full Time Equivalents, Massachusetts: 2004-2005. 126 Work-related Injuries, Hospitalizations per 10,000 Full Time Equivalents, Massachusetts Counties: 2004-2005 126

Number and Rate of Fatal Occupational Injuries by year, Massachusetts: 19912005

Summary Injury/Violence Hospitalization Indicators by EOHHS Regions,

Massachusetts : 2003-2005

Summary Injury/Violence Emergency Department Indicators by EOHHS Regions,

Massachusetts : 2003-2005 128 Summary Injury/Violence Mortality Indicators by EOHHS Regions, Massachusetts : 2003-2005 128

HIVIAIDS 
HIV Incidence, by EOHHS Region, Massachusetts: 2005 ............................... 131

HIV Incidence, by Cities in Boston Region and Massachusetts: 2005 .................. 132

Persons Living with HIV, Cities in Boston Region, Massachusetts: 2005............... 132

AIDS Incidence, by EOHHS Region, Massachusetts: 2005 .............................. 133

Persons Living with AIDS, by EOHHS Region, Massachusetts : 2005 ................. 133

HIVIAIDS Mortality Rates, by EOHHS Region, Massachusetts : 2003-2005 …...... 134

HIVIAIDS Mortality Rates in Boston Region and Massachusetts : 2003-2005 ....... 134

HIVIAIDS Death Rates by Race/Ethnicity, Boston Regions and Massachusetts: 2003

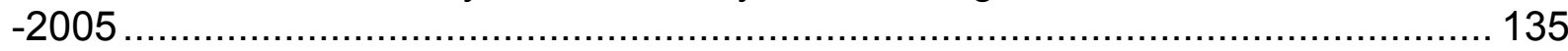

Summary HIVIAIDS Indicators by EOHHS Regions ....................................... 135

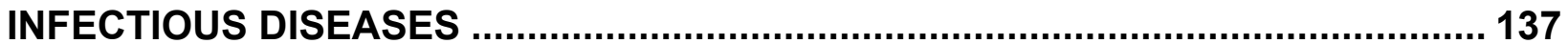

Chlamydia Incidence, Boston Region and Massachusetts : 1989-2005 ................. 139

Chlamydia Incidence by EOHHS Region, Massachusetts: 2005 ......................... 139

Chlamydia Incidence Rate by Emergency Preparation Region, 2006 ................... 140

Gonorrhea Incidence by EOHHS Region, Massachusetts : 2005 ....................... 140

Syphilis Incidence, Boston Region and Massachusetts : 1989-2005 .................... 141

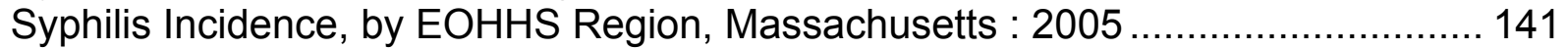

Syphilis Incidence, Metrowest Cities and Massachusetts : 2003-2005 .................. 142

Tuberculosis Incidence, by EOHHS Region, Massachusetts : 2004 ..................... 142

Tuberculosis Incidence, by EOHHS Region, Massachusetts : 2006 ...................... 143

Confirmed Tuberculosis Cases by Race/Ethnicity and EOHHS Region,

Massachusetts: 2006

Percent Confirmed Tuberculosis Cases by EOHHS Region, Massachusetts : 2006144

Percent of Confirmed Tuberculosis Cases by Race/Ethnicity and EOHHS Region,

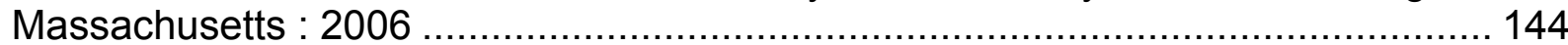

Tuberculosis Cases by EOHHS Region, Massachusetts : 1993-2006 .................. 145

Percent of Confirmed Tuberculosis Cases by Race/Ethnicity and EOHHS Region,

Massachusetts : 2006 .................................................................... 145

Hepatitis C Average Incidence Rates, by EOHHS Region, Massachusetts: 1992-

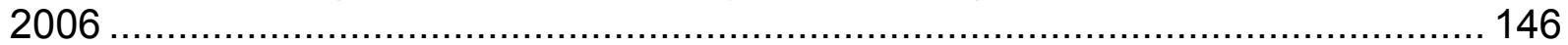

Massachusetts Hepatitis C Infection Average per Year, by Town, 1992-2006 ........ 146

Hepatitis C Incidence Rates, by EOHHS Region, Massachusetts : 2006 ............... 147

Massachusetts Hepatitis C Infection Average per Year, by Town, 2006 ................ 147

Lyme Disease Incidence by EOHHS Region, and Massachusetts: 2004 ............... 148

Massachusetts Lyme Disease Case Rates, by Town, 2005 ............................... 148

Pneumonia and Influenza Hospital Discharges by EOHHS Region, Massachusetts:

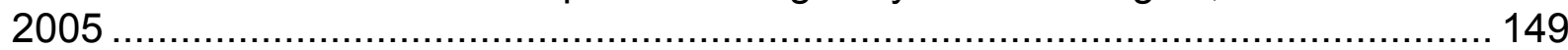

Hospital Discharges Rates for Pneumonia and Influenza by Cities in Boston Region

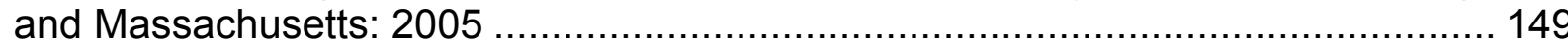

Summary Infectious Disease Indicators, by EOHHS Regions............................. 150

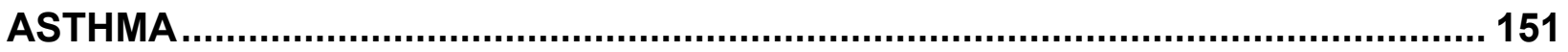

Asthma Prevalence for School Children, Grades K-8, by CHNA within EOHHS

Region, Massachusetts : 2004-2005 
Pediatric Asthma Prevalence Among School Children, Grades K-8, by EOHHS Region, Massachusetts : 2005-2006 .......................................................... 153

Asthma Hospital Discharges by EOHHS Region, Massachusetts: 2005............... 154 Asthma Emergency Department Visits, by EOHHS Region, Massachusetts: 2005154 Emergency Department Visit Rates for Asthma by Race/Ethnicity, Boston Region and

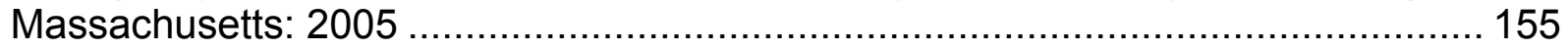

Adults who Currently have Asthma by EOHHS Region, Massachusetts: 2005 ....... 155 Asthma ER Visits, Children Ages 0-4, by EOHHS Region, Massachusetts : 2005 . 156 Asthma ER Visits, Children Ages 5-9, by EOHHS Region, Massachusetts : 2005 . 156 Asthma ER Visits, Children Ages 10-14, by EOHHS Region, Massachusetts :

2005

Asthma ER Visits, Children Ages 0-14, by EOHHS Region, Massachusetts : 2005157 Asthma Emergency Department Visit Rates, Children Ages 0-14, Boston Region and Massachusetts : 2005 158 Asthma Emergency Department Visit Rates by Race and Ethnicity, Children Ages 014, Boston Region and Massachusetts : 2005 158 Pediatric Asthma Prevalence Among School Children, Grades K-8, EOHHS Region and Massachusetts : 2005 159

Pediatric Asthma Emergency Visit Rates by Towns Among Children, Ages <5,

Boston Region and Massachusetts : 2004-2005 159 Pediatric Asthma Hospitalization Rates by Towns Among Children, Ages < , Boston Region and Massachusetts : 2004-2005 ................................................. 160 Summary Asthma Indicators, by EOHHS Regions, Massachusetts : 2005 ............. 160 Summary Asthma Indicators, by EOHHS Regions, Massachusetts : 2005 ............. 161

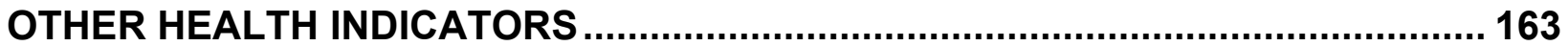

Total Cases of Elevated Blood Lead Levels ( $>=15 \mu \mathrm{G} / \mathrm{DL}$ ), by EOHHS Region,

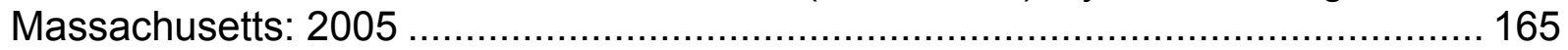
Lead Poisoning Prevalence Rates (BLL $\geq 25 \mu \mathrm{G} / \mathrm{DL}$ ), Children 9-48 Months, by EOHHS Region, Massachusetts: 2006 .............................................................. 165 Adults who Report Experiencing Intimate Partner Violence by Gender and EOHHS Region, Massachusetts: 2005 ................................................................... 166 Adults who Report Having a Disability by EOHHS Region, Massachusetts : 2005 . 166 Adults who Report Having a Disability and needing help with activities, by EOHHS

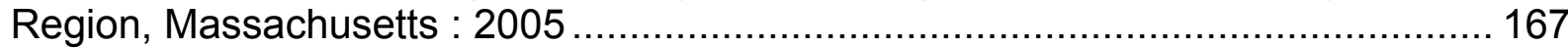
Adults with Fair or Poor Health, by EOHHS Region, Massachusetts : 2005 ........... 167 Adults who Reported Fair or Poor Health by Race and Ethnicity, Boston Region and Massachusetts : 2005 ................................................................................. 168 Adults who Currently Report Fair or Poor Health by Race and Ethnicity, Boston Region and Massachusetts : 1999-2005 ....................................................... 168 Adults with Poor Mental Health for 15+ Days in Past Month, by EOHHS Region,

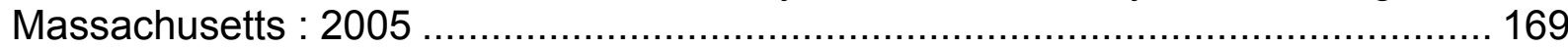
Summary Other Health Indicators, by EOHHS Regions...................................... 169 Adults who Visited a Dentist in Past Year, by EOHHS Region, Massachusetts : 2004 
Adults who have lost 6 or More Teeth to Decay or Disease, by EOHHS Region, Massachusetts : 2004 170

Refugees Arrivals: Boston Region, 2002-2006 .......................................... 171

Refugees Health Assessment Findings: Boston Region, 2002-2006 ................... 171

Summary, Refugees by EOHHS Regions, Massachusetts: 2002-2006 …............. 172

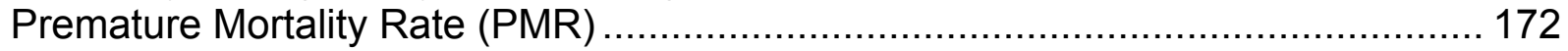

Premature Mortality Rates (PMR) by EOHHS Region, Massachusetts : 2005........ 173

PMR - Boston Region by City/Town, Massachusetts : 2005 ............................. 173

Median Household Income and PMR by EOHHS Region, Massachusetts: 2005 ... 174

Premature Mortality Rate (PMR) by EOHHS Regions: 2005 .............................. 174

SUMMARY

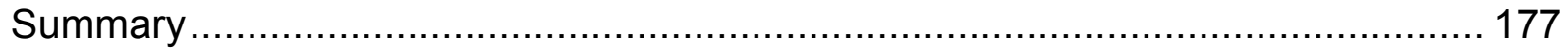

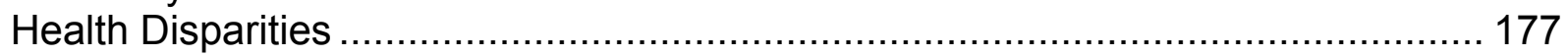

APPENDIX

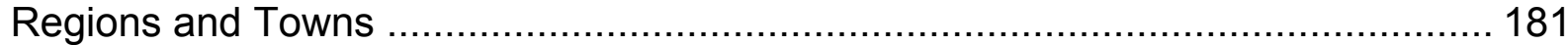

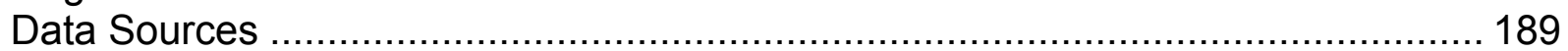




\section{NOTE TO READERS}

\section{Background}

This report presents important health indicators for your region, which were developed as background material for the Regional Health Dialogues being held across the Commonwealth in May and June 2007. These dialogues offer an opportunity for you to have a conversation with the Commissioner of Public Health, learn the latest health information for your region, identify your resources and areas of need, and together, with the Massachusetts Department of Public Health, shape a public health partnership.

\section{Contents}

This report provides information about the socio-demographics, health care access, births, deaths, major chronic and infectious disease rates, substance abuse, and injury and violence for your region. Your region's indicators are compared with those of other regions and to the state as a whole in order to provide you with a sense of the relative health of your region. Many indicators for your region are stratified by race, Hispanic ethnicity, and age in order help determine specific population groups that may require targeted health interventions. We have also provided information about the health status of one or more large communities in your region, which may be influencing your regional outcomes. We hope that this collection of important and diverse health indicators will stimulate discussions of local health priorities, as well as serving as a resource for health information and a reference for data that are available from the Massachusetts Department of Public Health. Please contact us with your comments and suggestions. 
INTRODUCTION 


\section{Goals for holding $\underline{\text { Regional Dialogues }}$}

- Introduce new DPH leadership

- Present latest health data on your particular region

- Talk with residents and providers about data and identify areas of need

- Gather feedback to inform process of setting DPH priorities

\section{Goals of New DPH leadership}

- Use data and community input to set new priorities \& reshape the Department

- Rely on science and evidence-based process to guide the work

- Strengthen ties with and support for local health and local agencies

- Increase resources to and visibility of public health in Massachusetts 


\section{Timetable}

- Hold 8 regional dialogues with comprehensive data review and identification of key health concerns (by July)

- Identify short list of top priorities by end of August

- Hold second round of community dialogues to discuss action plans/results by end of calendar year 


\section{SOCIODEMOGRAPHIC INDICATORS}




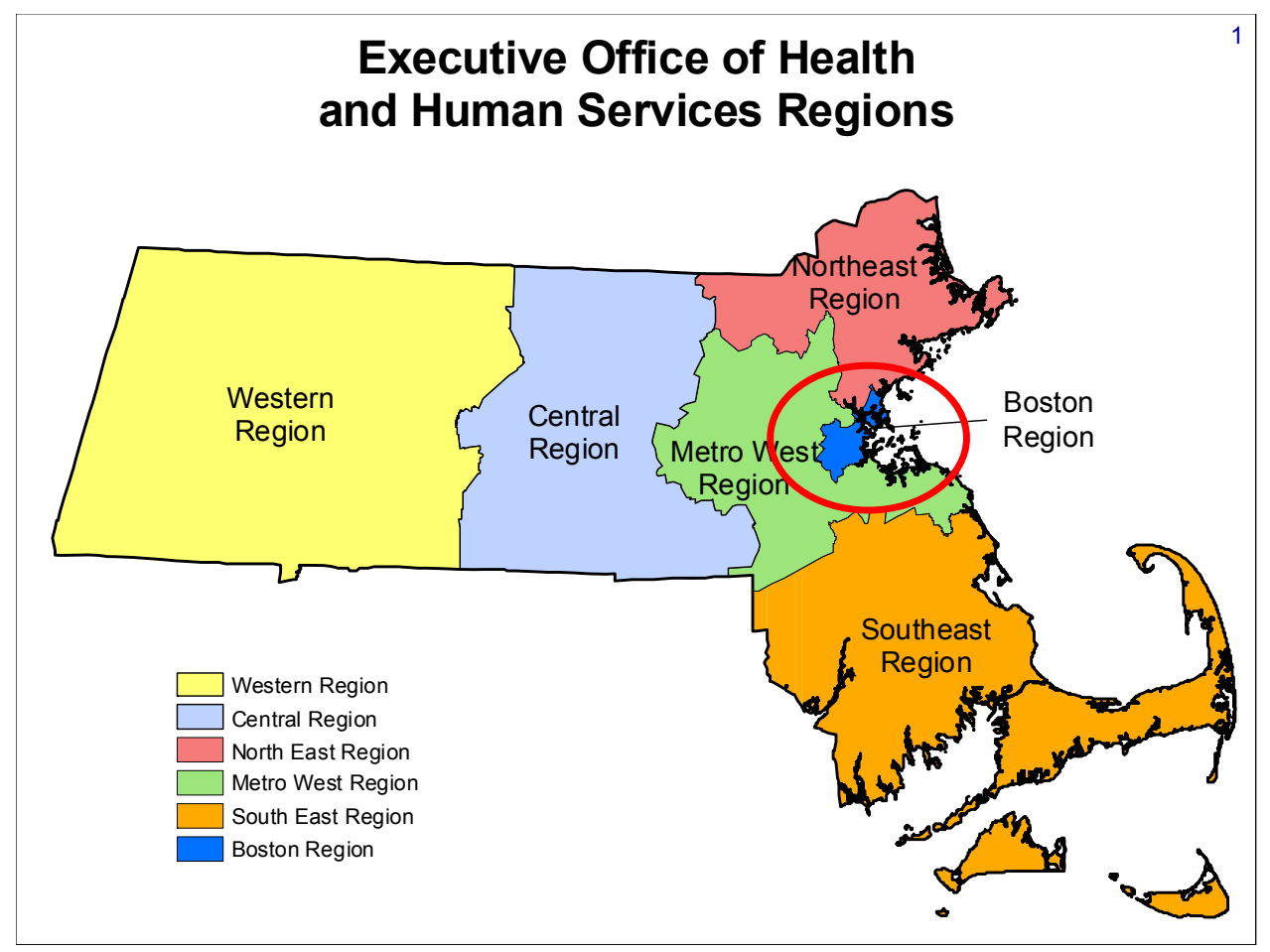

\section{A few comments about the limitations of this presentation}

- A regional analysis has its drawbacks

- Variability within a region - urban vs. rural/suburban (i.e. Boston vs. the rest of the region)

- Variability by sub-population - race matters, poverty matters

- Our data don't reflect all the important health indicators 


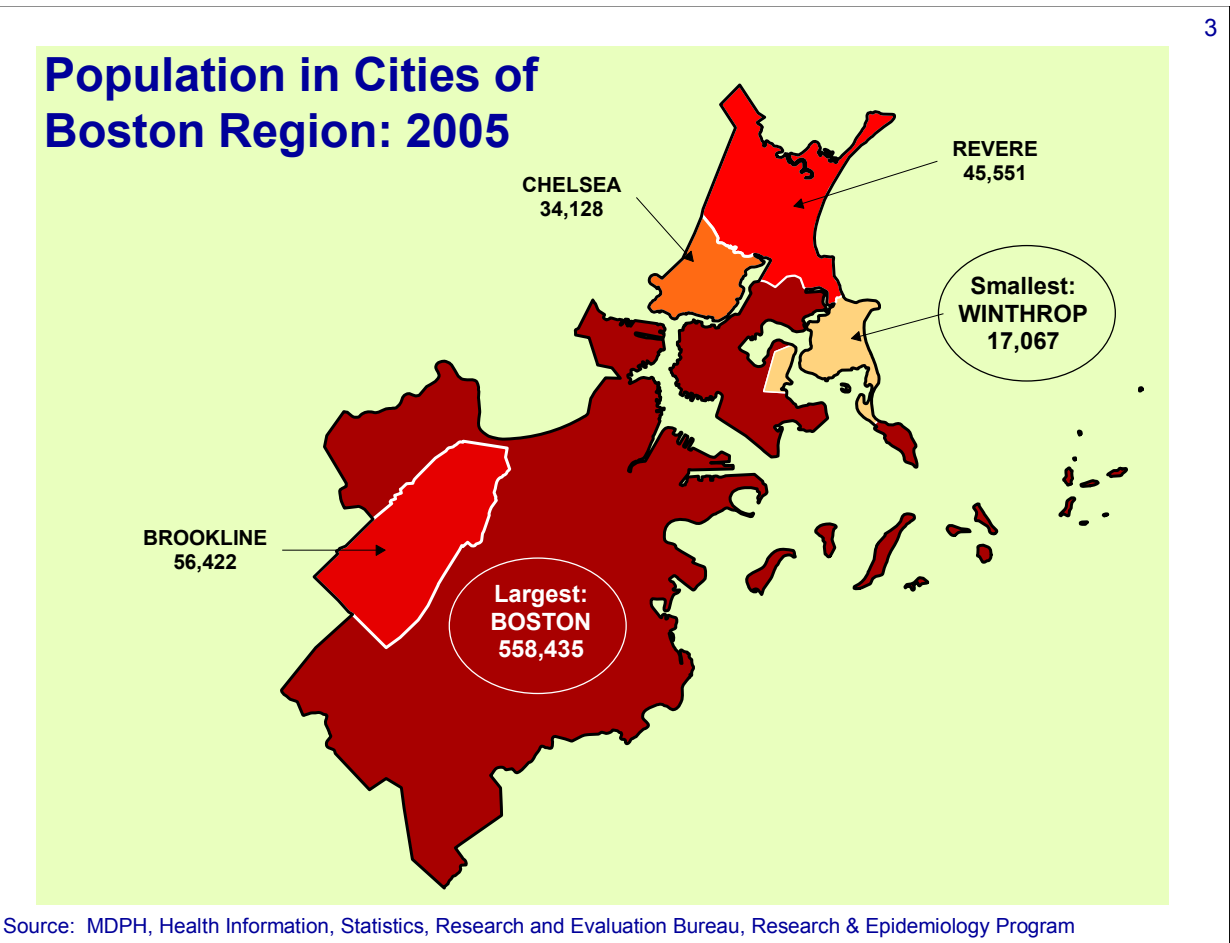

\section{Race and Ethnic Breakdown of Population Boston Region and Massachusetts: 2005}

Boston Region

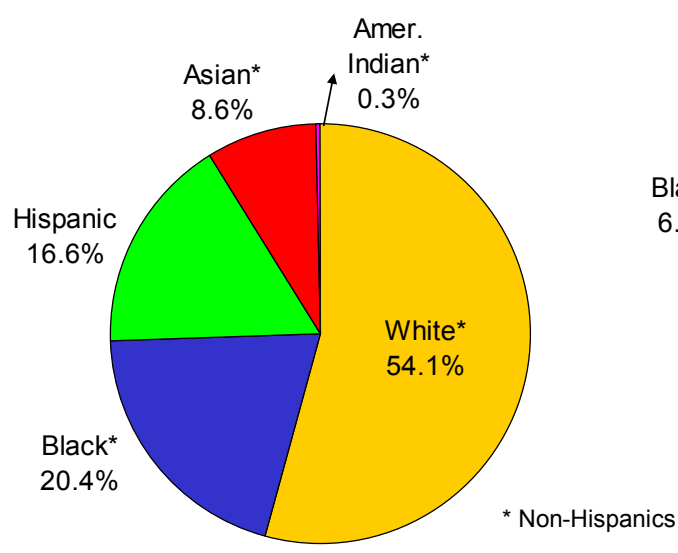

Total Population: 711,603
Massachusetts

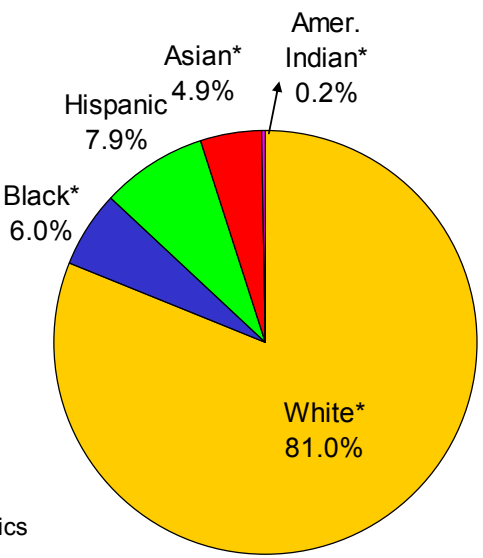

Total Population: $6,436,940$ 
Race and Ethnic Breakdown of Population Cities in Boston Region and Massachusetts: 2005

\begin{tabular}{|c|c|c|c|c|c|c|c|}
\hline $\begin{array}{c}\text { Racial } \\
\text { Hispanic } \\
\text { Group }\end{array}$ & $\begin{array}{c}\text { City of } \\
\text { Boston }\end{array}$ & Brookline & Chelsea & Revere & Winthrop & $\begin{array}{c}\text { Boston } \\
\text { Region }\end{array}$ & $\begin{array}{c}\text { MA } \\
\text { Total }\end{array}$ \\
\hline White* $^{*} 49.9 \%$ & $73 \%$ & $35.9 \%$ & $81.6 \%$ & $93.9 \%$ & $54.1 \%$ & $81 \%$ \\
\hline Black* & $25 \%$ & $4.2 \%$ & $5.6 \%$ & $2.5 \%$ & $1.5 \%$ & $20.4 \%$ & $6 \%$ \\
\hline Hispanic & $16.4 \%$ & $4.8 \%$ & $53.3 \%$ & $10.5 \%$ & $3.1 \%$ & $16.6 \%$ & $7.9 \%$ \\
\hline Asian* & $8.4 \%$ & $17.9 \%$ & $5 \%$ & $5.2 \%$ & $1.3 \%$ & $8.6 \%$ & $4.9 \%$ \\
\hline $\begin{array}{c}\text { American } \\
\text { Indian* }\end{array}$ & $0.3 \%$ & $0.1 \%$ & $0.3 \%$ & $0.2 \%$ & $0.2 \%$ & $0.3 \%$ & $0.2 \%$ \\
\hline
\end{tabular}

* Non-Hispanics

Source: MDPH, Health Information, Statistics, Research \& Evaluation Bureau, Research \& Epidemiology Program

\section{Population by Age Group}

Cities in Boston Region and Massachusetts: 2005

\begin{tabular}{|c|c|c|c|c|c|c|c|}
\hline Ages & $\begin{array}{c}\text { City of } \\
\text { Boston }\end{array}$ & Brookline & Chelsea & Revere & Winthrop & $\begin{array}{c}\text { Boston } \\
\text { Region }\end{array}$ & $\begin{array}{c}\text { MA } \\
\text { Total }\end{array}$ \\
\hline $\begin{array}{c}0-19 \\
\text { years }\end{array}$ & $24 \%$ & $19 \%$ & $30 \%$ & $23 \%$ & $21 \%$ & $23 \%$ & $26 \%$ \\
\hline $\begin{array}{c}20-24 \\
\text { years }\end{array}$ & $11 \%$ & $10 \%$ & $7 \%$ & $5 \%$ & $5 \%$ & $10 \%$ & $7 \%$ \\
\hline $\begin{array}{c}25-44 \\
\text { years }\end{array}$ & $35 \%$ & $34 \%$ & $35 \%$ & $33 \%$ & $34 \%$ & $35 \%$ & $29 \%$ \\
\hline $\begin{array}{c}45-64 \\
\text { years }\end{array}$ & $20 \%$ & $25 \%$ & $18 \%$ & $23 \%$ & $25 \%$ & $20 \%$ & $25 \%$ \\
\hline $\begin{array}{c}65+ \\
\text { years }\end{array}$ & $11 \%$ & $12 \%$ & $11 \%$ & $16 \%$ & $16 \%$ & $11 \%$ & $13 \%$ \\
\hline
\end{tabular}

Source: MDPH, Health Information, Statistics, Research \& Evaluation Bureau, Research \& Epidemiology Program 


\section{Language Spoken at Home, Cities in Boston Region and Massachusetts: 2000}

\begin{tabular}{|c|c|c|c|c|c|c|c|}
\hline $\begin{array}{c}\text { As \% of all } \\
\text { persons ages }>5\end{array}$ & $\begin{array}{l}\text { City of } \\
\text { Boston }\end{array}$ & Brookline & Chelsea & Revere & Winthrop & $\begin{array}{l}\text { Boston } \\
\text { Region }\end{array}$ & $\begin{array}{c}\text { MA } \\
\text { Total }\end{array}$ \\
\hline Only English & $66.6 \%$ & $71 \%$ & $41.6 \%$ & $71 \%$ & $87.1 \%$ & $66.6 \%$ & $81.3 \%$ \\
\hline $\begin{array}{l}\text { Spanish or } \\
\text { Spanish Creole }\end{array}$ & $13.6 \%$ & $3.6 \%$ & $43.8 \%$ & $9 \%$ & $3.6 \%$ & $13.7 \%$ & $6.2 \%$ \\
\hline Chinese & $3.2 \%$ & $5.7 \%$ & $0.6 \%$ & $0.6 \%$ & $0.1 \%$ & $3 \%$ & $1.2 \%$ \\
\hline French Creole & $3.5 \%$ & $0.4 \%$ & $0.8 \%$ & $0.3 \%$ & $0.1 \%$ & $2.8 \%$ & $0.7 \%$ \\
\hline $\begin{array}{l}\text { Portuguese or } \\
\text { Port. Creole }\end{array}$ & $2.3 \%$ & $0.7 \%$ & $2.1 \%$ & $2.4 \%$ & $1.4 \%$ & $2.1 \%$ & $2.7 \%$ \\
\hline Vietnamese & $1.8 \%$ & $0.1 \%$ & $1.9 \%$ & $1 \%$ & $0 \%$ & $1.6 \%$ & $0.5 \%$ \\
\hline Italian & $1.2 \%$ & $0.4 \%$ & $1 \%$ & $6.8 \%$ & $3 \%$ & $1.5 \%$ & $1 \%$ \\
\hline $\begin{array}{l}\text { French (Incl. } \\
\text { Patois, Cajun) }\end{array}$ & $1.4 \%$ & $1.4 \%$ & $0.9 \%$ & $0.6 \%$ & $1 \%$ & $1.3 \%$ & $1.4 \%$ \\
\hline Russian & $1.1 \%$ & $4.4 \%$ & $0.1 \%$ & $0.5 \%$ & $0 \%$ & $1.2 \%$ & $0.6 \%$ \\
\hline $\begin{array}{l}\text { Mon Khmer, } \\
\text { Cambodian }\end{array}$ & $0.1 \%$ & $0.3 \%$ & $0.9 \%$ & $2.5 \%$ & $0.1 \%$ & $0.3 \%$ & $0.4 \%$ \\
\hline Hebrew & $0.1 \%$ & $2.1 \%$ & $0.1 \%$ & $0 \%$ & $0.2 \%$ & $0.3 \%$ & $0.1 \%$ \\
\hline Serbo Croatian & $0.1 \%$ & $0.1 \%$ & $2 \%$ & $0.9 \%$ & $0 \%$ & $0.2 \%$ & $0.1 \%$ \\
\hline
\end{tabular}

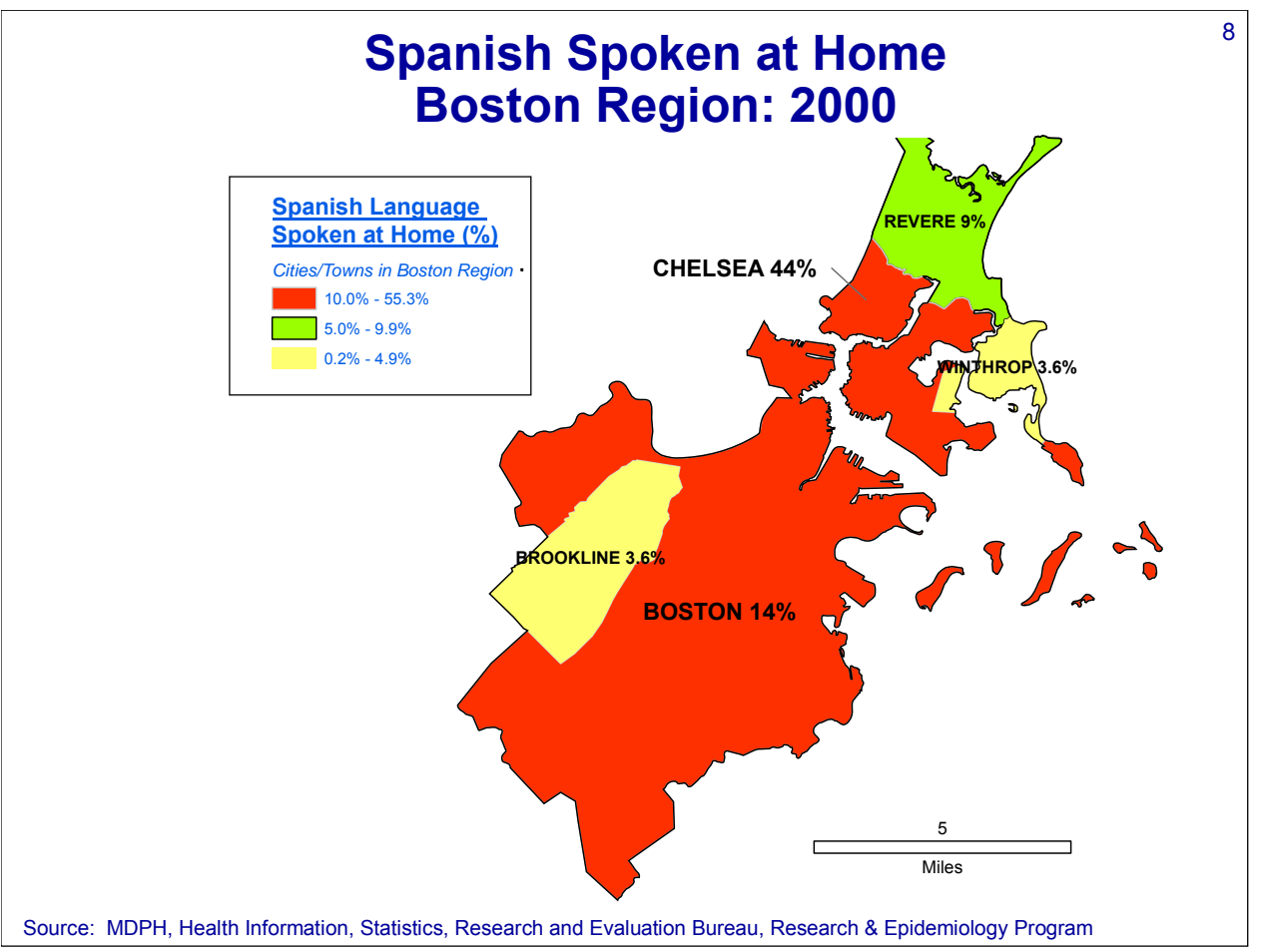




\section{Socio-Demographic Indicators}

Cities in Boston Region and Massachusetts: 2000

\begin{tabular}{|l|c|c|c|c|c|c|c|}
\hline & $\begin{array}{l}\text { City of } \\
\text { Boston }\end{array}$ & Brookline & Chelsea & Revere & Winthrop & $\begin{array}{l}\text { Boston } \\
\text { Region }\end{array}$ & $\begin{array}{c}\text { MA } \\
\text { Total }\end{array}$ \\
\hline $\begin{array}{l}\text { Per Capita } \\
\text { Income }\end{array}$ & $\$ 23,353$ & $\$ 44,327$ & $\$ 14,628$ & $\$ 19,698$ & $\$ 27,374$ & $\$ 24,413$ & $\$ 25,952$ \\
\hline $\begin{array}{l}\text { Those living } \\
\text { below 100\% } \\
\text { poverty }\end{array}$ & $19.5 \%$ & $9.3 \%$ & $23.3 \%$ & $14.6 \%$ & $5.5 \%$ & $18.2 \%$ & $9.3 \%$ \\
\hline $\begin{array}{l}\text { Less than a } \\
\text { High School } \\
\text { Education }\end{array}$ & $21.1 \%$ & $3.7 \%$ & $40.5 \%$ & $23.3 \%$ & $10 \%$ & $20.3 \%$ & $15.2 \%$ \\
\hline $\begin{array}{l}\text { Speak } \\
\text { language other } \\
\text { than English at } \\
\text { home }\end{array}$ & $33.4 \%$ & $29.0 \%$ & $58.4 \%$ & $29.1 \%$ & $12.9 \%$ & $21.9 \%$ & $18.7 \%$ \\
\hline \begin{tabular}{l} 
Foreign born \\
\hline $\begin{array}{l}\text { Unemployed }\end{array}$
\end{tabular} & $25.8 \%$ & $26.6 \%$ & $36.1 \%$ & $21 \%$ & $8.7 \%$ & $25 \%$ & $12.2 \%$ \\
\hline
\end{tabular}

\section{Highest and Lowest Per Capita Income Communities ${ }^{1}$ in Boston Region: 2000}

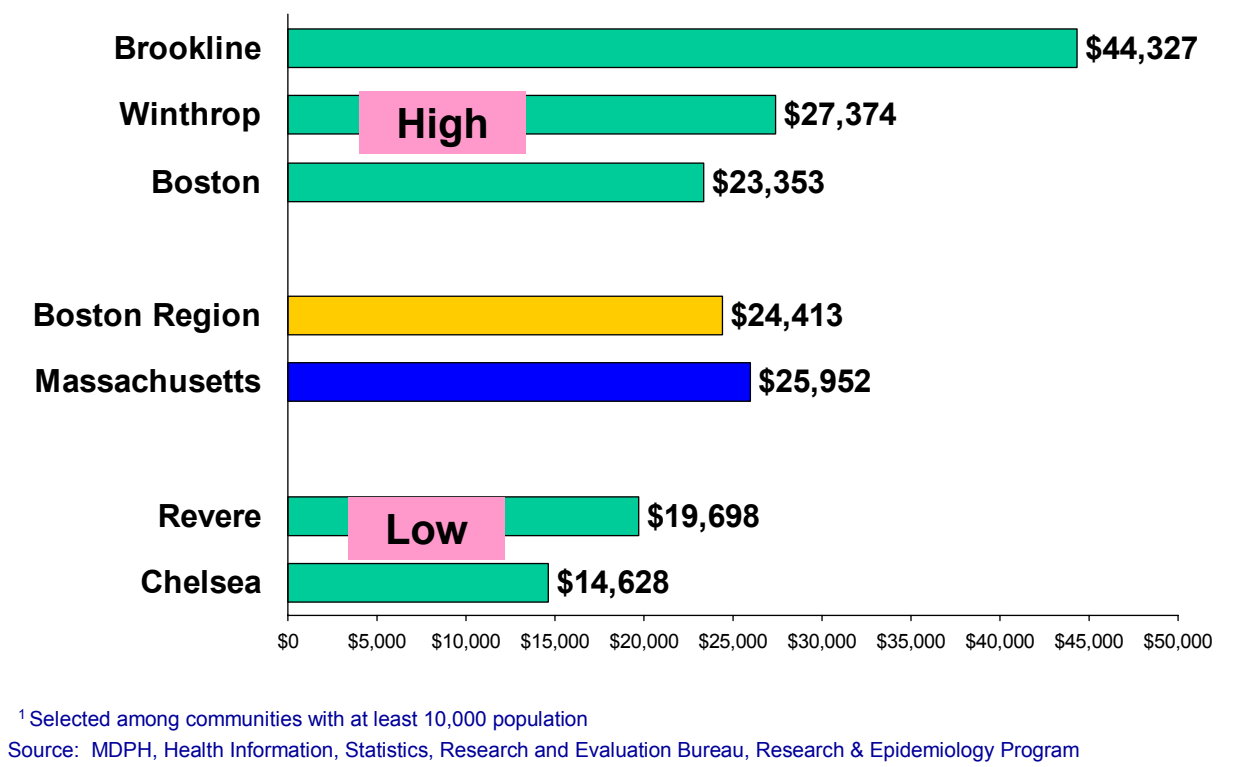


HEALTH CARE ACCESS 
Adults who Have No Health Insurance by EOHHS Region, Massachusetts: 2005
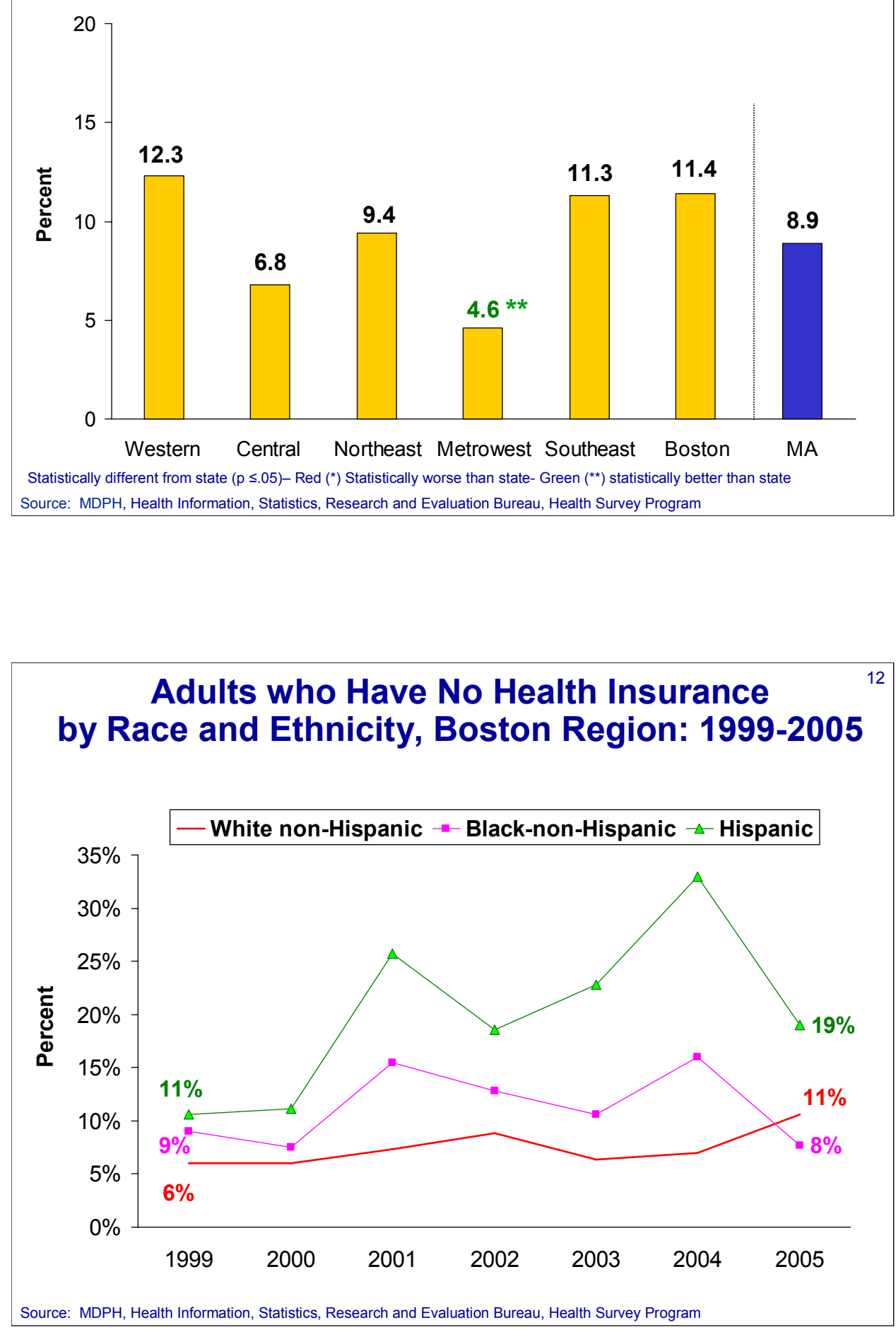


\section{Health Insurance Access \\ Boston Region and Massachusetts: 2005}

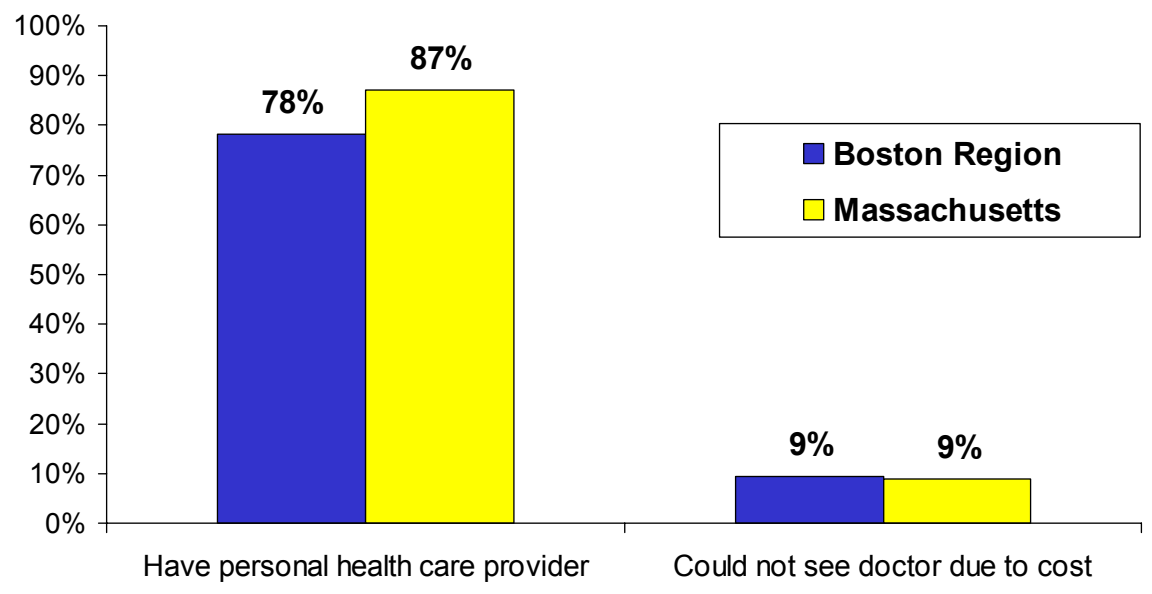

* Statistically Significantly different from state $(p \leq .05)$ - Red: Statistically worse than state- green: statistically better than state Source: MDPH, Health Information, Statistics, Research and Evaluation Bureau, Health Survey Program

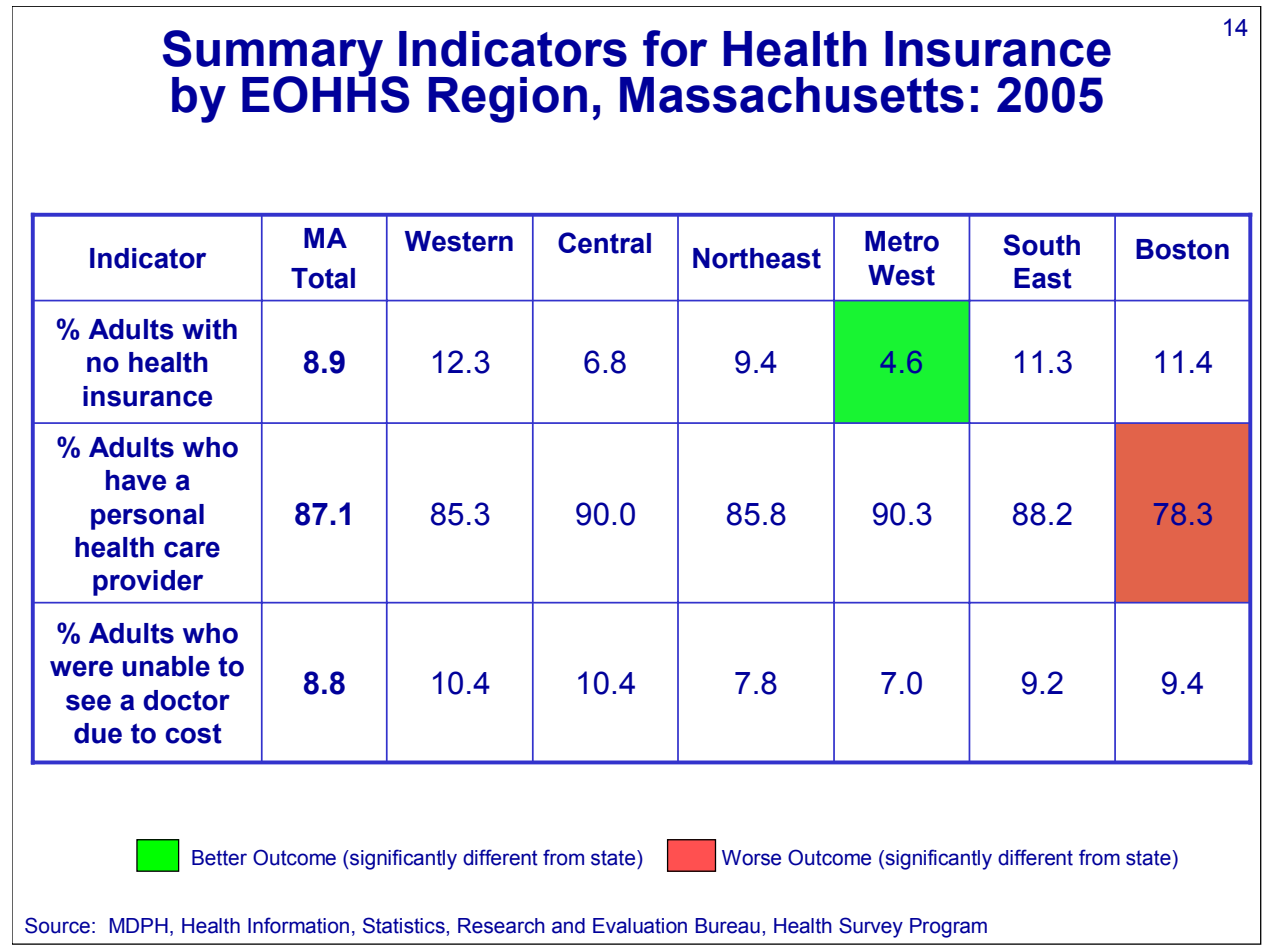




\section{BIRTH INDICATORS}



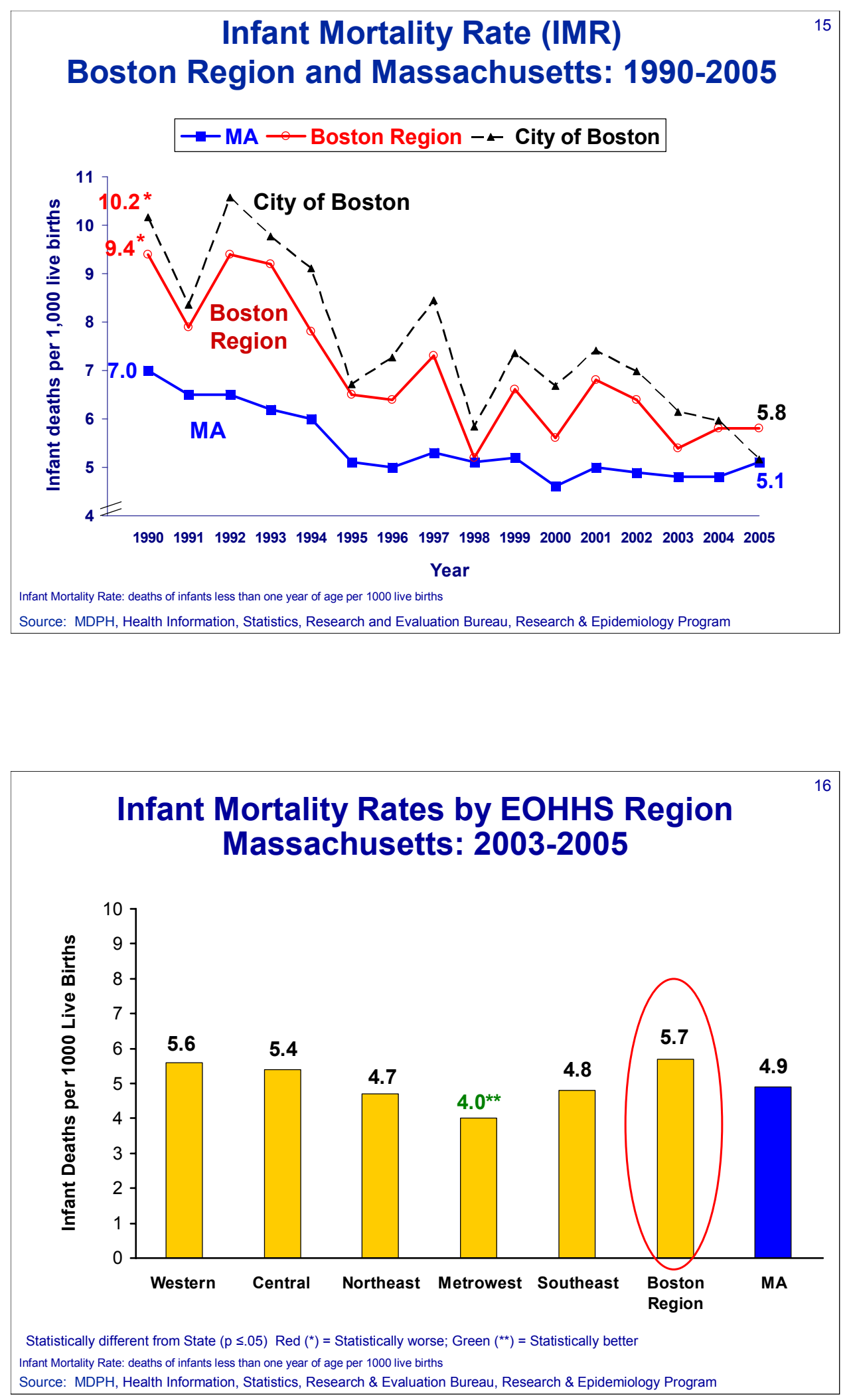
Infant Mortality Rates by Race/Ethnicity Boston Region and Massachusetts: 2003-2005

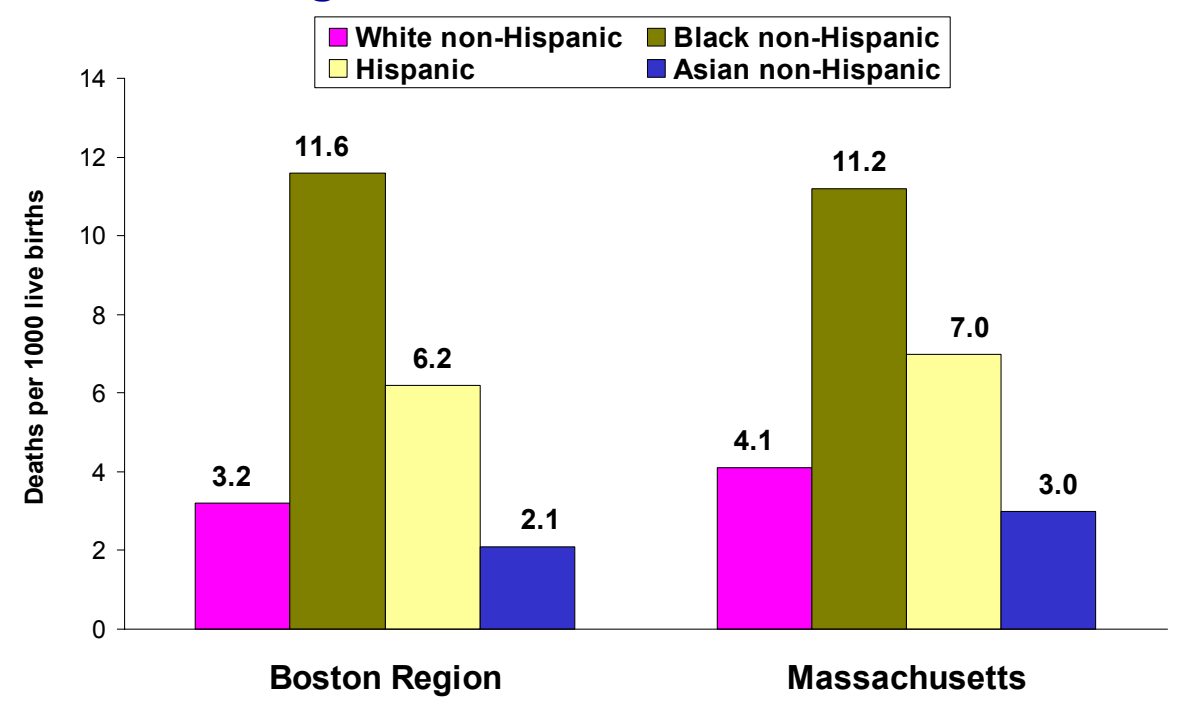

Statistically different from state $(p \leq .05)-\operatorname{Red}\left({ }^{*}\right)$ Statistically worse than state- Green $\left({ }^{* *}\right)$ statistically better than state Source: MDPH, Health Information, Statistics, Research and Evaluation Bureau, Research \& Epidemiology Program

\section{Low Birthweight Boston Region and Massachusetts: 1990-2005}

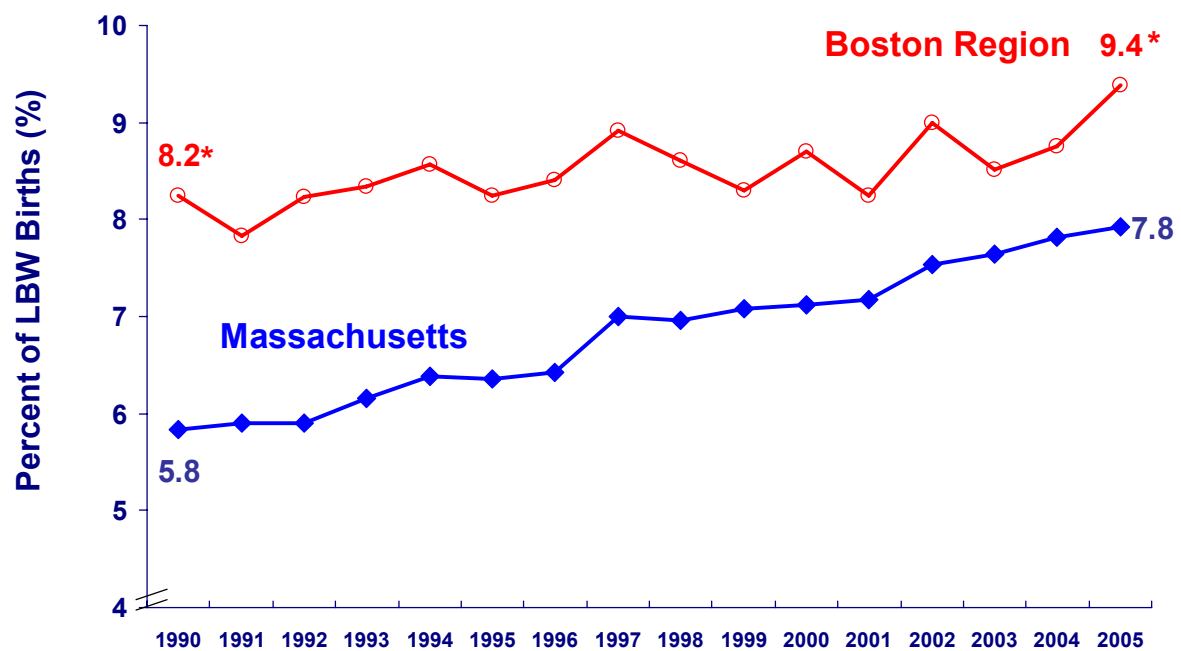


Low Birthweight - Cities in Boston Region and Massachusetts: 1990-2005
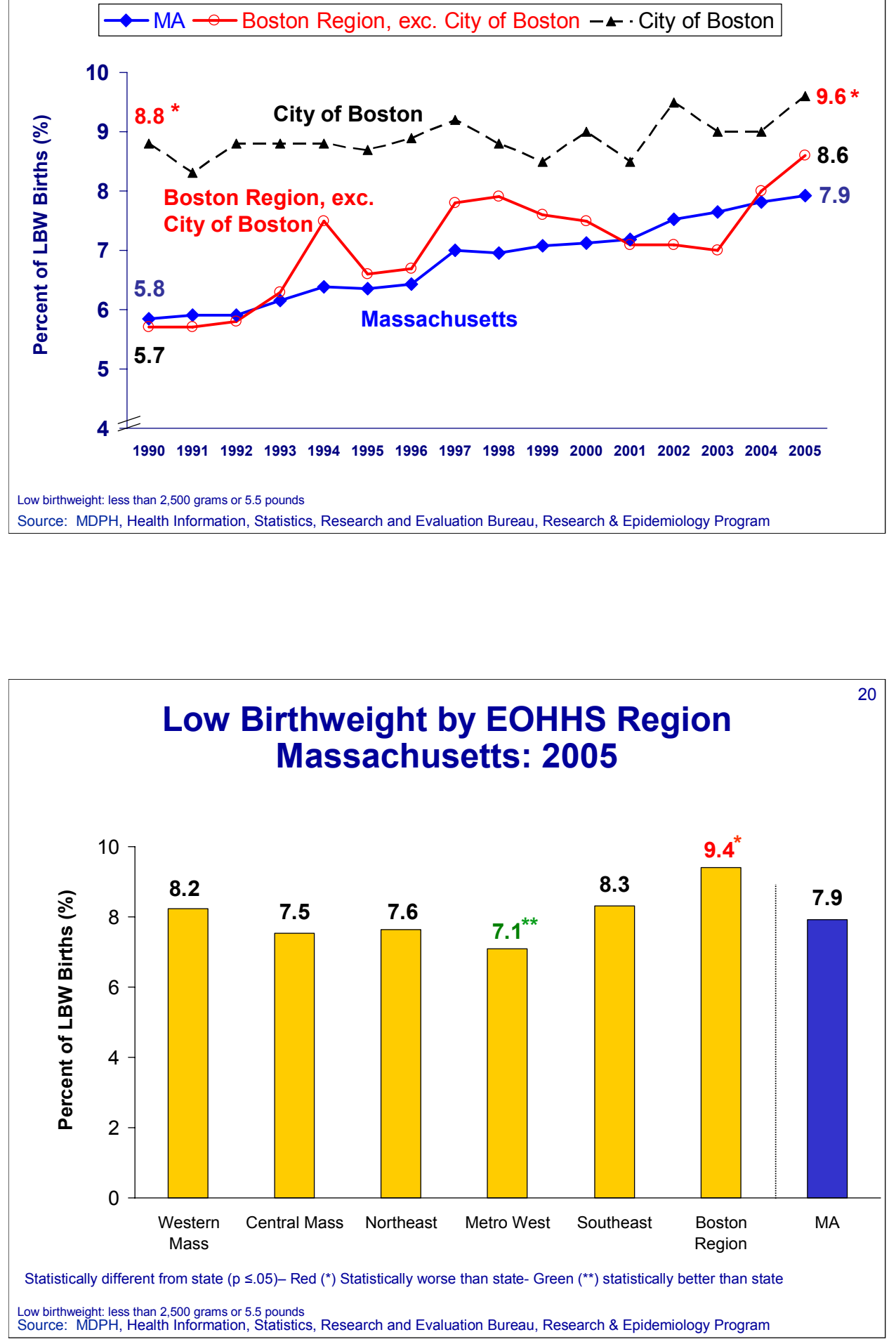


\section{Low Birthweight by Cities in Boston Region and Massachusetts: 2005}

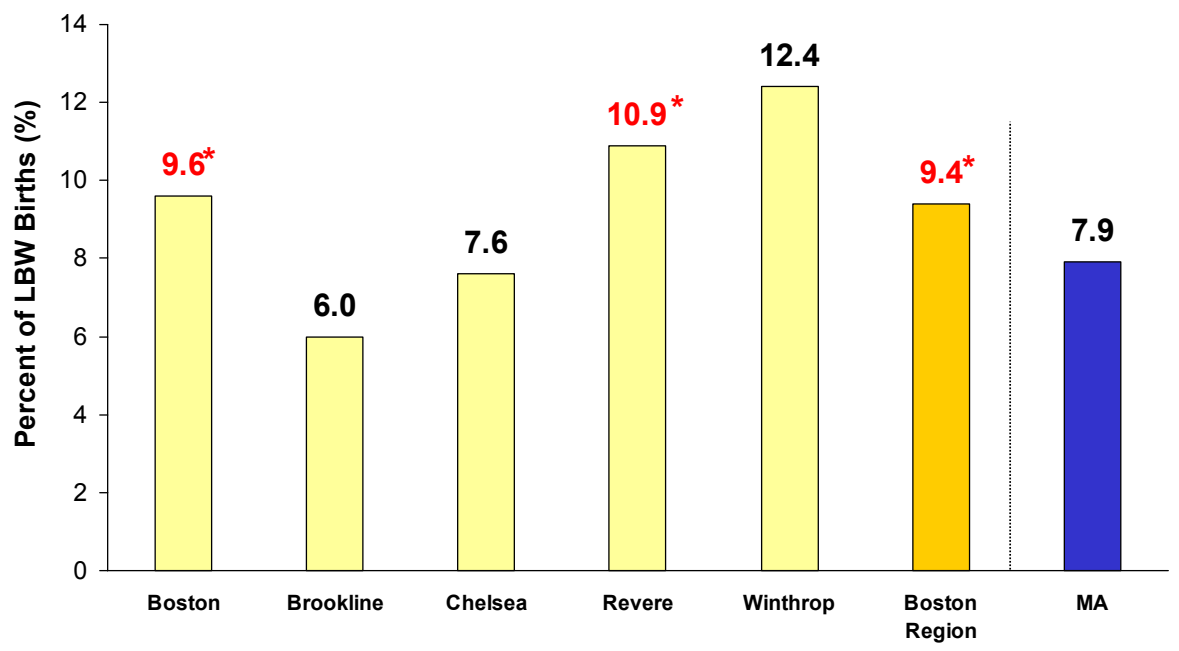

Statistically different from state $(p \leq .05)-$ Red $\left(^{*}\right)$ Statistically worse than state- Green $\left(^{* \star}\right)$ statistically better than state

\section{Breastfeeding by EOHHS Region Massachusetts: 2005}

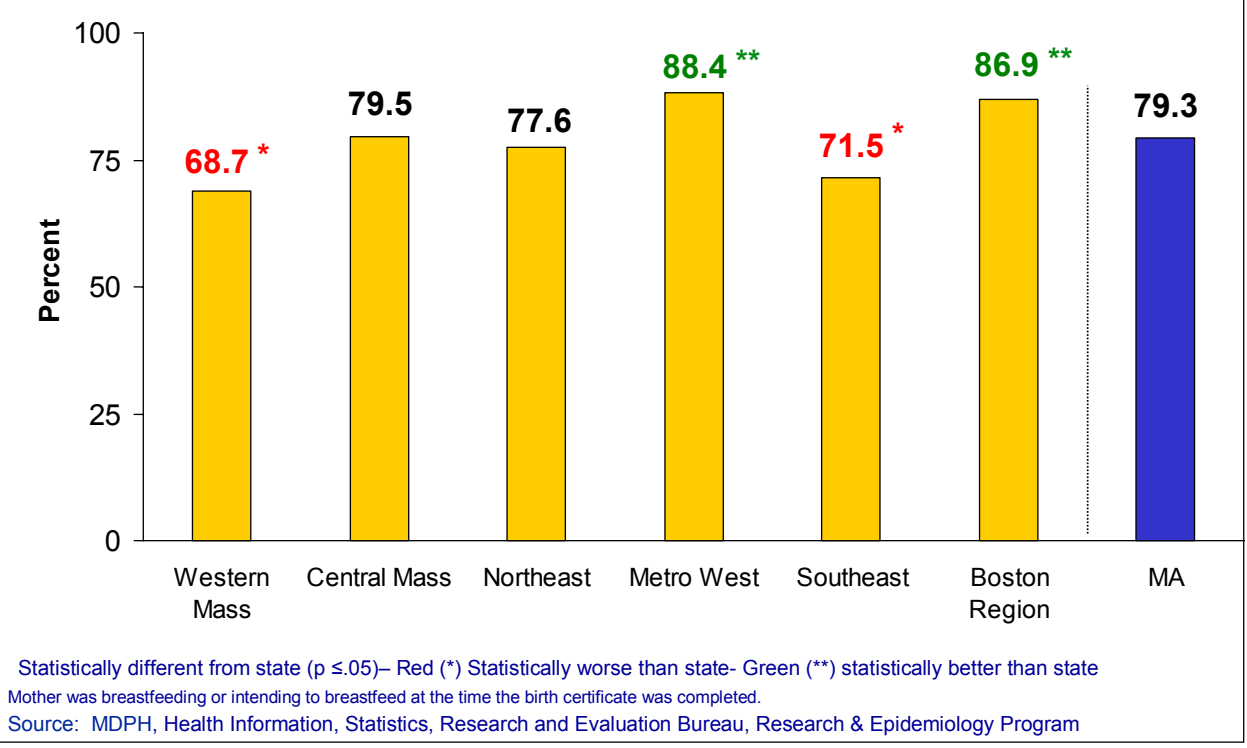


Teen Birth Rate

Boston Region and Massachusetts: 1990-2005

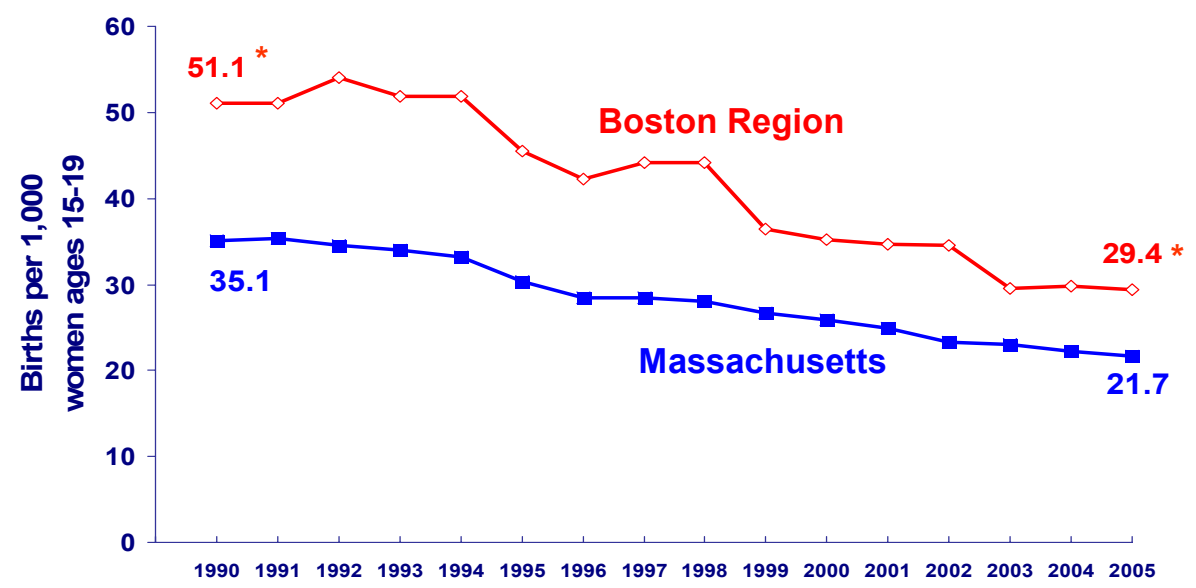

Statistically different from state $(\mathrm{p} \leq .05)-\operatorname{Red}\left({ }^{*}\right)$ Statistically higher than state- Green $\left(^{\star *}\right)$ statistically lower than state Teen birth rates are number of births to women ages 15-19 per 1000 females ages 15-19.

Source: MDPH, Health Information, Statistics, Research and Evaluation Bureau, Research \& Epidemiology Program

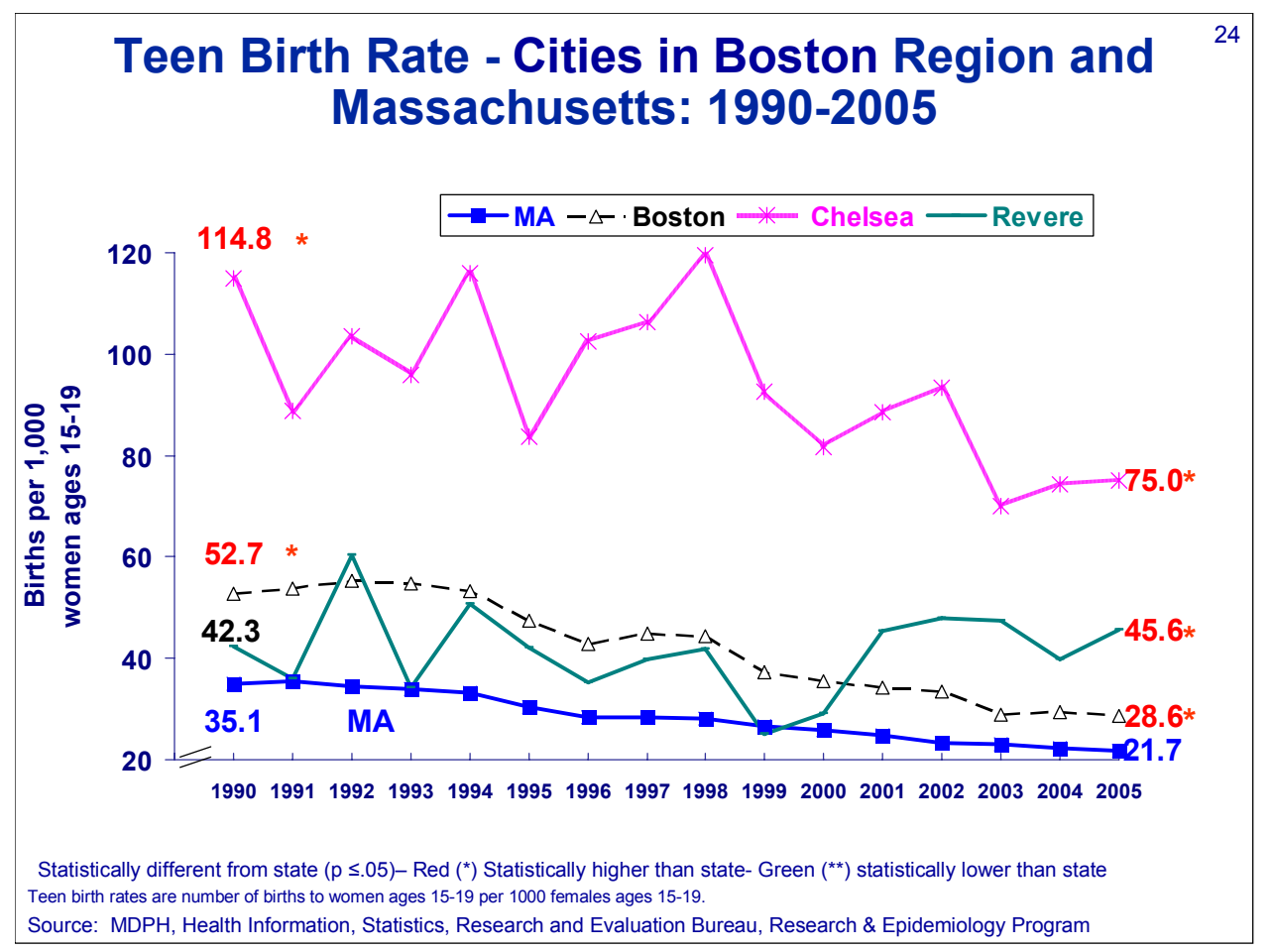


Teen Birth Rates by

Cities in Boston Region \& Massachusetts: 2003-2005

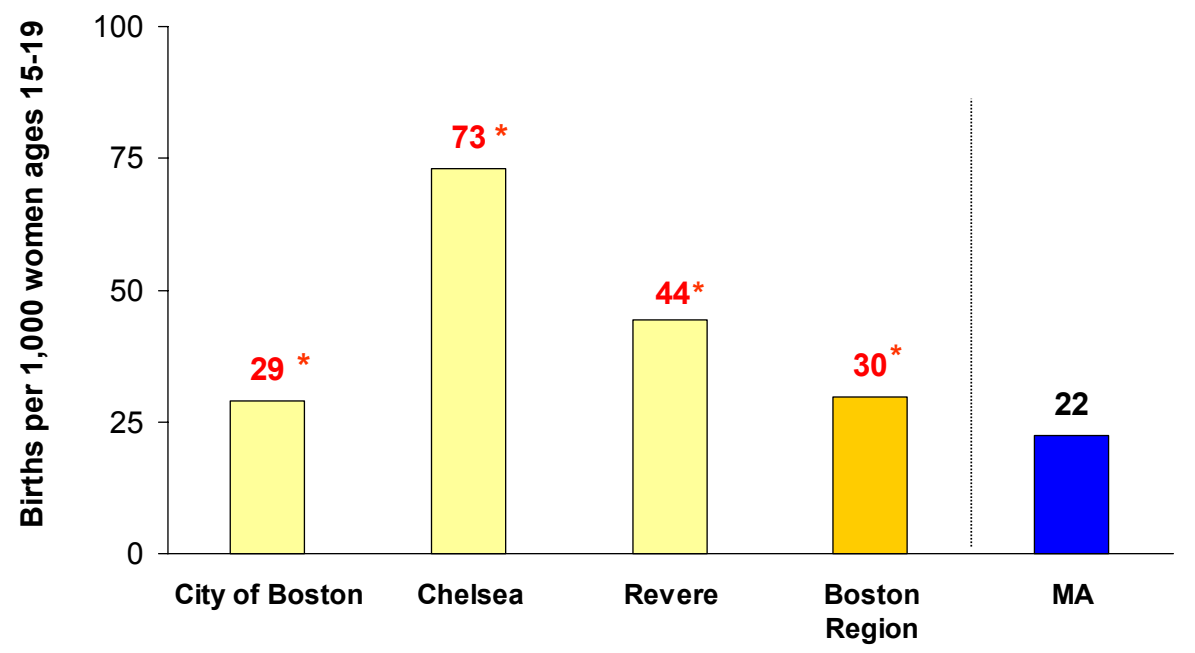

Statistically different from state $(p \leq .05)-$ Red $(*)$ Statistically worse than state- Green $\left(^{* *}\right)$ statistically better than state Teen birth rates are number of births to women ages $15-19$ per 1000 females ages $15-19$

Source: MDPH, Health Information, Statistics, Research and Evaluation Bureau, Research \& Epidemiology Program

\section{Teen Birth Rates by EOHHS Regions Massachusetts: 2005}

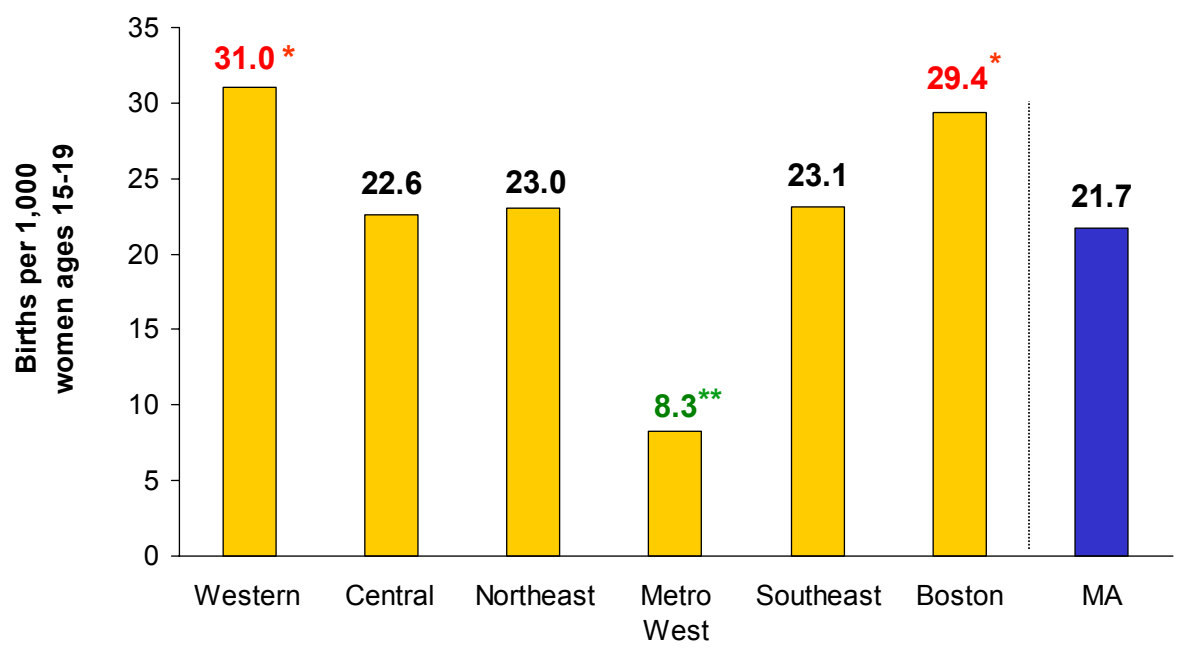

Statistically different from state $(p \leq .05)$ - Red $\left({ }^{*}\right)$ Statistically worse than state- Green $\left(^{* *}\right)$ statistically better than state Teen birth rates are number of births to women ages 15-19 per 1000 females ages 15-19.

Source: MDPH, Health Information, Statistics, Research and Evaluation Bureau, Research \& Epidemiology Program 


\section{Teen Birth Rates by EOHHS Region Massachusetts: 2005}

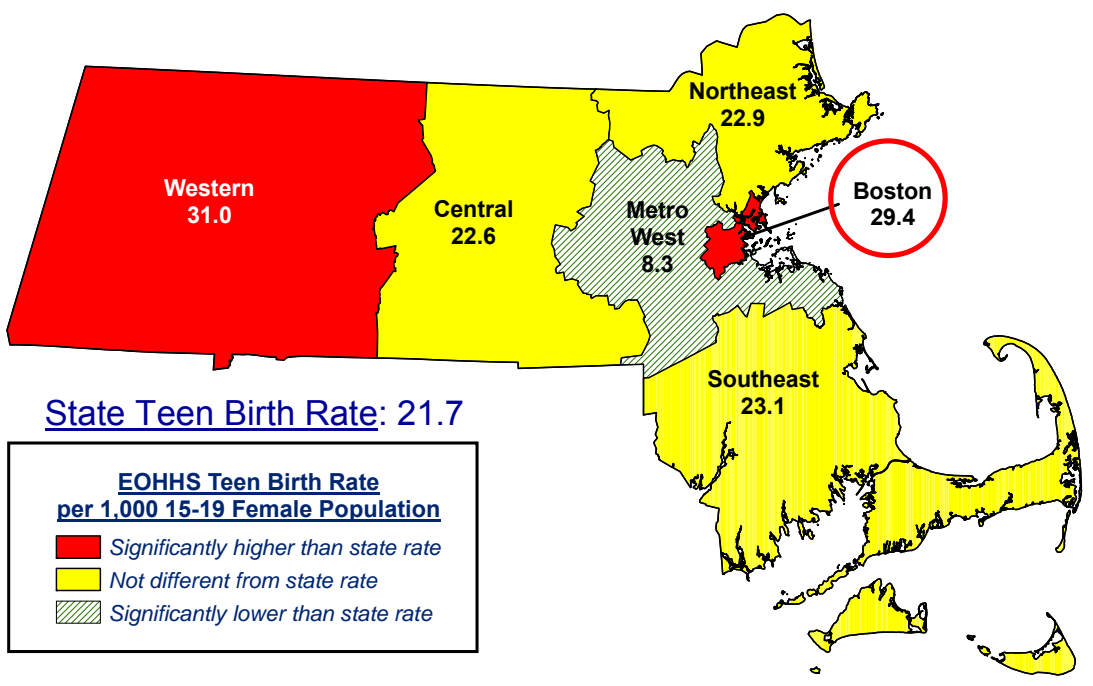

Teen birth rates are number of births to women ages 15-19 per 1000 females ages 15-19.

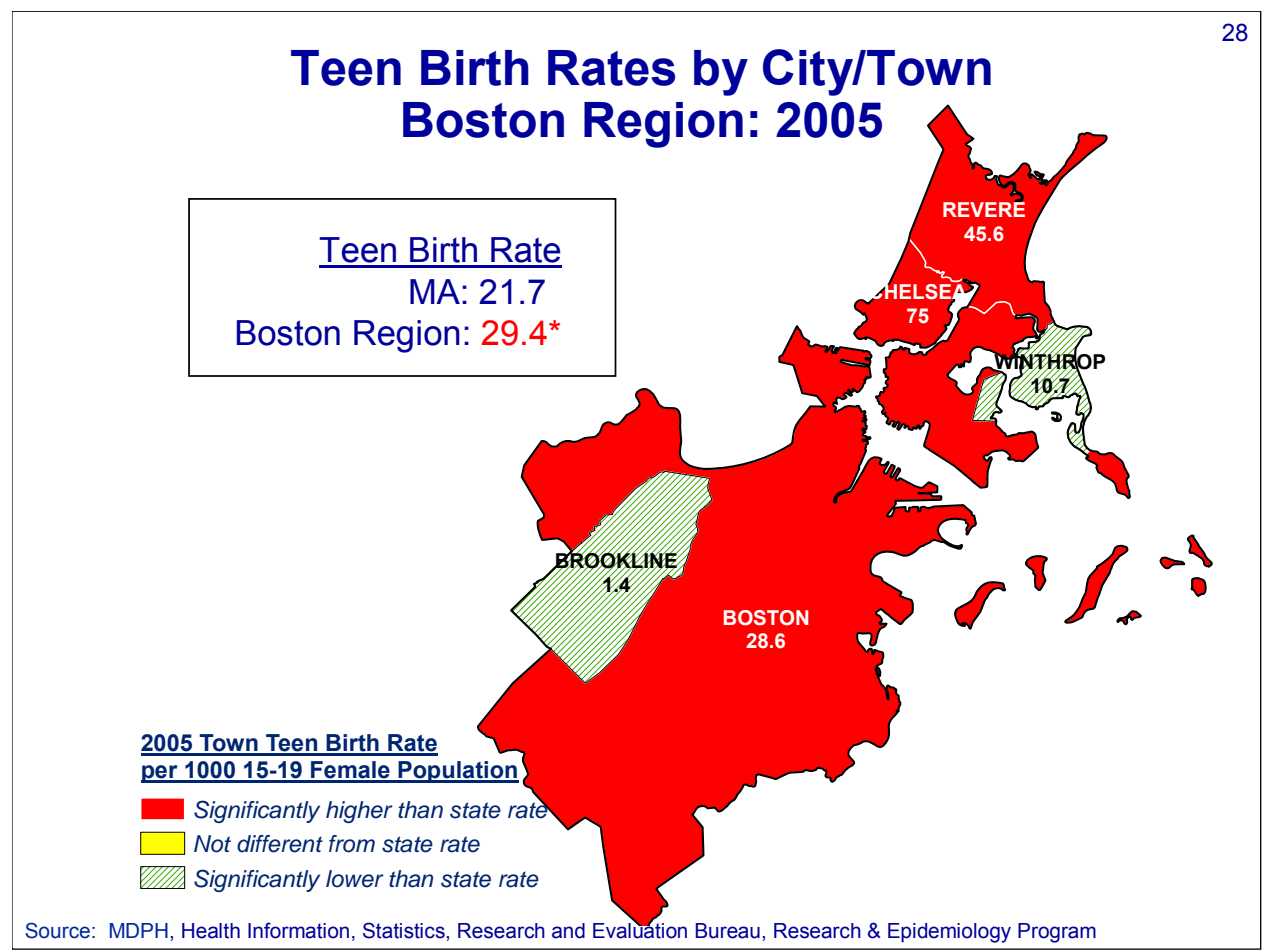




\section{Teen Birth Rates by Race and Ethnicity Boston Region and Massachusetts: 2005}

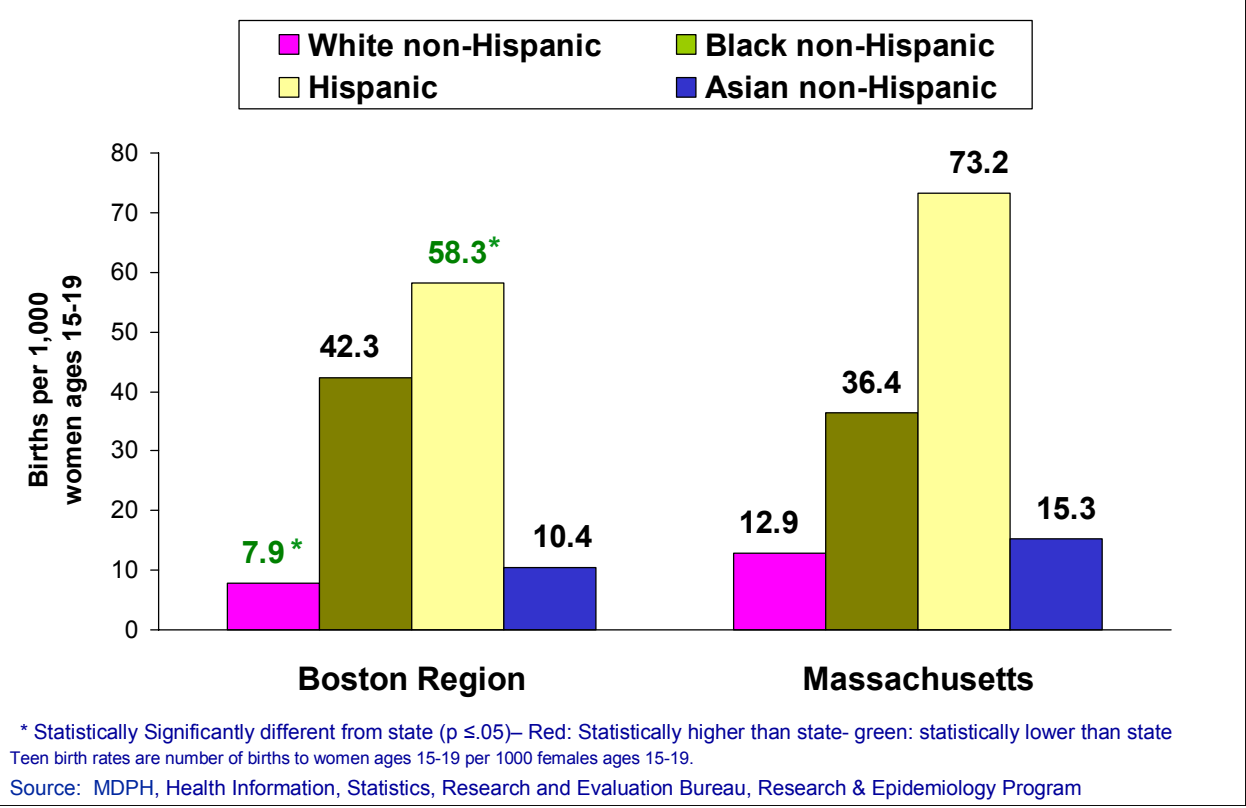

\section{Teen Birth Rate by Race and Ethnicity Cities in Boston Region \& Massachusetts: 2003-2005}

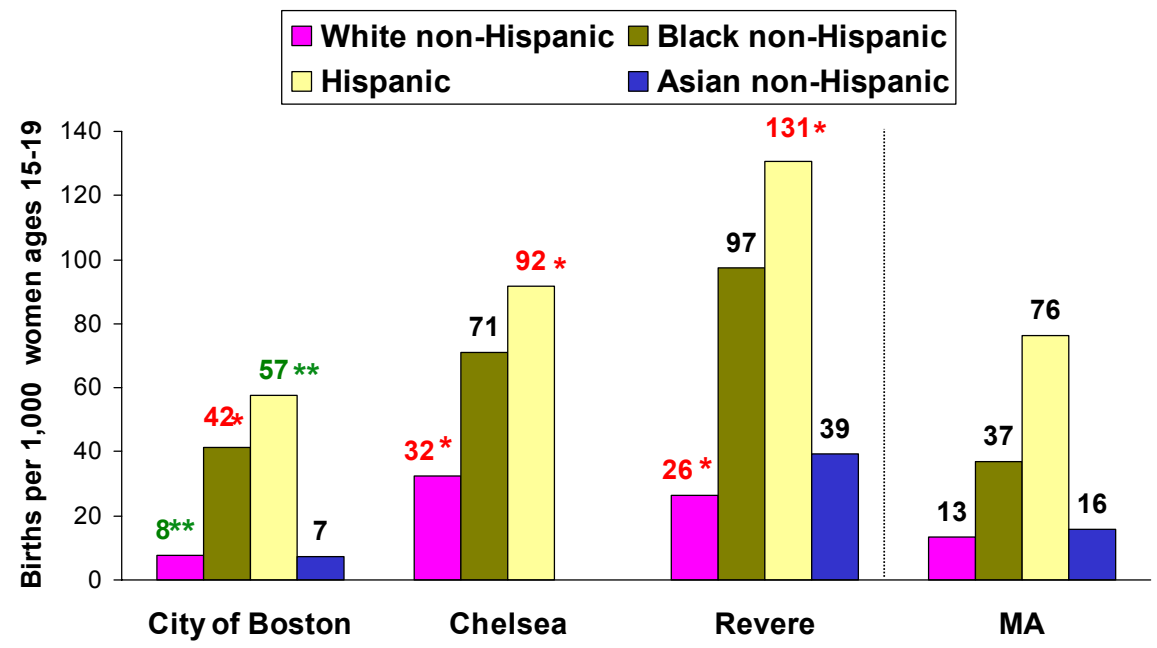

Statistically different from state $(p \leq .05)-$ Red $\left(^{*}\right)$ Statistically higher than state- Green $\left(^{* *}\right)$ statistically lower than state Teen birth rates are number of births to women ages 15-19 per 1000 females ages 15-19.

Source: MDPH, Health Information, Statistics, Research and Evaluation Bureau, Research \& Epidemiology Program 
Boston Region and Massachusetts: 1990-2005
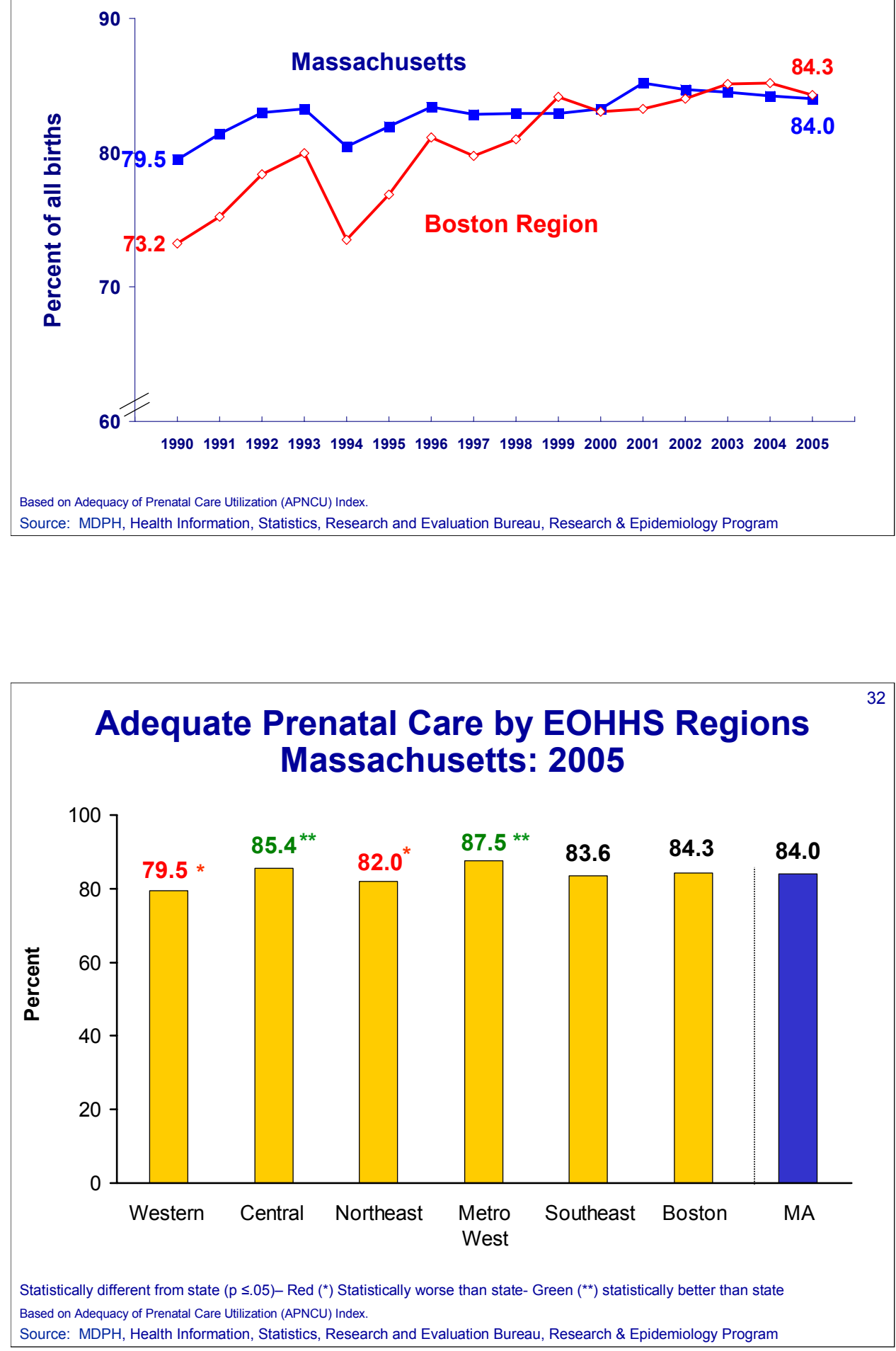


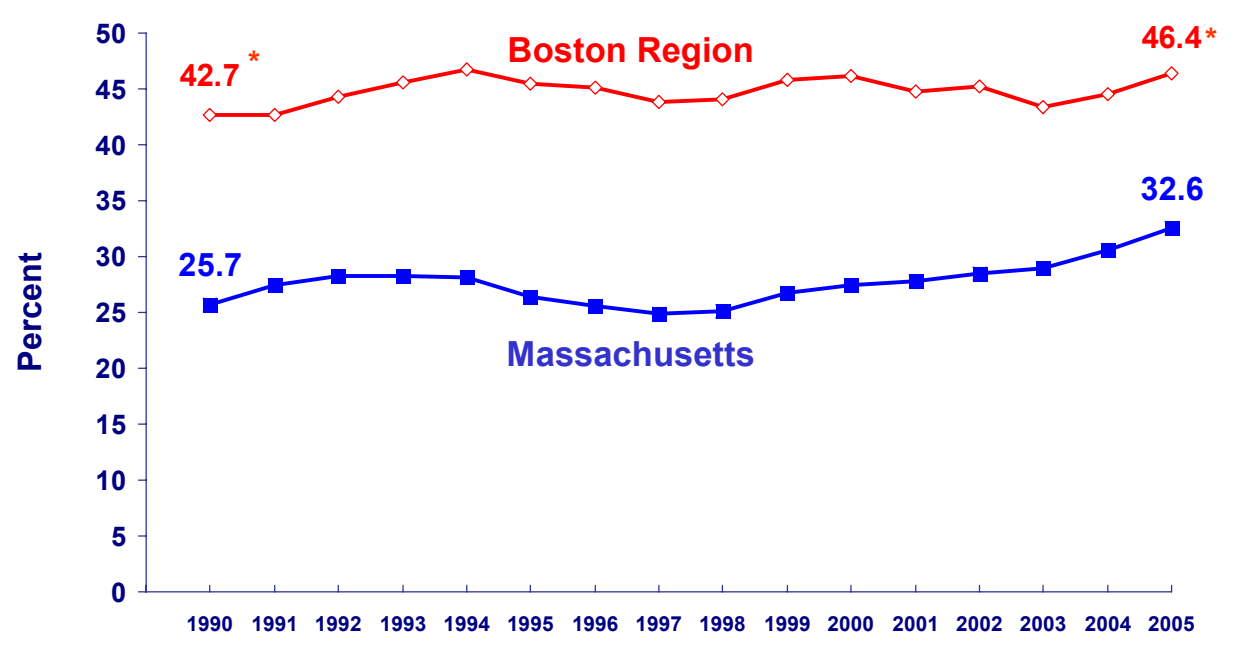

${ }^{1}$ Public: Government programs including Commonhealth, Healthy Start, Medicaid/MassHealth, or free care. Private: Commercial indemnity plan, commercial Managed care (HMO, PPO, IPP, IPA and other) or other private insurance.

Source: MDPH, Health Information, Statistics, Research and Evaluation Bureau, Research \& Epidemiology Program

\section{Public Source of Payment for Prenatal Care ${ }^{1}$ Boston Region and Massachusetts: 2005}

\section{Boston Region}

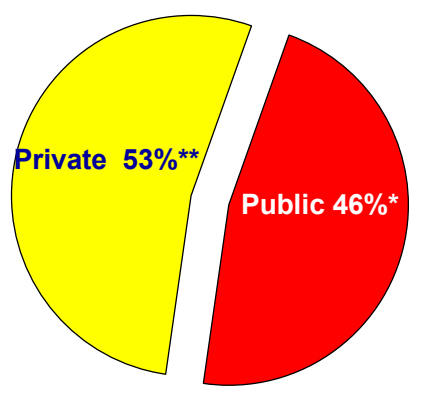

\section{Massachusetts}

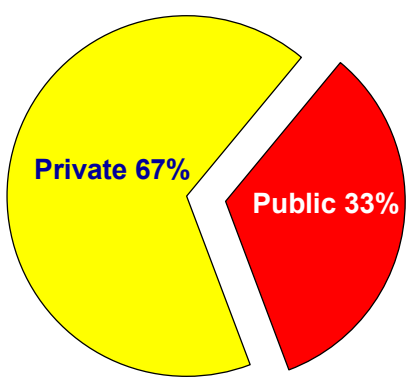

${ }^{1}$ Public: Government programs including Commonhealth, Healthy Start, Medicaid/MassHealth, or free care. Private: Commercial indemnity plan, commercial Managed care (HMO, PPO, IPP, IPA and other) or other private insurance.

Source: MDPH, Health Information, Statistics, Research and Evaluation Bureau, Research \& Epidemiology Program 

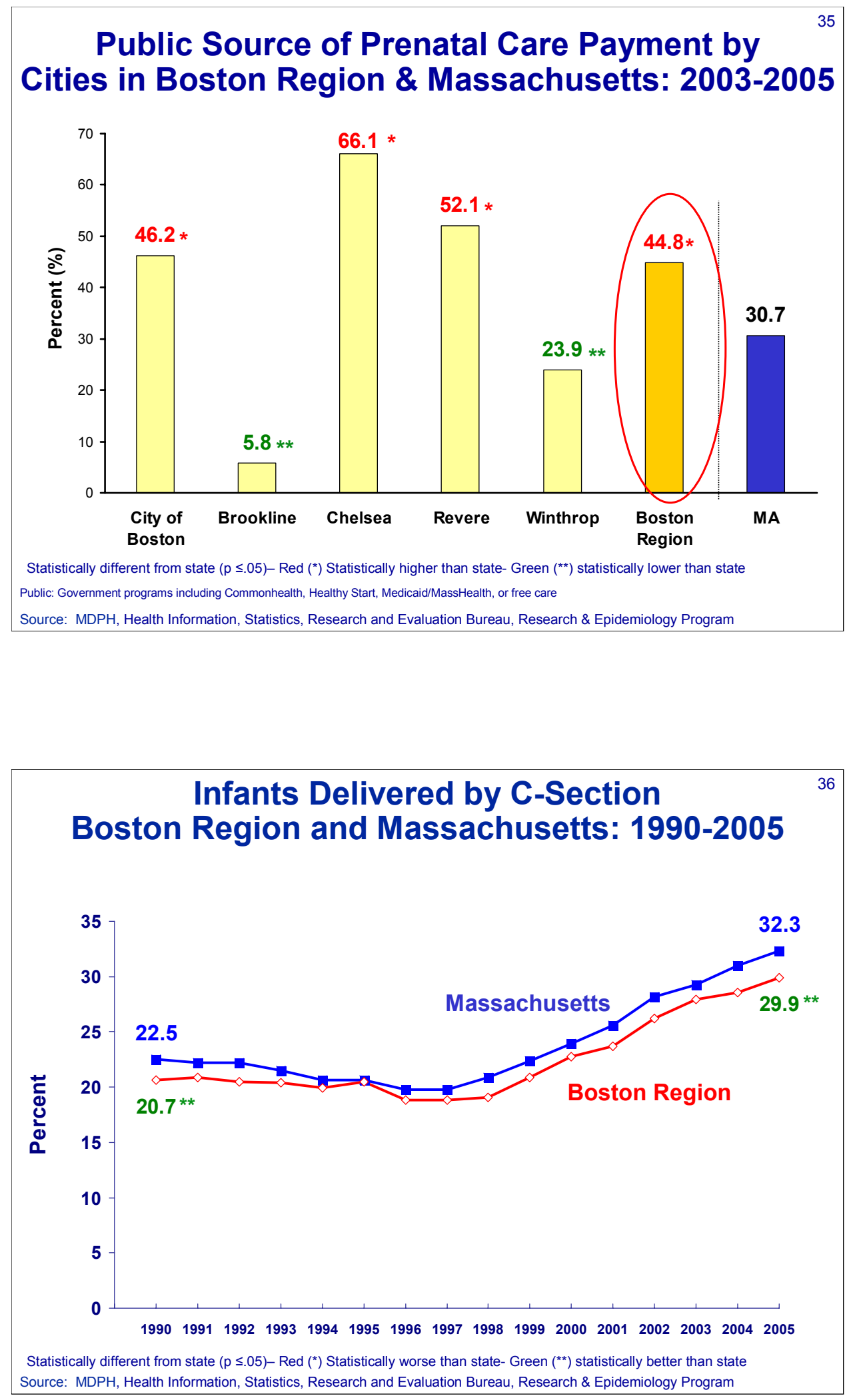


\section{Infants Delivered by C-Section by EOHHS Region, Massachusetts: 2005}

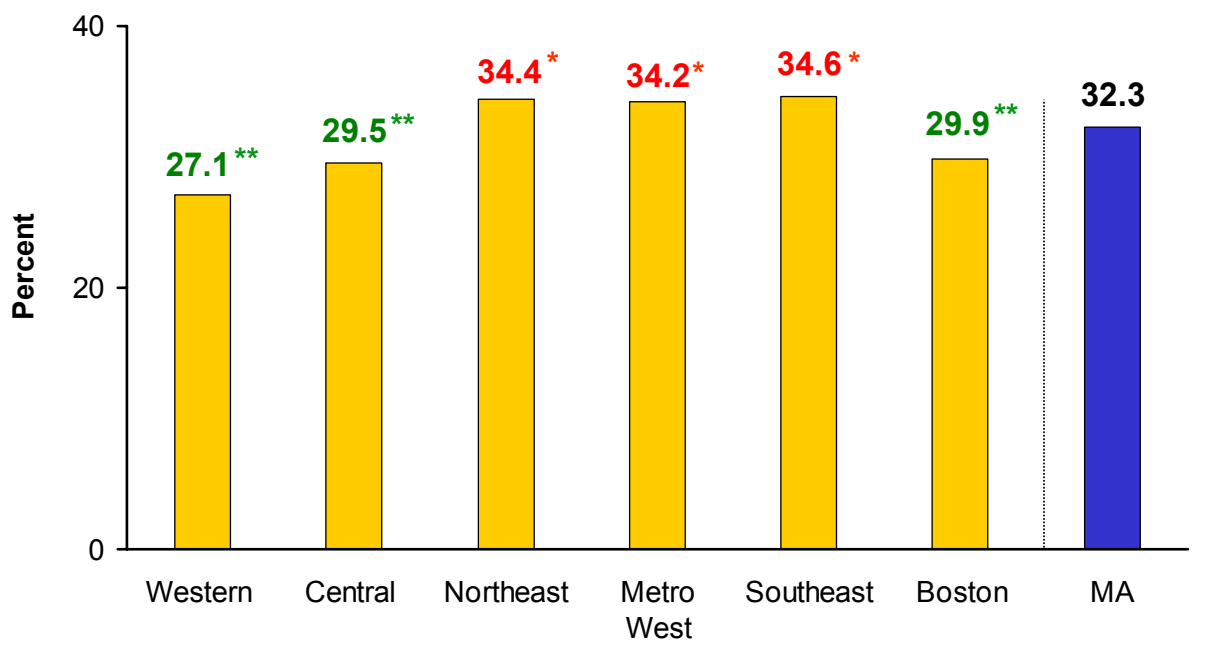

Statistically different from state $(p \leq .05)-\operatorname{Red}\left({ }^{*}\right)$ Statistically worse than state- Green $\left(^{* *}\right)$ statistically better than state

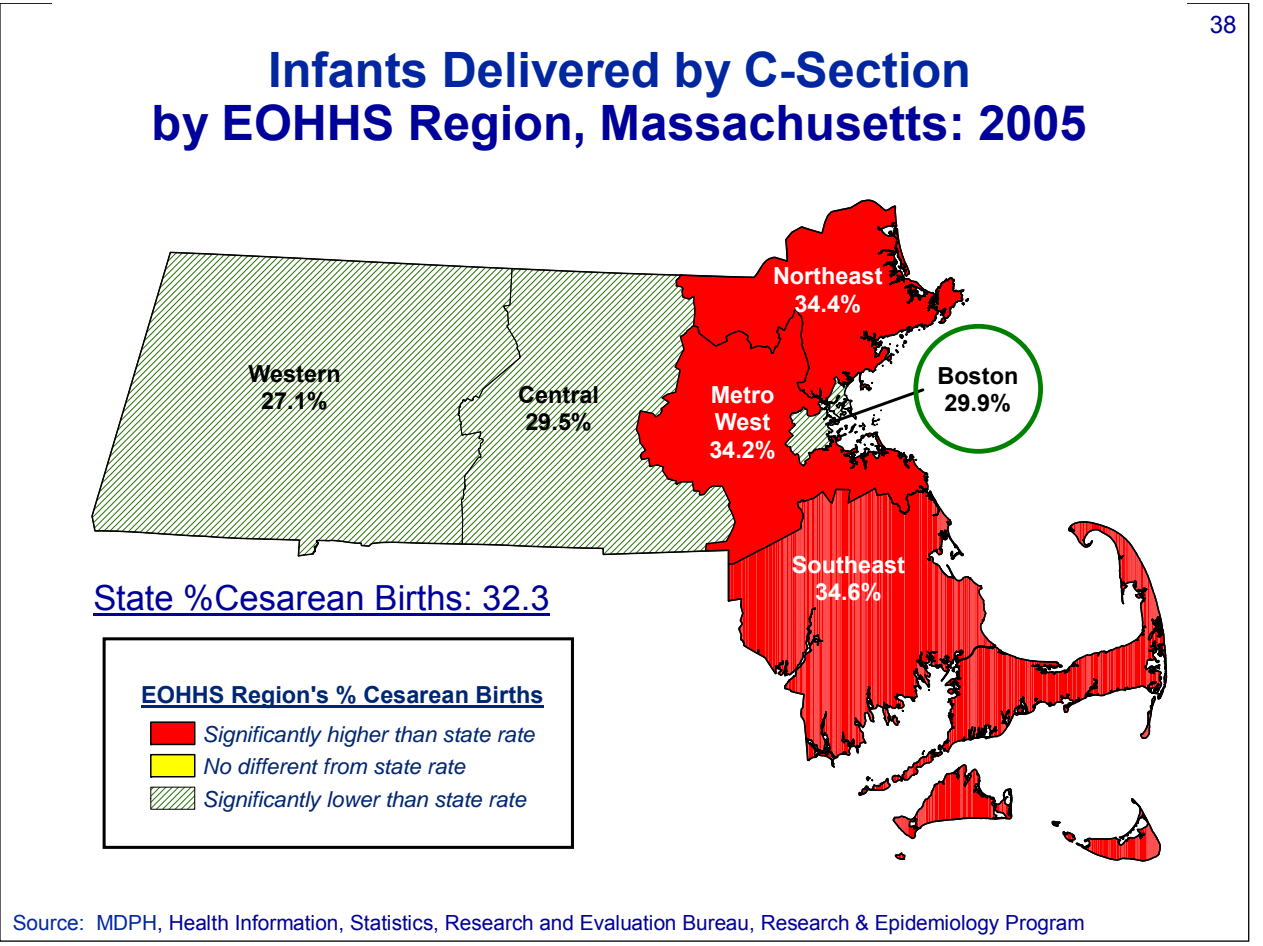




\section{Percent of Cesarean Deliveries by City/Town Boston Region: 2005}

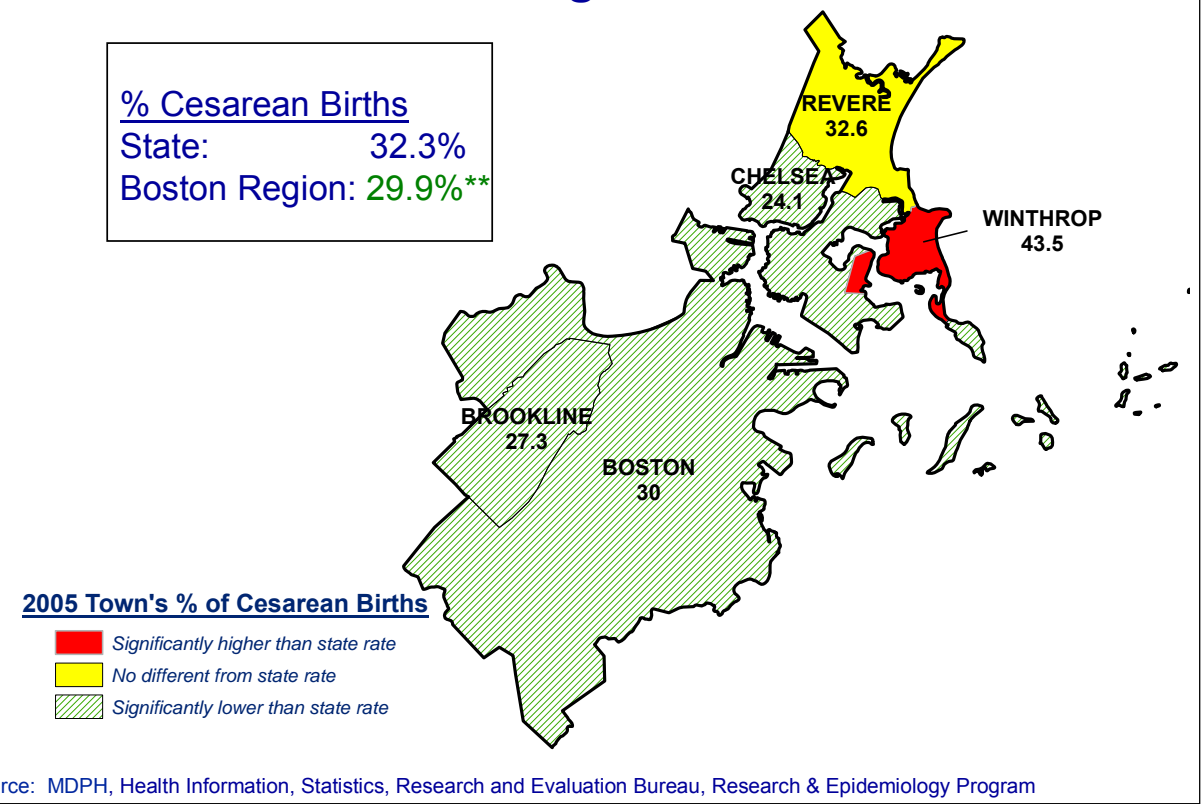

Source: MDPH, Health Information, Statistics, Research and Evaluation Bureau, Research \& Epidemiology Program

\section{Summary Birth Indicators by EOHHS Region, Massachusetts:2005}

\begin{tabular}{|c|c|c|c|c|c|c|c|}
\hline $\begin{array}{l}\text { Indicator } \\
\text { (as \% of race } \\
\text { group births) }\end{array}$ & $\begin{array}{c}\text { MA } \\
\text { Total } \\
(n=76,824)\end{array}$ & $\begin{array}{l}\text { Western } \\
(n=8,935)\end{array}$ & $\begin{array}{l}\text { Central } \\
(n=10,532)\end{array}$ & $\begin{array}{c}\text { Northeast } \\
(n=15,939)\end{array}$ & $\begin{array}{c}\text { Metro } \\
\text { West } \\
(n=17,093)\end{array}$ & $\begin{array}{l}\text { South } \\
\text { East } \\
(n=14,681)\end{array}$ & $\begin{array}{l}\text { Boston } \\
(n=9,644)\end{array}$ \\
\hline $\begin{array}{c}\text { Adequate } \\
\text { Prenatal Care } \\
\text { (Kotelchuck index) }\end{array}$ & 84.0 & 79.5 & 85.4 & 82.0 & 87.5 & 83.6 & 84.3 \\
\hline $\begin{array}{l}\text { C-section } \\
\text { deliveries }\end{array}$ & 32.3 & 27.1 & 29.5 & 34.4 & 34.2 & 34.6 & 29.9 \\
\hline $\begin{array}{l}\text { Low Birth } \\
\text { weight }(<5.5 \mathrm{lb})\end{array}$ & 7.9 & 8.2 & 7.5 & 7.6 & 7.1 & 8.3 & 9.4 \\
\hline Breastfeeding & 79.3 & 68.7 & 79.5 & 77.6 & 88.4 & 71.5 & 86.9 \\
\hline $\begin{array}{l}\text { Public Pay for } \\
\text { Prenatal Care }\end{array}$ & 32.6 & 47.4 & 27.9 & 33.7 & 17.2 & 34.7 & 46.4 \\
\hline $\begin{array}{l}\text { Smoking } \\
\text { during } \\
\text { pregnancy }\end{array}$ & 7.1 & 13.5 & 7.6 & 7.2 & 3.2 & 10.2 & 3.9 \\
\hline \multicolumn{4}{|c|}{ Better Outcome (significantly different from state) } & \multicolumn{4}{|c|}{ Worse Outcome (significantly different from state) } \\
\hline
\end{tabular}




\section{Summary Birth Indicators by EOHHS Region}

\begin{tabular}{||c|c|c|c|c|c|c|c|}
\hline Indicator & $\begin{array}{c}\text { MA } \\
\text { Total }\end{array}$ & Western & Central & Northeast & $\begin{array}{c}\text { Metro } \\
\text { West }\end{array}$ & $\begin{array}{c}\text { South } \\
\text { East }\end{array}$ & Boston \\
\hline $\begin{array}{c}\text { Infant } \\
\text { Mortality } \\
\text { Rate } \\
\text { (2002-2005) } \\
\text { (infant deaths } \\
\text { per 1000 live } \\
\text { births) }\end{array}$ & 4.9 & 5.6 & 5.4 & 4.7 & 4.0 & 4.8 & 5.7 \\
\hline $\begin{array}{c}\text { Teen birth } \\
\text { rate } \\
\text { (2005) } \\
\text { (birth to women } \\
\text { ages 15-19 per } \\
\text { 1000 women } \\
\text { ages 15-19) }\end{array}$ & $\mathbf{2 1 . 7}$ & 31.0 & 22.9 & 23.0 & 8.3 & 23.1 & 29.4 \\
\hline \\
$\square$ Better Outcome (significantly different from state) \\
$\square$ Worse Outcome (significantly different from state) \\
Source: MDPH, Health Information, Statistics, Research and Evaluation Bureau, Research \& Epidemiology Program \\
\hline
\end{tabular}




\section{LEADING CAUSES OF DEATH}




\begin{tabular}{|c|c|c|c|c|c|}
\hline \multicolumn{3}{|c|}{$\frac{\text { Boston Region }}{\mathrm{N}=5,394}$} & \multicolumn{3}{|c|}{$\frac{\text { Massachusetts }}{\mathrm{N}=53,776}$} \\
\hline Cancer & 1,293 & $24.0 \%$ & Heart Disease & 13,248 & $24.6 \%$ \\
\hline Heart Disease & 1,180 & $21.9 \%^{* *}$ & Cancer & 13,159 & $24.5 \%$ \\
\hline All Injuries & 315 & $5.8 \%^{*}$ & Stroke & 2,979 & $5.5 \%$ \\
\hline Stroke & 292 & $5.4 \%$ & All Injuries & 2,657 & $4.9 \%$ \\
\hline $\begin{array}{l}\text { Chronic Lower } \\
\text { Respiratory Disease }\end{array}$ & 230 & $4.3 \%$ & $\begin{array}{l}\text { Chronic Lower } \\
\text { Respiratory Disease }\end{array}$ & 2,643 & $4.9 \%$ \\
\hline Influenza and Pneumonia & 212 & $3.9 \%$ & Influenza and Pneumonia & 1,932 & $3.6 \%$ \\
\hline Nephritis & 165 & $3.1 \%$ & Alzheimer's & 1,635 & $3.0 \%$ \\
\hline Septicemia & 140 & $2.6 \%{ }^{*}$ & Nephritis & 1,403 & $2.6 \%$ \\
\hline Diabetes & 139 & $2.6 \%$ & Diabetes & 1,271 & $2.4 \%$ \\
\hline Alzheimer's & 107 & $2.0 \%$ & Septicemia & 982 & $1.8 \%$ \\
\hline
\end{tabular}




\section{CHRONIC DISEASE OUTCOMES AND OVERWEIGHT/OBESITY}




\section{Thresholds for Defining Overweight and Obesity}

\begin{tabular}{|c|c|c|}
\hline Height & $\frac{\text { Overweight }}{\text { BMl 25.0-29.9 }}$ & $\frac{\text { Obesity }}{\mathrm{BMI}>30}$ \\
\hline 5 & $128 \mathrm{lbs}$. & 153 lbs. \\
\hline $5 ' 2 "$ & 136 & 164 \\
\hline 5'4"' & 145 & 174 \\
\hline 5'6" & 155 & 186 \\
\hline 5'8" & 164 & 197 \\
\hline $6^{\prime}$ & 184 & 221 \\
\hline 6'2" & 195 & 234 \\
\hline
\end{tabular}

\section{by EOHHS Region, Massachusetts: 1995 \& 2005}

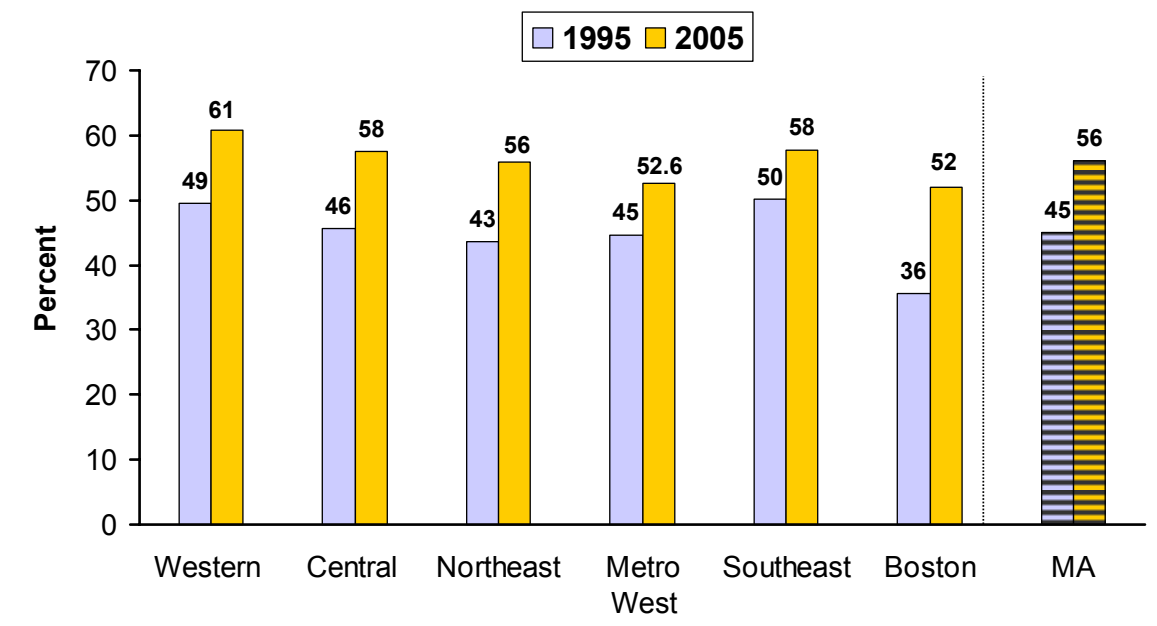

Statistically different from state $(p \leq .05)$ - Red $\left({ }^{*}\right)$ Statistically worse than state- Green $\left(^{* \star}\right)$ statistically better than state

1 Overweight: BMI greater than or equal to 25

Source: MDPH, Health Information, Statistics, Research and Evaluation Bureau, Massachusetts Behavioral Risk Factor Surveillance System (BRFSS), Health Survey Program 
Adults who are Overweight by Race and Ethnicity Boston Region: 1999-2005

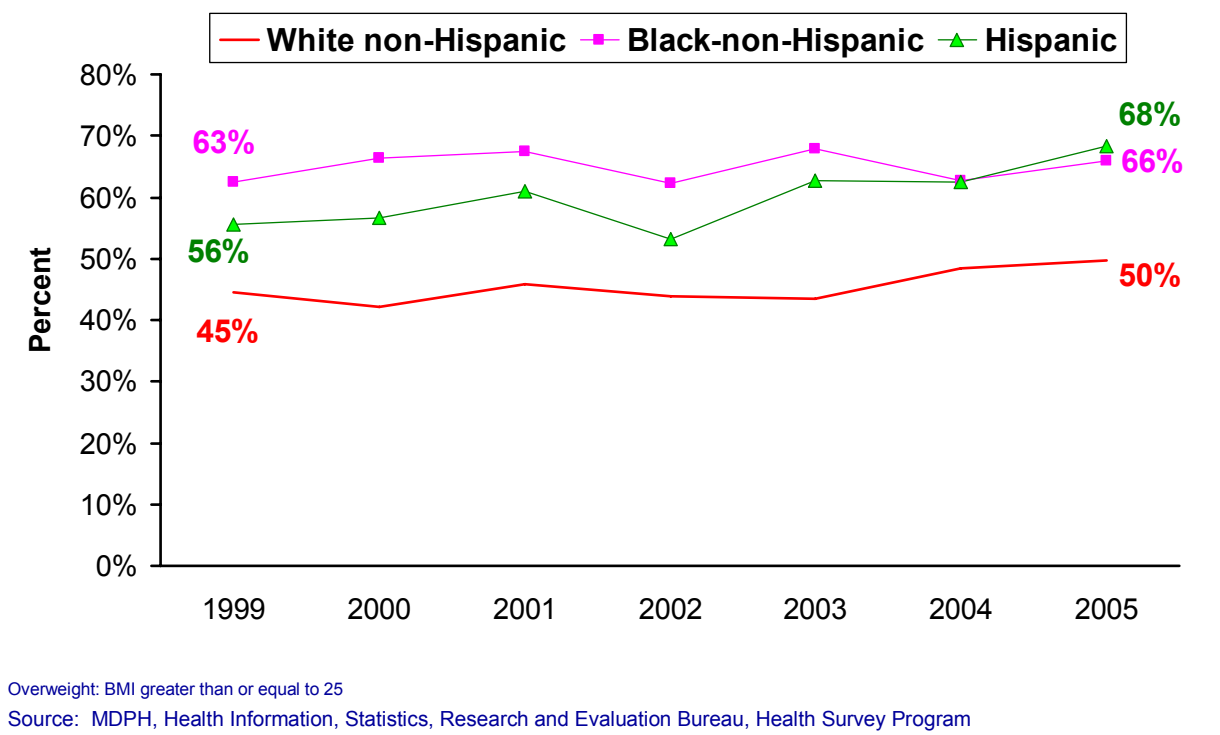

Source: MDPH, Health Information, Statistics, Research and Evaluation Bureau, Health Survey Program

\section{Adults who are Obese 1}

by EOHHS Region, Massachusetts: 1995 \& 2005

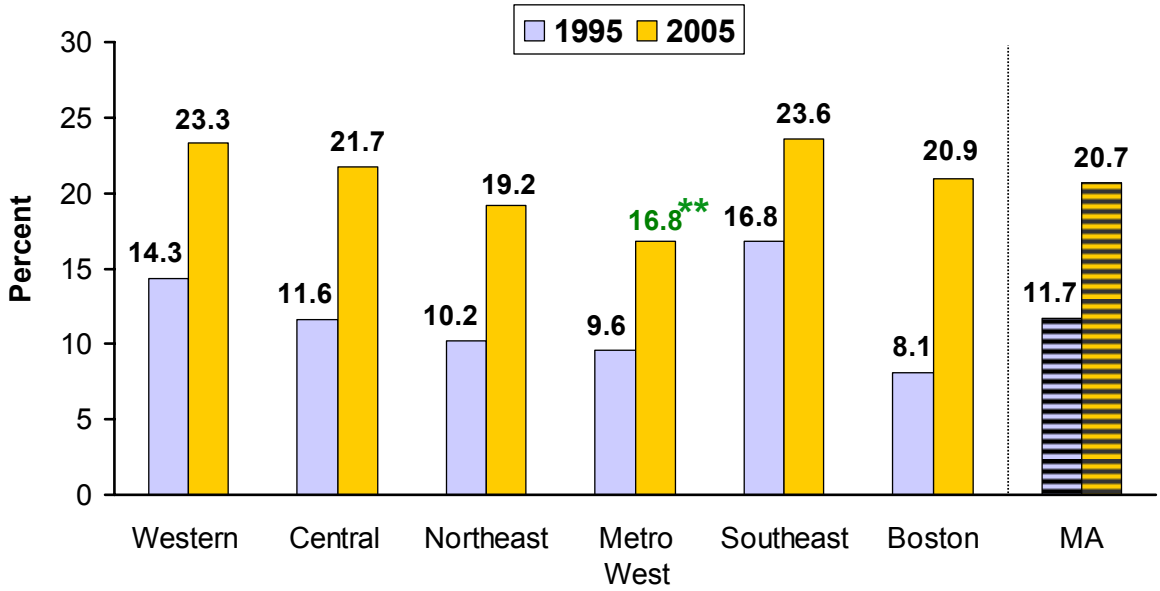

Statistically different from state $(p \leq .05)-$ Red $\left({ }^{*}\right)$ Statistically worse than state- Green $\left(^{* \star}\right)$ statistically better than state

1 Obesity: BMl greater than or equal to 30

Source: MDPH, Health Information, Statistics, Research and Evaluation Bureau, Massachusetts Behavioral Risk Factor Surveillance System (BRFSS), Health Survey Program 


\section{Adults who Participate in Regular Physical Activity by EOHHS Region, Massachusetts: 2005}

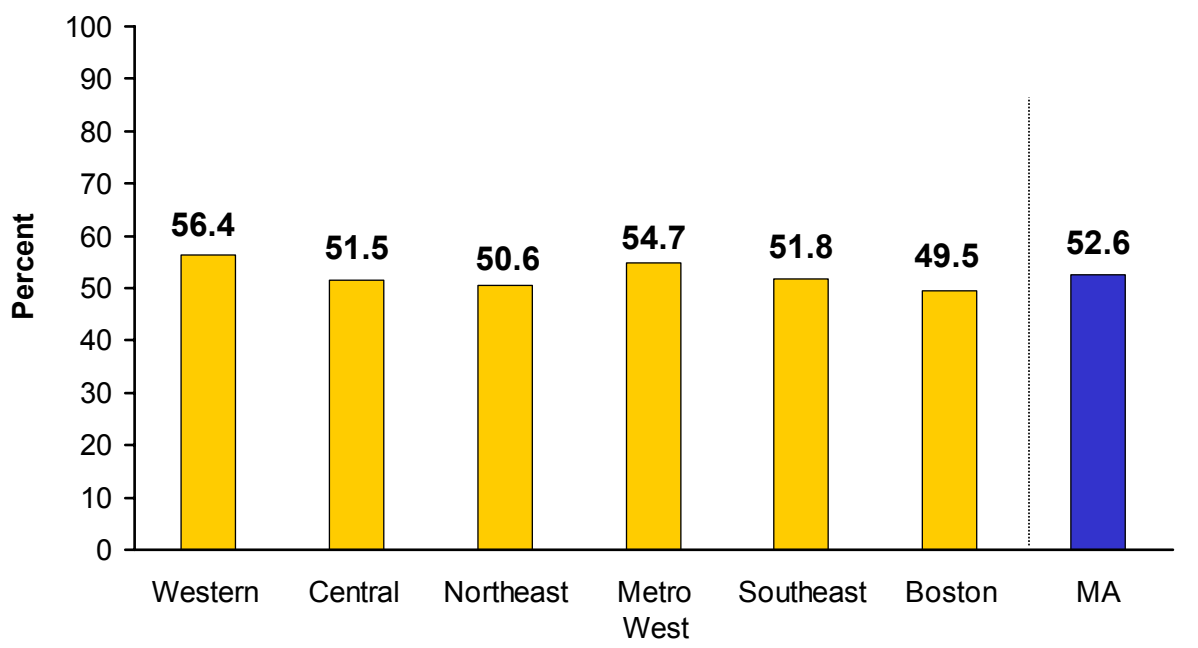

Statistically different from state $(p \leq .05)-\operatorname{Red}\left({ }^{*}\right)$ Statistically worse than state- Green $\left(^{* *}\right)$ statistically better than state Source: MDPH, Health Information, Statistics, Research and Evaluation Bureau, Health Survey Program

\section{Adults who have 5+ Servings of Fruit or Vegetables by EOHHS Region, Massachusetts: 2005}

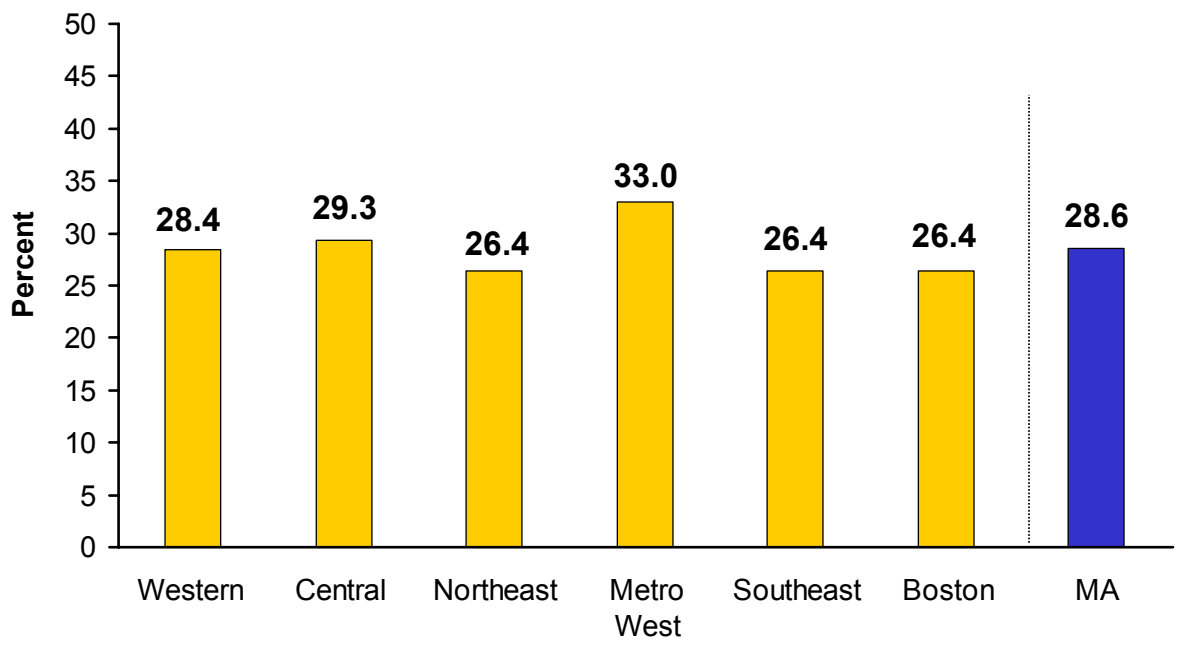

Statistically different from state $(p \leq .05)-\operatorname{Red}\left({ }^{*}\right)$ Statistically worse than state- Green $\left(^{* *}\right)$ statistically better than state

Source: MDPH, Health Information, Statistics, Research and Evaluation Bureau, Health Survey Program 
Adults who had their cholesterol checked in Past 5 Years by EOHHS Region Massachusetts: 2005

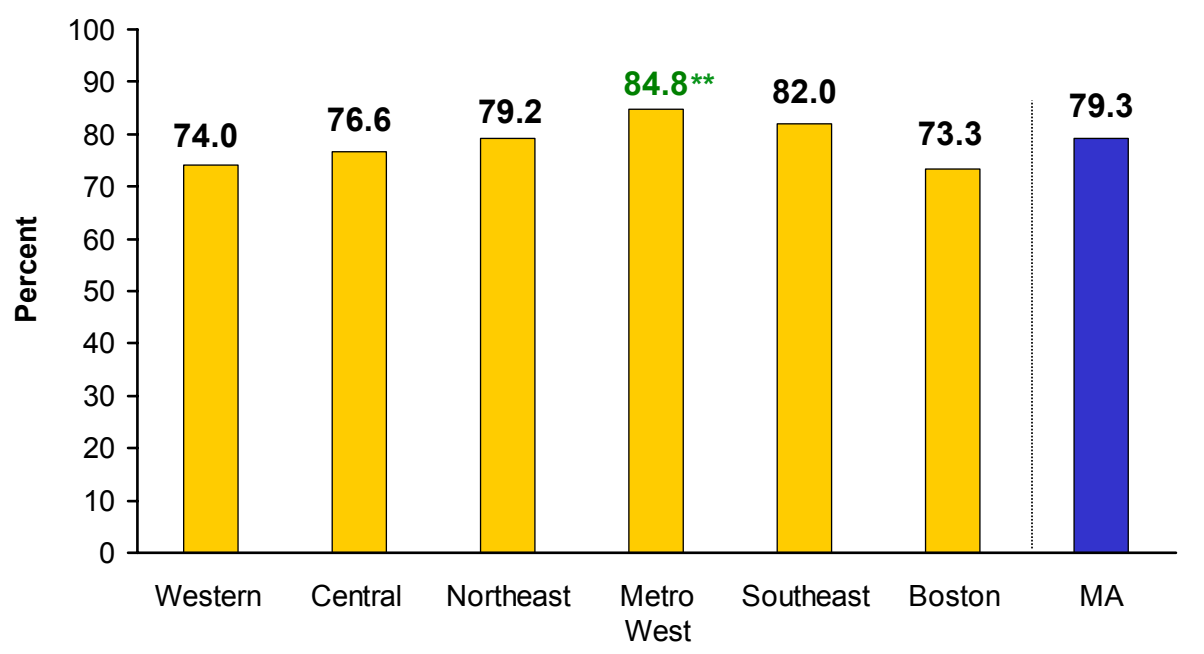

Statistically different from state $(p \leq .05)-\operatorname{Red}\left({ }^{*}\right)$ Statistically worse than state- Green $\left(^{* *}\right)$ statistically better than state Source: MDPH, Health Information, Statistics, Research and Evaluation Bureau, Health Survey Program

\section{Adults who were told by a health care provider that they have high cholesterol ${ }^{1}$ by EOHHS Region Massachusetts: 2005}

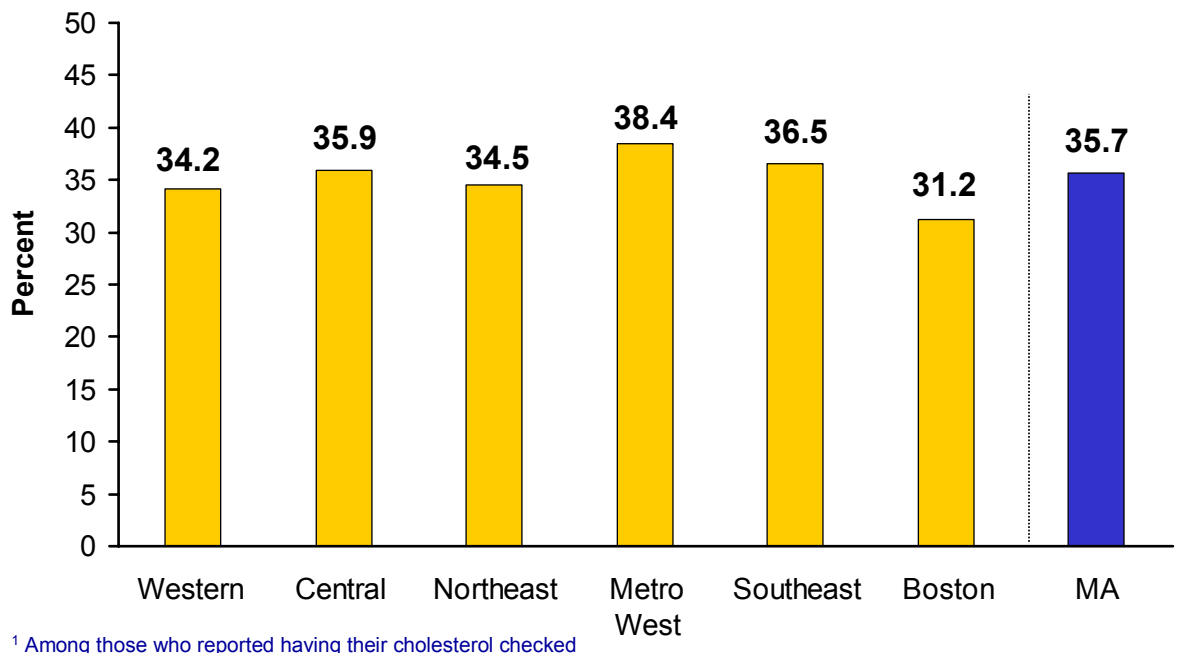

Statistically different from state ( $p \leq .05)-$ Red $\left(^{*}\right)$ Statistically worse than state- Green $\left(^{* *}\right)$ statistically better than state Source: MDPH, Health Information, Statistics, Research and Evaluation Bureau, Health Survey Program 


\section{Adults who were told by a health care provider to ${ }^{51}$ have high blood pressure by EOHHS Region Massachusetts: 2005}

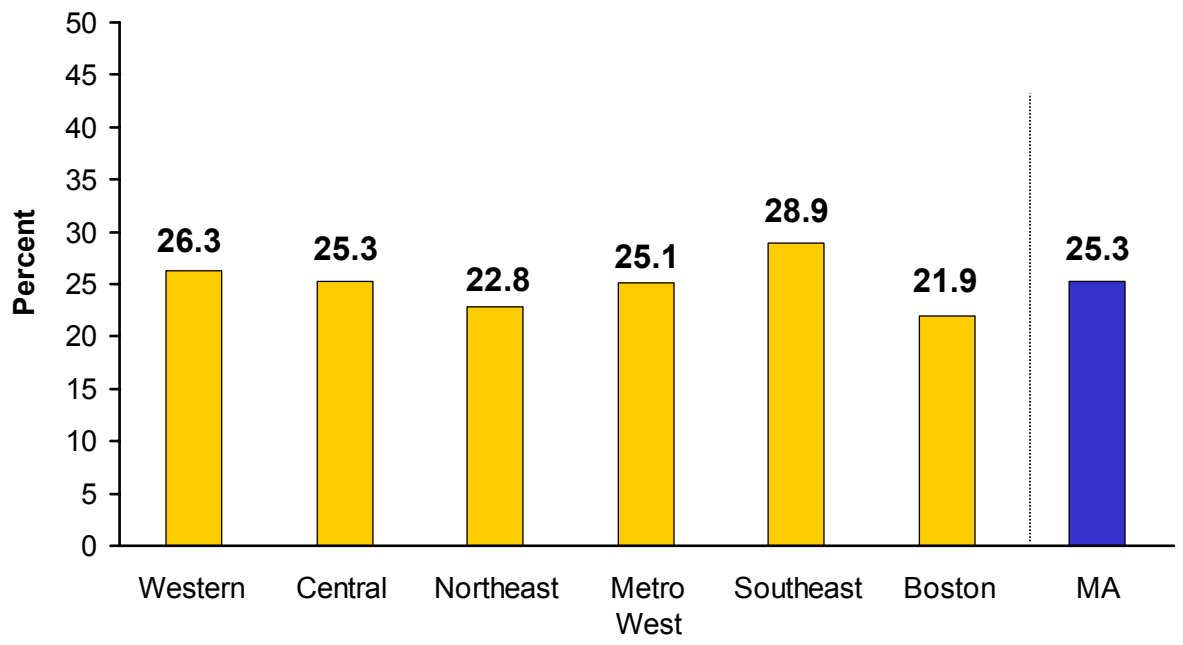

Statistically different from state $(p \leq .05)-\operatorname{Red}\left({ }^{*}\right)$ Statistically worse than state- Green $\left({ }^{* *}\right)$ statistically better than state Source: MDPH, Health Information, Statistics, Research and Evaluation Bureau, Health Survey Program

\section{Adults who take Medicine for High Blood Pressure ${ }^{1}$ by EOHHS Region Massachusetts: 2005}

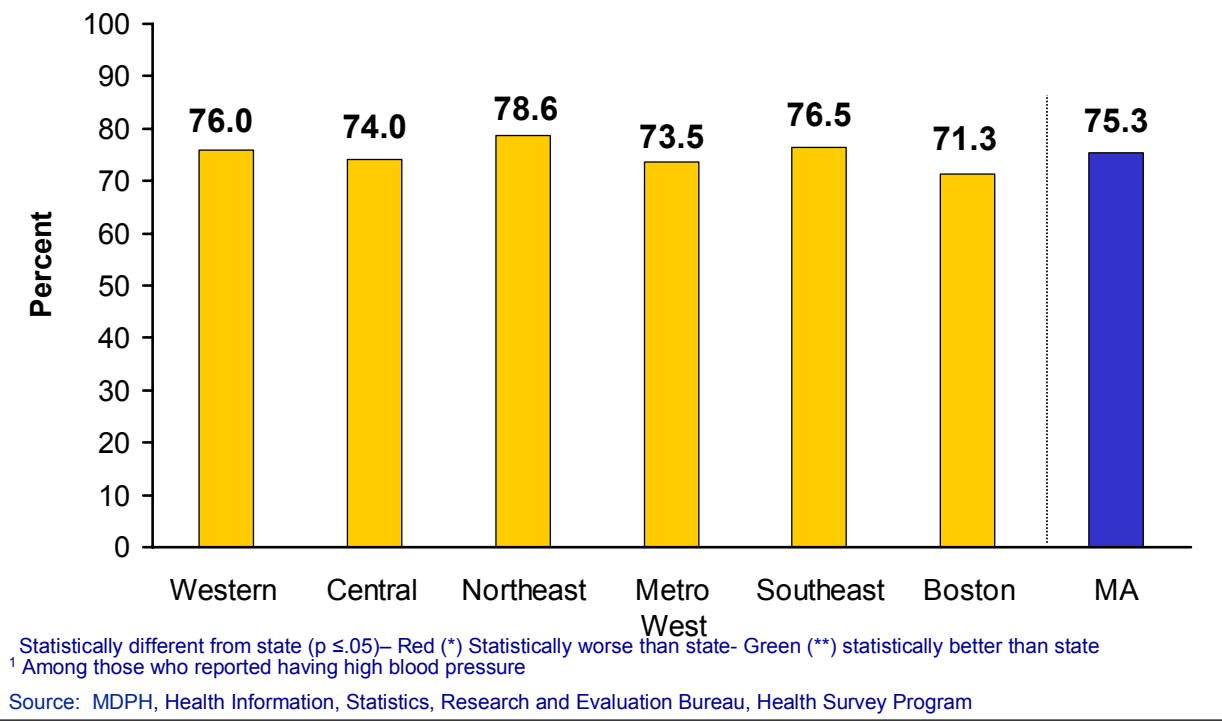


Hospital Discharge Rates for Hypertension
by EOHHS Regions and Massachusetts: 2003-2005

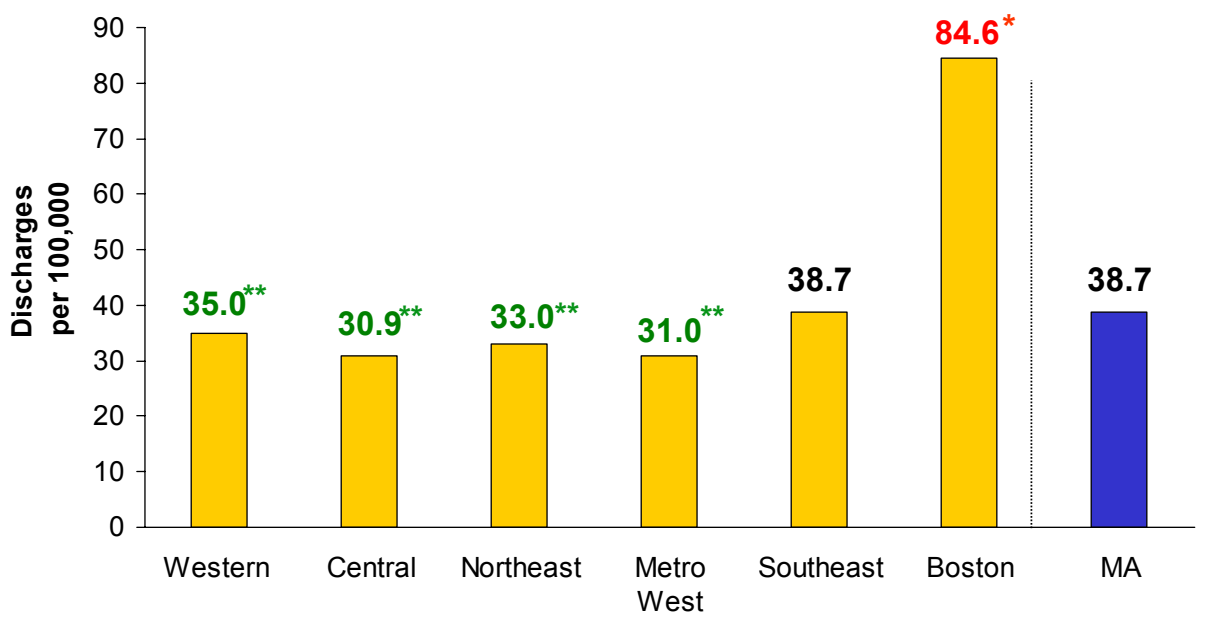

Statistically different from state $(p \leq .05)-\operatorname{Red}\left({ }^{*}\right)$ Statistically worse than state- Green $\left(^{* \star}\right)$ statistically better than state Age-adjusted to the 2000 US standard population.

Hospital Discharge Rates for Hypertension by Cities in Boston Region \& Massachusetts: 2003-2005

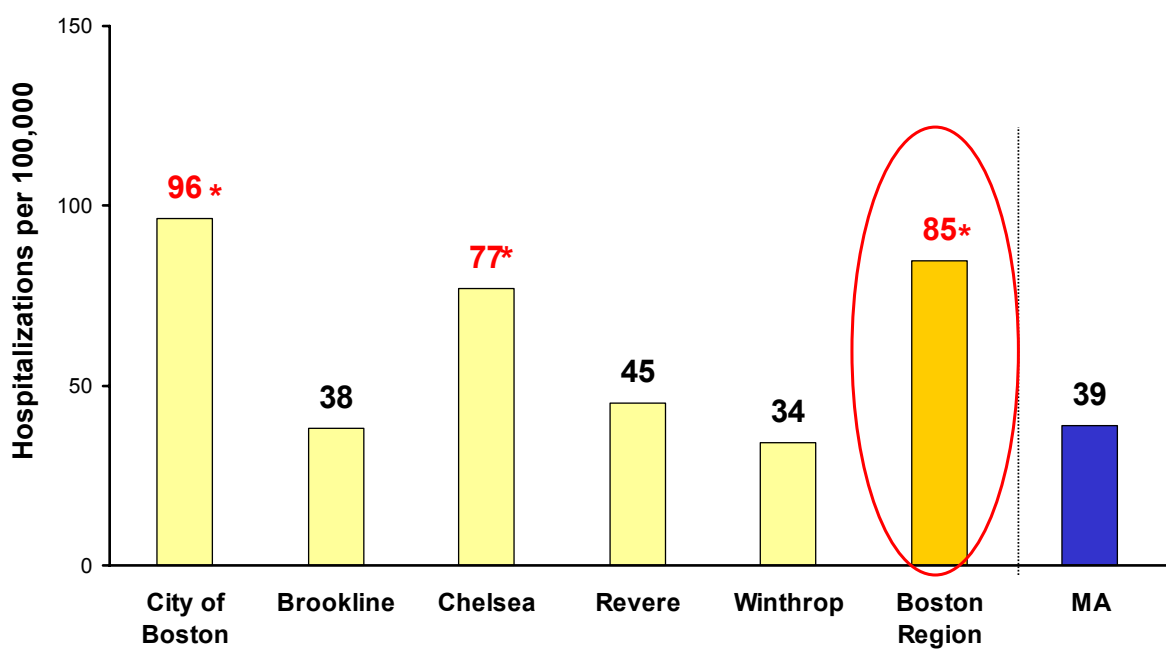

Statistically different from state $(p \leq .05)-\operatorname{Red}\left({ }^{*}\right)$ Statistically worse than state- Green $\left({ }^{* *}\right)$ statistically better than state Age-adjusted to the 2000 US standard population.

Source: Division of Health Care Finance and Policy. Calendar Year 2003-2005. Inpatient Hospital Discharge Database 

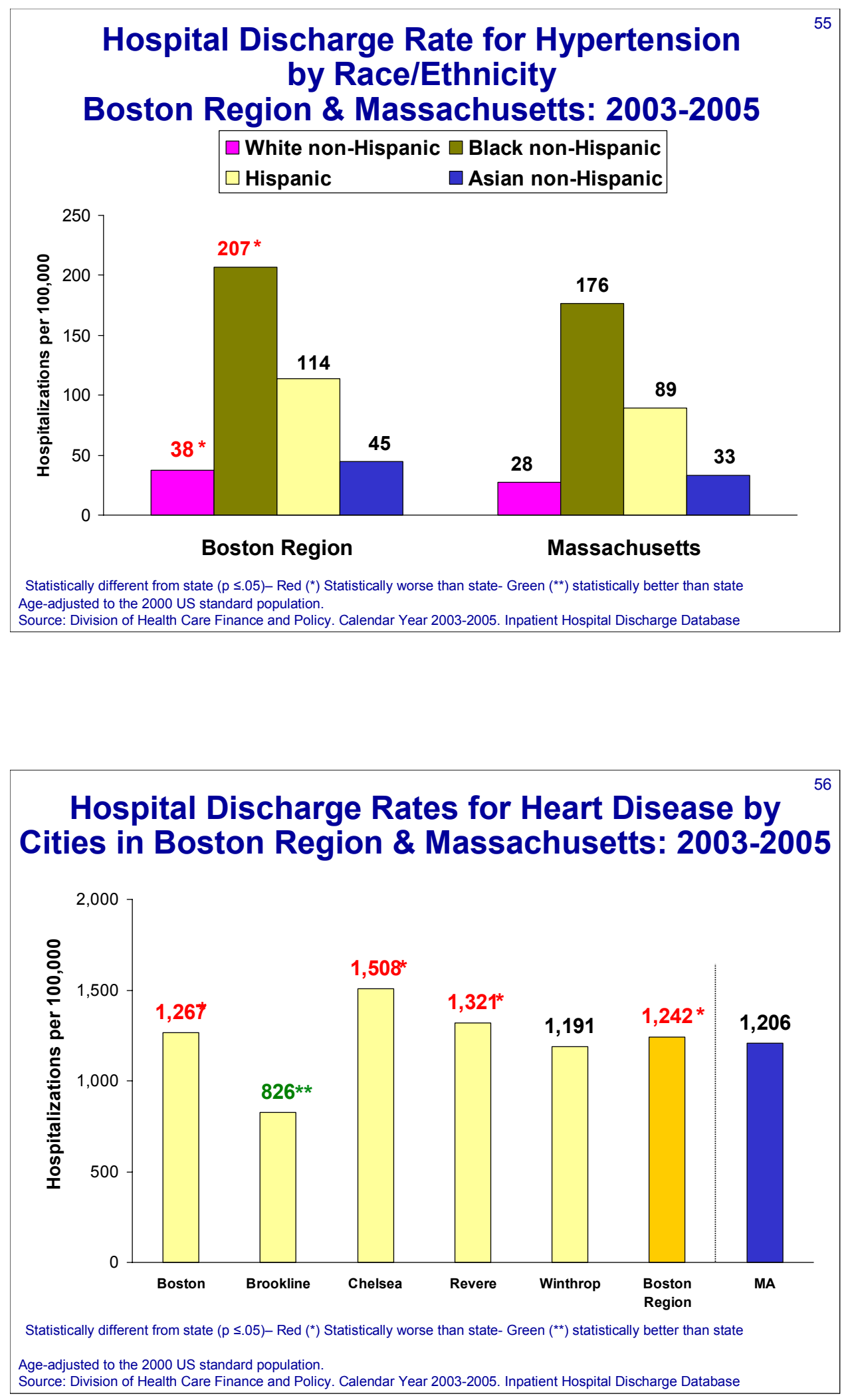


\section{Heart Disease Mortality Rates by EOHHS Region, Massachusetts: 2003-2005}

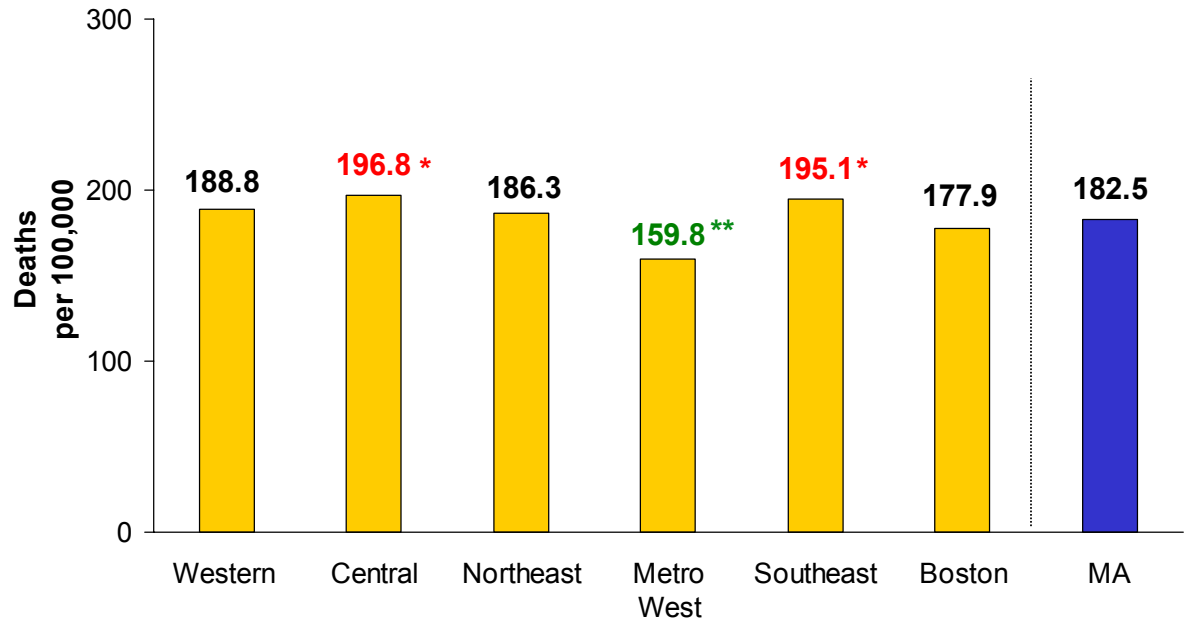

Statistically different from state $(p \leq .05)-\operatorname{Red}\left({ }^{*}\right)$ Statistically worse than state- Green $\left(^{* \star}\right)$ statistically better than state

Heart Disease Mortality Rate by

Cities in Boston Region \& Massachusetts: 2003-2005

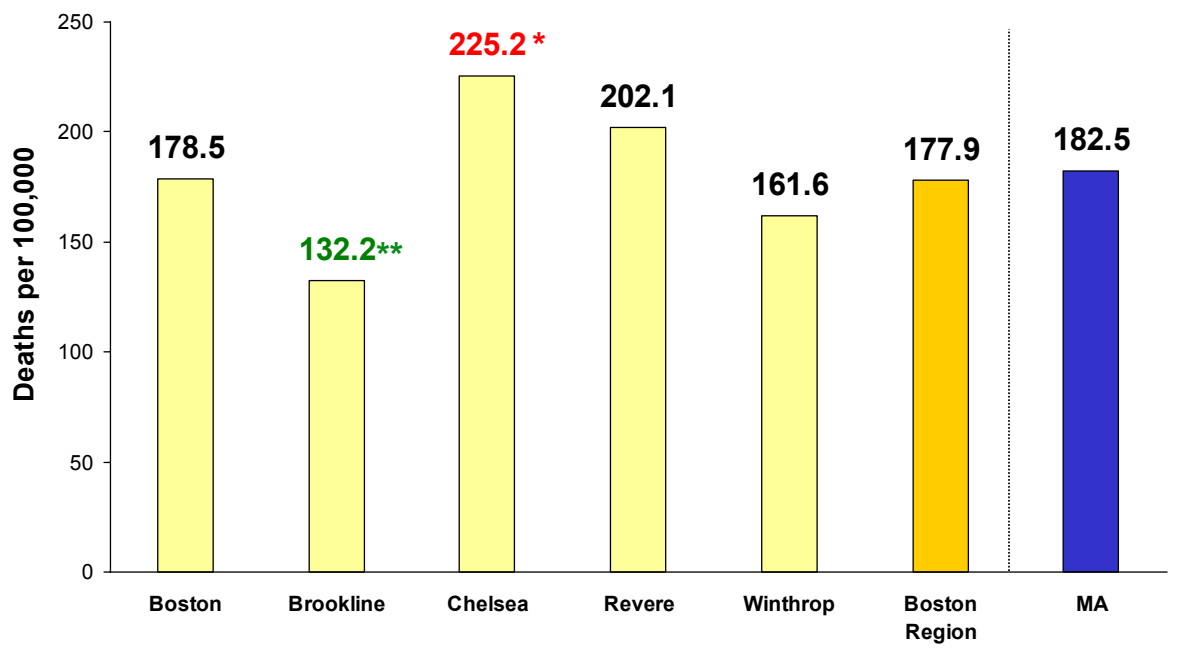

Statistically different from state $(p \leq .05)-$ Red $\left(^{*}\right)$ Statistically worse than state- Green $\left(^{\star *}\right)$ statistically better than state Age-adjusted to the 2000 US standard population

Source: MDPH, Health Information, Statistics, Research and Evaluation Bureau, Research \& Epidemiology Program 
Heart Disease Death Rate by Race/Ethnicity Boston Region and Massachusetts: 2003-2005
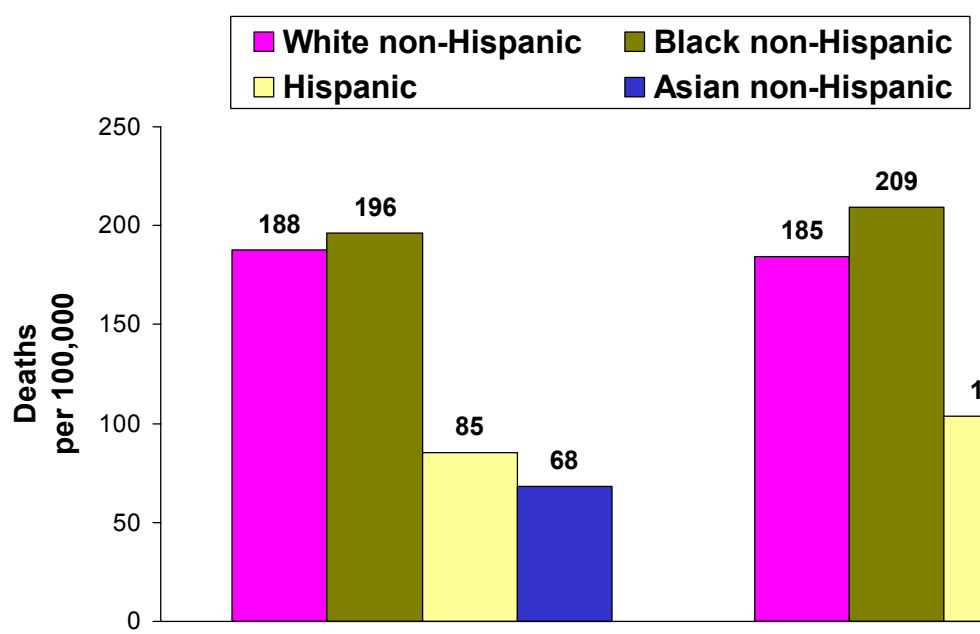

Boston Region

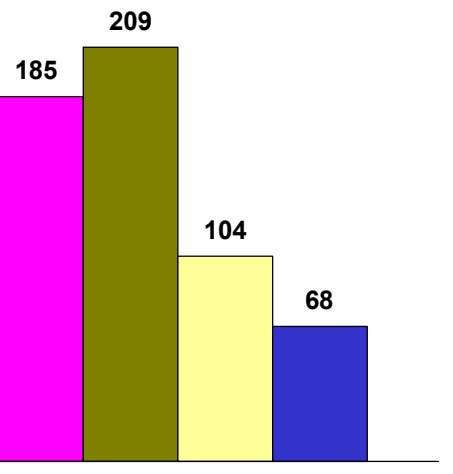

Massachusetts

Statistically different from state $(p \leq .05)-\operatorname{Red}\left({ }^{*}\right)$ Statistically worse than state- Green $\left(^{* *}\right)$ statistically better than state Age-adjusted to the 2000 US standard population

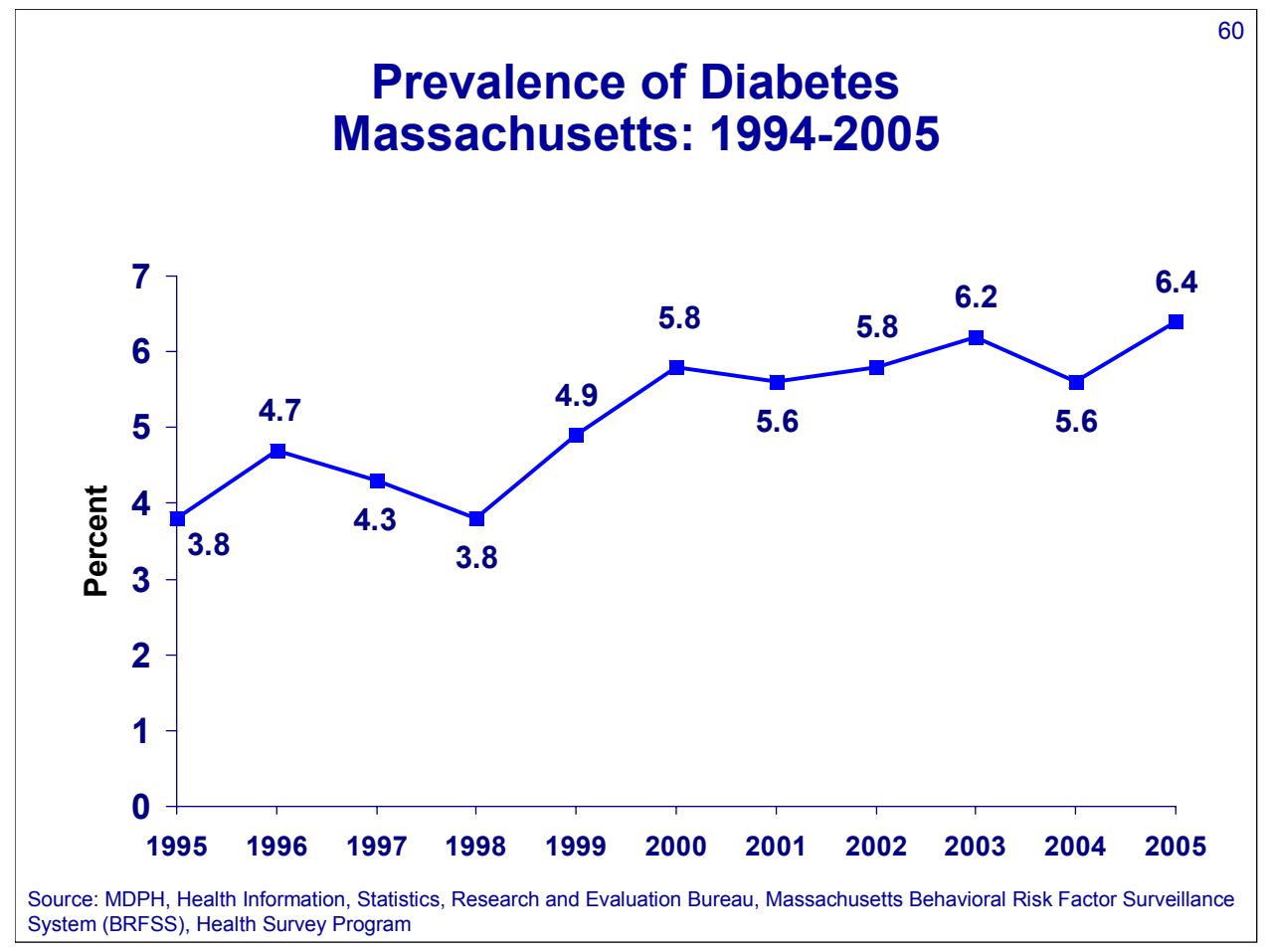




\section{Diabetes Hospital Discharges by EOHHS Region, Massachusetts: 2003-2005}

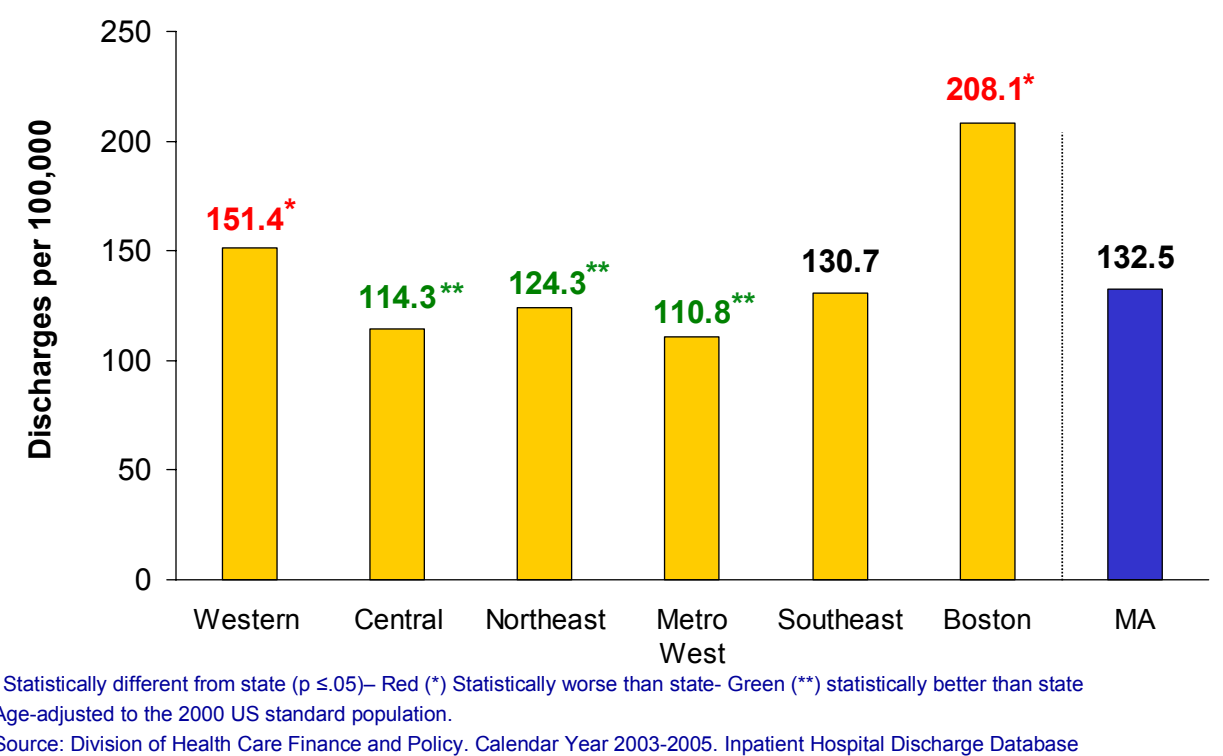

\section{Diabetes Hospital Discharges by}

Cities in Boston Region \& Massachusetts: 2003-2005

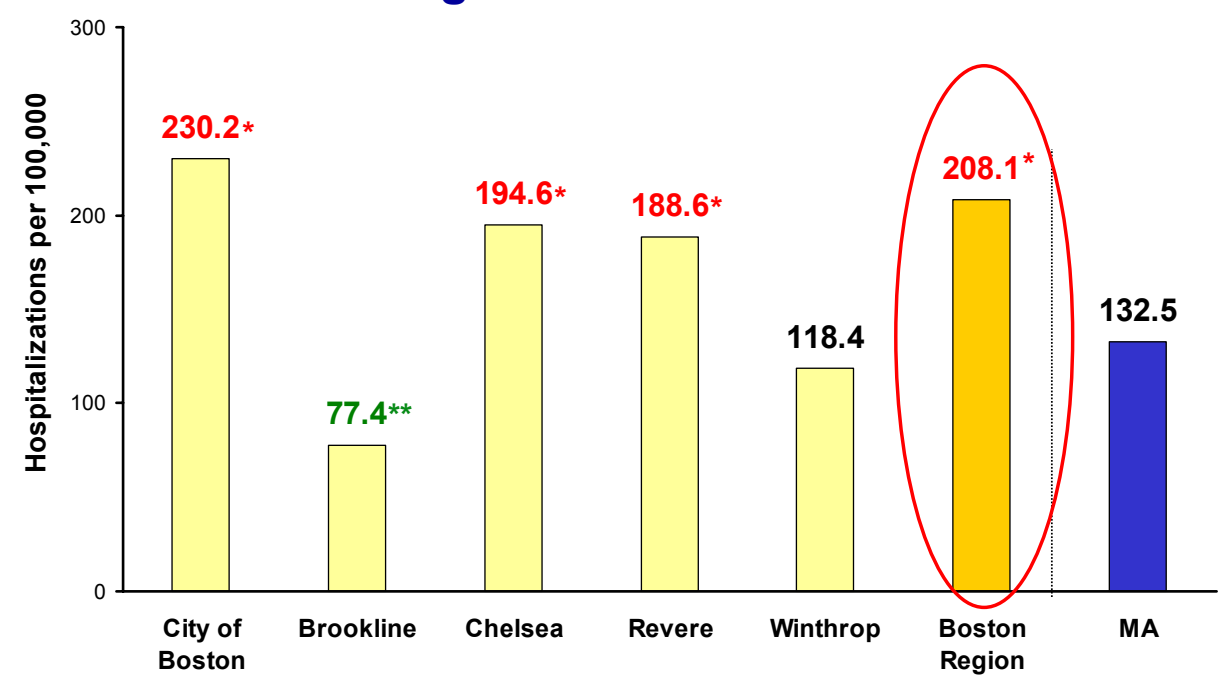

Statistically different from state $(p \leq .05)-\operatorname{Red}\left({ }^{*}\right)$ Statistically worse than state- Green $\left(^{* *}\right)$ statistically better than state Age-adjusted to the 2000 US standard population.

Source: Division of Health Care Finance and Policy. Calendar Year 2003-2005. Inpatient Hospital Discharge Database 


\section{Diabetes Hospital Discharge Rate by Race/Ethnicity Boston Region Massachusetts: 2003-2005}

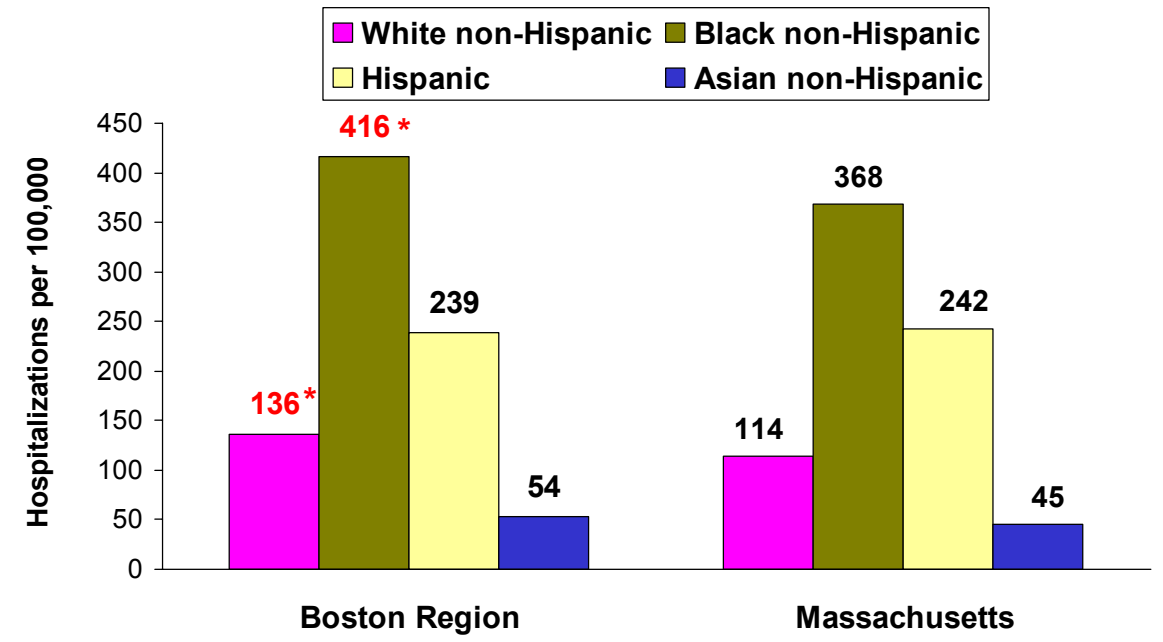

Statistically different from state $(p \leq .05)-\operatorname{Red}\left({ }^{*}\right)$ Statistically worse than state- Green $\left(^{* *}\right)$ statistically better than state Age-adjusted to the 2000 US standard population.

\section{Diabetes Mortality Rates by EOHHS Region} Massachusetts: 2003-2005

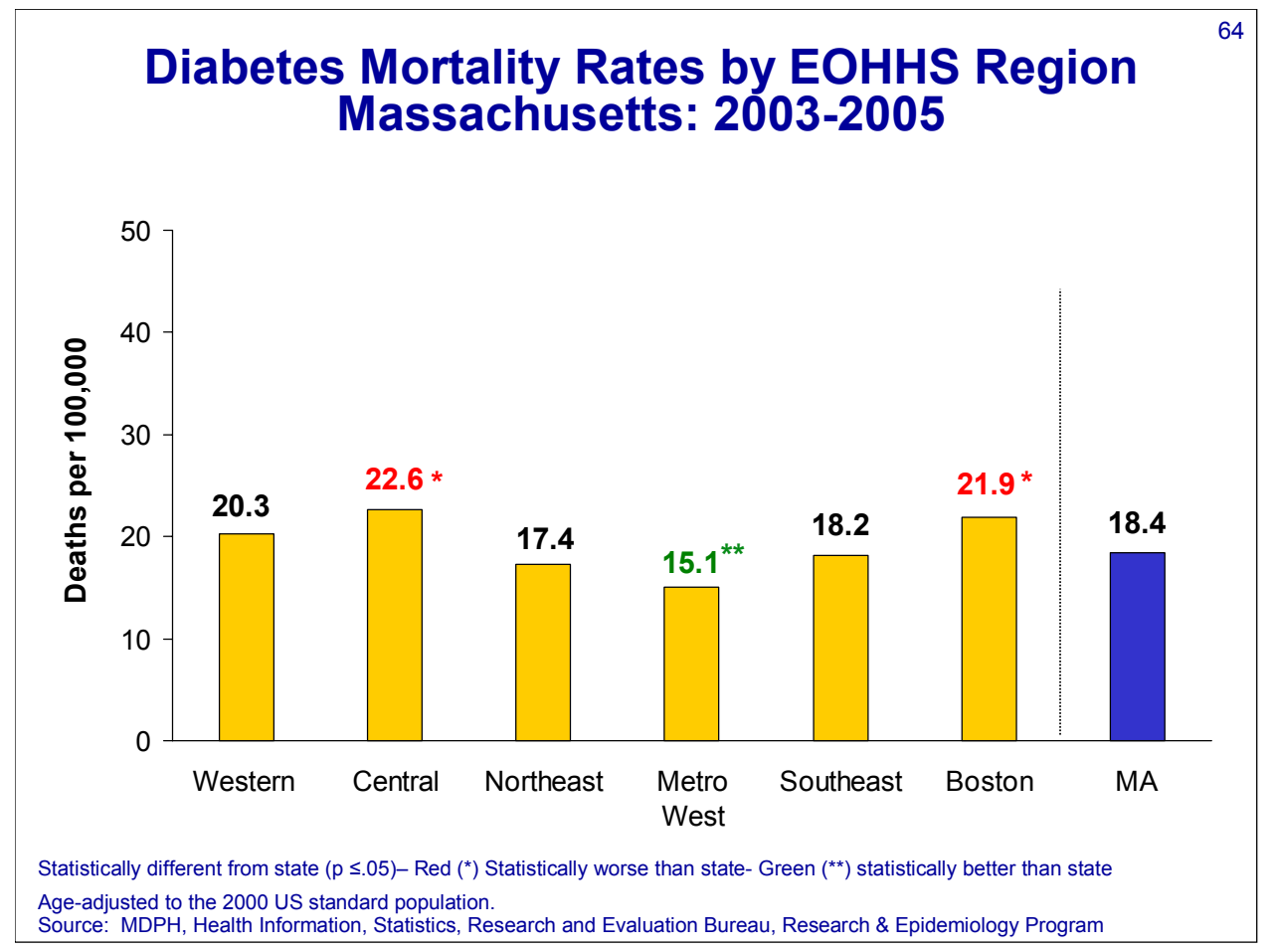



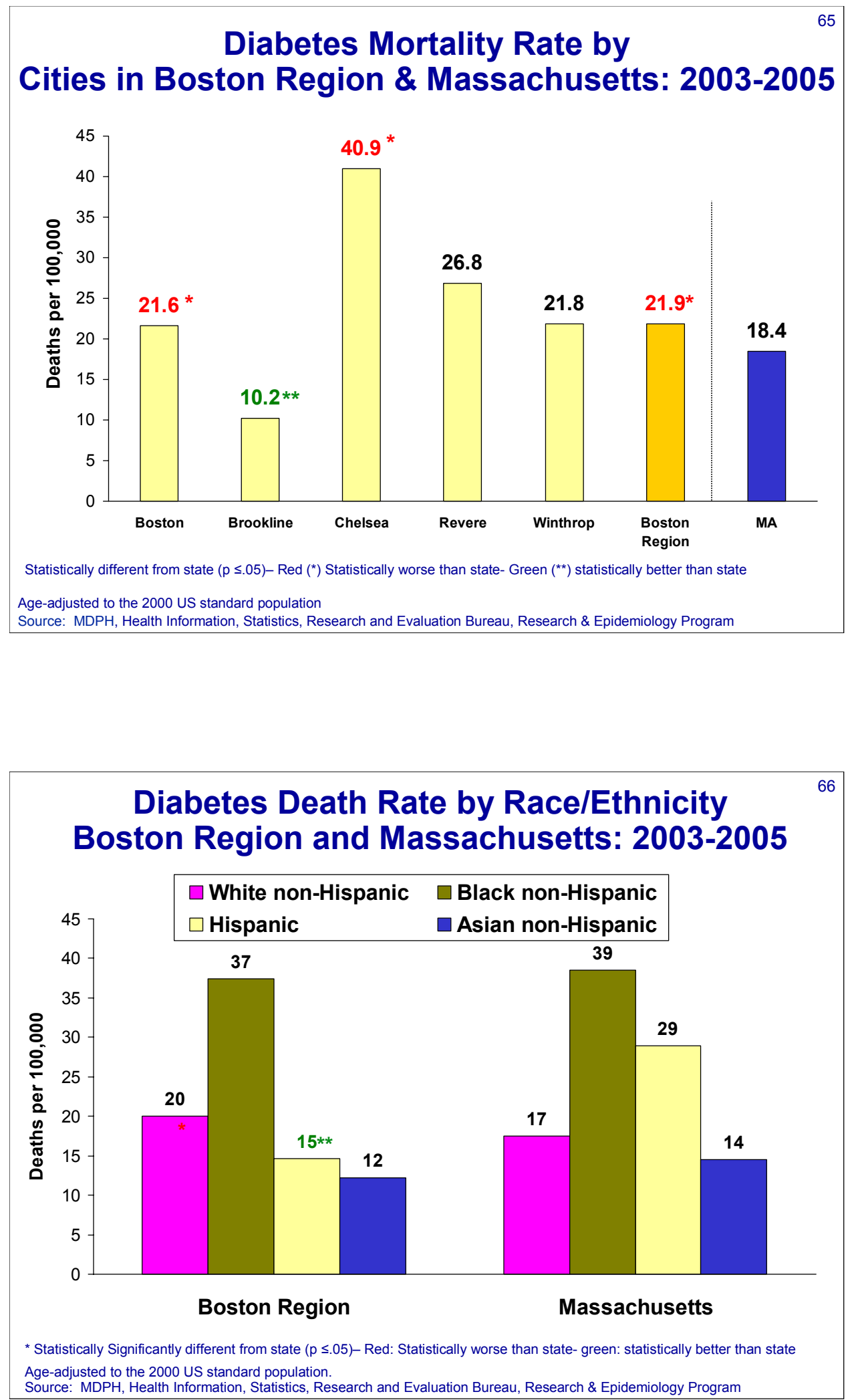


\section{Summary Indicators for Overweight/Obesity by EOHHS Region, Massachusetts:2005}

\begin{tabular}{|c|c|c|c|c|c|c|c|}
\hline Indicator & $\begin{array}{c}\text { MA } \\
\text { Total }\end{array}$ & Western & Central & Northeast & $\begin{array}{l}\text { Metro } \\
\text { West }\end{array}$ & $\begin{array}{c}\text { South } \\
\text { East }\end{array}$ & Boston \\
\hline $\begin{array}{c}\% \text { Obese } \\
\text { Adults }\end{array}$ & 20.7 & 23.3 & 21.7 & 19.2 & 16.8 & 23.6 & 20.9 \\
\hline $\begin{array}{l}\% \text { Adults } \\
\text { Participation in } \\
\text { regular PA }\end{array}$ & 52.6 & 56.4 & 51.5 & 50.6 & 54.7 & 51.8 & 49.5 \\
\hline $\begin{array}{c}\% \text { Adults with } \\
\text { 5+ servings } \\
\text { fruits/ } \\
\text { vegetables }\end{array}$ & 28.6 & 28.4 & 29.3 & 26.4 & 33.0 & 26.4 & 26.4 \\
\hline $\begin{array}{c}\text { \% Adults who } \\
\text { had } \\
\text { cholesterol } \\
\text { checked }\end{array}$ & 79.3 & 74.0 & 76.6 & 79.2 & 84.8 & 82.0 & 73.3 \\
\hline $\begin{array}{l}\text { \% Adults with } \\
\text { high } \\
\text { cholesterol }\end{array}$ & 35.7 & 34.2 & 35.9 & 34.5 & 38.4 & 36.5 & 31.2 \\
\hline
\end{tabular}

\section{Summary Indicators for Chronic Disease Outcomes by EOHHS Region}

\begin{tabular}{||c|c|c|c|c|c|c|c|}
\hline Indicator & $\begin{array}{c}\text { MA } \\
\text { Total }\end{array}$ & Western & Central & Northeast & $\begin{array}{c}\text { Metro } \\
\text { West }\end{array}$ & $\begin{array}{c}\text { South } \\
\text { East }\end{array}$ & Boston \\
\hline \% Adults with HBP & $\mathbf{2 5 . 3}$ & 26.3 & 25.3 & 22.8 & 25.1 & 28.9 & 21.9 \\
\hline $\begin{array}{c}\text { \% Adults take HBP } \\
\text { medications }\end{array}$ & $\mathbf{7 5 . 3}$ & 76.0 & 74.0 & 78.6 & 73.5 & 76.5 & 71.3 \\
\hline $\begin{array}{c}\text { Hypertension } \\
\text { Hospital } \\
\text { Discharges } \\
\text { (2003-2005) }\end{array}$ & $\mathbf{3 8 . 7}$ & 35.0 & 30.9 & 32.9 & 31.0 & 38.7 & 84.6 \\
\hline $\begin{array}{c}\text { Heart Disease } \\
\text { Death Rate } \\
\text { (2003-2005) }\end{array}$ & 182.5 & 188.8 & 196.8 & 186.3 & 159.8 & 195.1 & 177.9 \\
\hline $\begin{array}{c}\text { Diabetes Hospital } \\
\text { Discharges } \\
\text { (2003-2005) }\end{array}$ & $\mathbf{1 3 2 . 5}$ & 151.4 & 114.3 & 124.3 & 110.8 & 130.7 & 208.1 \\
\hline $\begin{array}{c}\text { Diabetes Death } \\
\text { Rate (2003-2005) }\end{array}$ & $\mathbf{1 8 . 4}$ & 20.3 & 22.6 & 17.3 & 15.1 & 18.2 & 21.9 \\
\hline
\end{tabular}

Better Outcome (significantly different from state) $\square$ Worse Outcome (significantly different from state) 


\section{CANCER}


Adults Ages 50+ who have had a Sigmoidoscopy or Colonoscopy in Past 5 Years by EOHHS Region Massachusetts: 2005

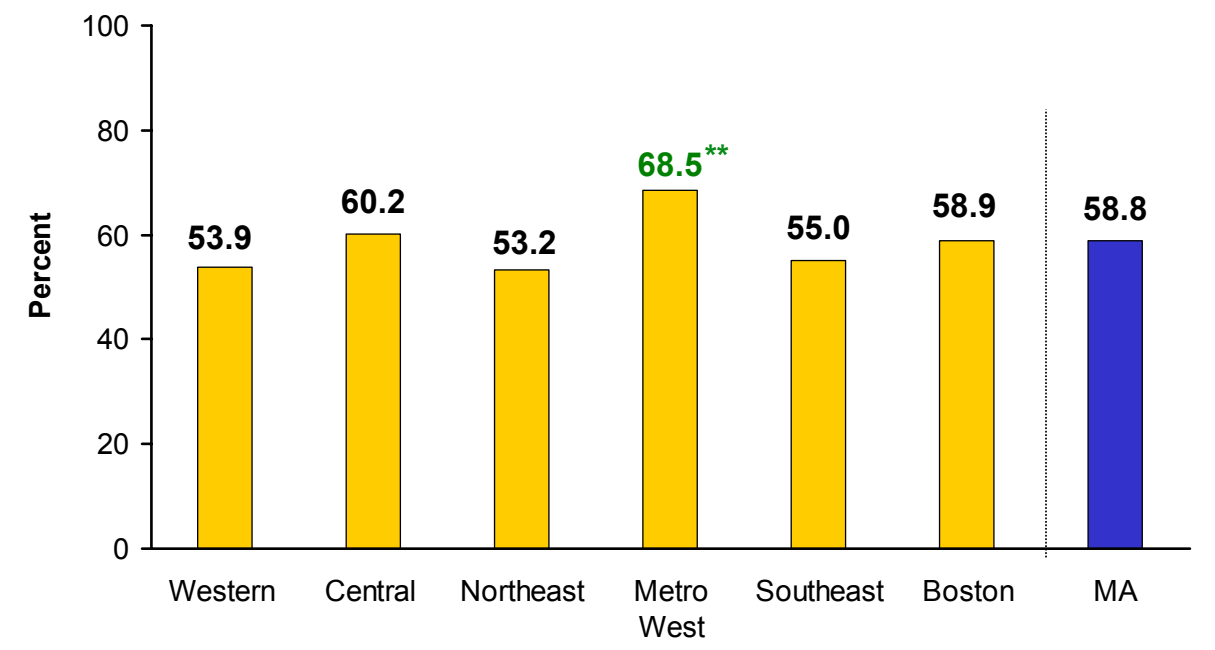

Statistically different from state $(p \leq .05)-\operatorname{Red}\left({ }^{*}\right)$ Statistically worse than state- Green $\left(^{* \star}\right)$ statistically better than state

Source: MDPH, Health Information, Statistics, Research and Evaluation Bureau, Health Survey Program

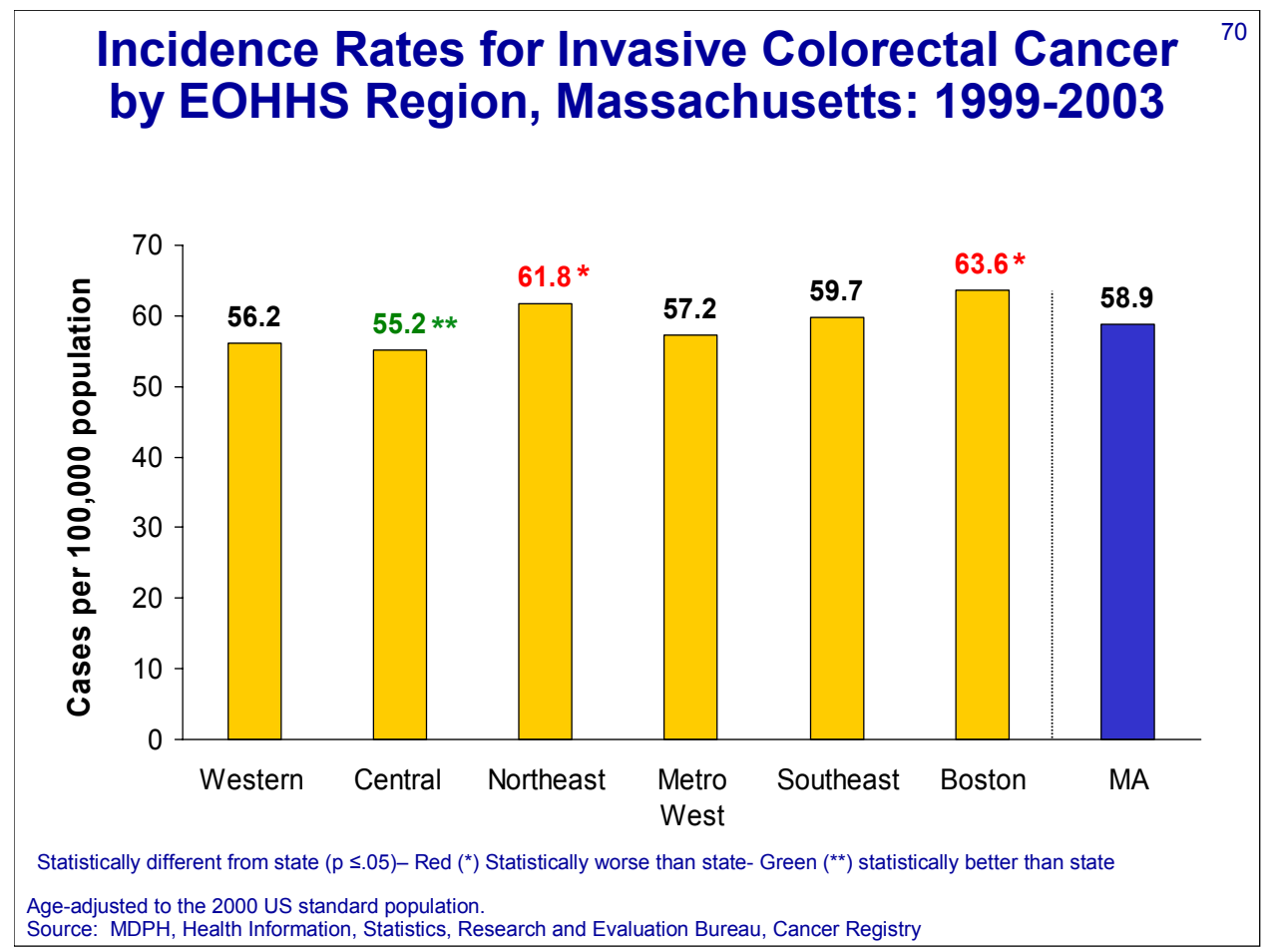




\section{Incidence Rates for Early Stage Colorectal Cancer ${ }^{71}$ MALES \\ by EOHHS Region, Massachusetts: 1999-2003}

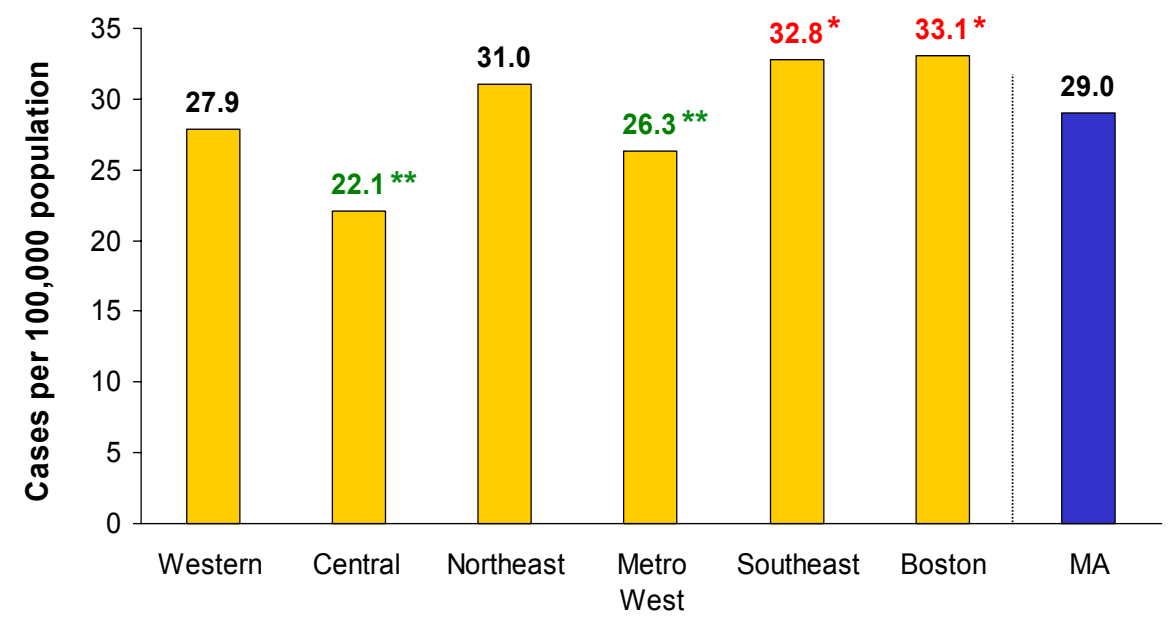

Statistically different from state $(p \leq .05)-\operatorname{Red}\left({ }^{*}\right)$ Statistically worse than state- Green $\left(^{* *}\right)$ statistically better than state

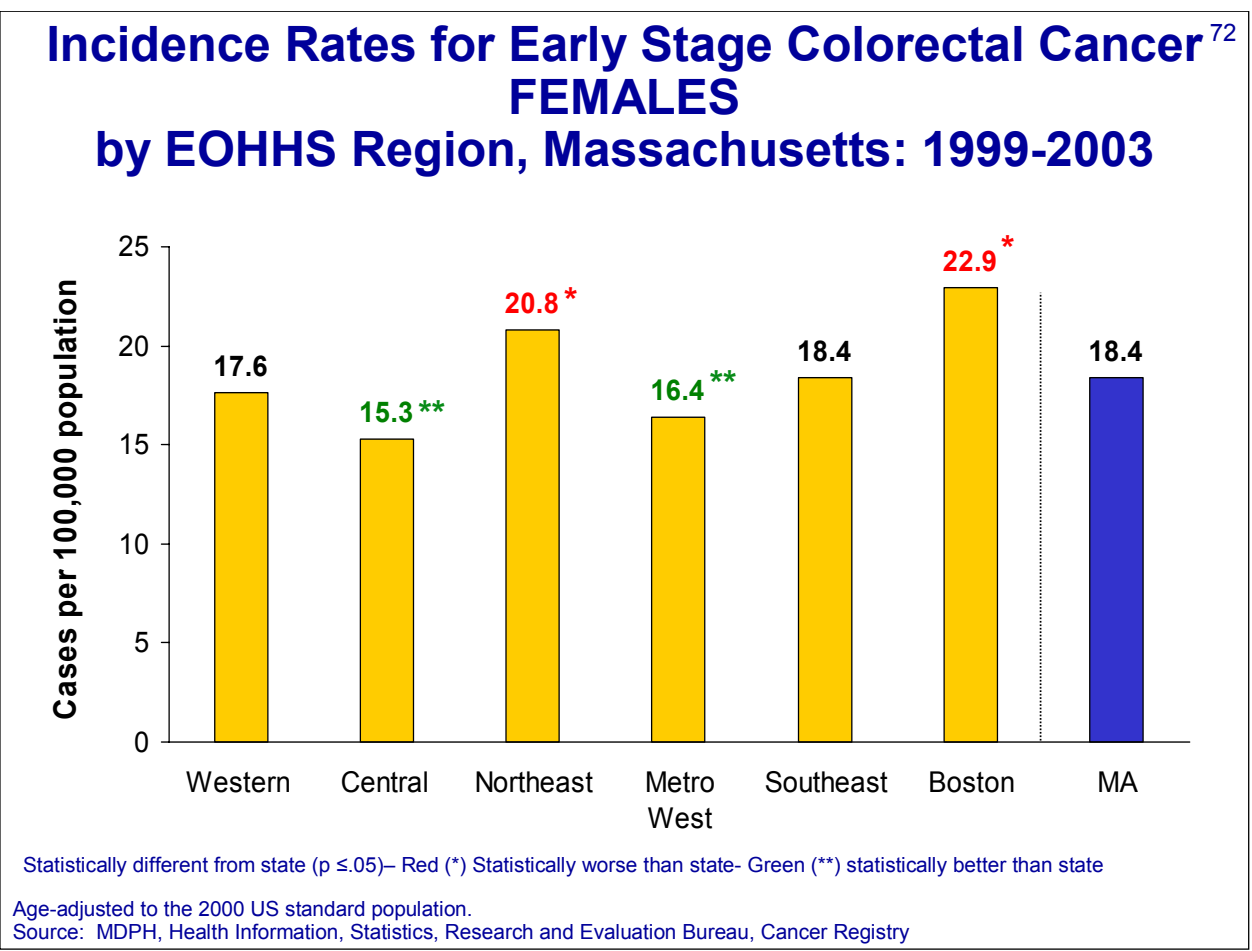




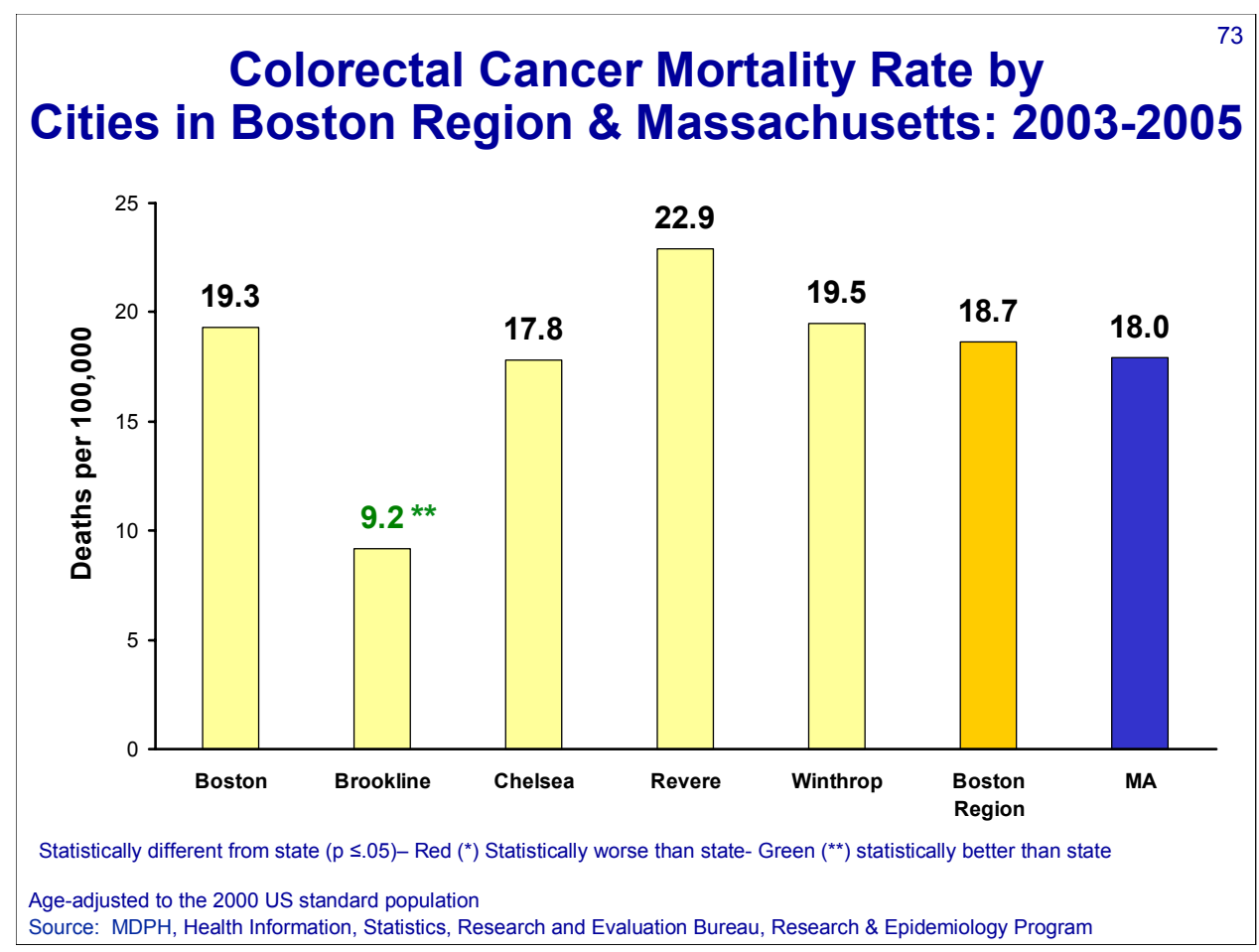

Men Ages 50 and Older who had a Prostate-Specific Antigen Test in the Past Two Years by EOHHS Region, Massachusetts: 2004

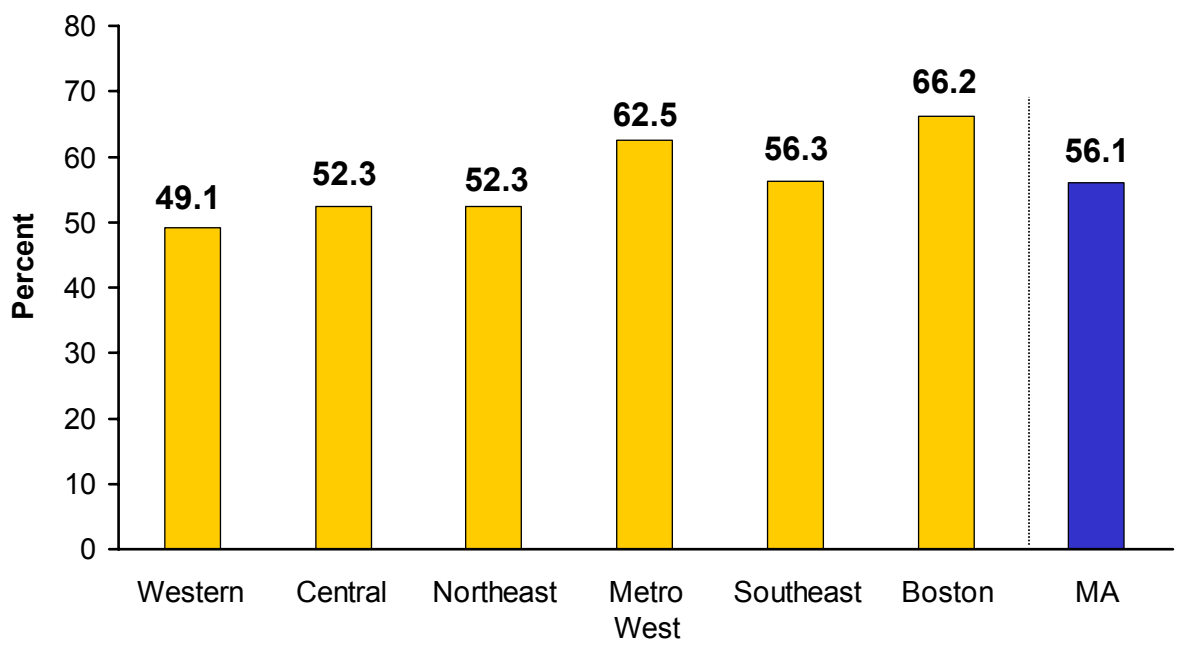

Statistically different from state $(p \leq .05)-\operatorname{Red}\left({ }^{*}\right)$ Statistically worse than state- Green $\left(^{* \star}\right)$ statistically better than state Source: MDPH, Health Information, Statistics, Research and Evaluation Bureau, Health Survey Program 


\section{Men Ages 50 and Older who had a Digital Rectal Exam in the Past Two Years by EOHHS Region Massachusetts: 2004}

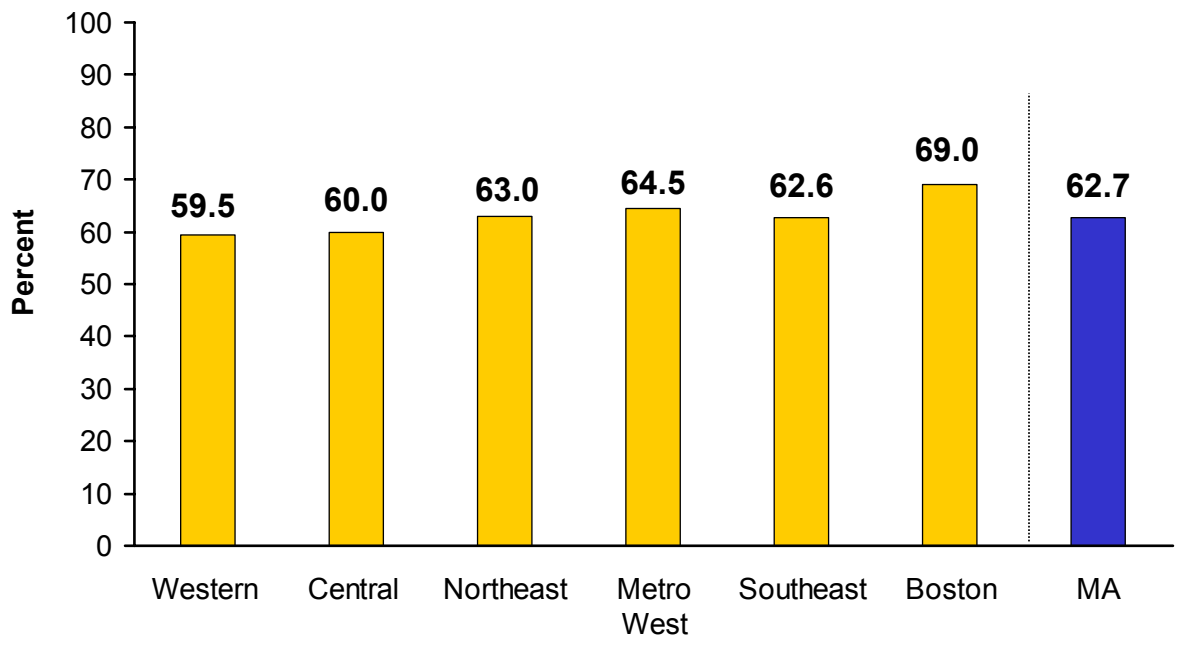

Statistically different from state $(p \leq .05)-\operatorname{Red}\left({ }^{*}\right)$ Statistically worse than state- Green $\left(^{* *}\right)$ statistically better than state Source: MDPH, Health Information, Statistics, Research and Evaluation Bureau, Health Survey Program

\section{Adults Ages 50 and Older who had a Blood Stool Test in the Past Two Years by EOHHS Region Massachusetts: 2005}

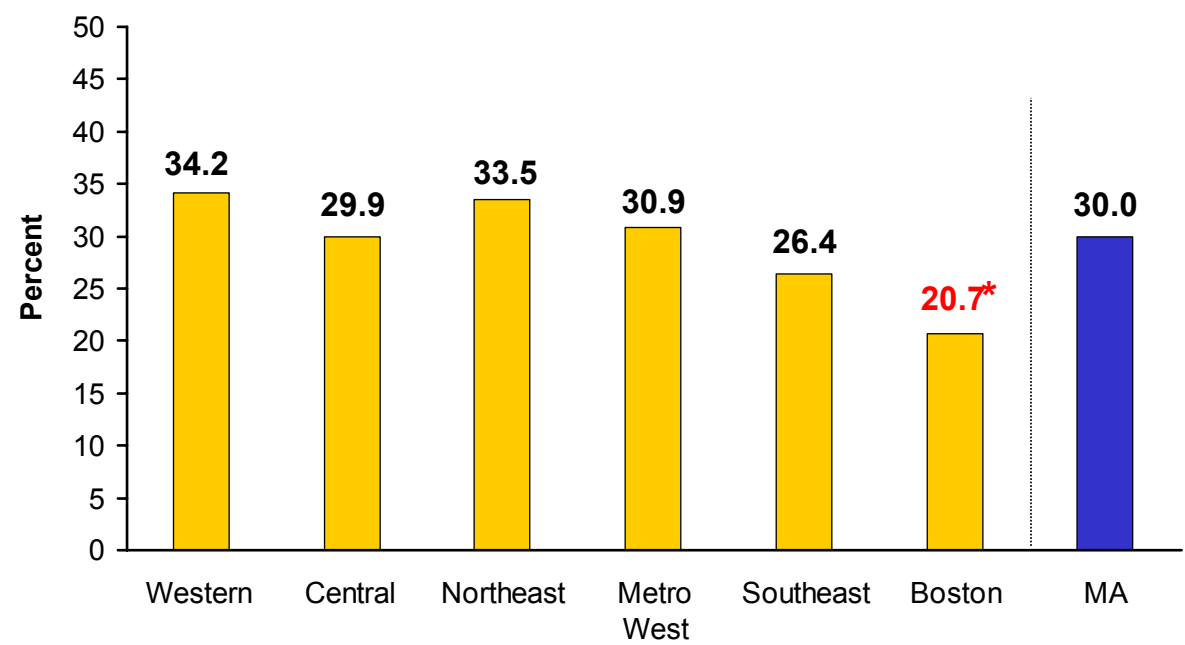

Statistically different from state $(p \leq .05)-\operatorname{Red}\left({ }^{*}\right)$ Statistically worse than state- Green $\left.{ }^{* *}\right)$ statistically better than state Source: MDPH, Health Information, Statistics, Research and Evaluation Bureau, Health Survey Program 
Incidence Rates for Invasive Prostate Cancer by EOHHS Region, Massachusetts: 1999-2003

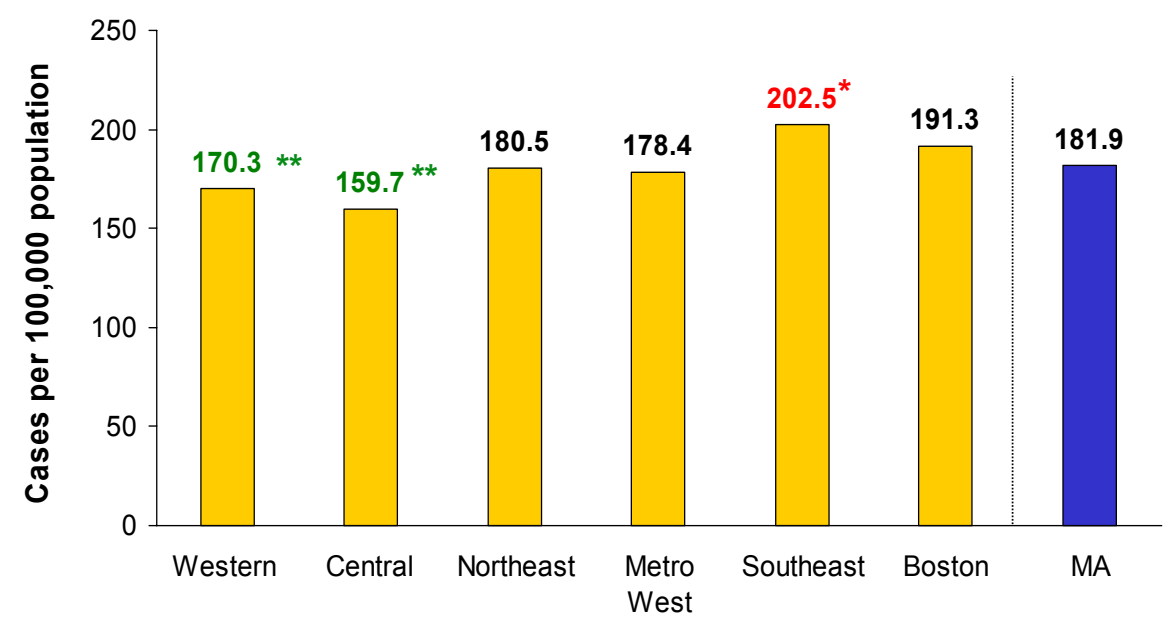

Statistically different from state $(p \leq .05)-\operatorname{Red}\left({ }^{*}\right)$ Statistically worse than state- Green $\left(^{* \star}\right)$ statistically better than state

\section{Prostate Cancer Incidence Rates by}

\section{Cities in Boston Region \& Massachusetts: 1999-2003}

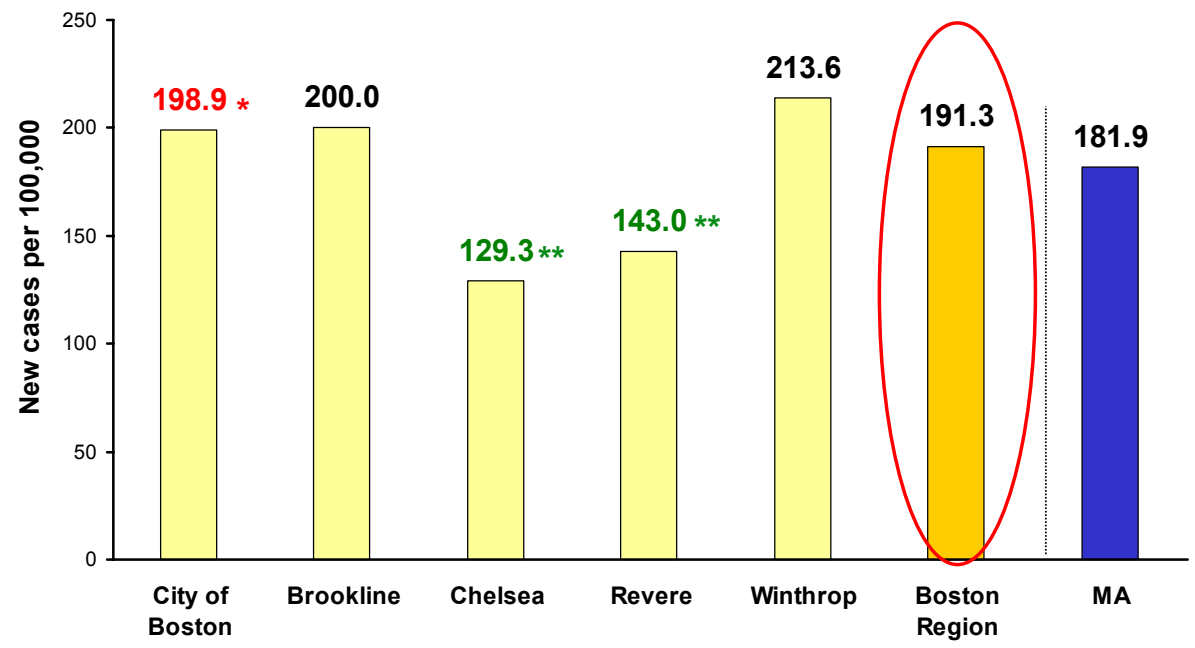

Statistically different from state $(p \leq .05)-\operatorname{Red}\left({ }^{*}\right)$ Statistically worse than state- Green $\left(^{* *}\right)$ statistically better than state 
Incidence Rates for Early Stage Prostate Cancer by EOHHS Region, Massachusetts: 1999-2003

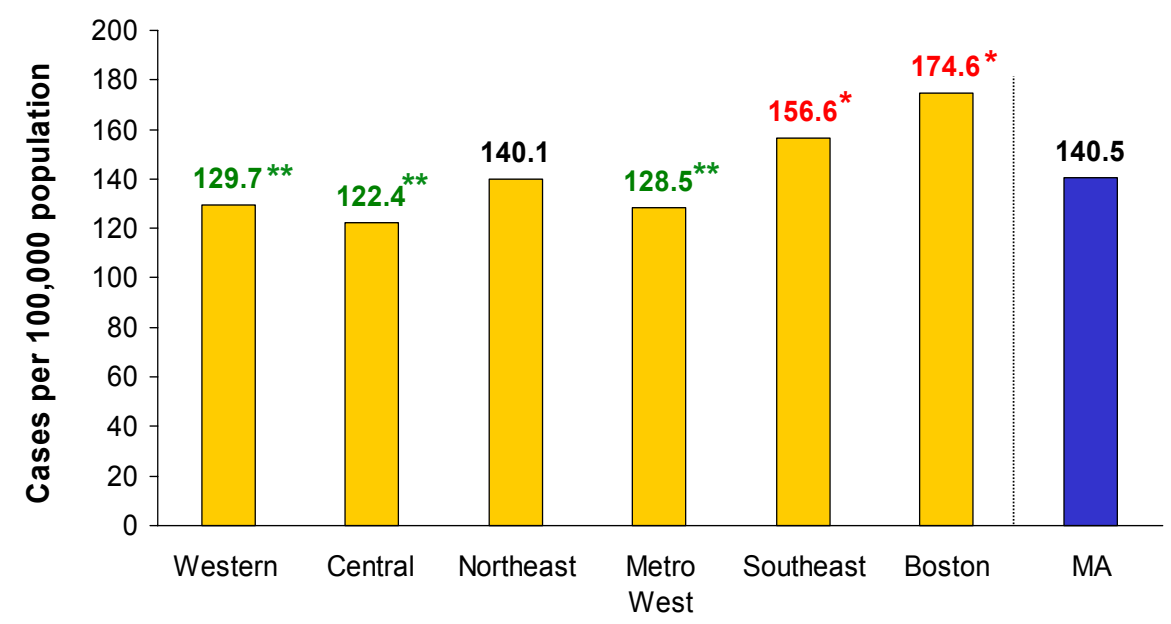

Statistically different from state $(p \leq .05)-$ Red $\left({ }^{*}\right)$ Statistically worse than state- Green $\left(^{* *}\right)$ statistically better than state Age-adjusted to the 2000 US standard population.

Source: MDPH, Health Information, Statistics, Research and Evaluation Bureau, Cancer Registry

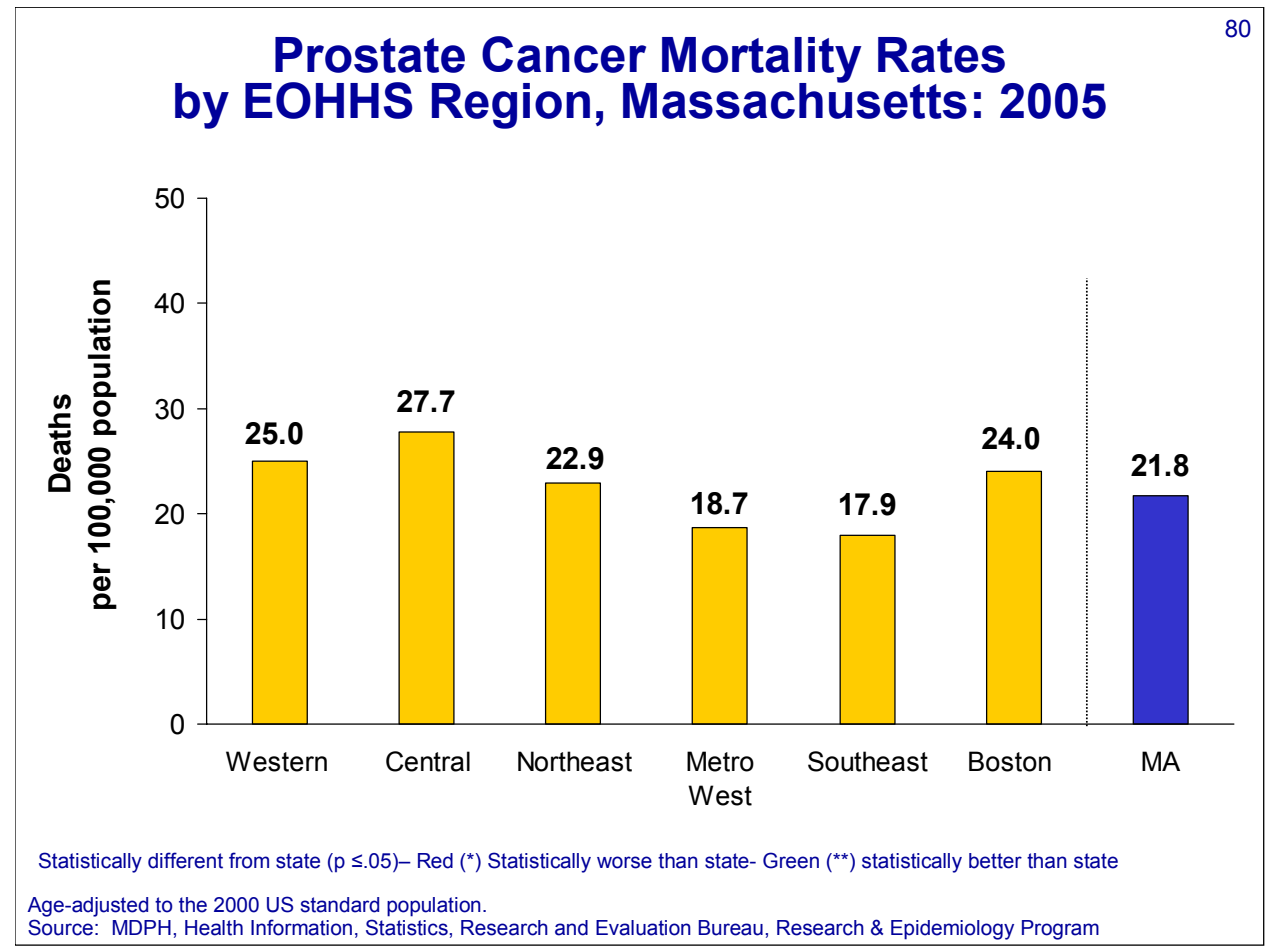


Women who had a Clinical Breast Exam in the Past Two Years by Race and Ethnicity

Boston Region and Massachusetts: 2004

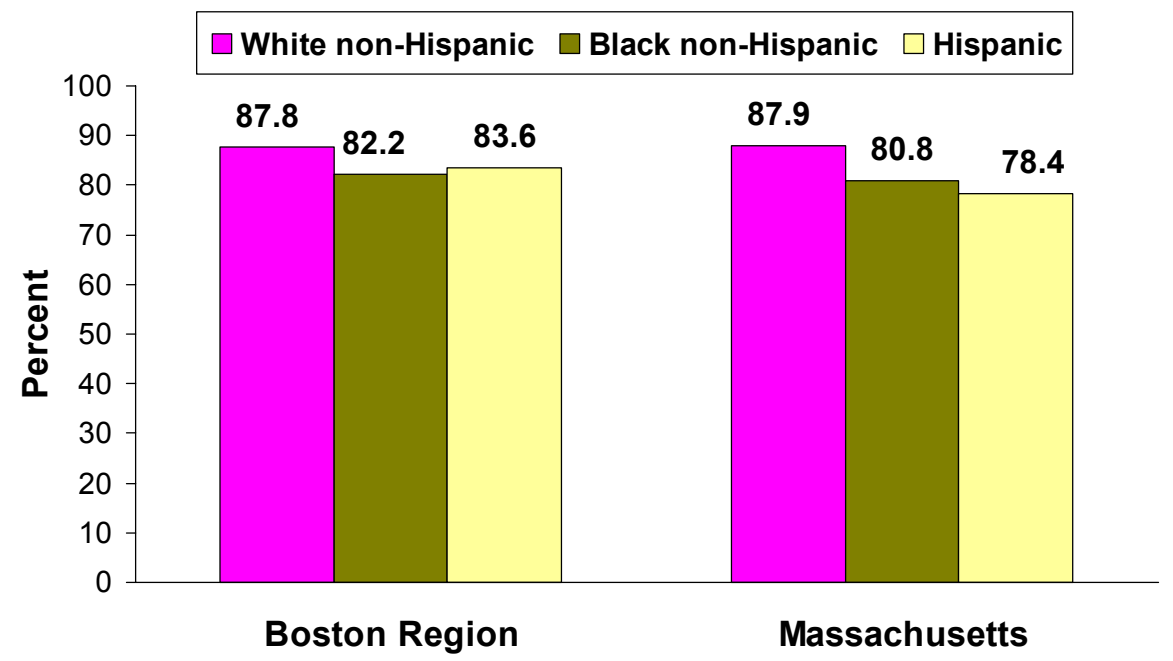

Statistically different from state $(p \leq .05)-\operatorname{Red}\left({ }^{*}\right)$ Statistically worse than state- Green $\left(^{* *}\right)$ statistically better than state Source: MDPH, Health Information, Statistics, Research and Evaluation Bureau, Health Survey Program

\section{Women Ages 40 and Older who had a Clinical Breast Exam in the Past Two Years by EOHHS Region, Massachusetts: 2004}

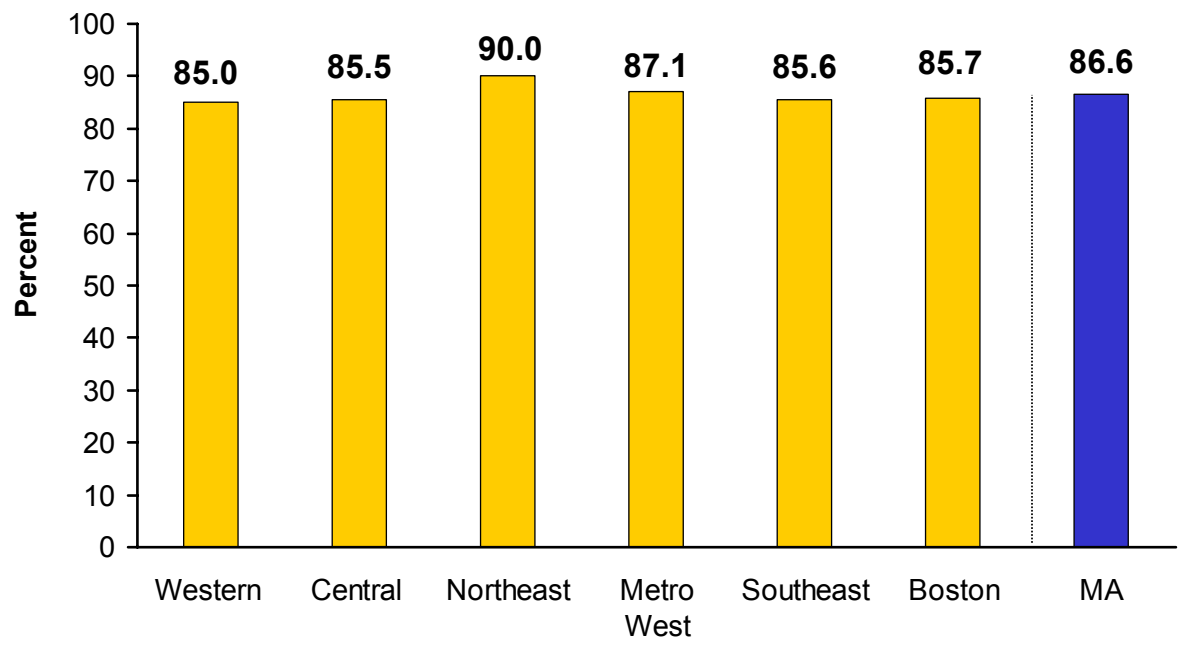


Women Ages 40+ who have had a mammography in Past 2 Years by EOHHS Region Massachusetts: 2005

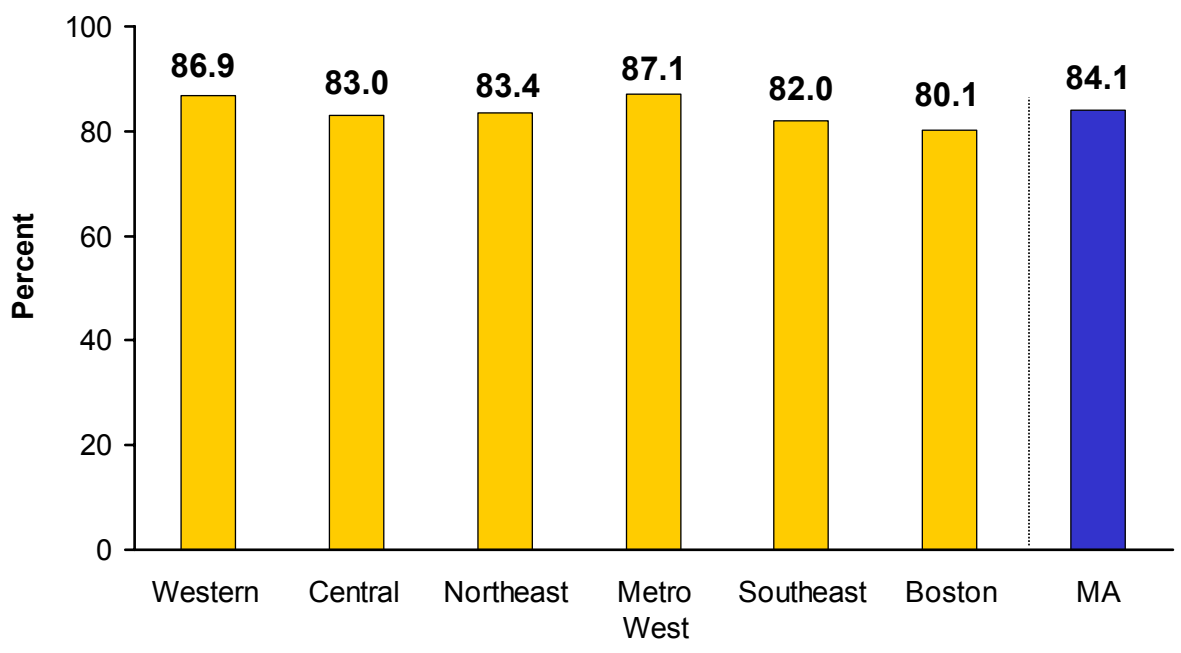

Statistically different from state $(p \leq .05)-\operatorname{Red}\left({ }^{*}\right)$ Statistically worse than state- Green $\left(^{* \star}\right)$ statistically better than state Source: MDPH, Health Information, Statistics, Research and Evaluation Bureau, Health Survey Program

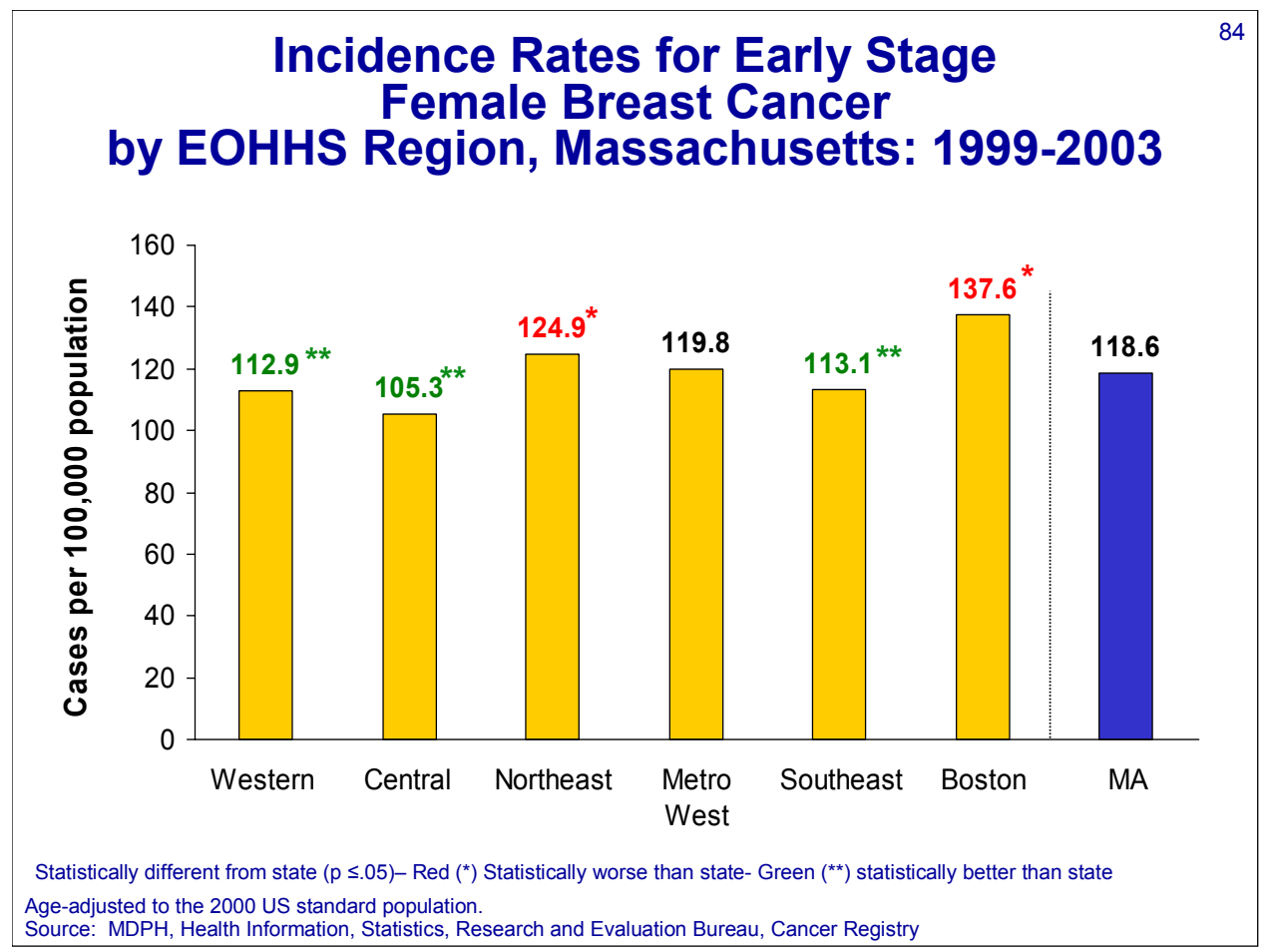



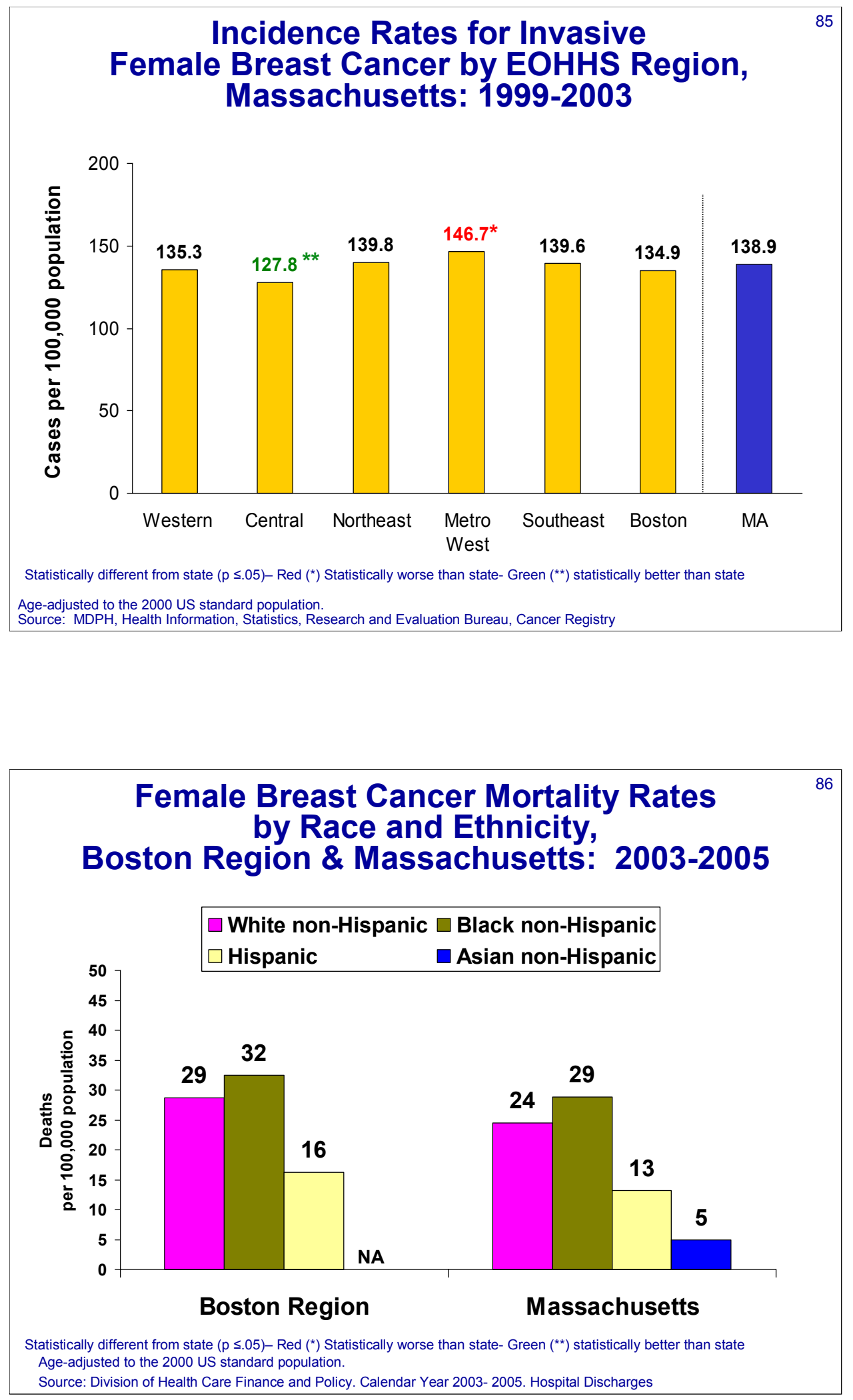

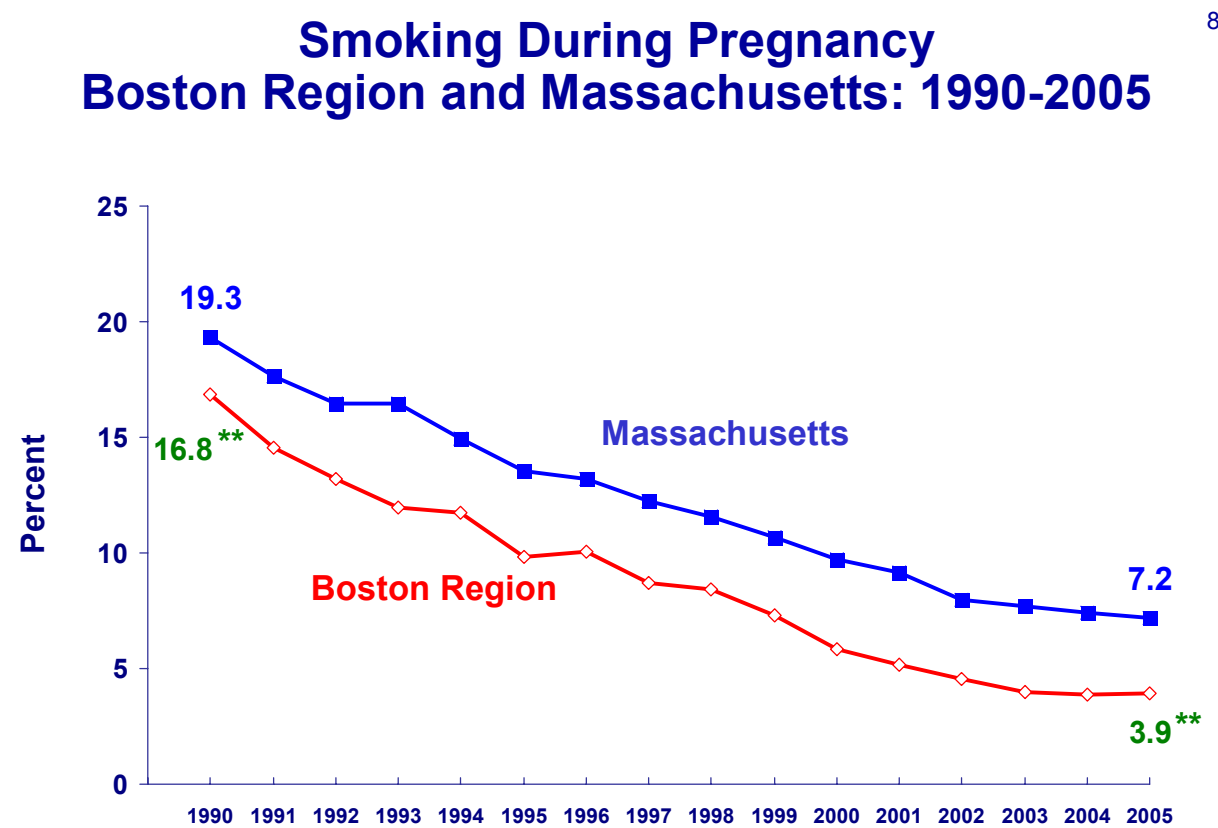

Statistically different from state $(p \leq .05)-\operatorname{Red}\left({ }^{*}\right)$ Statistically worse than state- Green $\left(^{* \star}\right)$ statistically better than state

Source: MDPH, Health Information, Statistics, Research and Evaluation Bureau, Research \& Epidemiology Program

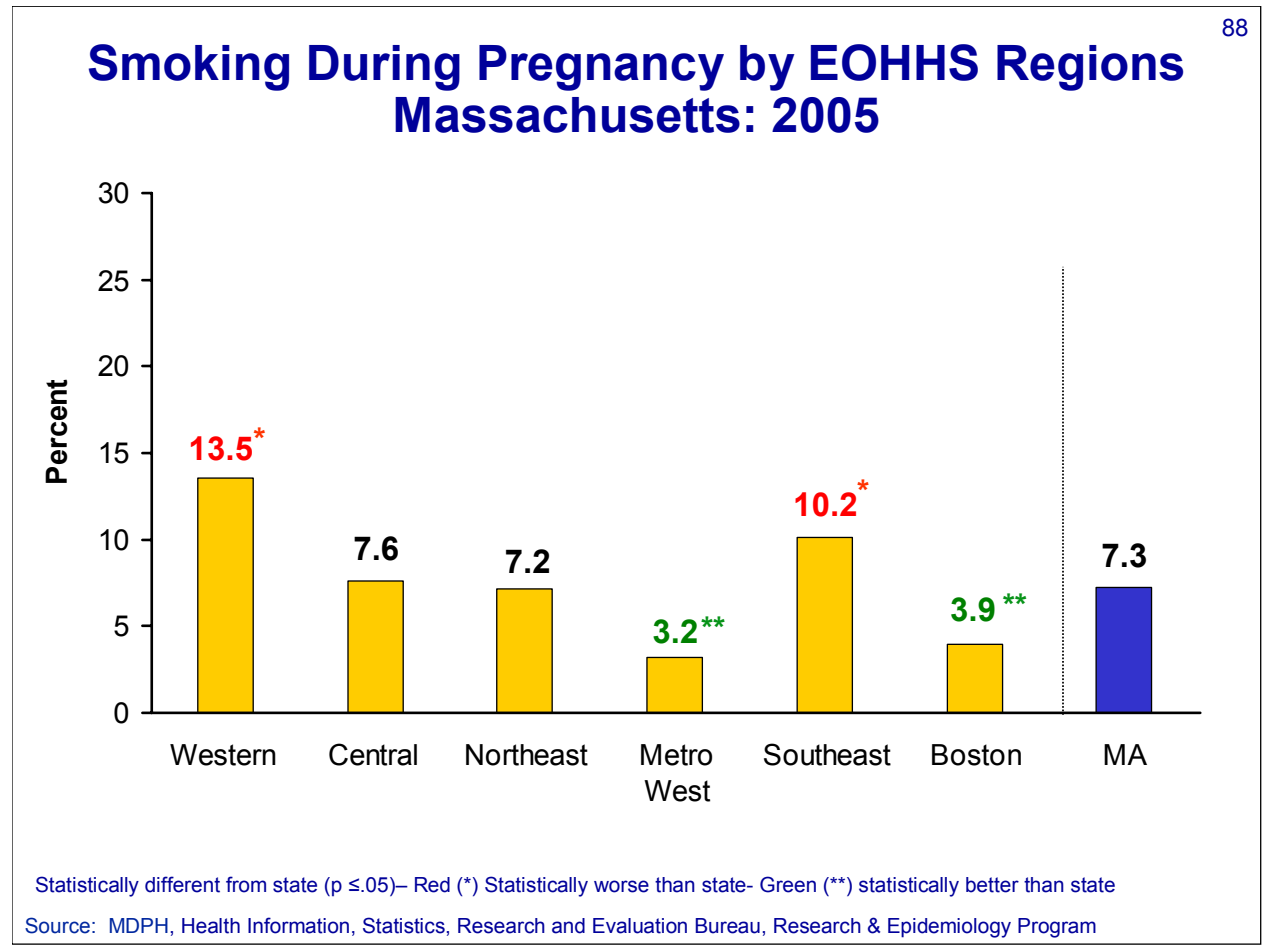


Percent of Mothers Smoking During
Pregnancy for Cities in Boston Region: 2005

\begin{tabular}{|lccc|}
\hline & $\underline{\%}$ & $\underline{\text { Smokers }}$ & $\underline{\text { Births }}$ \\
Massachusetts & $7.2 \%$ & 5,555 & 76,653 \\
Boston Region & $\underline{3.9 \% * *}$ & $\underline{378}$ & $\underline{9,627}$ \\
\hline
\end{tabular}

City of Boston

$3.6 \%$ **

273

7,540

Brookline

$<1 \%$

$<5$

613

Chelsea

$4.8 \%$ **

31

648

Revere

$9.7 \%$

62

641

Winthrop

$4.8 \%$

9

186

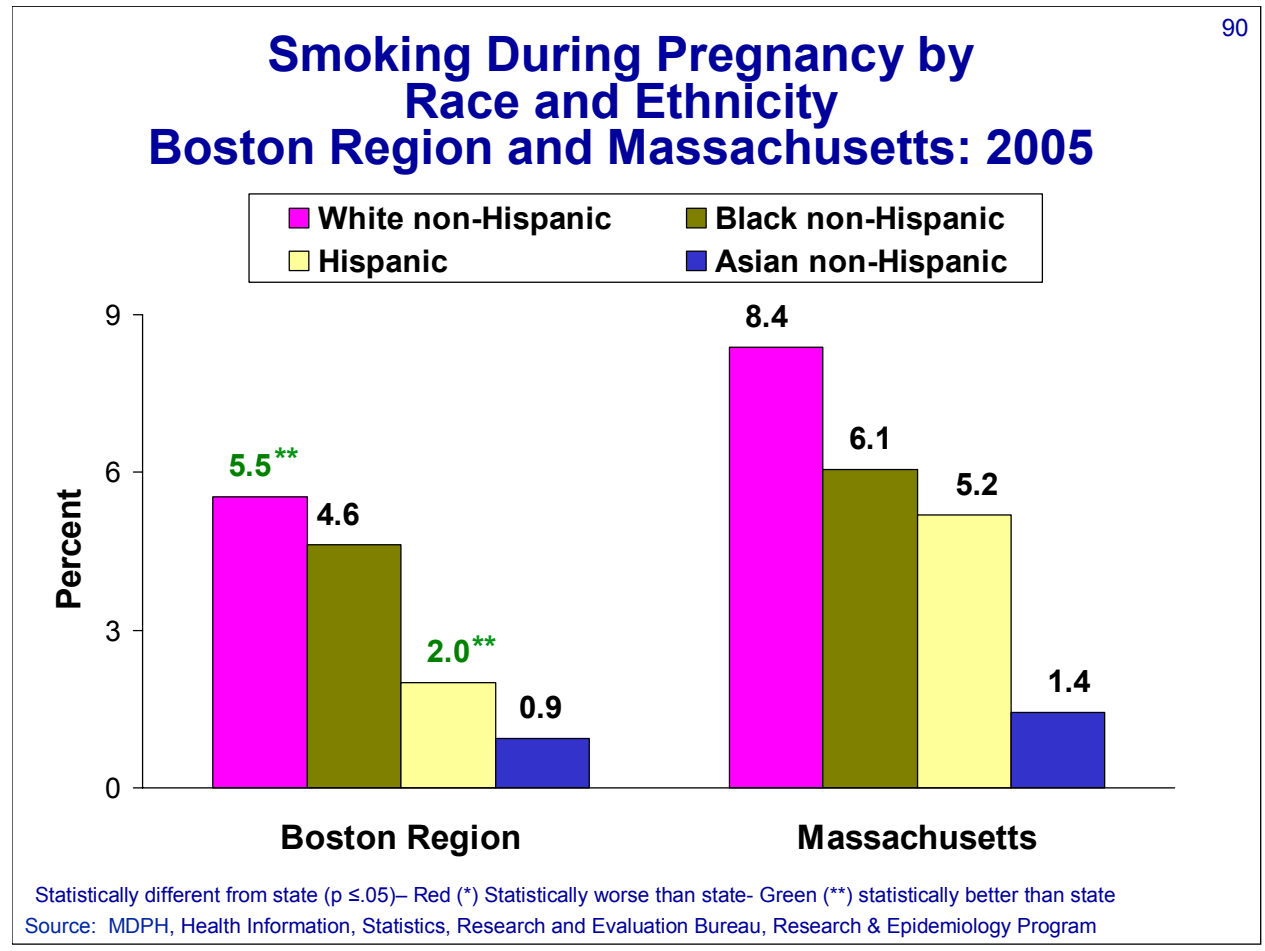


Incidence Rates for Invasive Lung Cancer
by EOHHS Region, Massachusetts: 1999-2003

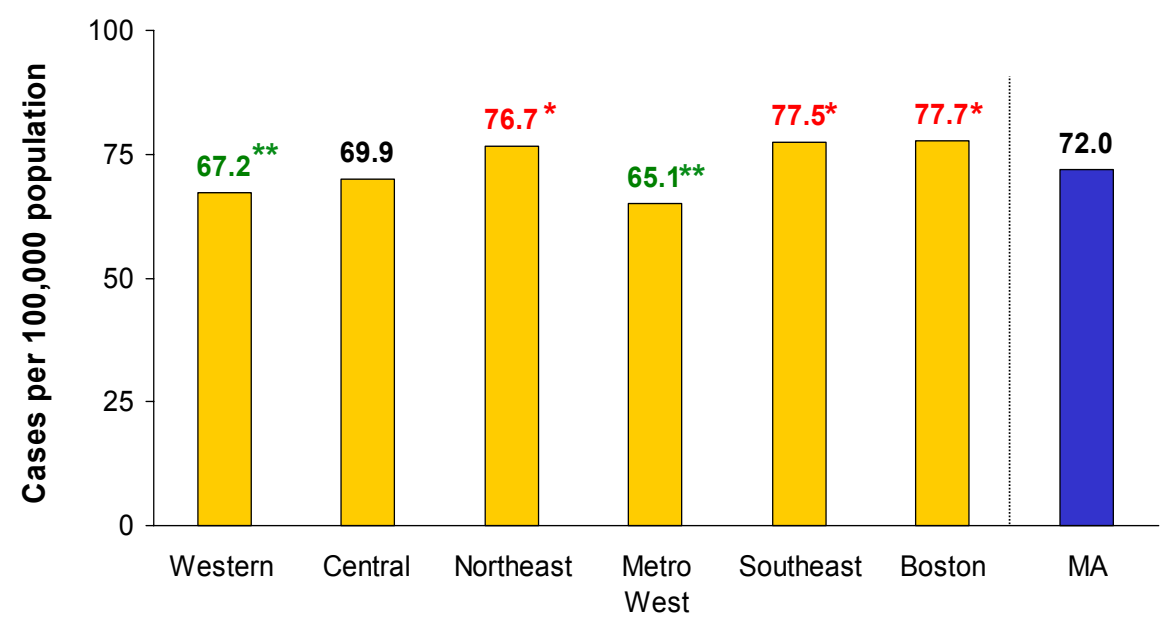

Statistically different from state $(p \leq .05)-\operatorname{Red}\left({ }^{\star}\right)$ Statistically worse than state- Green $\left(^{\star \star}\right)$ statistically better than state

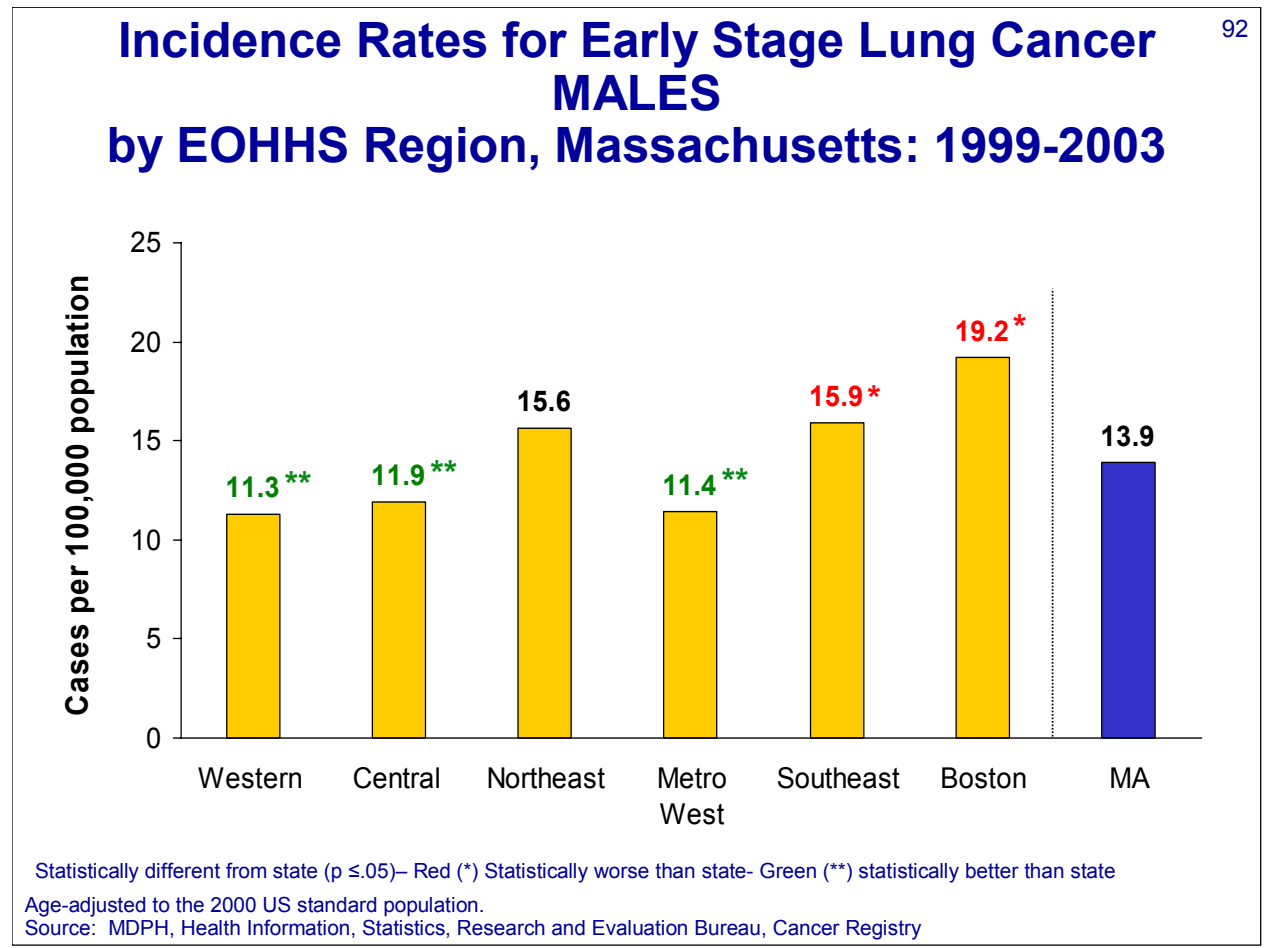



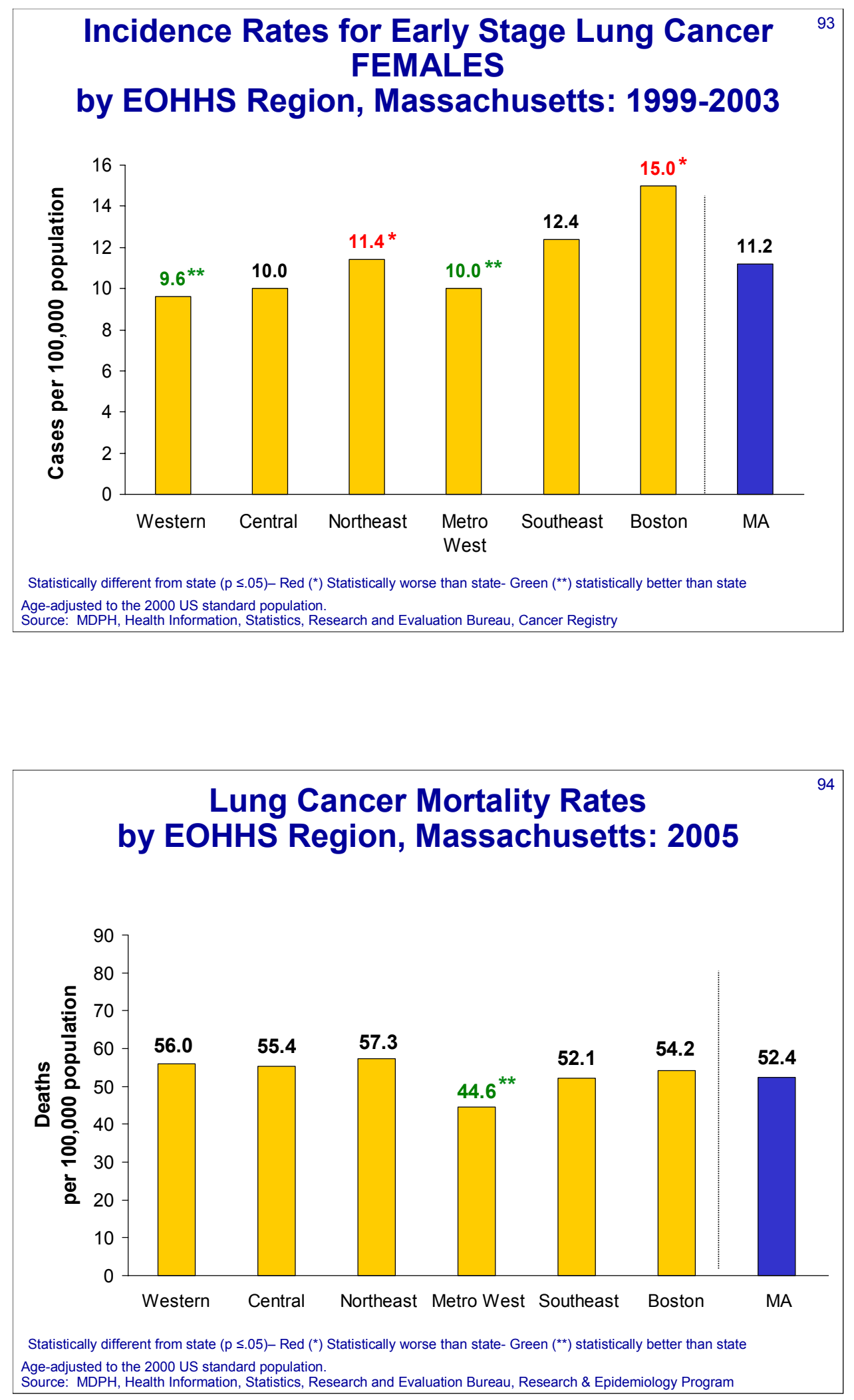
Lung Cancer Mortality Rate by

Cities in Boston Region \& Massachusetts: 2003-2005

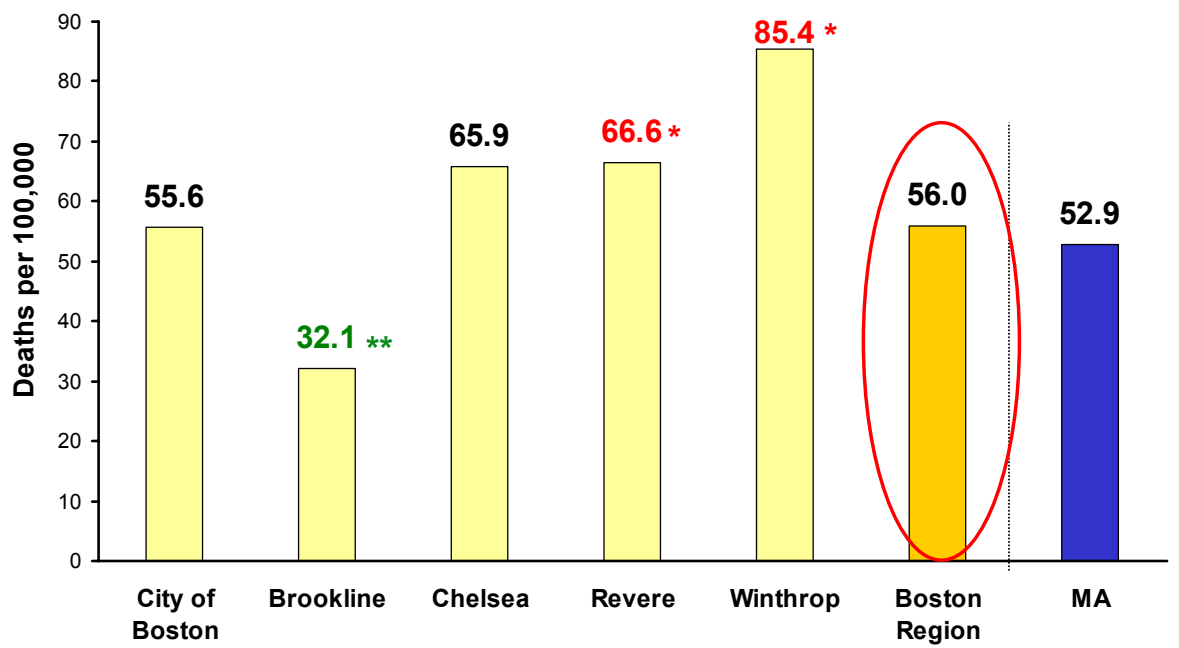

Statistically different from state $(p \leq .05)-$ Red $\left(^{*}\right)$ Statistically worse than state- Green $\left(^{* \star}\right)$ statistically better than state

Age-adjusted to the 2000 US standard population

Source: MDPH, Health Information, Statistics, Research and Evaluation Bureau, Research \& Epidemiology Program

Lung Cancer Mortality Rates by Race and Ethnicity, Boston Region \& Massachusetts: 2003-2005

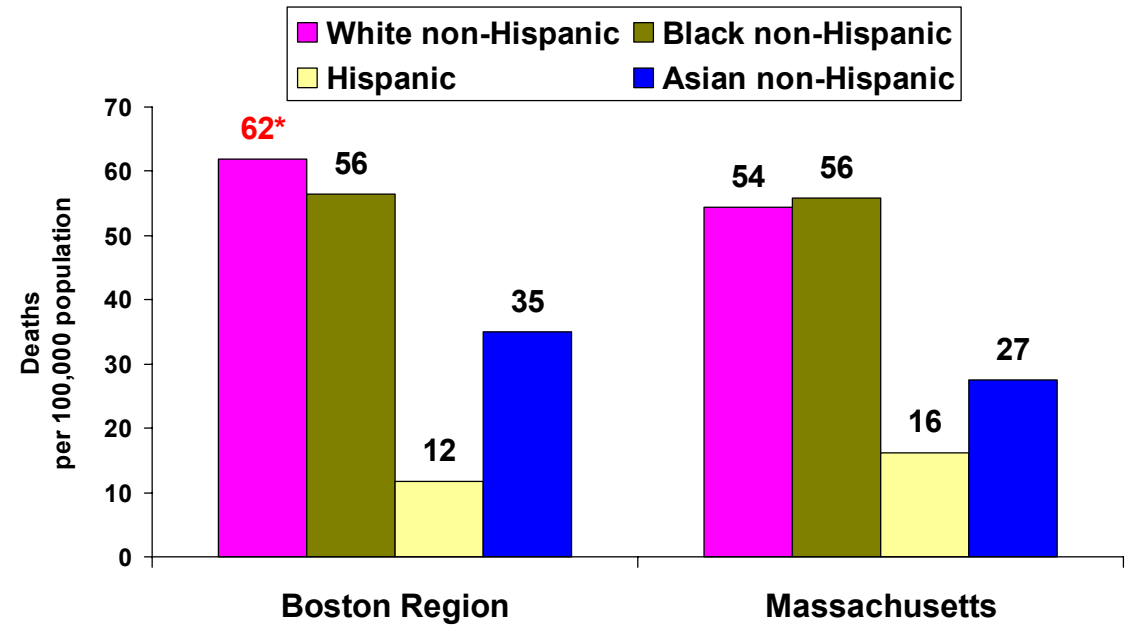

Statistically different from state $(p \leq .05)-$ Red $\left({ }^{*}\right)$ Statistically worse than state- Green $\left(^{\star \star}\right)$ statistically better than state Age-adjusted to the 2000 US standard population.

Source: Division of Health Care Finance and Policy. Calendar Year 2003- 2005. Hospital Discharges 


\section{Adults who Currently Smoke by EOHHS Region, Massachusetts: 2005}

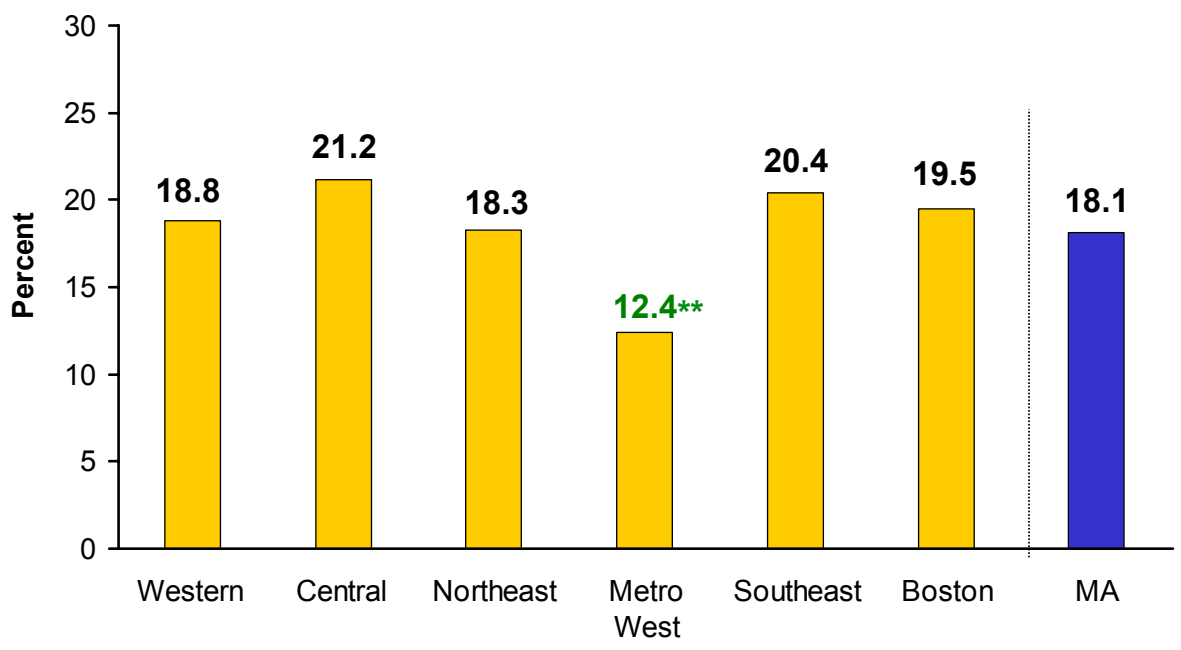

Statistically different from state $(p \leq .05)-\operatorname{Red}\left({ }^{*}\right)$ Statistically worse than state- Green $\left({ }^{* *}\right)$ statistically better than state

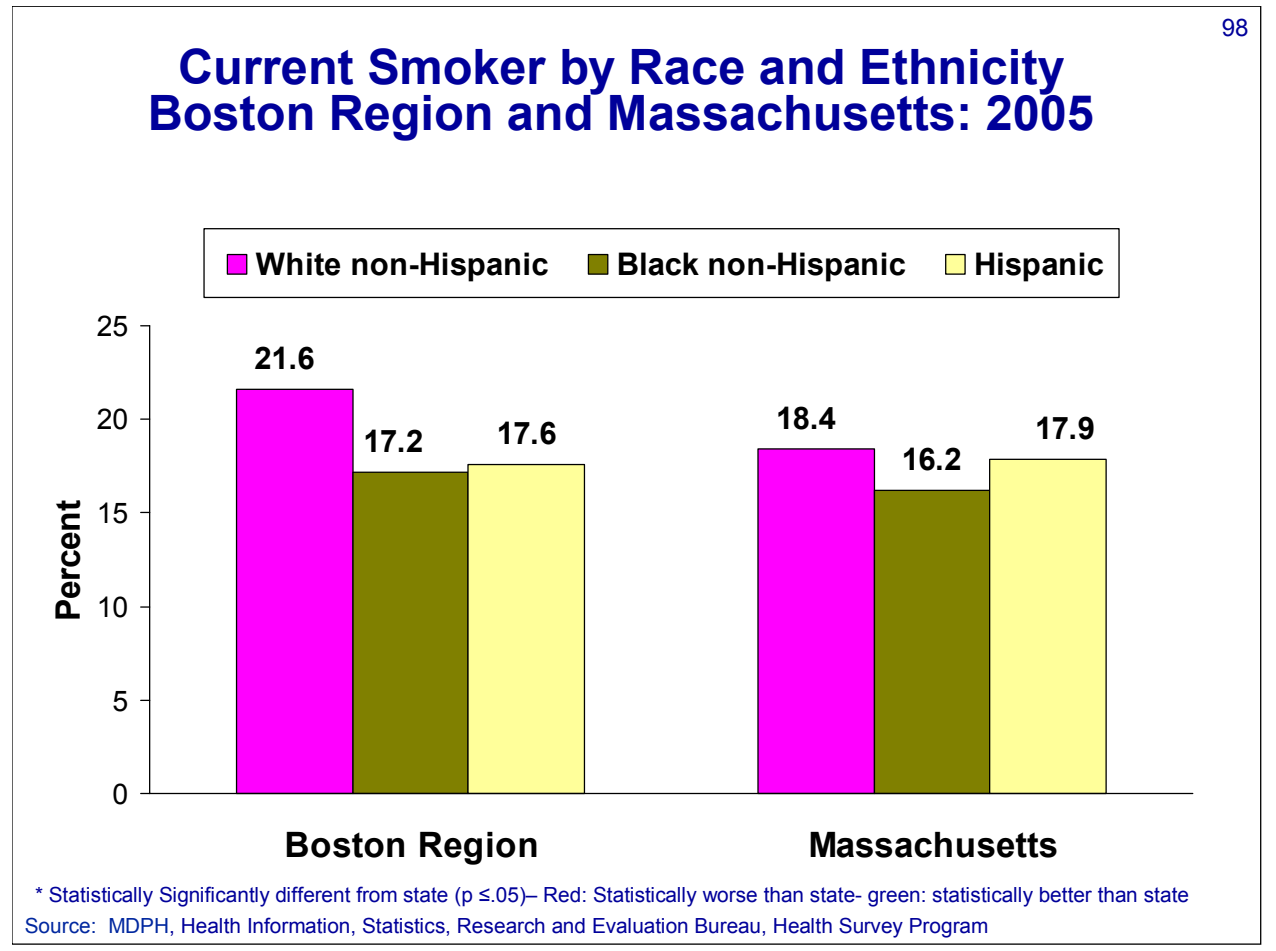


Summary Indicators for Cancer Screening by EOHHS Region, Massachusetts: 2005

\begin{tabular}{|c|c|c|c|c|c|c|c|}
\hline Indicator & $\begin{array}{c}\text { MA } \\
\text { Total }\end{array}$ & Western & Central & $\begin{array}{c}\text { North } \\
\text { East }\end{array}$ & $\begin{array}{c}\text { Metro } \\
\text { West }\end{array}$ & $\begin{array}{c}\text { South } \\
\text { East }\end{array}$ & Boston \\
\hline $\begin{array}{c}\text { \% Men }{ }^{\dagger} \\
\text { PSA (2004) }\end{array}$ & $\mathbf{5 6 . 1}$ & 49.1 & 52.3 & 52.3 & 62.5 & 56.3 & 66.2 \\
\hline $\begin{array}{c}\text { \% Men }{ }^{\dagger} \\
\text { DRE (2004) }\end{array}$ & $\mathbf{6 2 . 7}$ & 59.5 & 60.0 & 63.0 & 64.5 & 62.6 & 69.0 \\
\hline $\begin{array}{c}\text { \% Blood Stool Test } \\
\text { † }\end{array}$ & $\mathbf{3 0 . 0}$ & 34.2 & 29.9 & 33.5 & 30.9 & 26.4 & 20.7 \\
\hline $\begin{array}{c}\text { \% Adults } \\
\text { Sigmoidoscopy or } \\
\text { Colonoscopy' }\end{array}$ & $\mathbf{5 8 . 8}$ & 53.9 & 60.2 & 53.2 & 68.5 & 55 & 58.9 \\
\hline $\begin{array}{c}\text { \% Women Clinical } \\
\text { Breast Exam (2004) }\end{array}$ & $\mathbf{8 6 . 6}$ & 85.0 & 85.5 & 90.0 & 87.1 & 85.6 & 85.7 \\
\hline $\begin{array}{c}\text { \% Women* } \\
\text { Mammography }\end{array}$ & $\mathbf{8 4 . 1}$ & 86.9 & 83.0 & 83.4 & 87.1 & 82.0 & 80.1 \\
\hline
\end{tabular}

*40 years and older, $\uparrow 50$ years and older

Better Outcome (significantly different from state)

\begin{tabular}{|c|c|c|c|c|c|c|c|}
\hline \multicolumn{8}{|c|}{$\begin{array}{l}\text { Summary Cancer Indicators (Early Stage) } \\
\text { by EOHHS Region, Massachusetts: 1999-2003 }\end{array}$} \\
\hline Indicator & $\begin{array}{c}\text { MA } \\
\text { Total }\end{array}$ & Western & Central & Northeast & $\begin{array}{l}\text { Metro } \\
\text { West }\end{array}$ & $\begin{array}{c}\text { South } \\
\text { East }\end{array}$ & Boston \\
\hline $\begin{array}{l}\text { Prostate } \\
\text { Cancer } \\
\text { Incidence }\end{array}$ & 140.5 & 129.7 & 122.4 & 140.1 & 128.5 & 156.6 & 174.6 \\
\hline $\begin{array}{c}\text { Female } \\
\text { Breast } \\
\text { Cancer } \\
\text { Incidence }\end{array}$ & 118.6 & 112.9 & 105.3 & 124.9 & 119.8 & 113.1 & 137.6 \\
\hline $\begin{array}{l}\text { Colorectal } \\
\text { Cancer } \\
\text { Incidence }\end{array}$ & 26.9 & 25.9 & 21.3 & 29.5 & 24.3 & 28.7 & 32.0 \\
\hline $\begin{array}{l}\text { Lung Cancer } \\
\text { Incidence }\end{array}$ & 14.4 & 12.0 & 12.7 & 15.3 & 12.4 & 16.1 & 19.6 \\
\hline
\end{tabular}


Summary Cancer Indicators (Late Stage) by EOHHS Region, Massachusetts: 1999-2003

\begin{tabular}{|c|c|c|c|c|c|c|c|}
\hline Indicator & Motal & Western & Central & Northeast & $\begin{array}{c}\text { Metro } \\
\text { West }\end{array}$ & $\begin{array}{c}\text { South } \\
\text { East }\end{array}$ & Boston \\
\hline $\begin{array}{c}\text { Prostate } \\
\text { Cancer } \\
\text { Incidence }\end{array}$ & $\mathbf{5 . 5}$ & 4.9 & 5.2 & 5.9 & 3.9 & 6.2 & 8.7 \\
\hline $\begin{array}{c}\text { Female } \\
\text { Breast } \\
\text { Cancer } \\
\text { Incidence }\end{array}$ & $\mathbf{3 7 . 8}$ & 37.9 & 31.7 & 37.0 & 36.8 & 38.4 & 47.3 \\
\hline $\begin{array}{c}\text { Colorectal } \\
\text { Cancer } \\
\text { Incidence }\end{array}$ & $\mathbf{3 3 . 8}$ & 30.6 & 32.9 & 35.2 & 30.6 & 34.4 & 42.7 \\
\hline $\begin{array}{c}\text { Lung Cancer } \\
\text { Incidence }\end{array}$ & $\mathbf{5 0 . 3}$ & 45.8 & 48.6 & 54.1 & 41.4 & 55.1 & 63.2 \\
\hline
\end{tabular}

\begin{tabular}{|c|c|c|c|c|c|c|c|}
\hline \multicolumn{8}{|c|}{$\begin{array}{l}\text { Summary Invasive Cancer Incidence Rates } \\
\text { by EOHHS Region, Massachusetts: 1999-2003 }\end{array}$} \\
\hline Indicator & $\begin{array}{c}\text { MA } \\
\text { Total }\end{array}$ & Western & Central & Northeast & $\begin{array}{l}\text { Metro } \\
\text { West }\end{array}$ & $\begin{array}{c}\text { South } \\
\text { East }\end{array}$ & Boston \\
\hline $\begin{array}{l}\text { Prostate } \\
\text { Cancer } \\
\text { Incidence }\end{array}$ & 181.9 & 170.3 & 159.7 & 180.5 & 178.4 & 202.5 & 191.3 \\
\hline $\begin{array}{c}\text { Female } \\
\text { Breast } \\
\text { Cancer } \\
\text { Incidence }\end{array}$ & 138.9 & 135.3 & 127.8 & 139.8 & 146.7 & 139.6 & 134.9 \\
\hline $\begin{array}{l}\text { Colorectal } \\
\text { Cancer } \\
\text { Incidence }\end{array}$ & 58.9 & 56.2 & 55.2 & 61.8 & 57.2 & 59.7 & 63.6 \\
\hline $\begin{array}{l}\text { Lung Cancer } \\
\text { Incidence }\end{array}$ & 72.0 & 67.2 & 69.9 & 76.7 & 65.1 & 77.5 & 77.7 \\
\hline
\end{tabular}


Summary Cancer Mortality Indicators by EOHHS Region, Massachusetts: 2005

\begin{tabular}{||c|c|c|c|c|c|c|c|}
\hline Indicator & $\begin{array}{c}\text { MA } \\
\text { Total }\end{array}$ & Western & Central & Northeast & $\begin{array}{c}\text { Metro } \\
\text { West }\end{array}$ & $\begin{array}{c}\text { South } \\
\text { East }\end{array}$ & Boston \\
\hline $\begin{array}{c}\text { Prostate } \\
\text { Cancer Death } \\
\text { Rate }\end{array}$ & $\mathbf{2 1 . 8}$ & 25.0 & 27.7 & 22.9 & 18.7 & 17.9 & 24.0 \\
\hline $\begin{array}{c}\text { Colorectal } \\
\text { Cancer Death } \\
\text { Rate }\end{array}$ & 17.4 & 18.2 & 18.3 & 17.5 & 16.4 & 17.2 & 18.4 \\
\hline $\begin{array}{c}\text { Female } \\
\text { Breast } \\
\text { Cancer Death } \\
\text { Rate }\end{array}$ & $\mathbf{2 3 . 0}$ & 25.5 & 23.1 & 23.1 & 20.9 & 22.5 & 24.6 \\
\hline $\begin{array}{c}\text { Lung Cancer } \\
\text { Death Rate }\end{array}$ & $\mathbf{5 2 . 4}$ & 56.0 & 55.4 & 57.3 & 44.6 & 52.1 & 54.2 \\
\hline
\end{tabular}


SUBSTANCE ABUSE 
Alcohol Use in Past Month Persons Aged 12 to 20 by EOHHS Region, Massachusetts: 2002, 2003, 2004

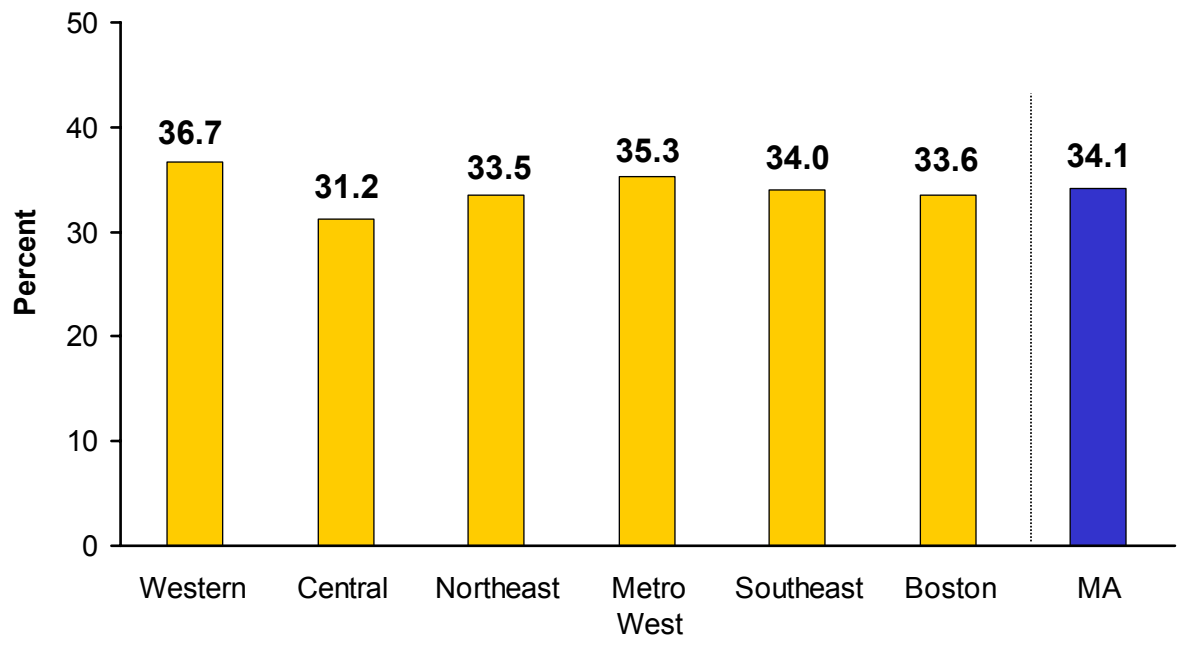

Statistically different from state $(p \leq .05)-\operatorname{Red}\left({ }^{*}\right)$ Statistically worse than state- Green $\left(^{* *}\right)$ statistically better than state Source: SAMHSA, Office of Applied Studies, National Survey on Drug Use and Health, 2002, 2003, and 2004.

\section{Binge Drinking ${ }^{1}$ in Past Month Persons Aged 12 to 20 by EOHHS Region, Massachusetts: 2002, 2003, 2004}

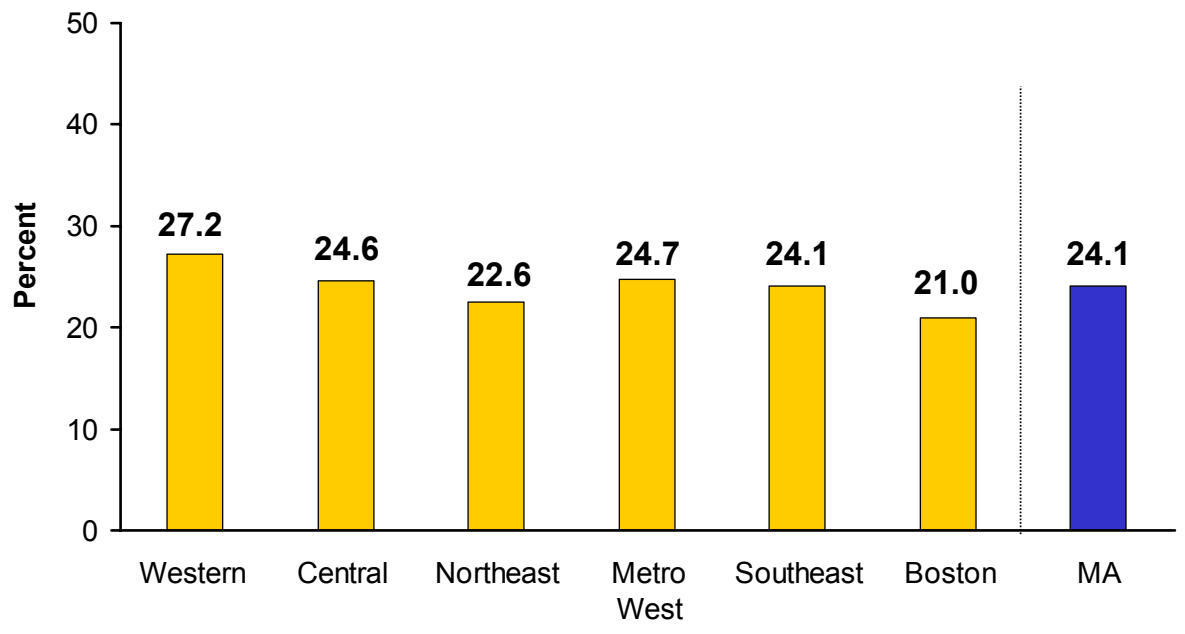

Statistically different from state $(p \leq .05)-\operatorname{Red}\left({ }^{*}\right)$ Statistically worse than state- Green $\left(^{* *}\right)$ statistically better than state

${ }^{1}$ Binge Alcohol use is defined as drinking $5+$ drinks on the same occasion on at least 1 day in the past 30 days

Source: SAMHSA, Office of Applied Studies, National Survey on Drug Use and Health, 2002, 2003, and 2004. 
Any Illicit Drug Use in Past Month Persons Aged 12+ by EOHHS Region, Massachusetts: 2002, 2003, 2004

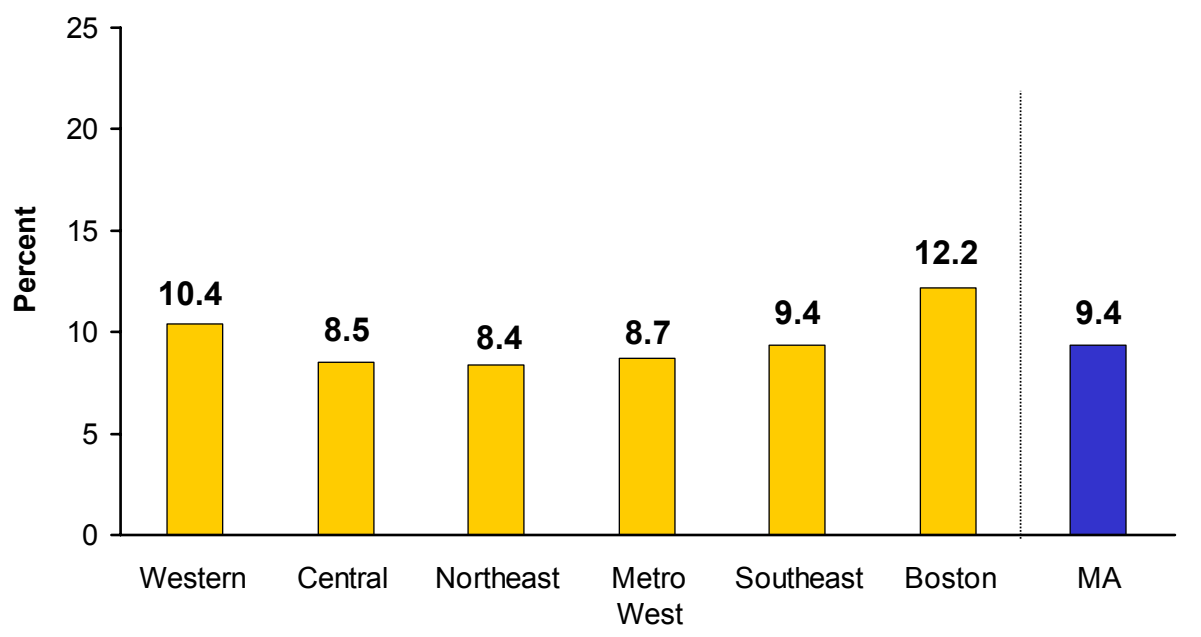

Statistically different from state $(p \leq .05)-\operatorname{Red}\left({ }^{*}\right)$ Statistically worse than state- Green $\left(^{* *}\right)$ statistically better than state Source: SAMHSA, Office of Applied Studies, National Survey on Drug Use and Health, 2002, 2003, and 2004.

Any Illicit Drug Use Other than Marijuana in Past Month Persons Aged 12+ by EOHHS Region Massachusetts: 2002, 2003, 2004

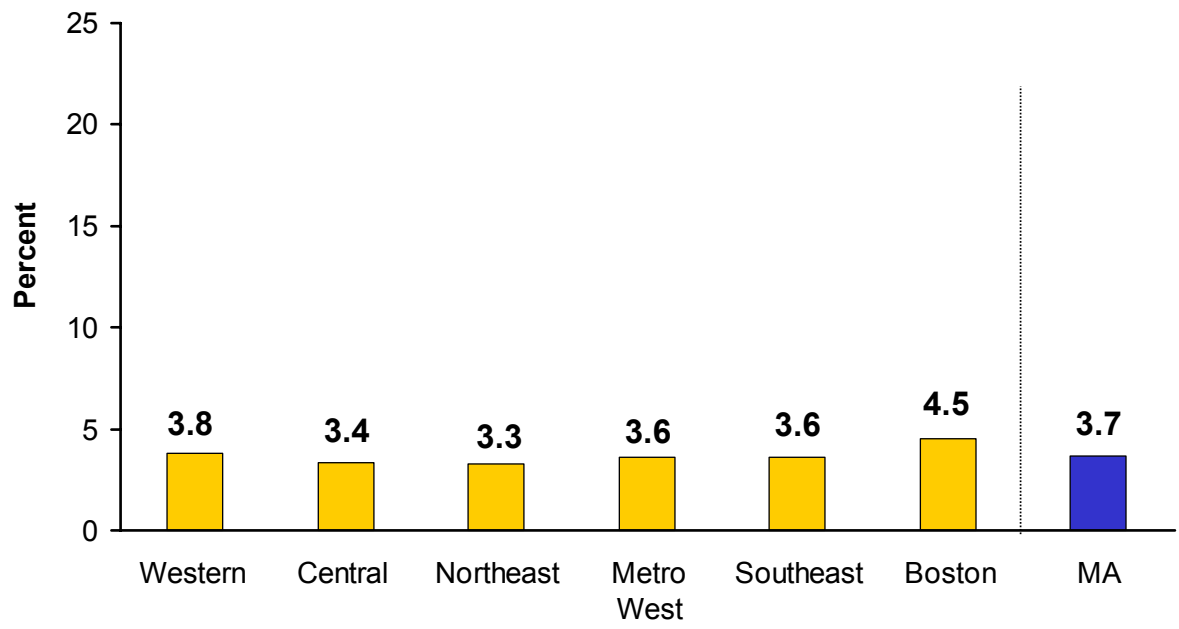

Statistically different from state $(p \leq .05)-$ Red $\left({ }^{*}\right)$ Statistically worse than state- Green $\left({ }^{\star *}\right)$ statistically better than state Source: SAMHSA, Office of Applied Studies, National Survey on Drug Use and Health, 2002, 2003, and 2004. 
Alcohol Dependence or Abuse in Past Year Persons Aged 12+ by EOHHS Region

Massachusetts: 2002, 2003, 2004

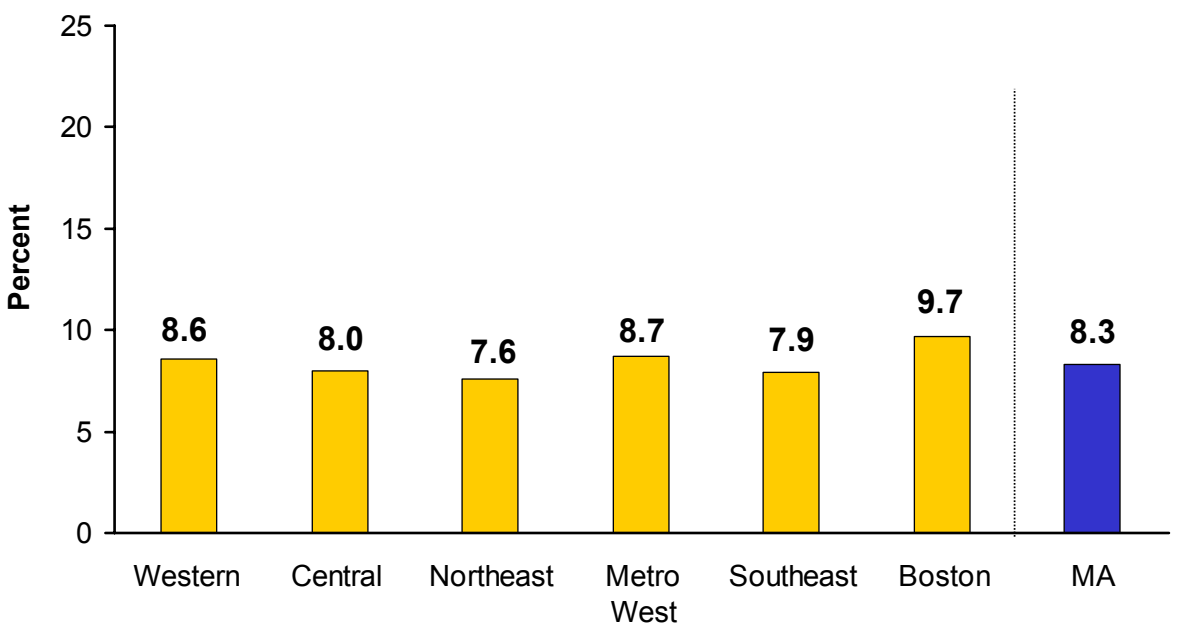

Statistically different from state $(p \leq .05)-\operatorname{Red}\left({ }^{*}\right)$ Statistically worse than state- Green $\left(^{* *}\right)$ statistically better than state Source: SAMHSA, Office of Applied Studies, National Survey on Drug Use and Health, 2002, 2003, and 2004.

Dependence or Abuse of Any Illicit Drug or Alcohol in

Past Year Persons Aged 12+ by EOHHS Region

Massachusetts: 2002, 2003, 2004

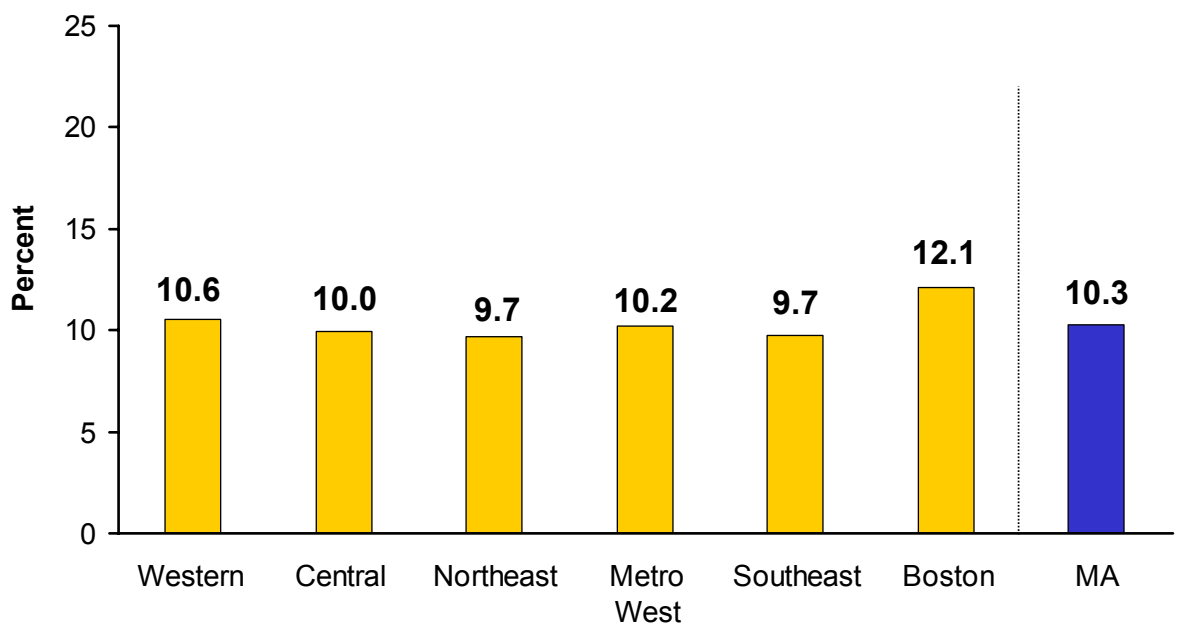

Statistically different from state $(p \leq .05)-$ Red $\left({ }^{*}\right)$ Statistically worse than state- Green $\left(^{* *}\right)$ statistically better than state Source: SAMHSA, Office of Applied Studies, National Survey on Drug Use and Health, 2002, 2003, and 2004. 


\section{Adults who Binge Drink \\ by EOHHS Region, Massachusetts: 2005}

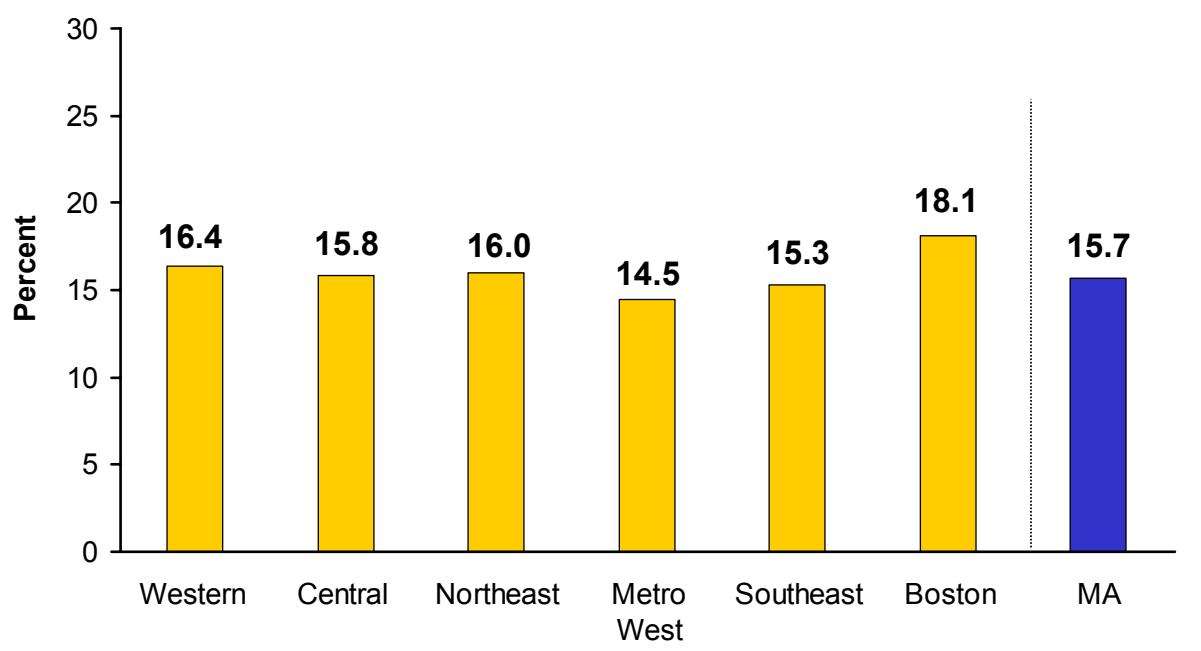

Statistically different from state $(p \leq .05)-\operatorname{Red}\left({ }^{*}\right)$ Statistically worse than state- Green $\left(^{* *}\right)$ statistically better than state Source: MDPH, Health Information, Statistics, Research and Evaluation Bureau, Health Survey Program

\section{Adults who Used Illicit Drugs in Past Month by EOHHS Region, Massachusetts: 2005}

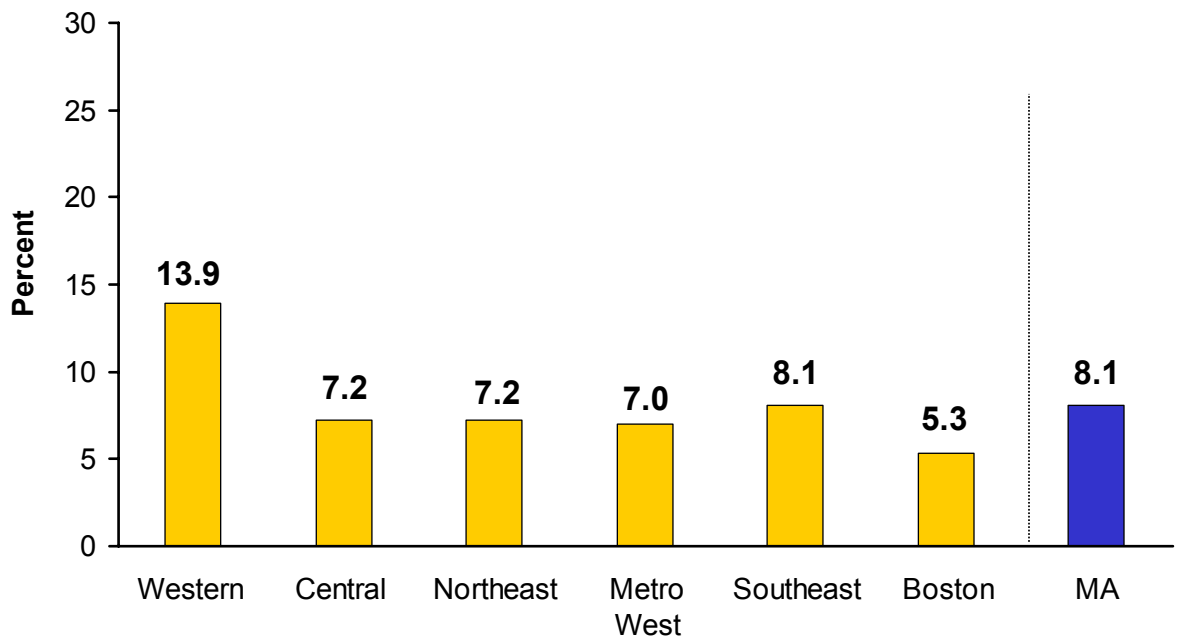

Statistically different from state $(p \leq .05)-\operatorname{Red}\left({ }^{*}\right)$ Statistically worse than state- Green $\left(^{* *}\right)$ statistically better than state 

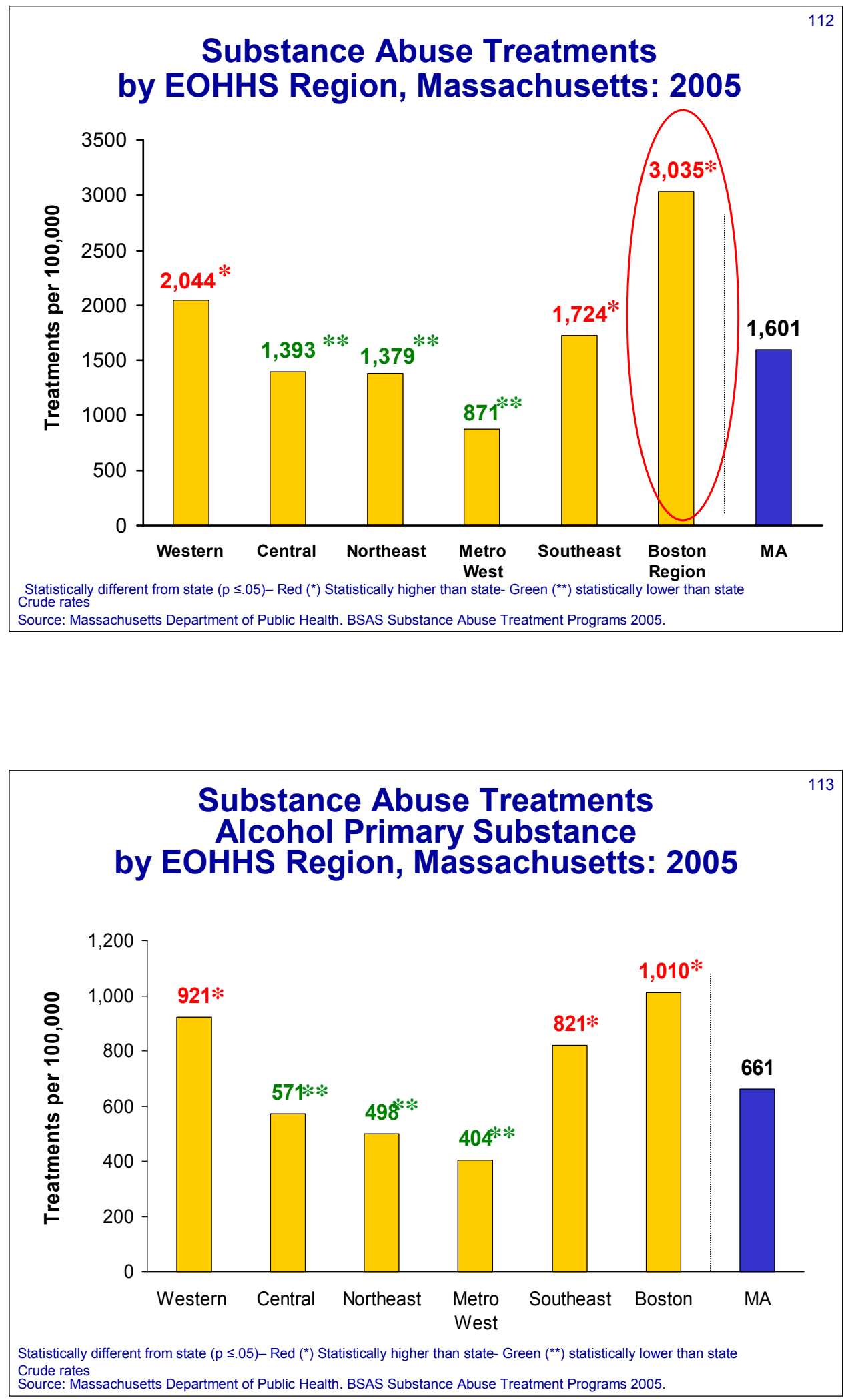


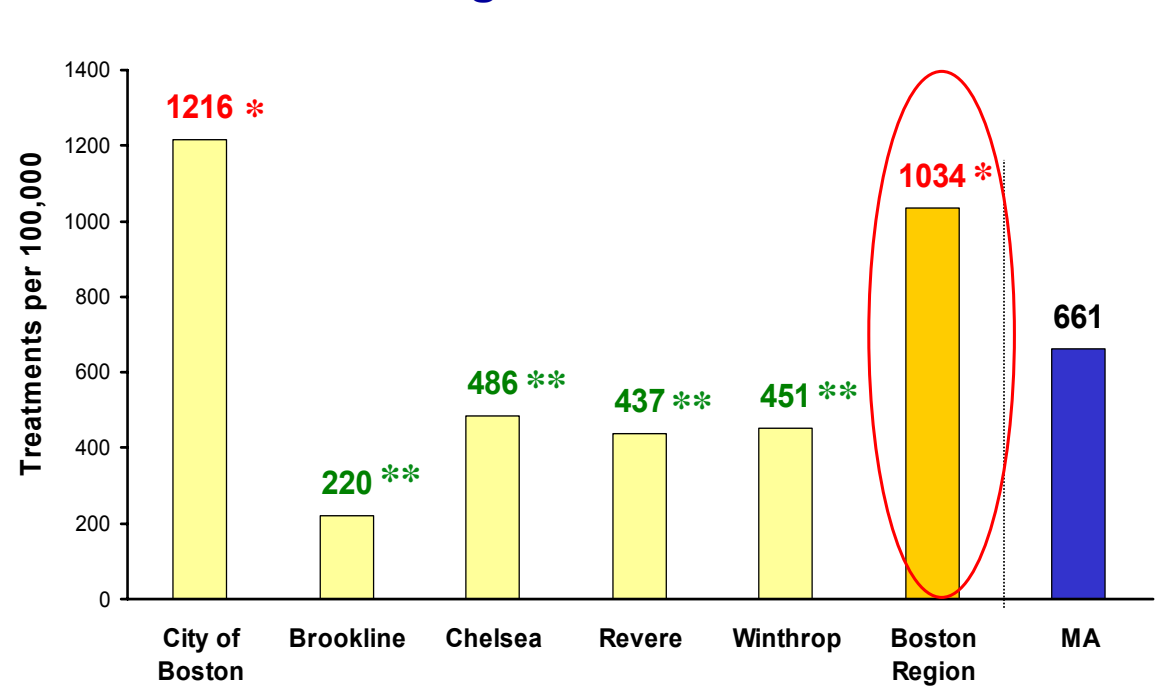

Statistically different from state $(p \leq .05)-\operatorname{Red}\left({ }^{*}\right)$ Statistically worse than state- Green $\left(^{\star *}\right)$ statistically better than state

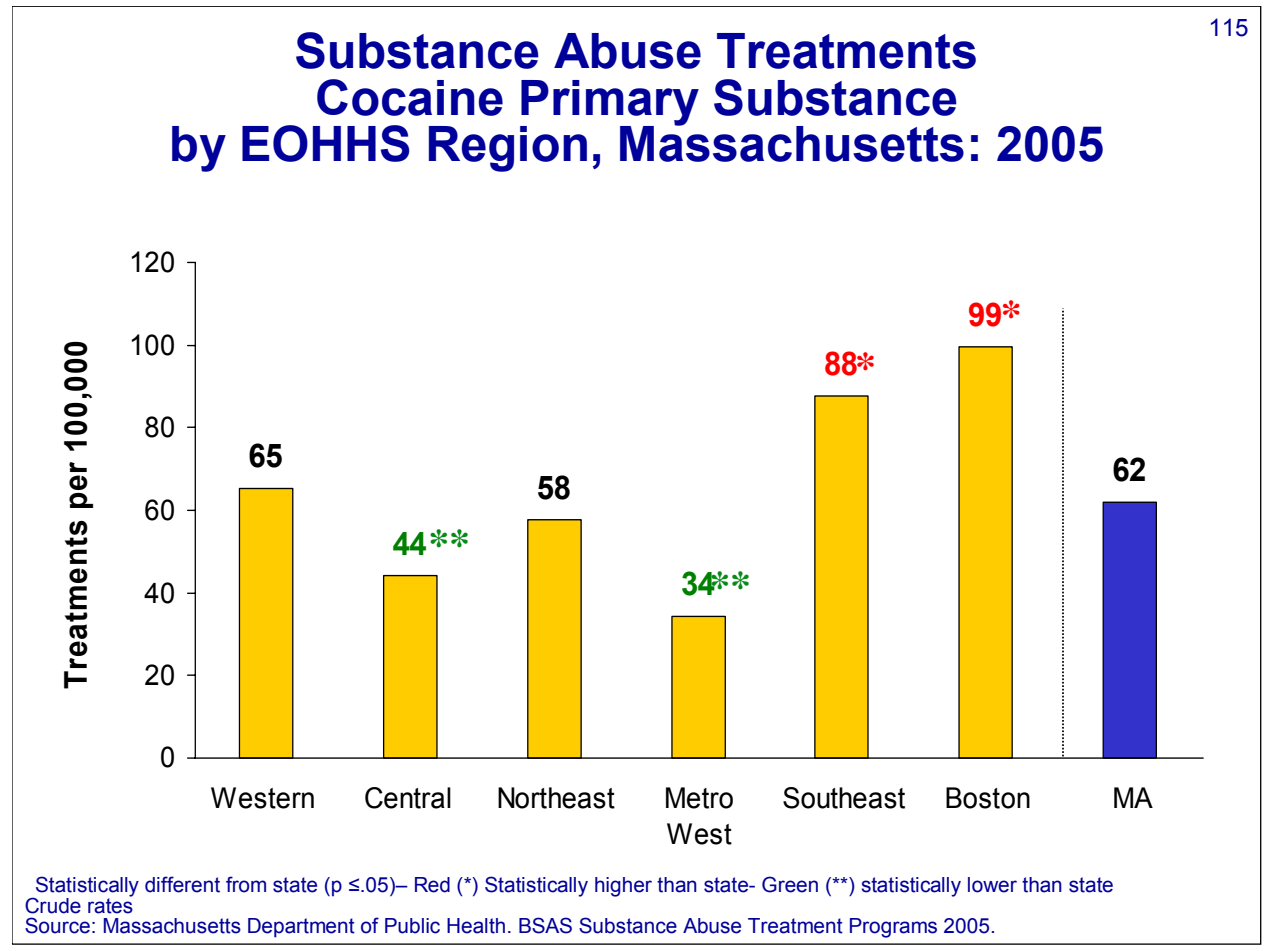



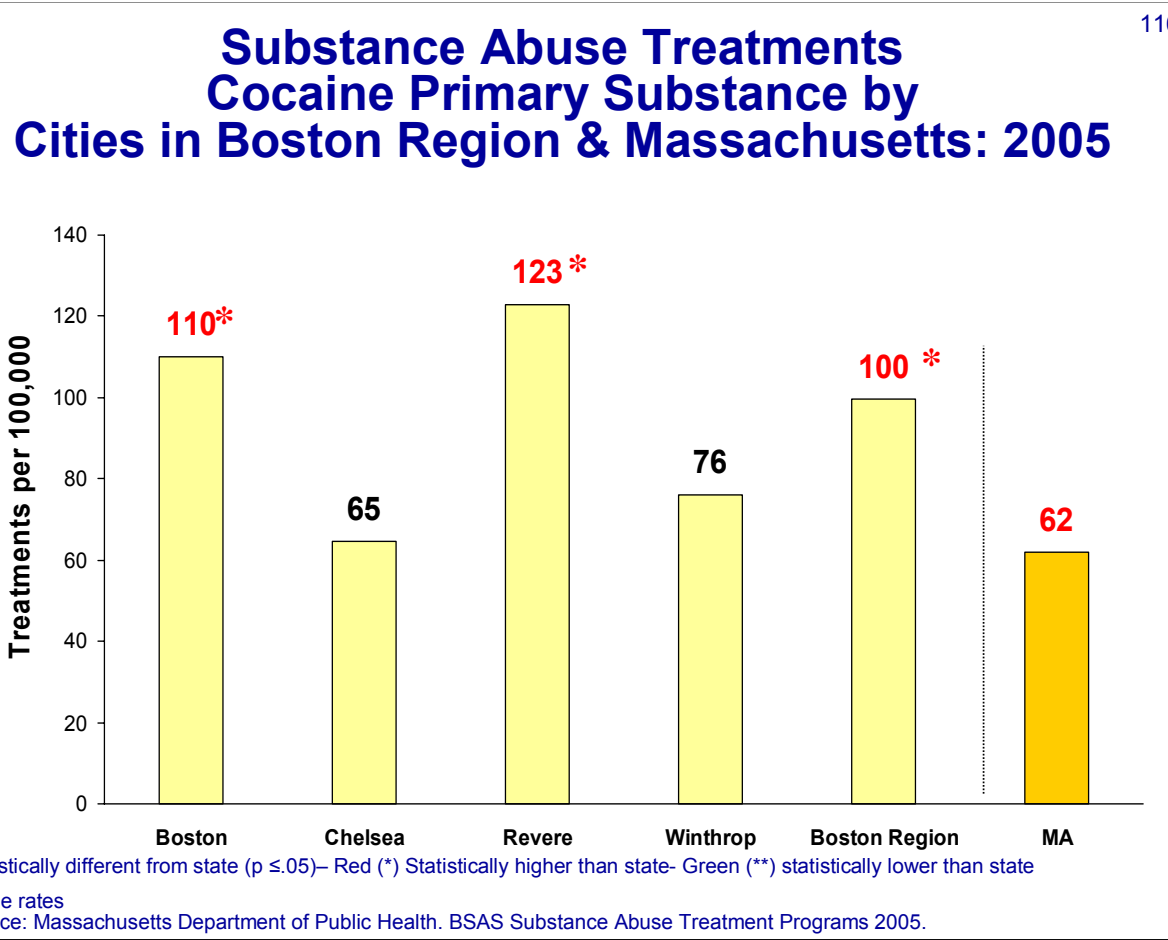

Crude rates

Source: Massachusetts Department of Public Health. BSAS Substance Abuse Treatment Programs 2005

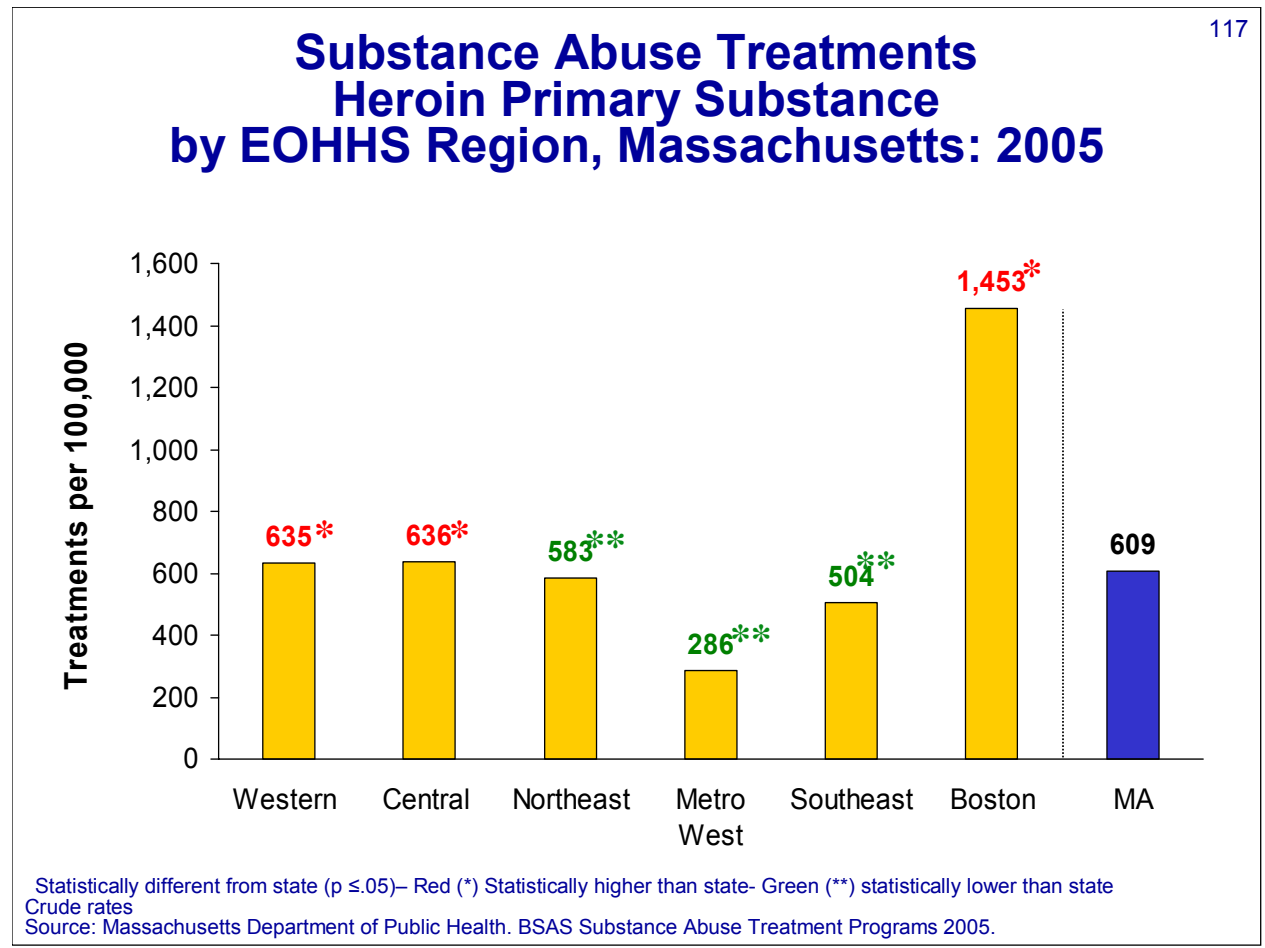




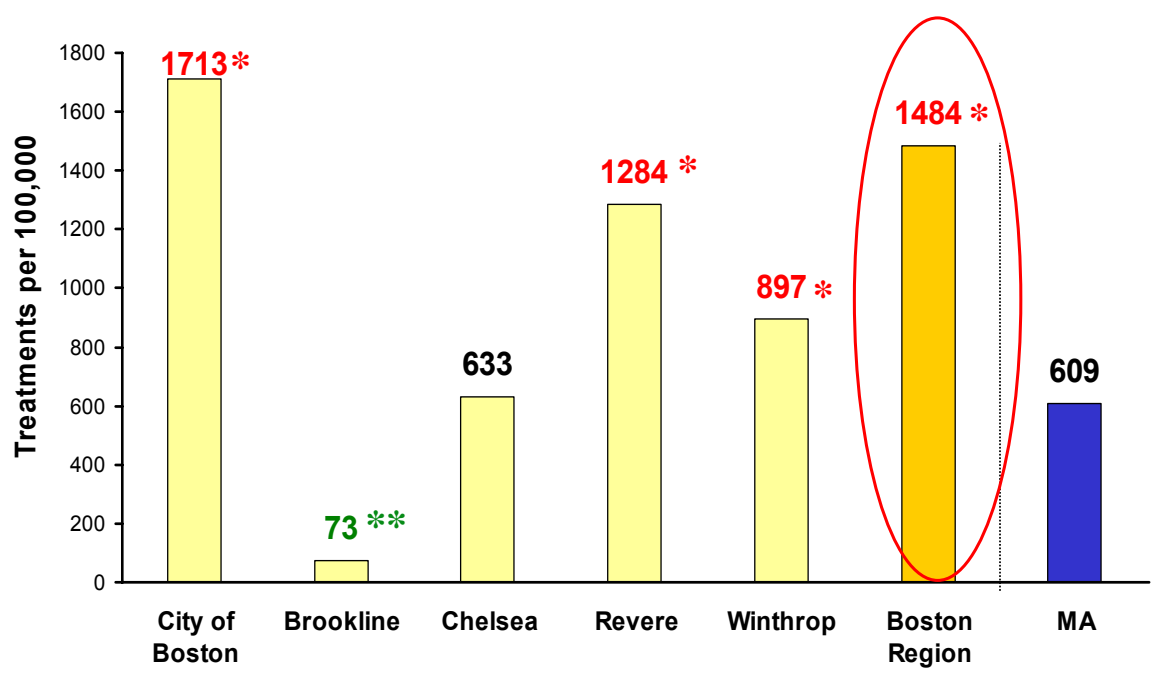

Statistically different from state $(p \leq .05)-\operatorname{Red}\left({ }^{*}\right)$ Statistically worse than state- Green $\left(^{\star \star}\right)$ statistically better than state

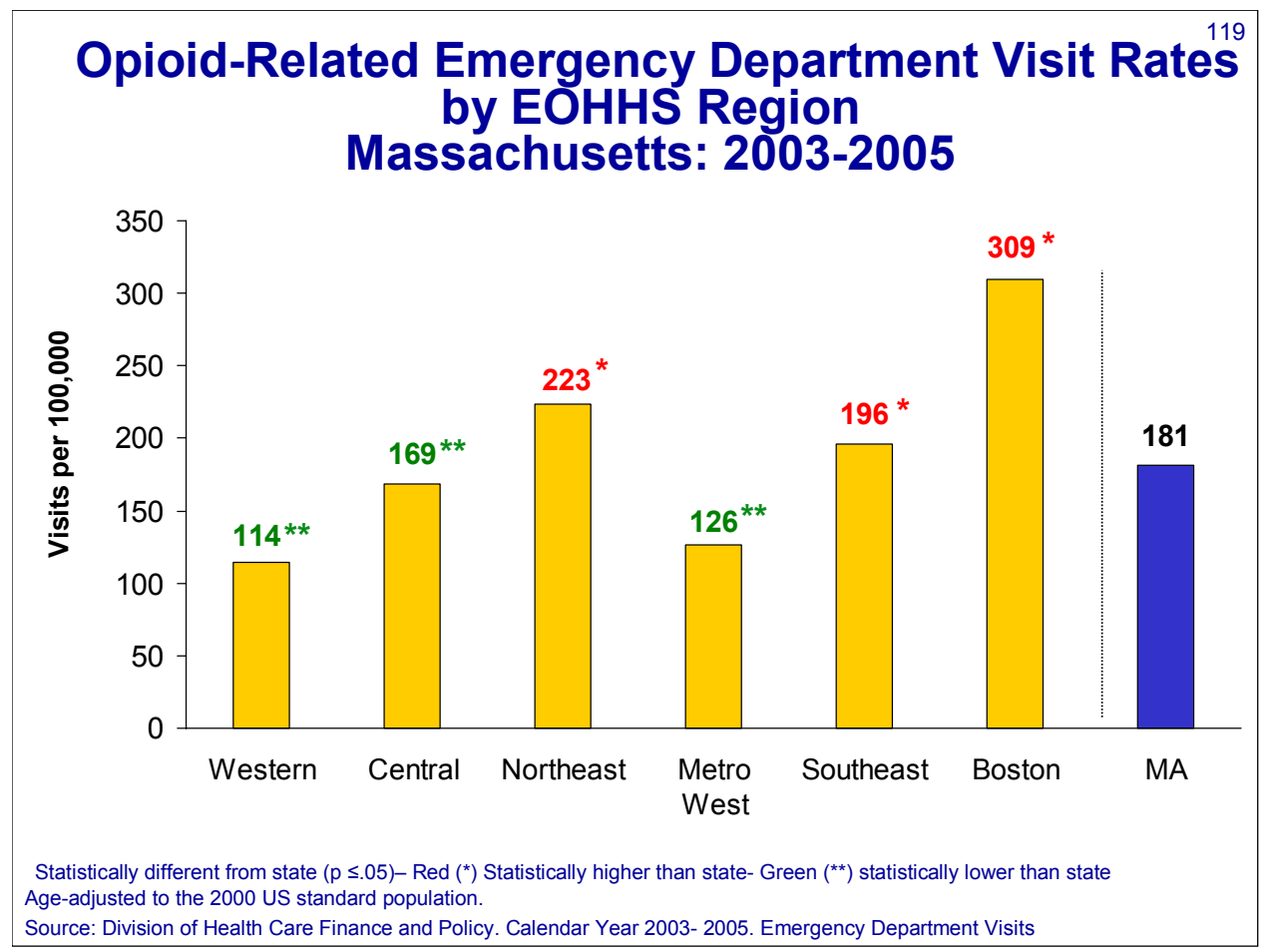




\section{Opioid-Related Emergency Department Visit Rates \\ by Boston Cities and Massachusetts: 2005}

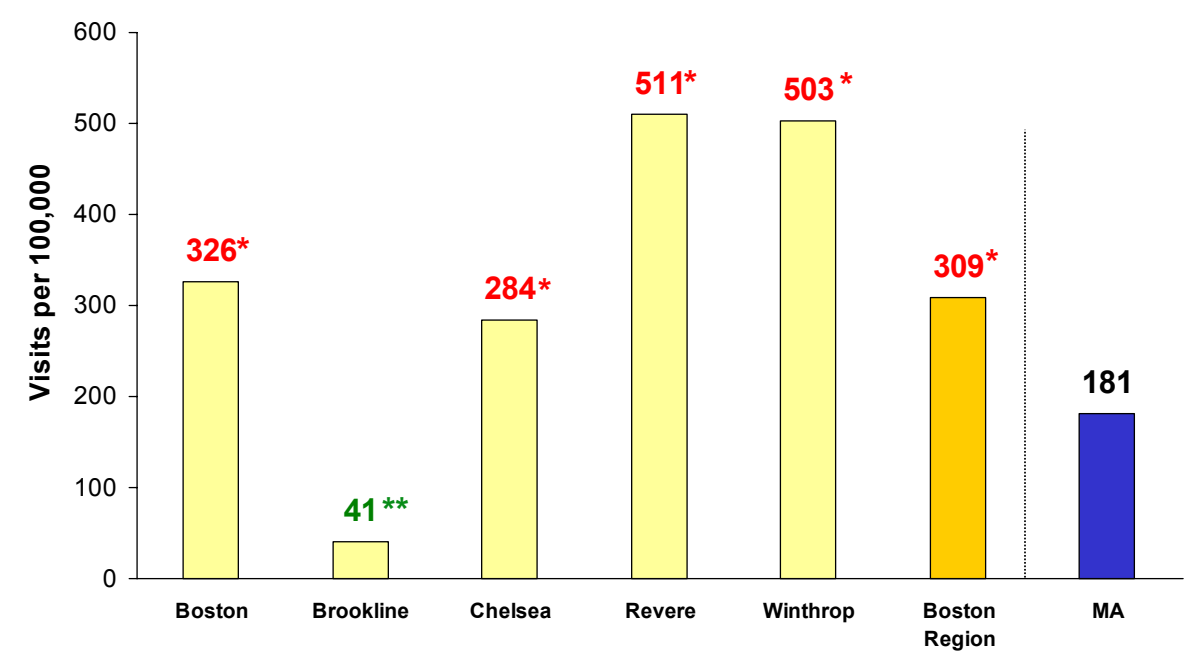

Statistically different from state $(p \leq .05)-\operatorname{Red}\left({ }^{*}\right)$ Statistically worse than state- Green $\left({ }^{* *}\right)$ statistically better than state

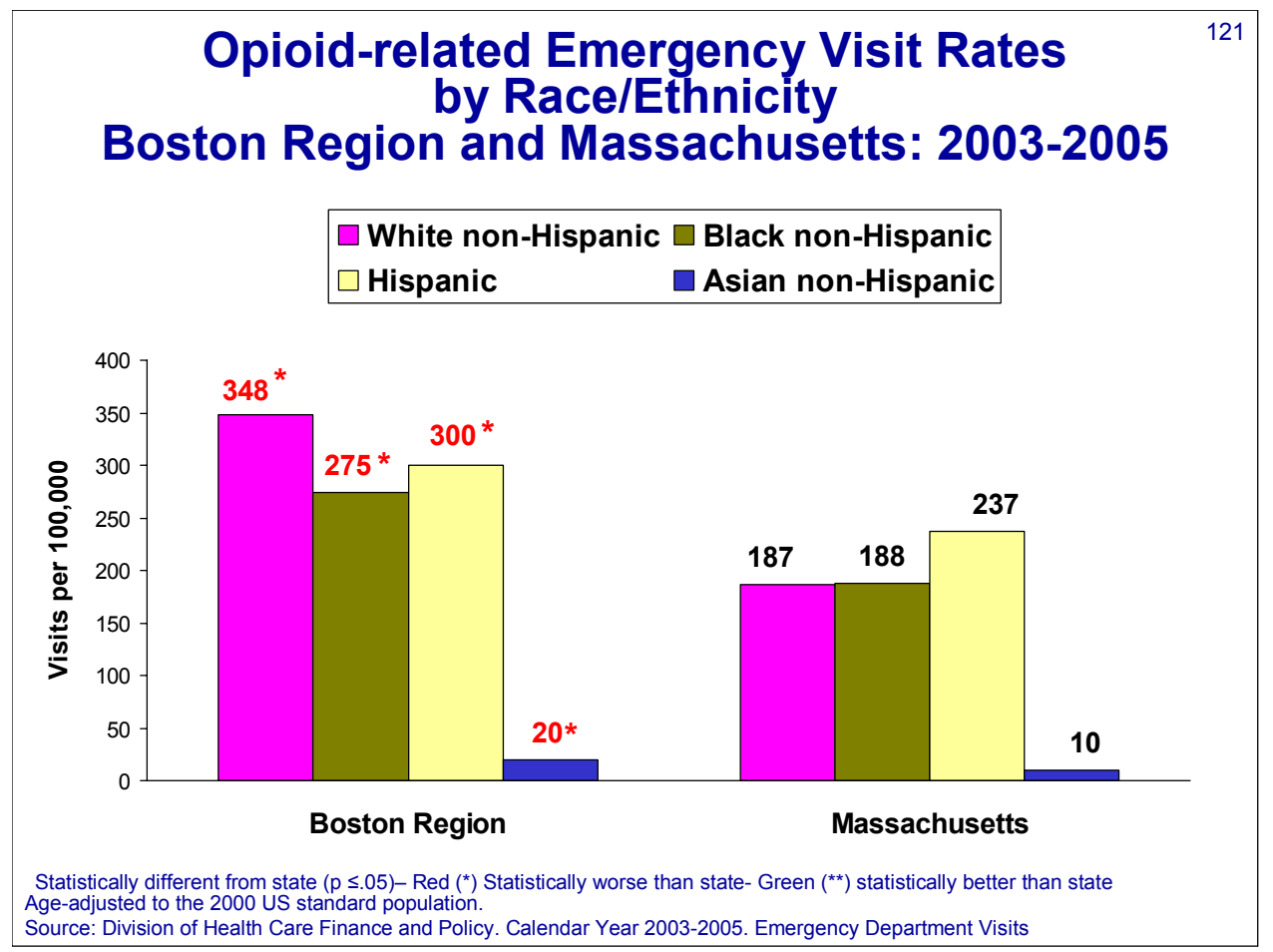



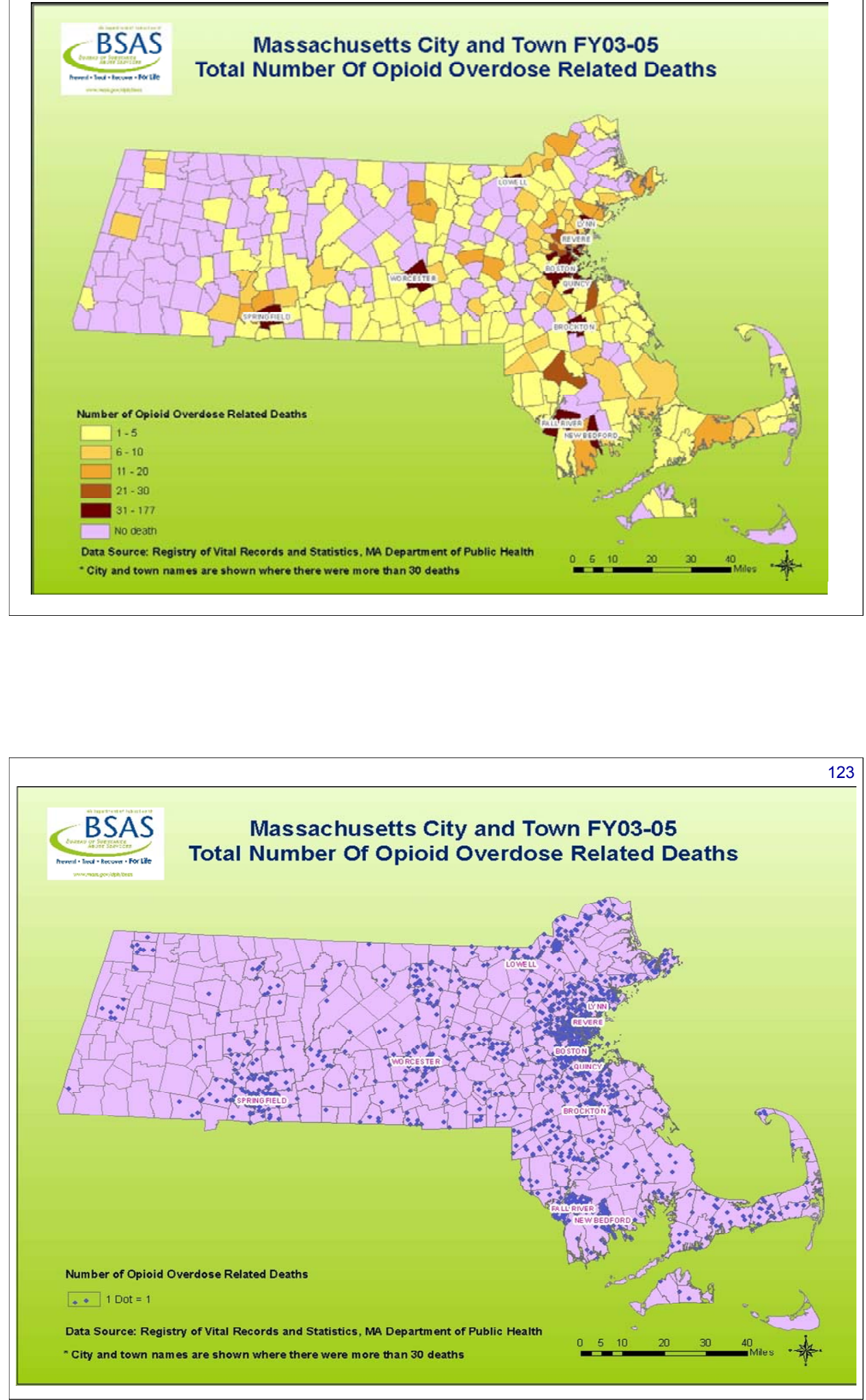

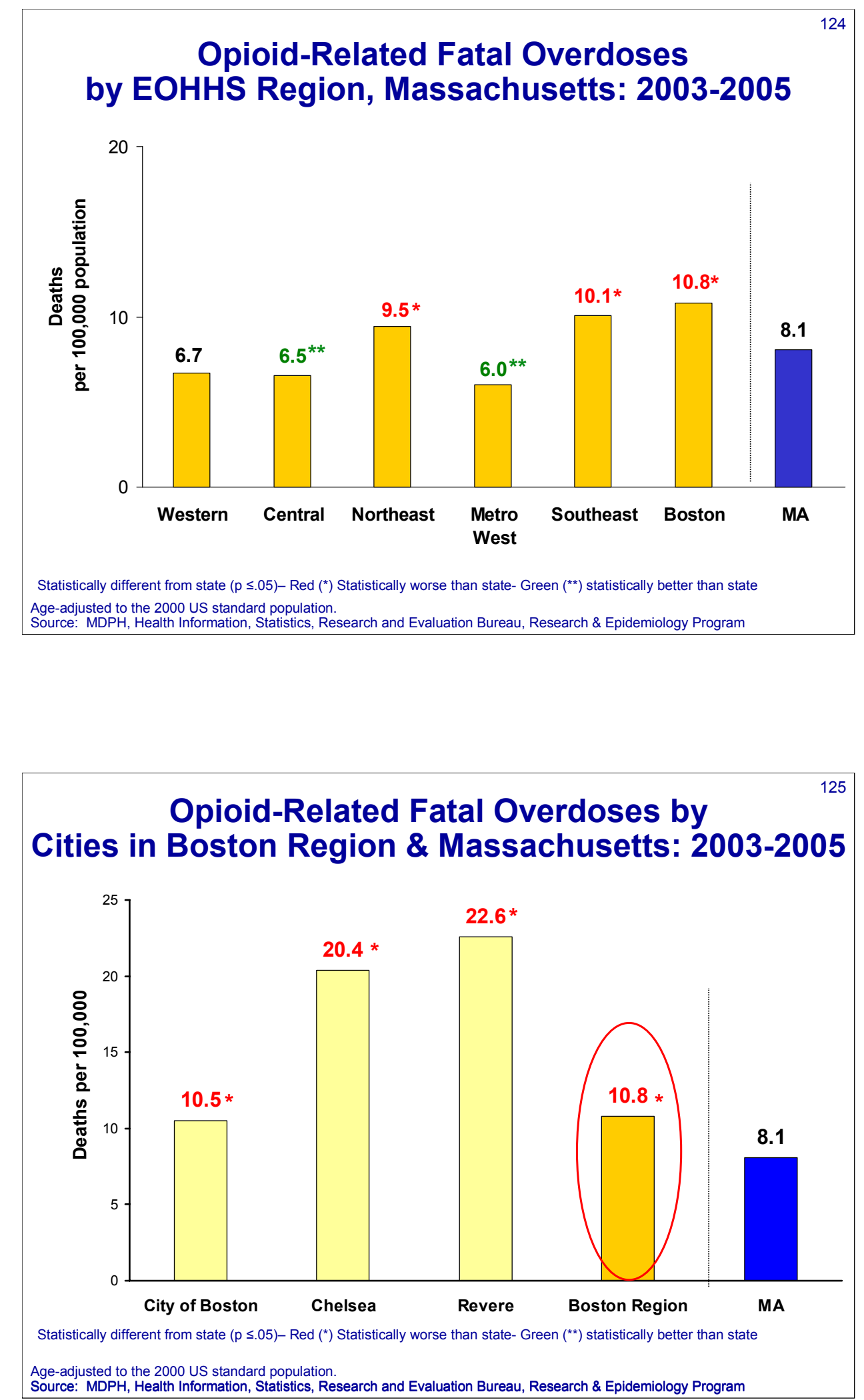

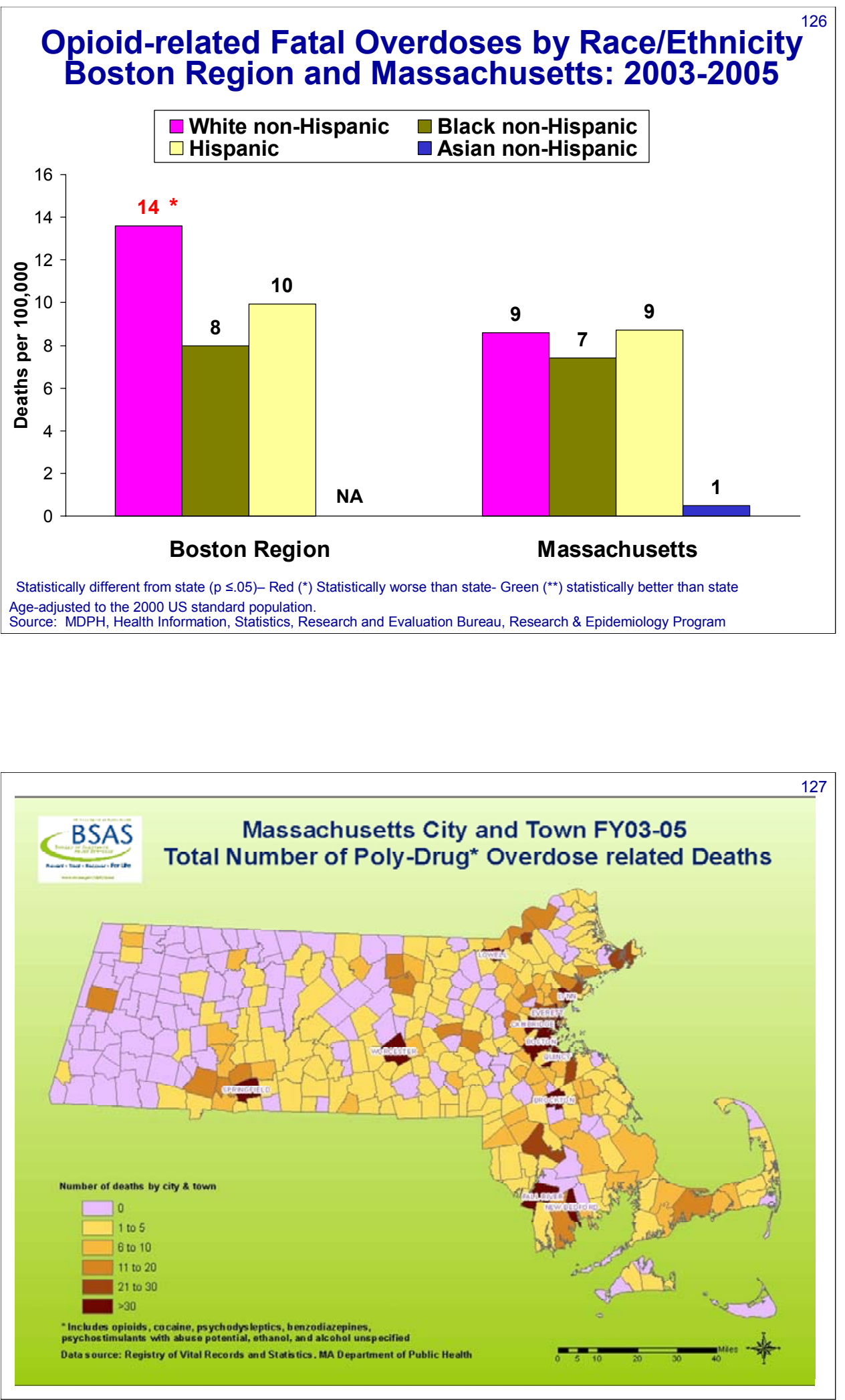


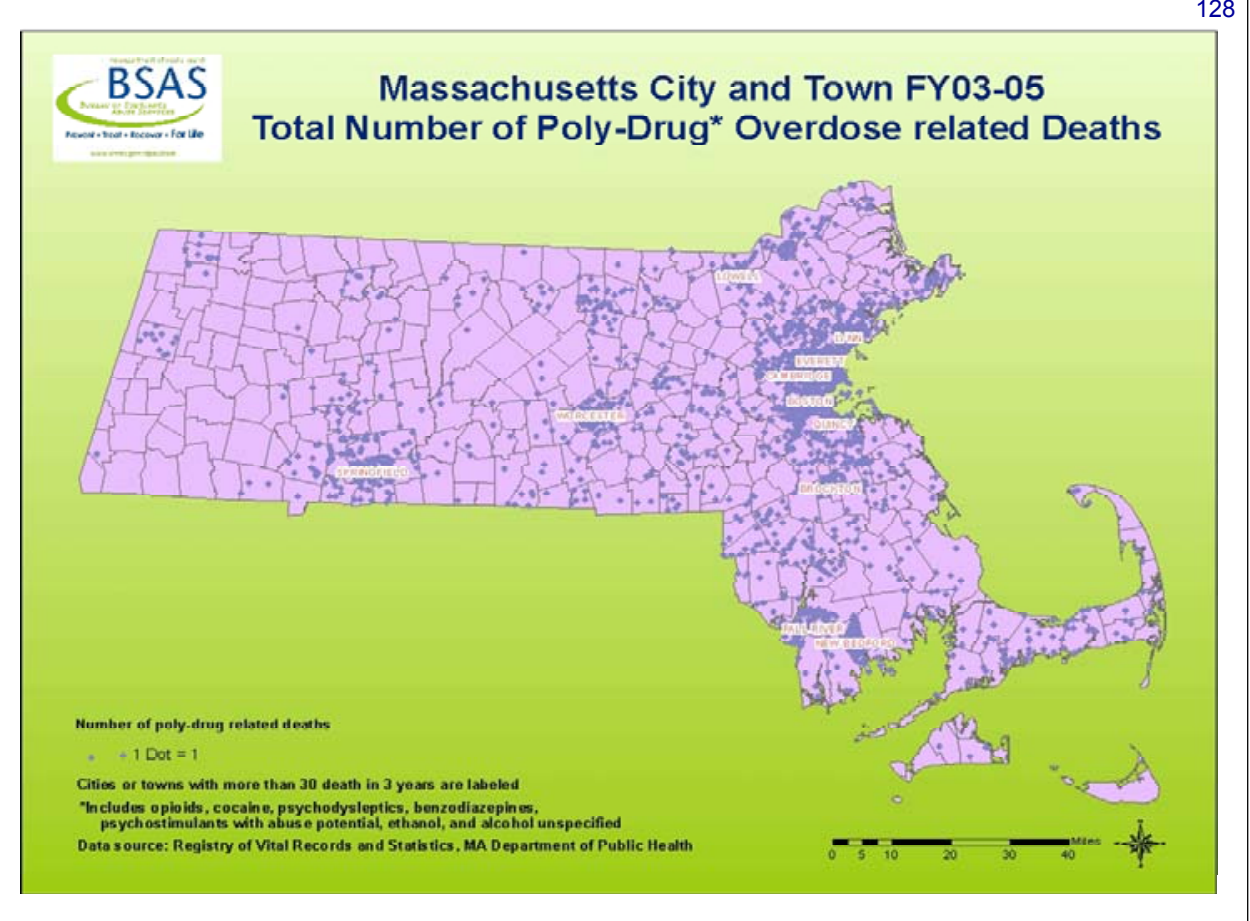

\section{Summary Substance Abuse Indicators Percentages by EOHHS Region}

\begin{tabular}{|c|c|c|c|c|c|c|c|}
\hline Indicator & $\begin{array}{c}\text { MA } \\
\text { Total }\end{array}$ & Western & Central & Northeast & $\begin{array}{l}\text { Metro } \\
\text { West }\end{array}$ & $\begin{array}{c}\text { South } \\
\text { East }\end{array}$ & Boston \\
\hline $\begin{array}{c}\text { Past Month Alcohol } \\
12-20 \text { years }\end{array}$ & 34.1 & 36.7 & 31.2 & 33.5 & 35.3 & 34.0 & 33.6 \\
\hline $\begin{array}{c}\text { Past Month Binge } \\
\text { Drinking- } 12-20 \text { years }\end{array}$ & 24.1 & 27.2 & 24.6 & 22.6 & 24.7 & 24.1 & 21.0 \\
\hline $\begin{array}{l}\text { Any Illicit Drug Use } \\
\text { Past Month, Ages 12+ }\end{array}$ & 9.4 & 10.4 & 8.5 & 8.4 & 8.7 & 9.4 & 12.2 \\
\hline $\begin{array}{l}\text { Any Illicit Drug Use } \\
\text { Other than Marijuana } \\
\text { Past Month, Ages 12+ }\end{array}$ & 3.7 & 3.8 & 3.4 & 3.3 & 3.6 & 3.6 & 4.5 \\
\hline $\begin{array}{c}\text { Alcohol Dependence } \\
\text { or Abuse past Year, } \\
\text { Ages 12+ }\end{array}$ & 8.3 & 8.6 & 8.0 & 7.6 & 8.7 & 7.9 & 9.7 \\
\hline $\begin{array}{l}\text { Dependence or Abuse } \\
\text { of Alcohol or Any } \\
\text { Illicit Drug, Ages 12+ }\end{array}$ & 10.3 & 10.6 & 10.0 & 9.7 & 10.2 & 9.7 & 12.1 \\
\hline
\end{tabular}




\section{Summary Substance Abuse Indicators by EOHHS Region, Massachusetts: 2005}

\begin{tabular}{|c|c|c|c|c|c|c|c|}
\hline Indicator & $\begin{array}{l}\text { MA } \\
\text { Total }\end{array}$ & Western & Central & $\begin{array}{c}\text { North } \\
\text { east }\end{array}$ & $\begin{array}{l}\text { Metro } \\
\text { West }\end{array}$ & $\begin{array}{l}\text { South } \\
\text { East }\end{array}$ & Boston \\
\hline$\%$ Adults Binge Drinking & 15.7 & 16.4 & 15.8 & 16.0 & 14.5 & 15.3 & 18.1 \\
\hline$\%$ Adults Smoking & 18.1 & 18.8 & 21.2 & 18.3 & 12.4 & 20.4 & 19.5 \\
\hline$\%$ Adults using illicit drugs & 8.1 & 13.9 & 7.2 & 7.2 & 7.0 & 8.1 & 5.3 \\
\hline $\begin{array}{l}\text { Substance Abuse Treatment } \\
\text { Program Admissions Rate }\end{array}$ & 1,601 & 2,044 & 1,441 & 1,379 & 871 & 1,724 & 2,978 \\
\hline $\begin{array}{c}\text { Substance Abuse Treatment } \\
\text { Program Admissions Rate-- } \\
\text { Alcohol }\end{array}$ & 661 & 921 & 571 & 498 & 404 & 821 & 1,010 \\
\hline $\begin{array}{c}\text { Substance Abuse Treatment } \\
\text { Program Admissions Rate- } \\
\text { Cocaine }\end{array}$ & 62 & 65 & 44 & 58 & 34 & 88 & 99 \\
\hline $\begin{array}{c}\text { Substance Abuse Treatment } \\
\text { Program Admissions Rate- } \\
\text { Heroin }\end{array}$ & 609 & 635 & 636 & 583 & 286 & 504 & 1,453 \\
\hline
\end{tabular}

\begin{tabular}{|c|c|c|c|c|c|c|c|}
\hline \multicolumn{8}{|c|}{$\begin{array}{c}\text { Summary Opioid Indicators by EOHHS Region } \\
\text { Massachusetts: 2003-2005 }\end{array}$} \\
\hline Indicator & $\begin{array}{c}\text { MA } \\
\text { Total }\end{array}$ & Western & Central & Northeast & $\begin{array}{l}\text { Metro } \\
\text { West }\end{array}$ & $\begin{array}{c}\text { South } \\
\text { East }\end{array}$ & Boston \\
\hline $\begin{array}{c}\text { Opioid- } \\
\text { related } \\
\text { Emergency } \\
\text { Department } \\
\text { Visit Rate }\end{array}$ & 181 & 114 & 169 & 223 & 126 & 196 & 309 \\
\hline $\begin{array}{l}\text { Opioid- } \\
\text { related fatal } \\
\text { overdose } \\
\text { rate }\end{array}$ & 8.1 & 6.7 & 6.5 & 9.5 & 6.0 & 10.1 & 10.8 \\
\hline$\square$ Bet & utcome & icantly differe & t from state) & Worse Outcor & me (significa & tly different $f$ & m state) \\
\hline
\end{tabular}




\section{INJURY AND VIOLENCE}


Causes of Injury Deaths Boston Region: 2005
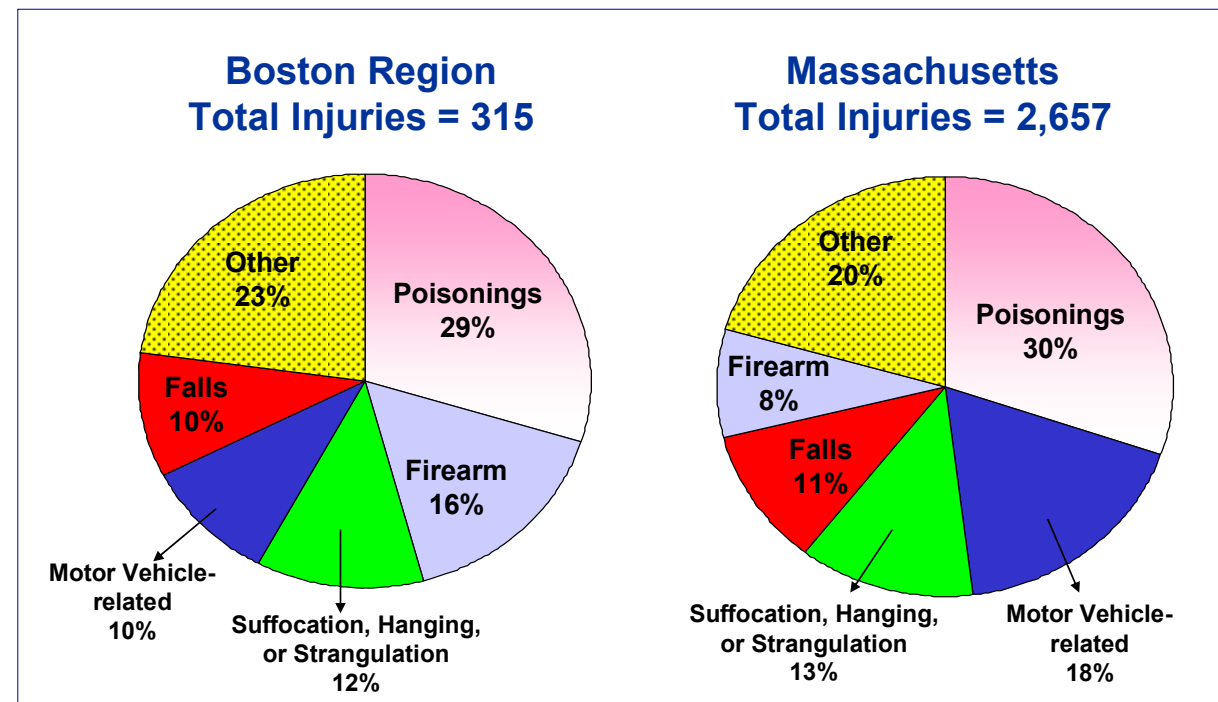

Source: Massachusetts Department of Public Health, Center for Health Information, Statistics, Research, and Evaluation

\section{Emergency Department Visit Rates for Injuries \& Poisonings by EOHHS Region Massachusetts: 2003-2005}

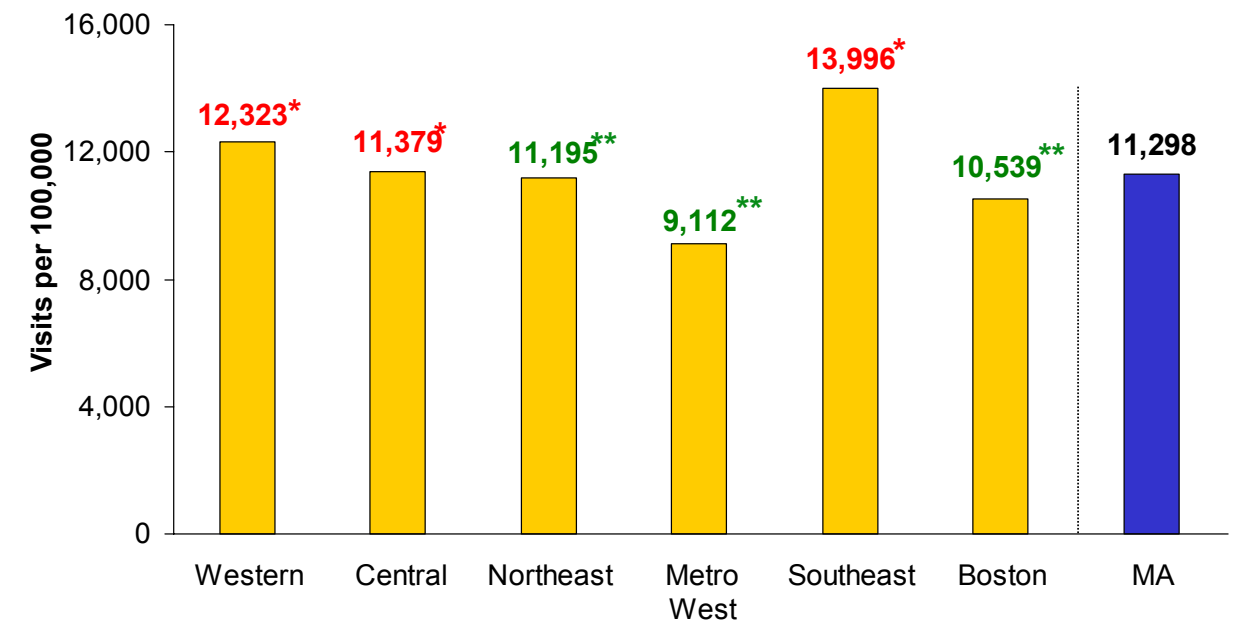

Statistically different from state $(p \leq .05)-\operatorname{Red}\left({ }^{*}\right)$ Statistically worse than state- Green $\left(^{* *}\right)$ statistically better than state Age-adjusted to the 2000 US standard population.

Source: Division of Health Care Finance and Policy. Calendar Year 2003- 2005. Emergency Department Visits 


\section{Injury-Related Hospital Discharge Rates by EOHHS Region Massachusetts: 2003-2005}

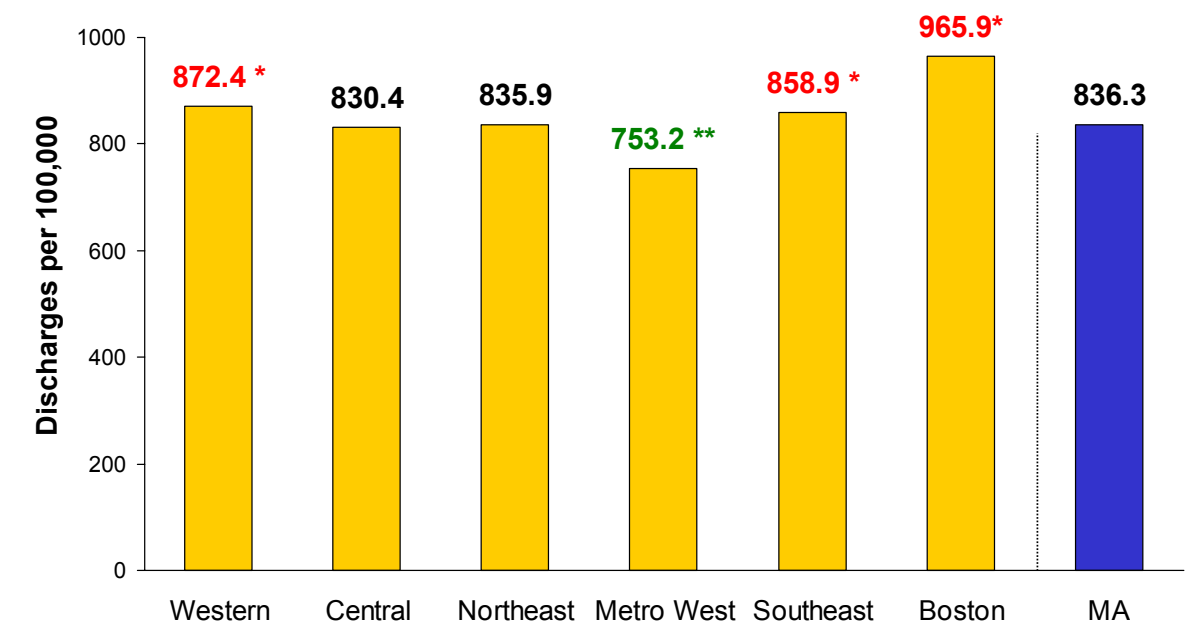

Statistically different from state $(p \leq .05)-$ Red $\left(^{*}\right)$ Statistically worse than state- Green $\left(^{* *}\right)$ statistically better than state Age-adjusted to the 2000 US standard population.

Source: Division of Health Care Finance and Policy. Calendar Year 2003- 2005. Hospital Discharges

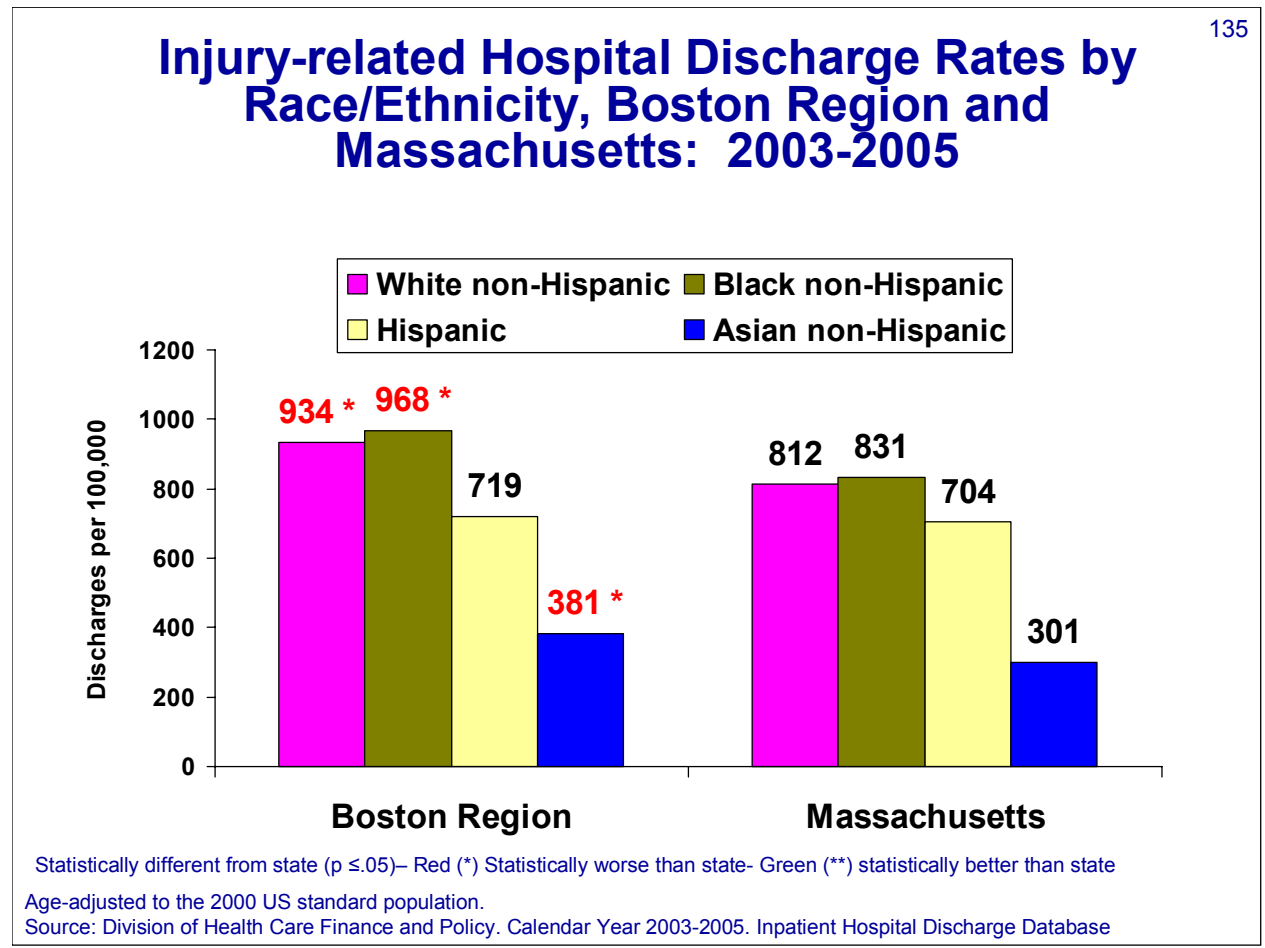




\section{Injury Death Rates by EOHHS Region Massachusetts: 2003-2005}

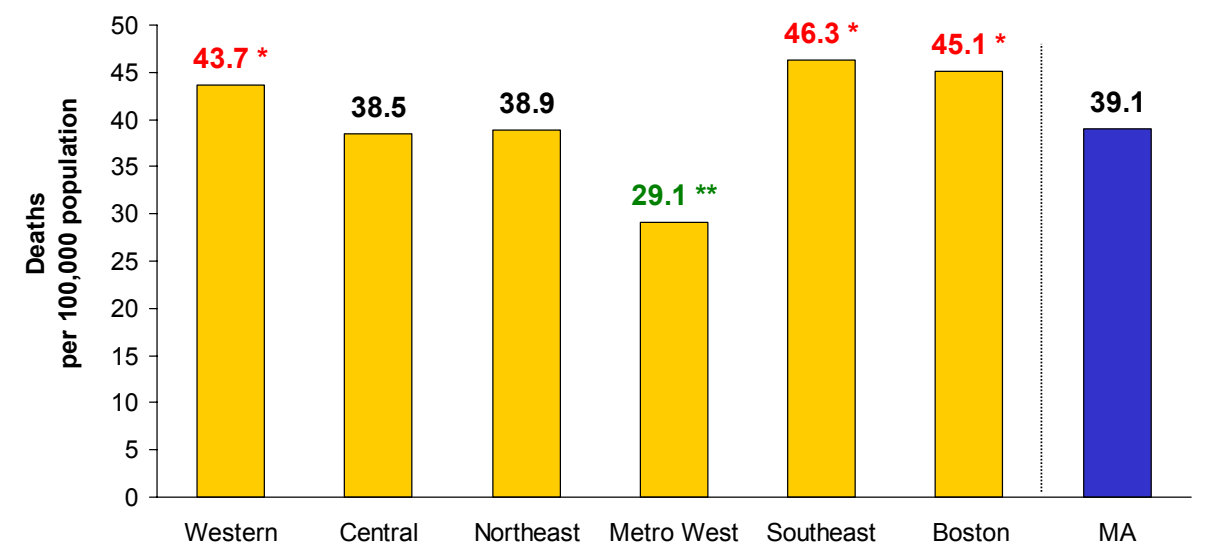

Statistically different from state $(p \leq .05)-\operatorname{Red}\left({ }^{*}\right)$ Statistically worse than state- Green $\left(^{* *}\right)$ statistically better than state

\section{Emergency Department Visit Rates for Injuries \& Poisonings by Race/Ethnicity Boston Region and Massachusetts: 2003-2005}

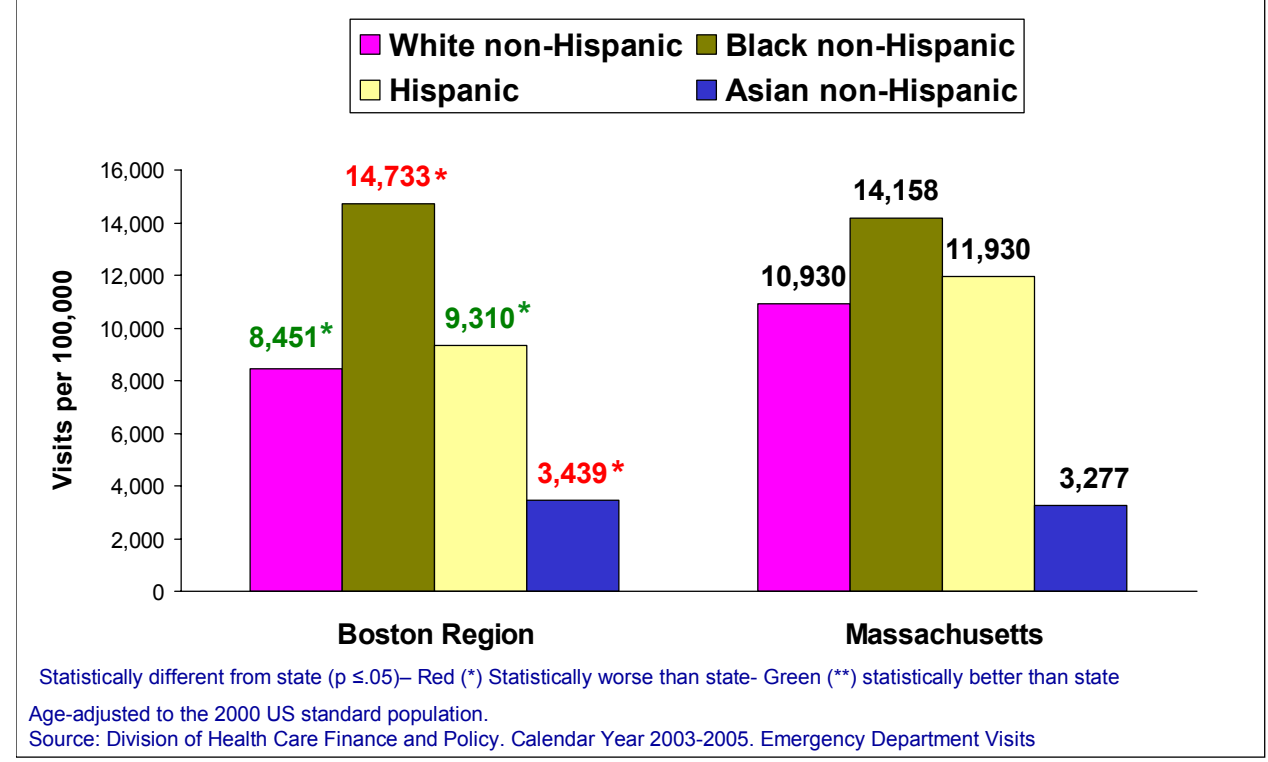




\section{Hospital Discharge Rates for Assaults by EOHHS Region, Massachusetts: 2003-2005}

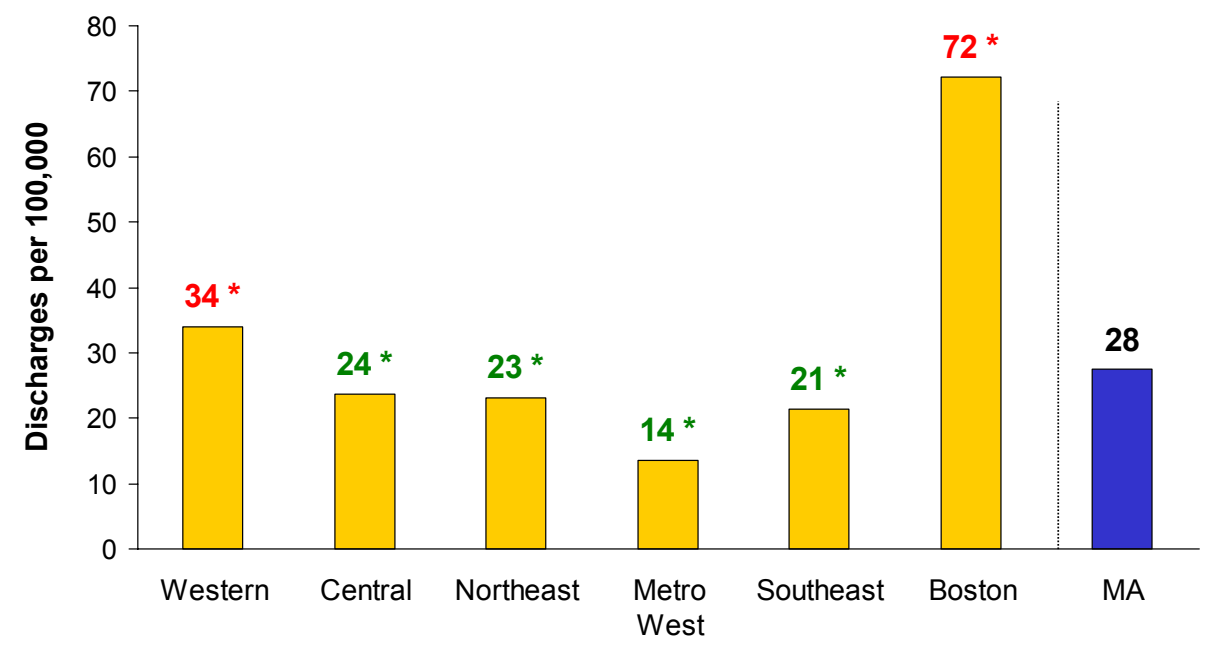

Statistically different from state $(p \leq .05)-\operatorname{Red}\left({ }^{*}\right)$ Statistically worse than state- Green $\left(^{\star *}\right)$ statistically better than state

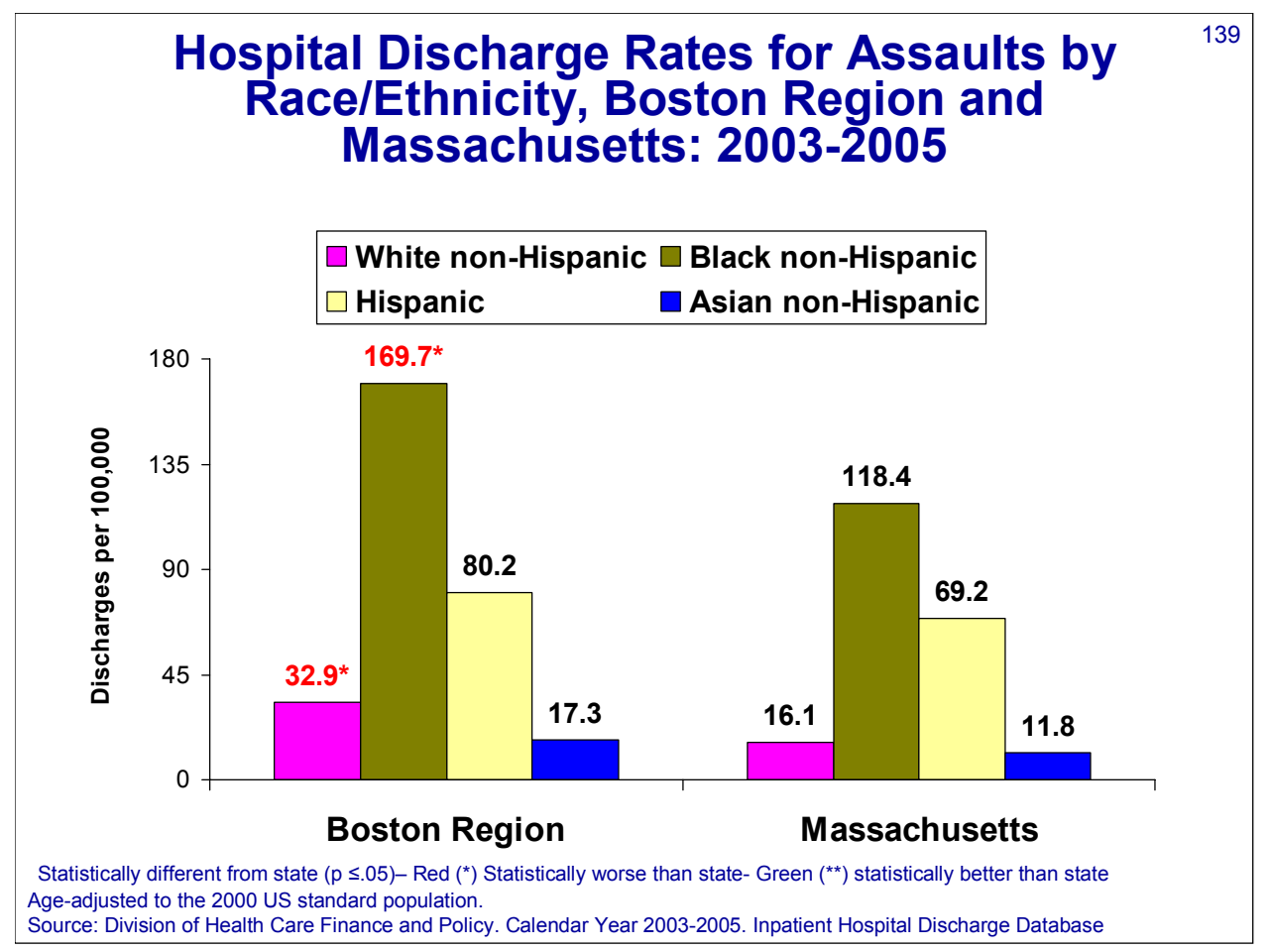




\section{Homicides by EOHHS Region Massachusetts: 2003-2005}

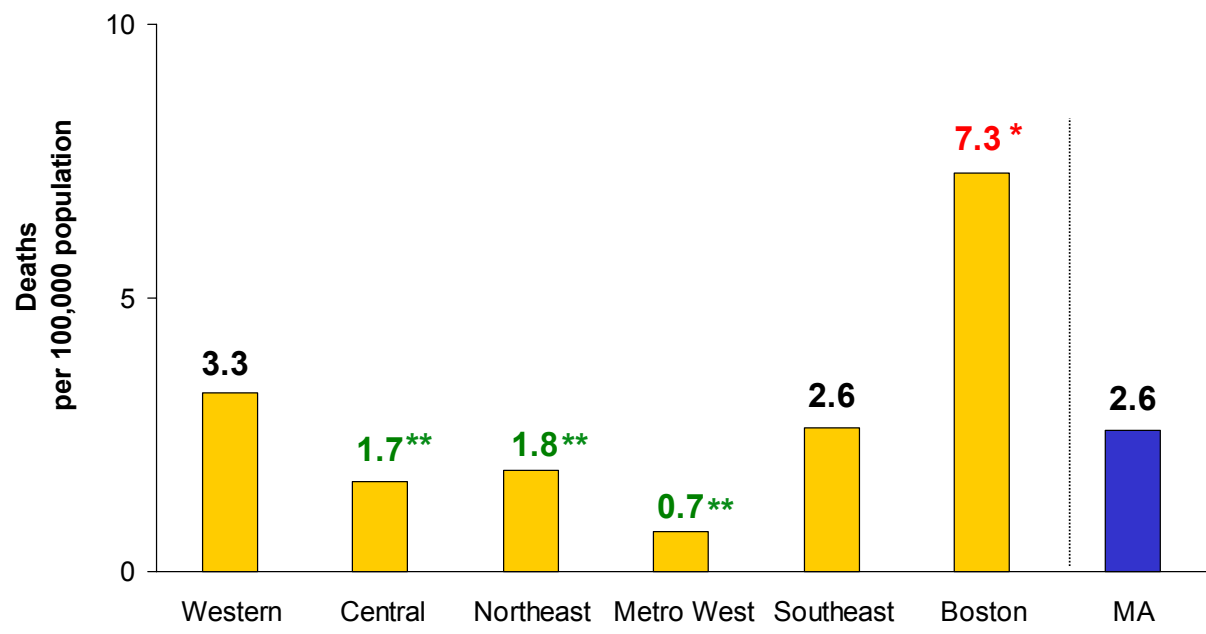

Statistically different from state $(p \leq .05)-\operatorname{Red}\left({ }^{*}\right)$ Statistically worse than state- Green $\left(^{\star \star}\right)$ statistically better than state Age-adjusted to the 2000 US standard population.

Source: MDPH, Health Information, Statistics, Research and Evaluation Bureau, Research \& Epidemiology Program

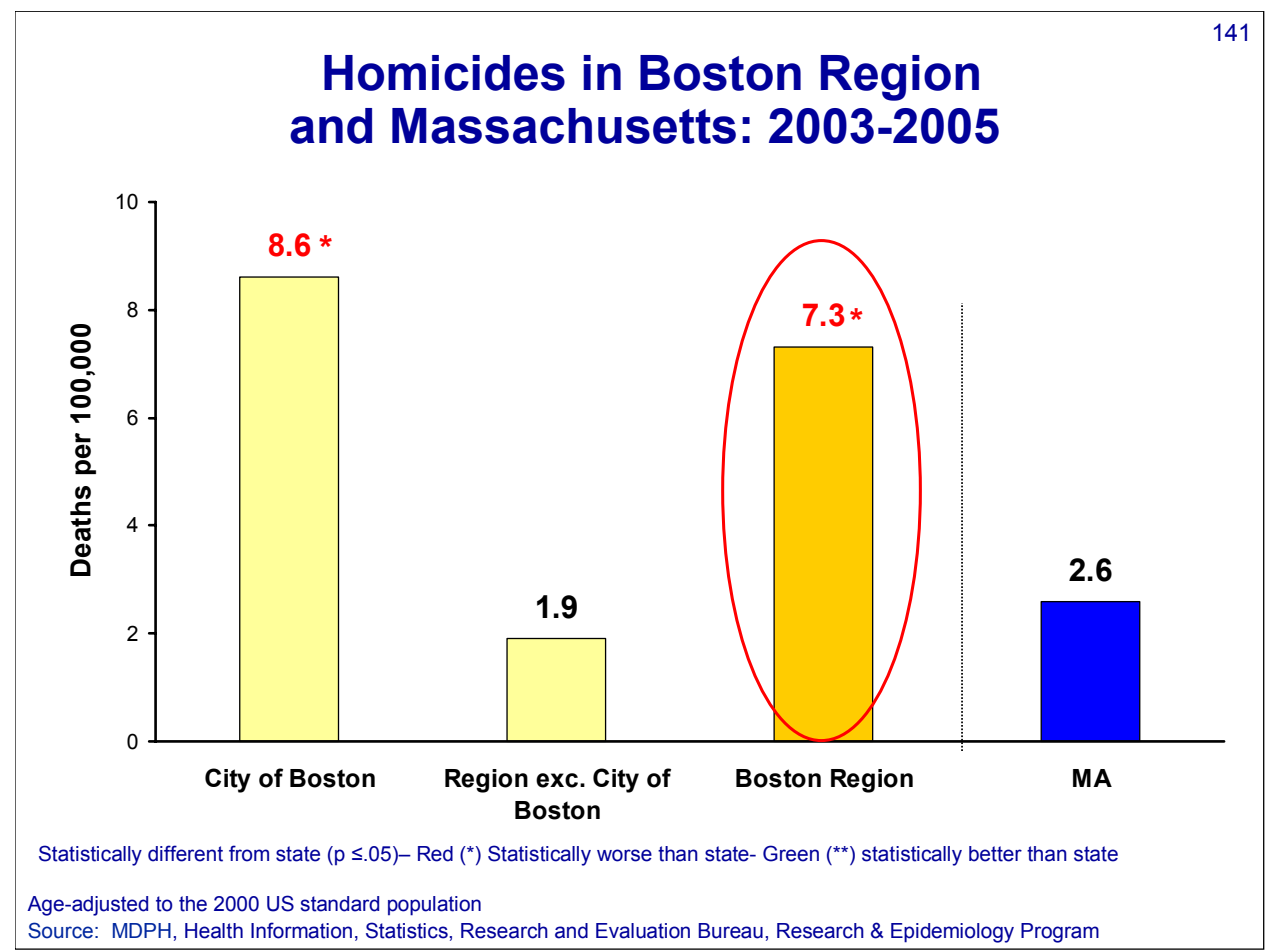




\section{Homicide Rates by Race/Ethnicity Boston Region and Massachusetts: 2003-2005}

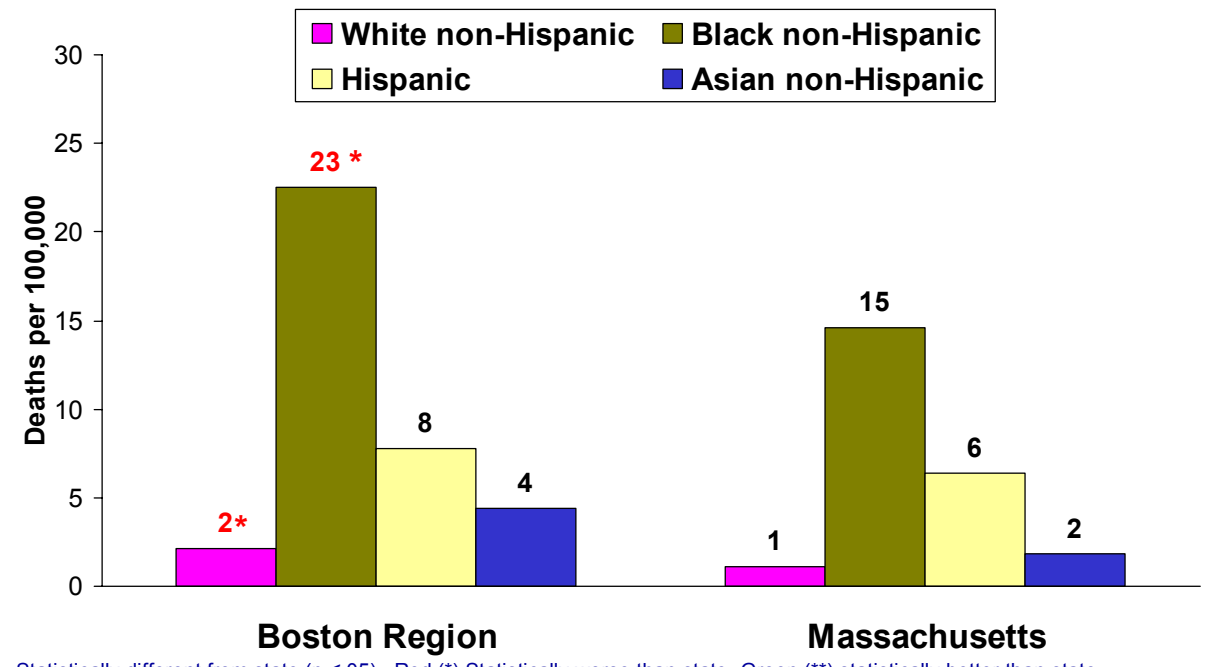

Statistically different from state $(p \leq .05)-\operatorname{Red}\left({ }^{*}\right)$ Statistically worse than state- Green $\left(^{* \star}\right)$ statistically better than state Age-adjusted to the 2000 US standard population.

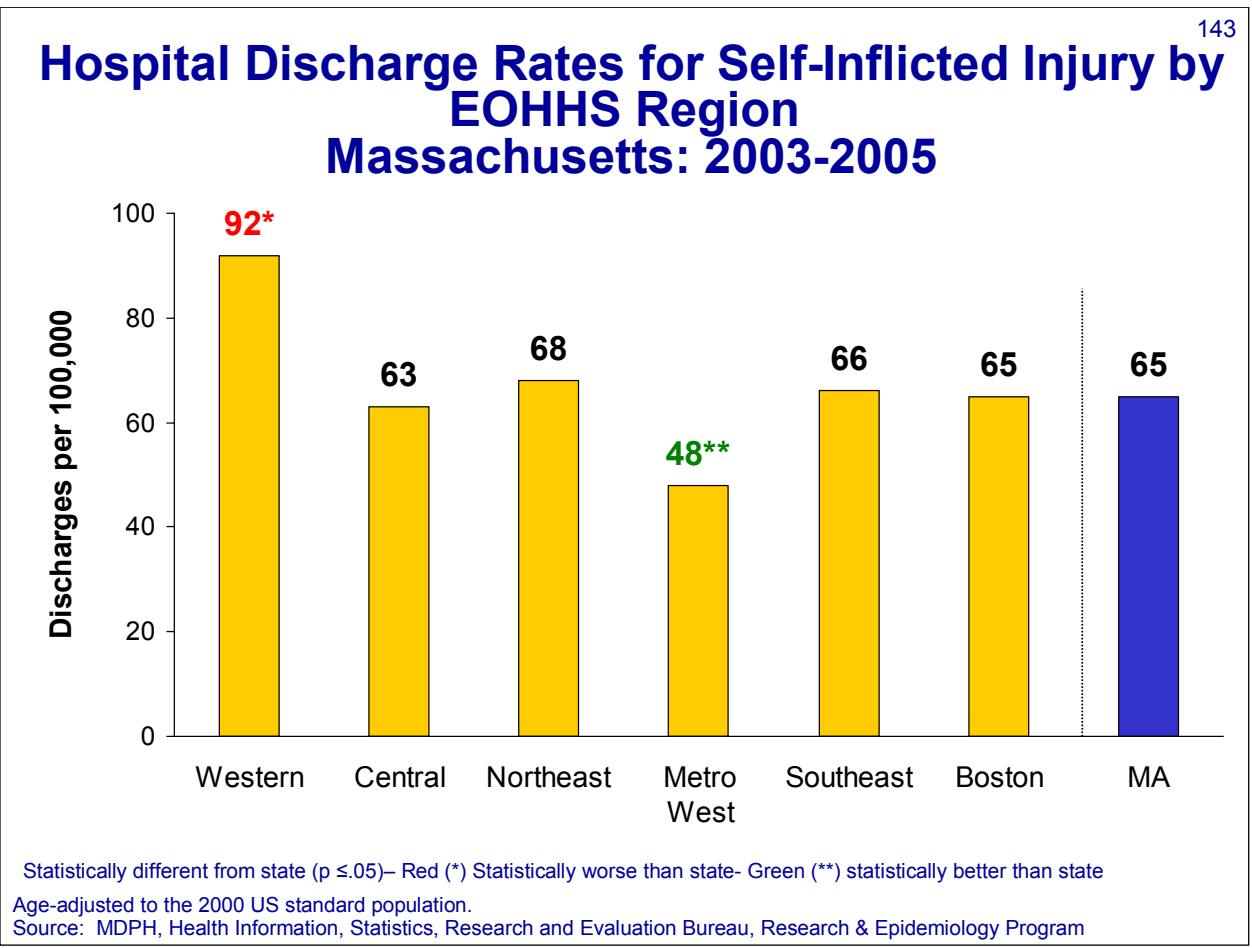


Hospital Discharge Rates for Self-inflicted Injuries by Race/Ethnicity, Boston Region and Massachusetts: 2003-2005

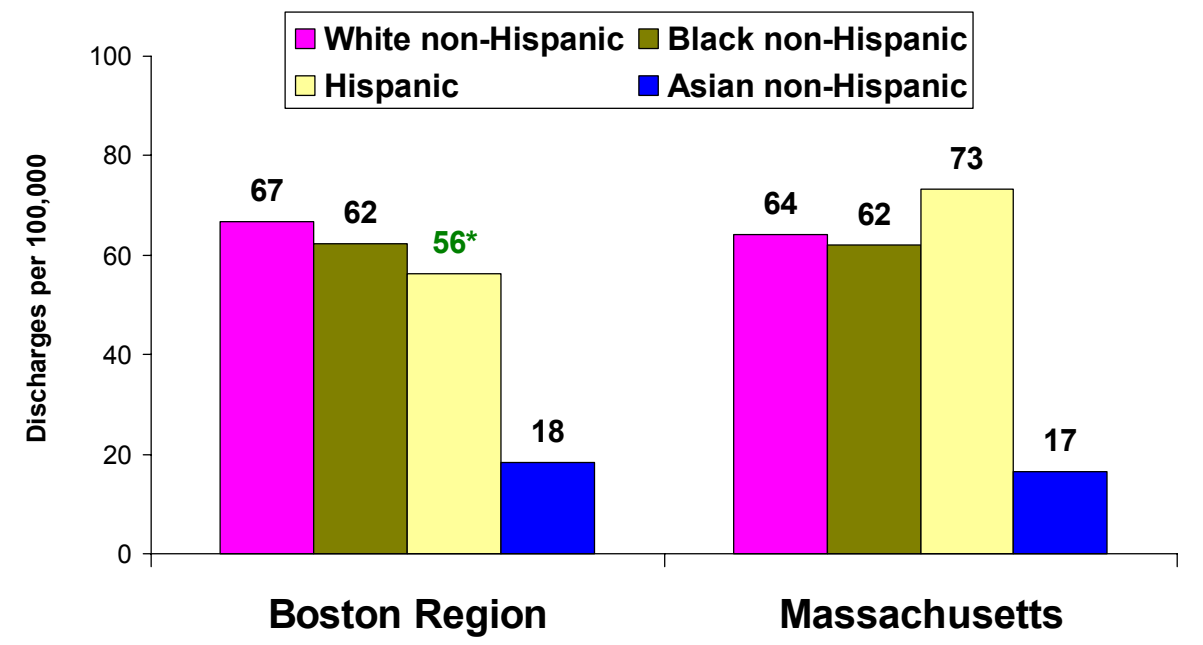

Statistically different from state $(p \leq .05)-\operatorname{Red}\left({ }^{*}\right)$ Statistically worse than state- Green $\left(^{* \star}\right)$ statistically better than state Age-adjusted to the 2000 US standard population.

Source: Division of Health Care Finance and Policy. Calendar Year 2003-2005. Inpatient Hospital Discharge Database

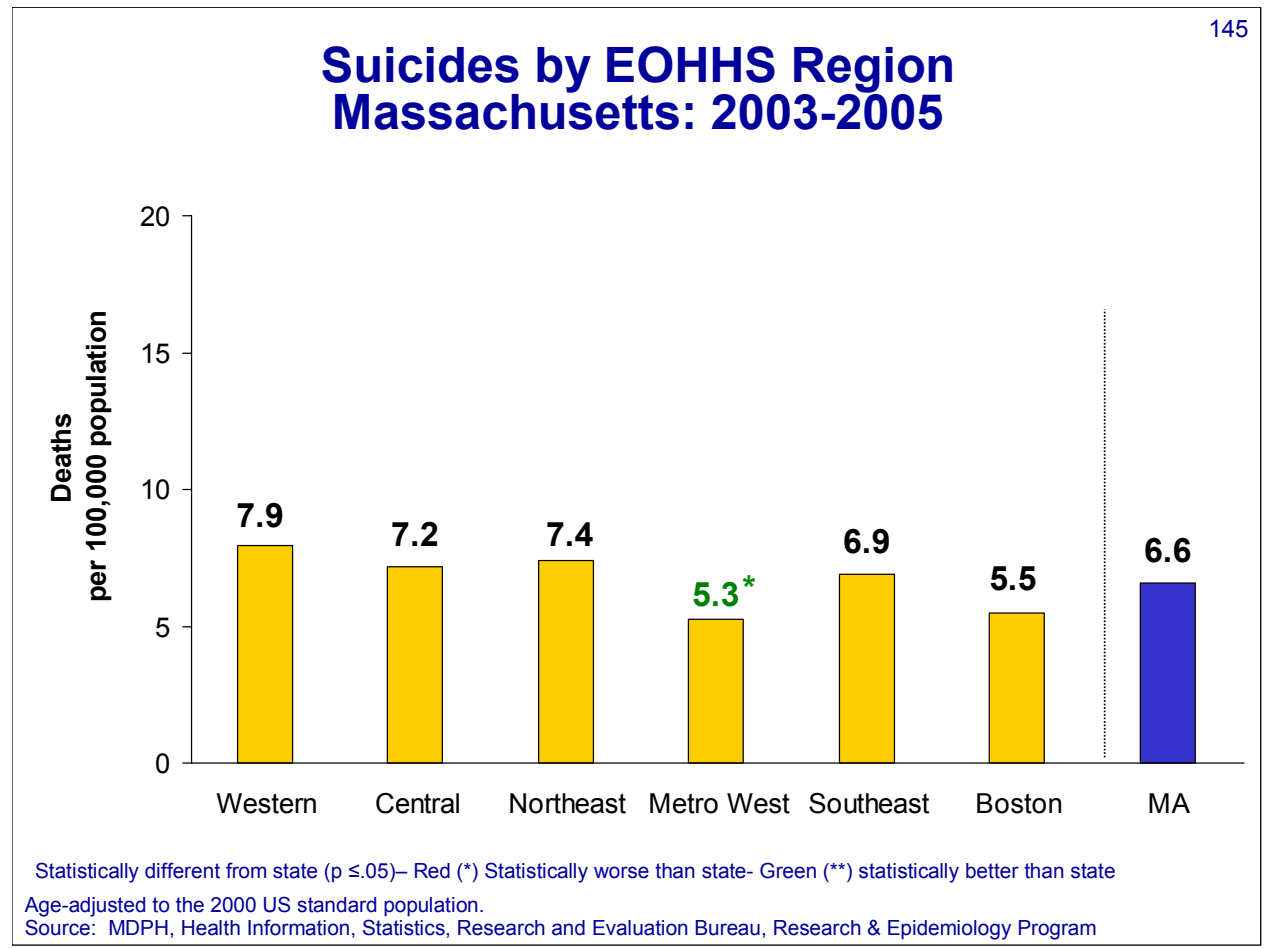


Suicide Rates by Race/Ethnicity

Boston Region and Massachusetts: 2003-2005

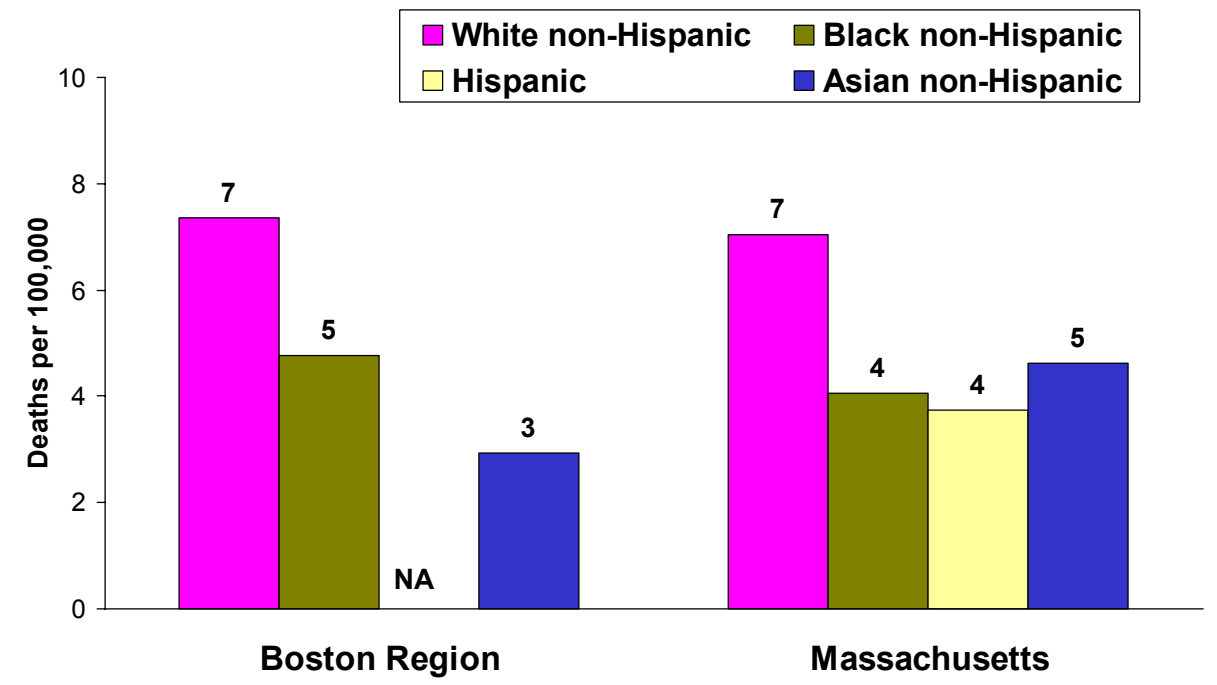

Statistically different from state $(p \leq .05)-\operatorname{Red}\left({ }^{*}\right)$ Statistically worse than state- Green $\left({ }^{* *}\right)$ statistically better than state Age-adjusted to the 2000 US standard population

Source: MDPH, Health Information, Statistics, Research and Evaluation Bureau, Research \& Epidemiology Program

\section{Hospital Discharge Rates for Motor Vehicle-related Injuries by EOHHS Region Massachusetts: 2003-2005}

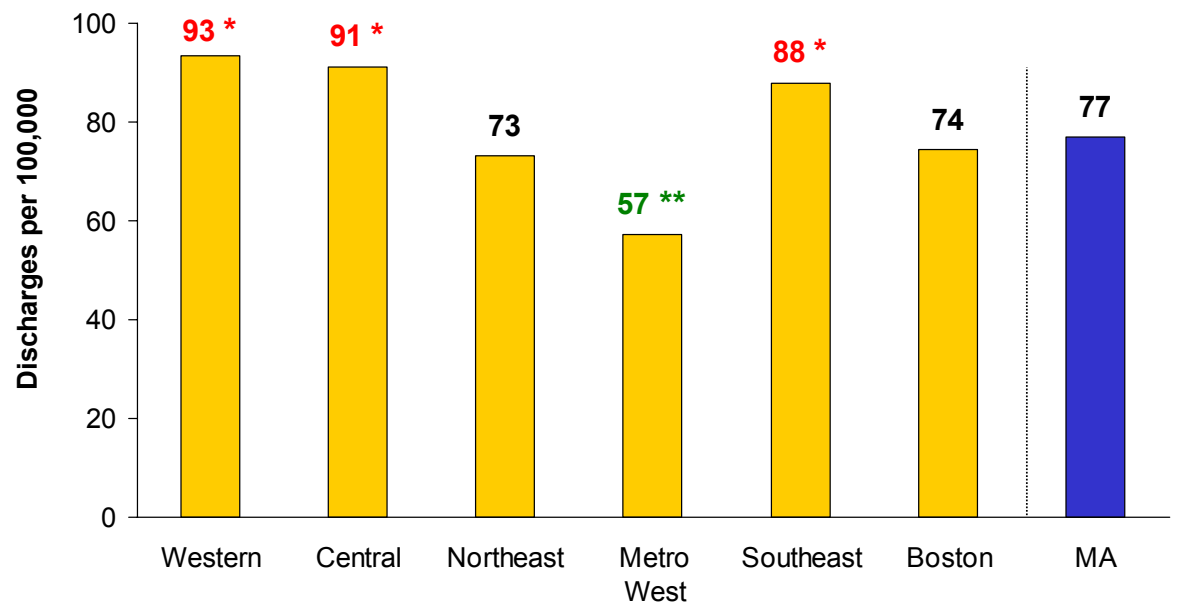

Statistically different from state $(p \leq .05)-\operatorname{Red}\left({ }^{*}\right)$ Statistically worse than state- Green $\left(^{\star \star}\right)$ statistically better than state Age-adjusted to the 2000 US standard population.

Source: MDPH, Health Information, Statistics, Research and Evaluation Bureau, Research \& Epidemiology Program 
Hospital Discharge Rates for Motor Vehicle-related Injuries by Race/Ethnicity

Boston Region and Massachusetts: 2003-2005
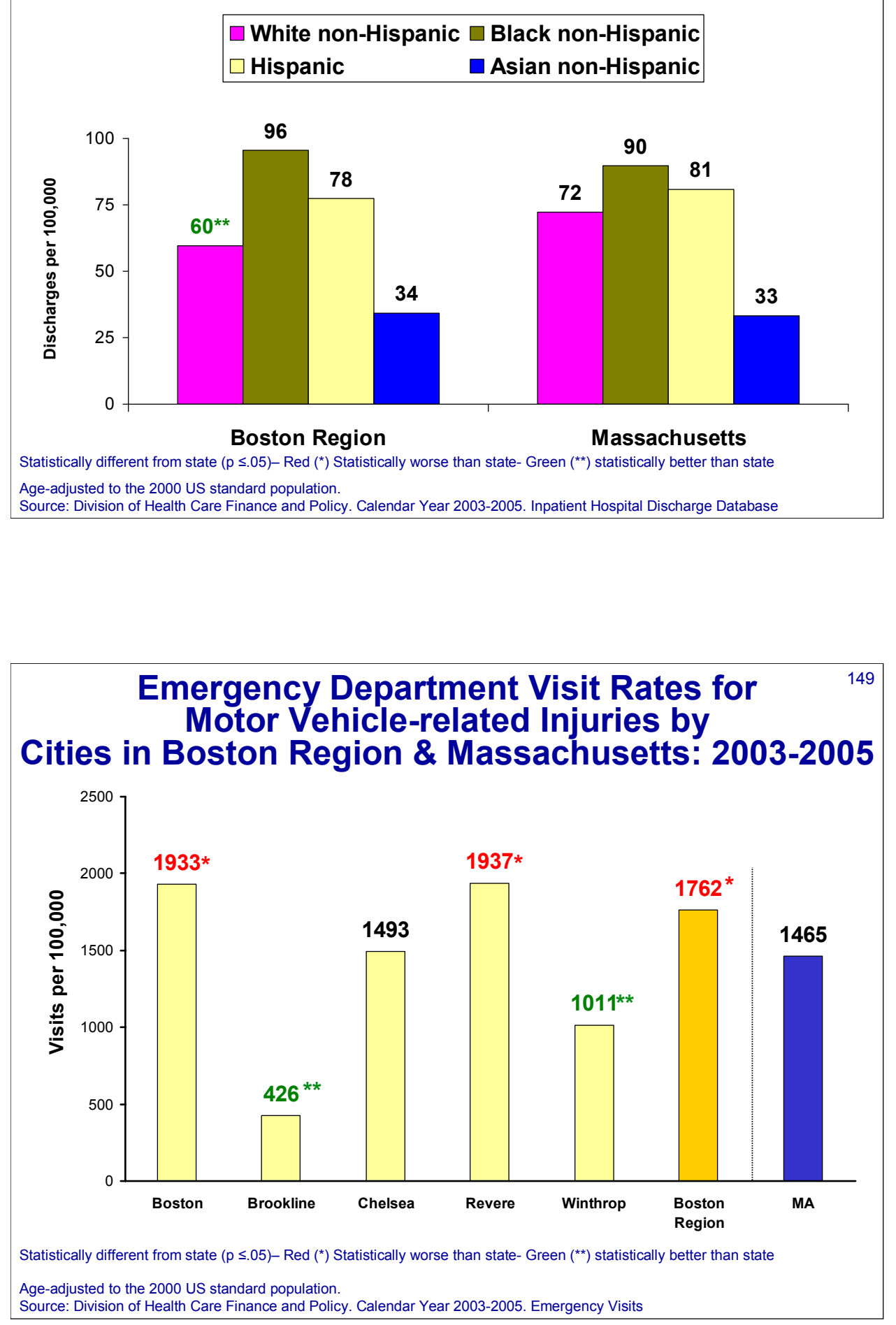


\section{Motor Vehicle-related Death Rates by EOHHS Region \\ Massachusetts: 2003-2005}

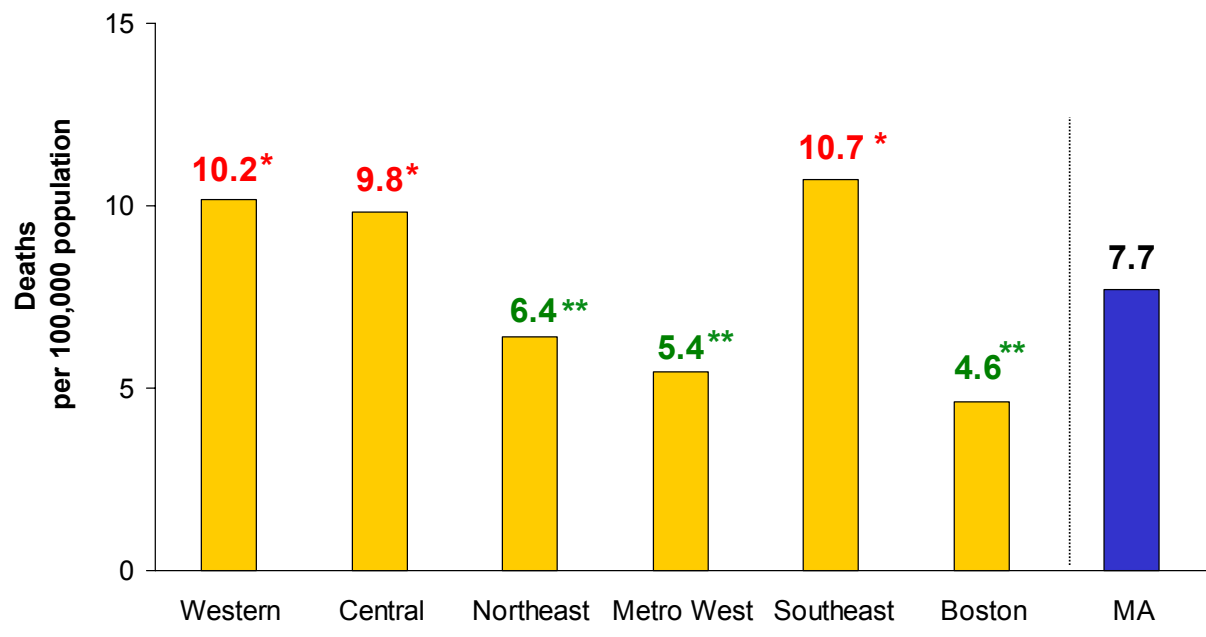

Statistically different from state $(p \leq .05)-\operatorname{Red}\left({ }^{*}\right)$ Statistically worse than state- Green $\left(^{\star \star}\right)$ statistically better than state

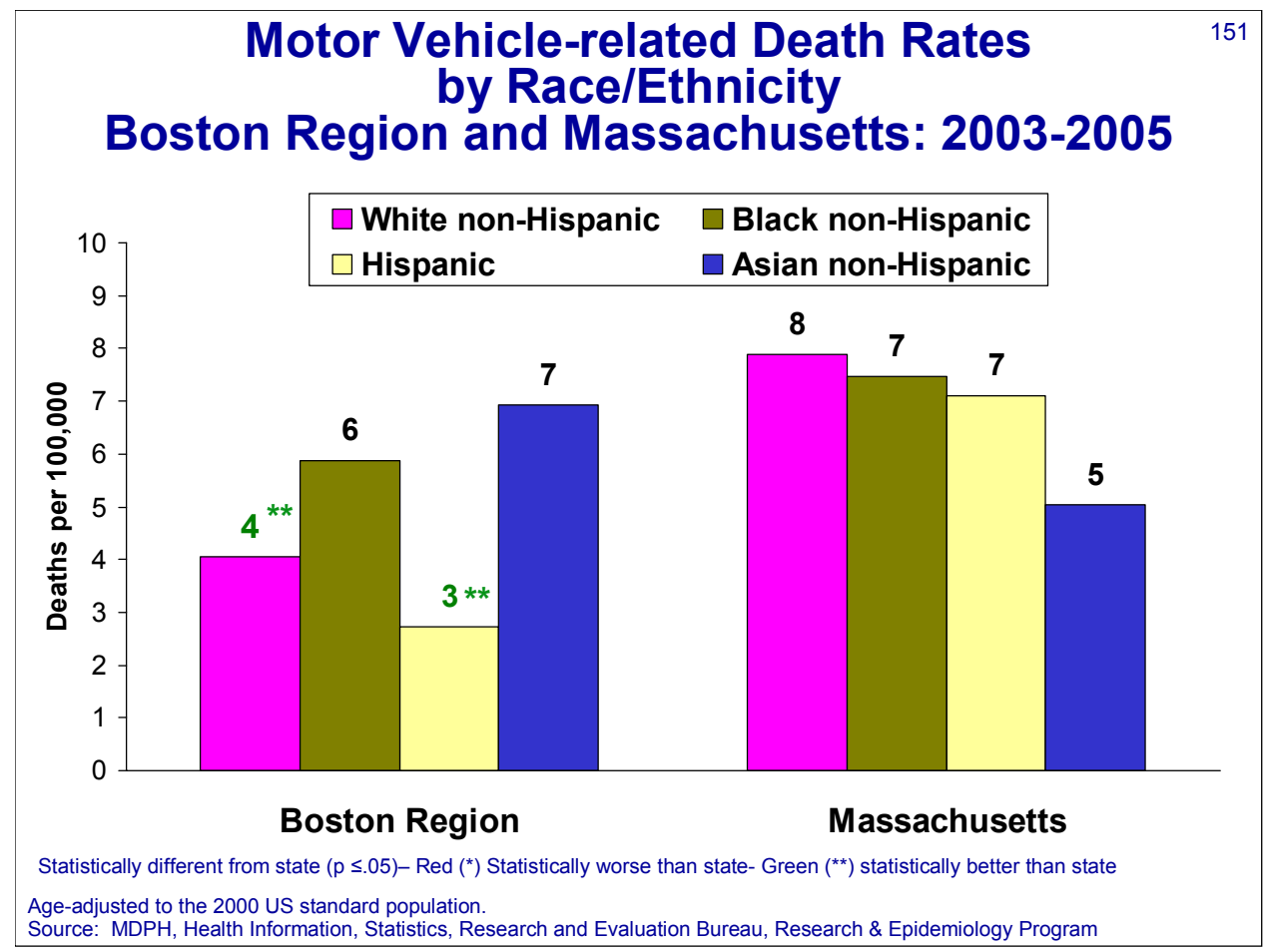


Emergency Department Visit Rates for Traumatic Brain Injury by EOHHS Region Massachusetts: 2003-2005

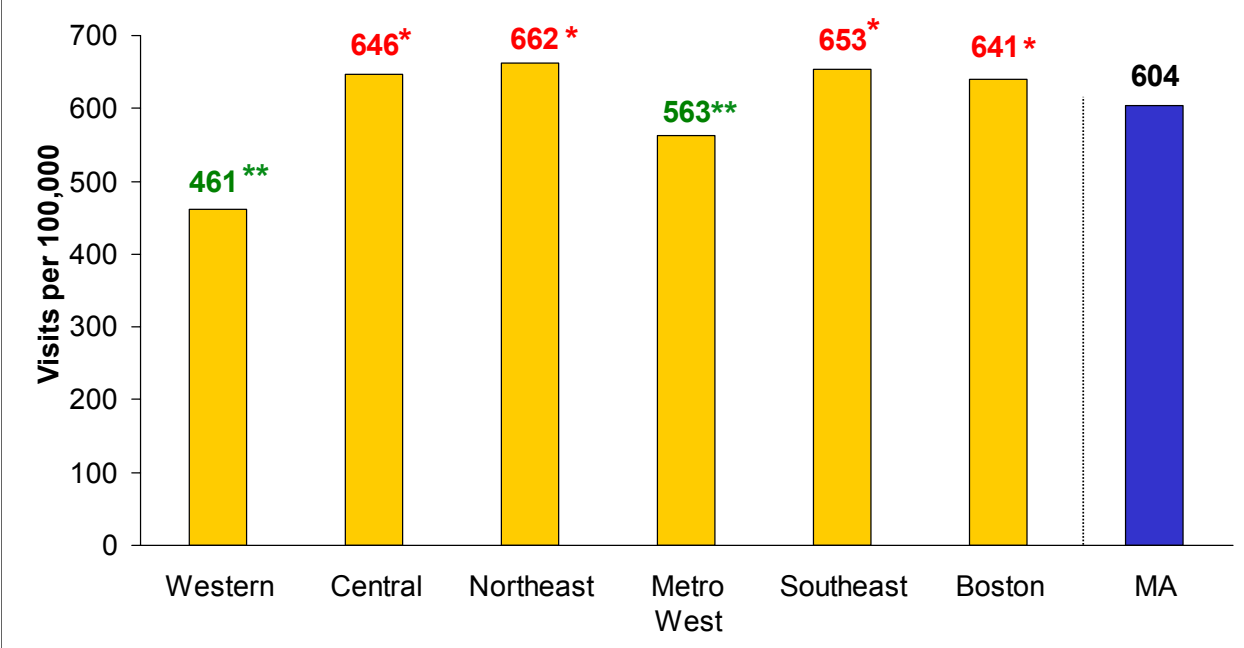

Statistically different from state $(p \leq .05)-\operatorname{Red}\left({ }^{*}\right)$ Statistically worse than state- Green $\left(^{* \star}\right)$ statistically better than state Age-adjusted to the 2000 US standard population.

Source: Division of Health Care Finance and Policy. Calendar Year 2005. Emergency Visits

\section{Emergency Department Visit Rates for Traumatic Brain Injury by Race/Ethnicity Boston Region and Massachusetts: 2003-2005}

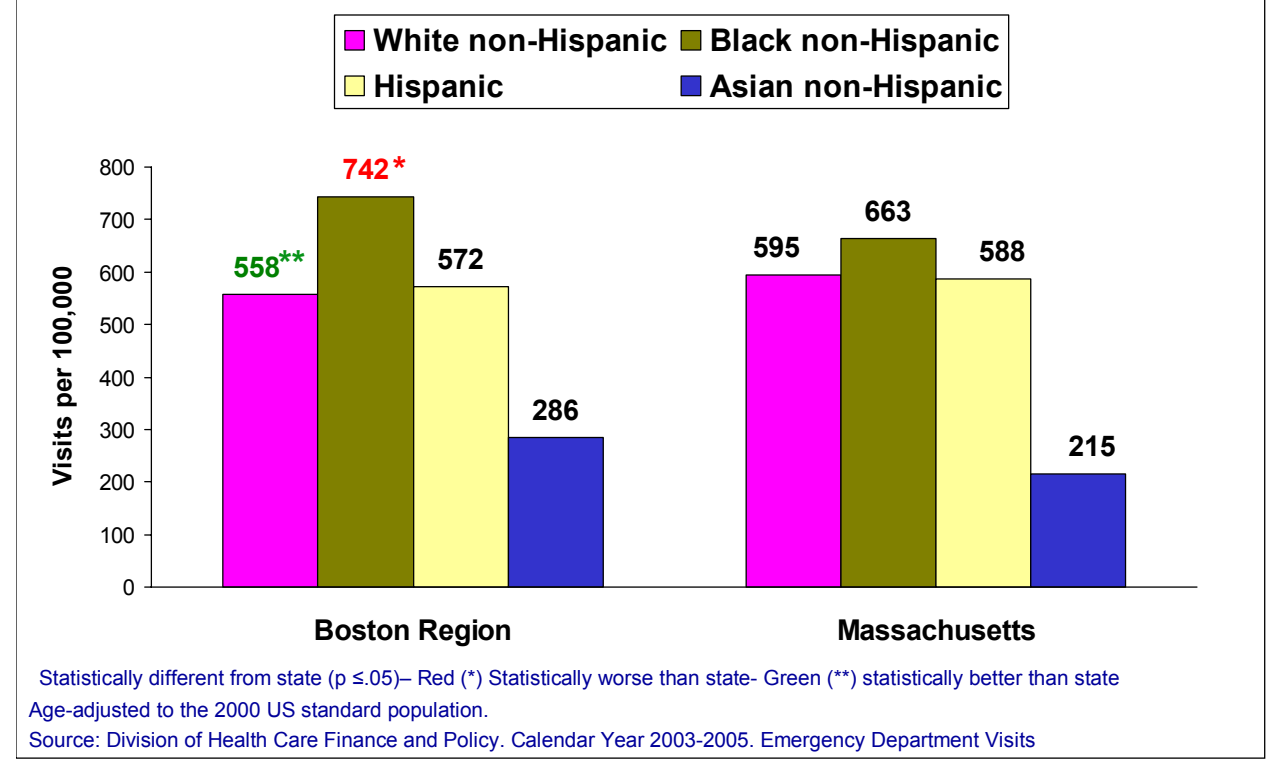


Emergency Visit Rates

for Traumatic Brain Injury by Cities

in Boston Region \& Massachusetts: 2003-2005

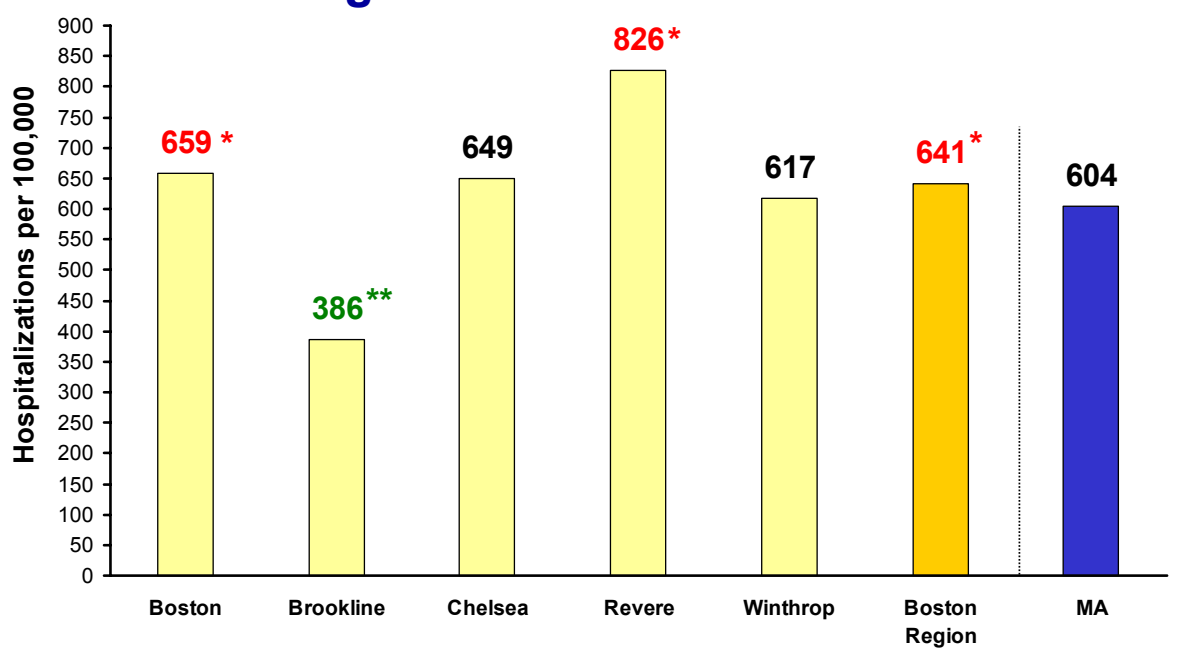

Statistically different from state $(p \leq .05)-\operatorname{Red}\left({ }^{*}\right)$ Statistically worse than state- Green $\left(^{* *}\right)$ statistically better than state

Age-adjusted to the 2000 US standard population.

Source: Division of Health Care Finance and Policy. Calendar Year 2003-2005. Inpatient Hospital Discharge Database

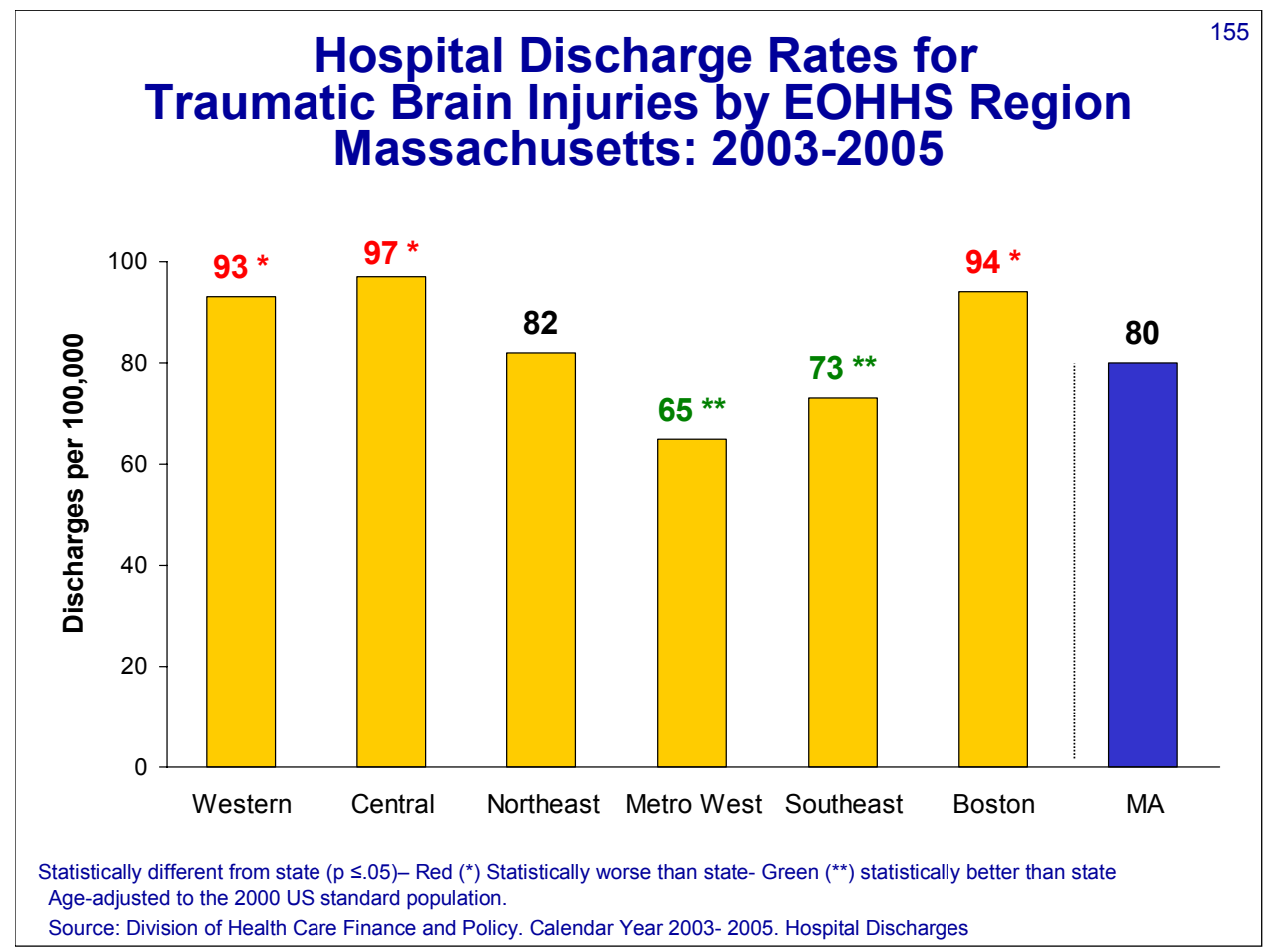



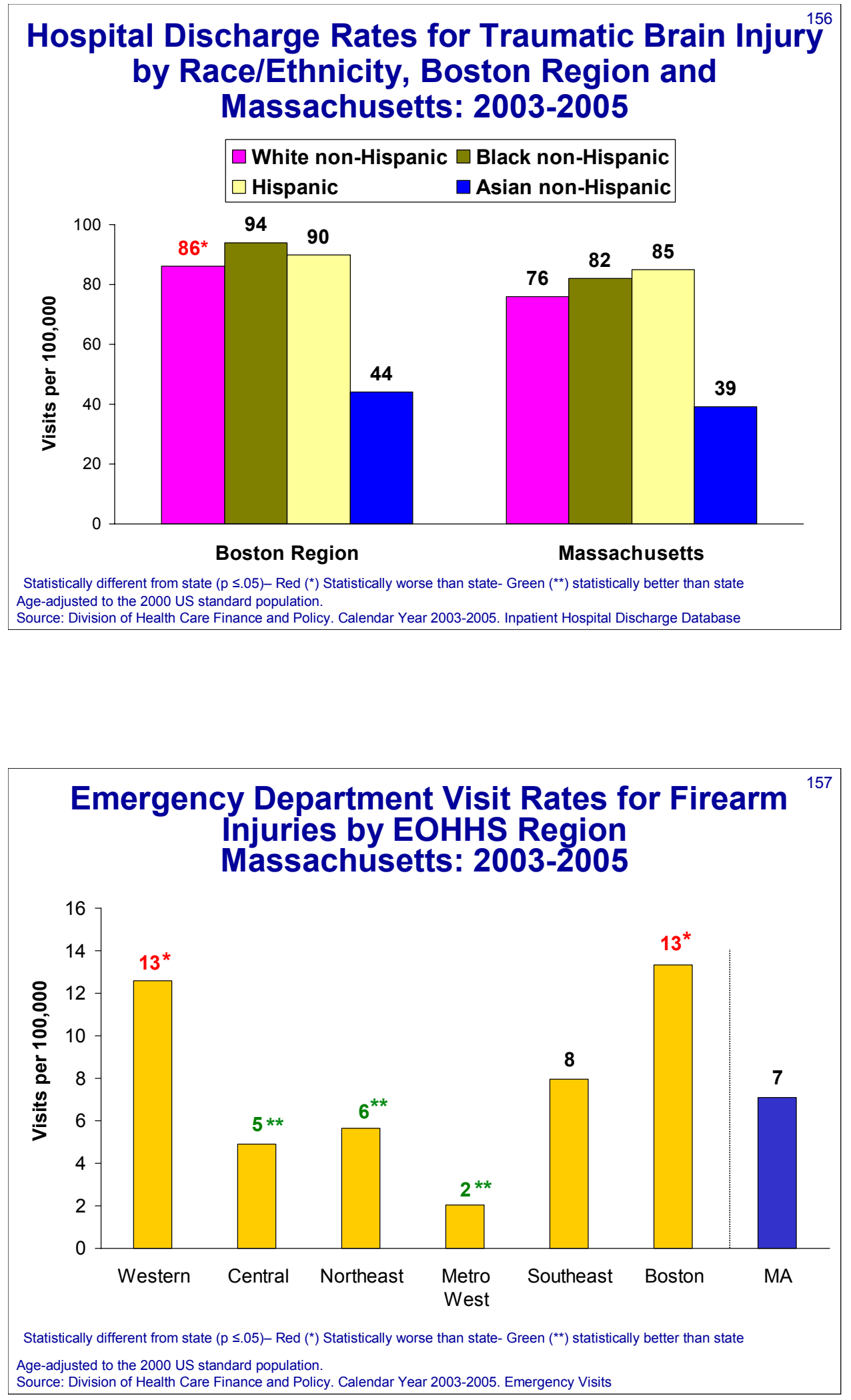


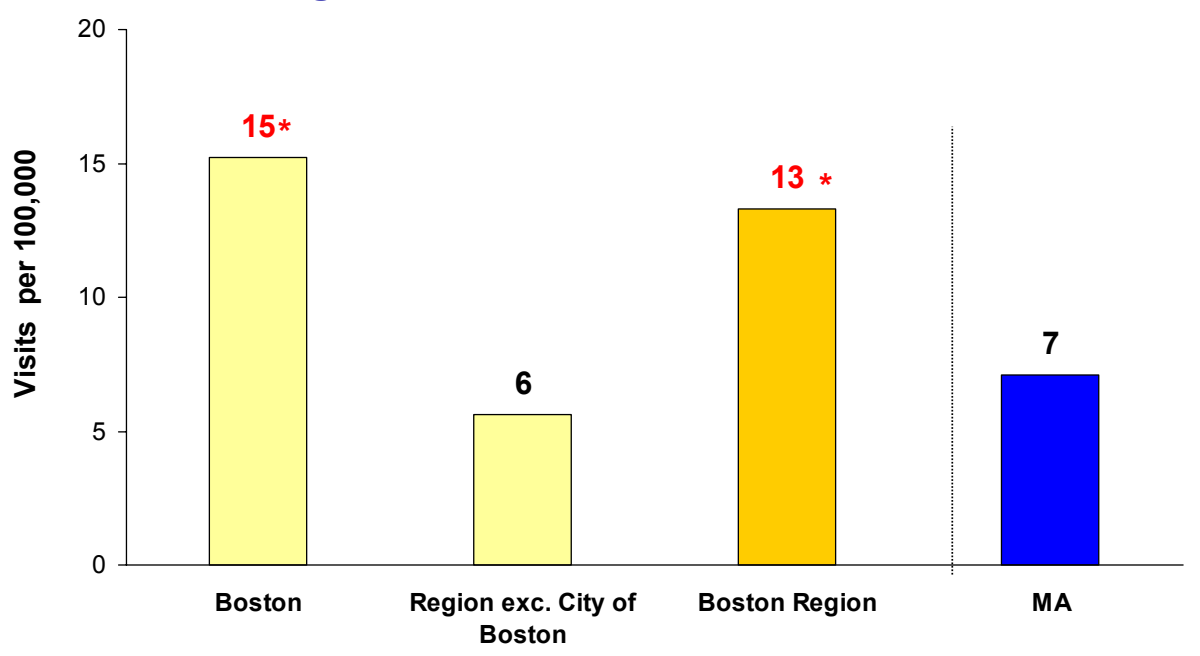

Statistically different from state $(p \leq .05)-\operatorname{Red}\left({ }^{*}\right)$ Statistically worse than state- Green $\left(^{* *}\right)$ statistically better than state

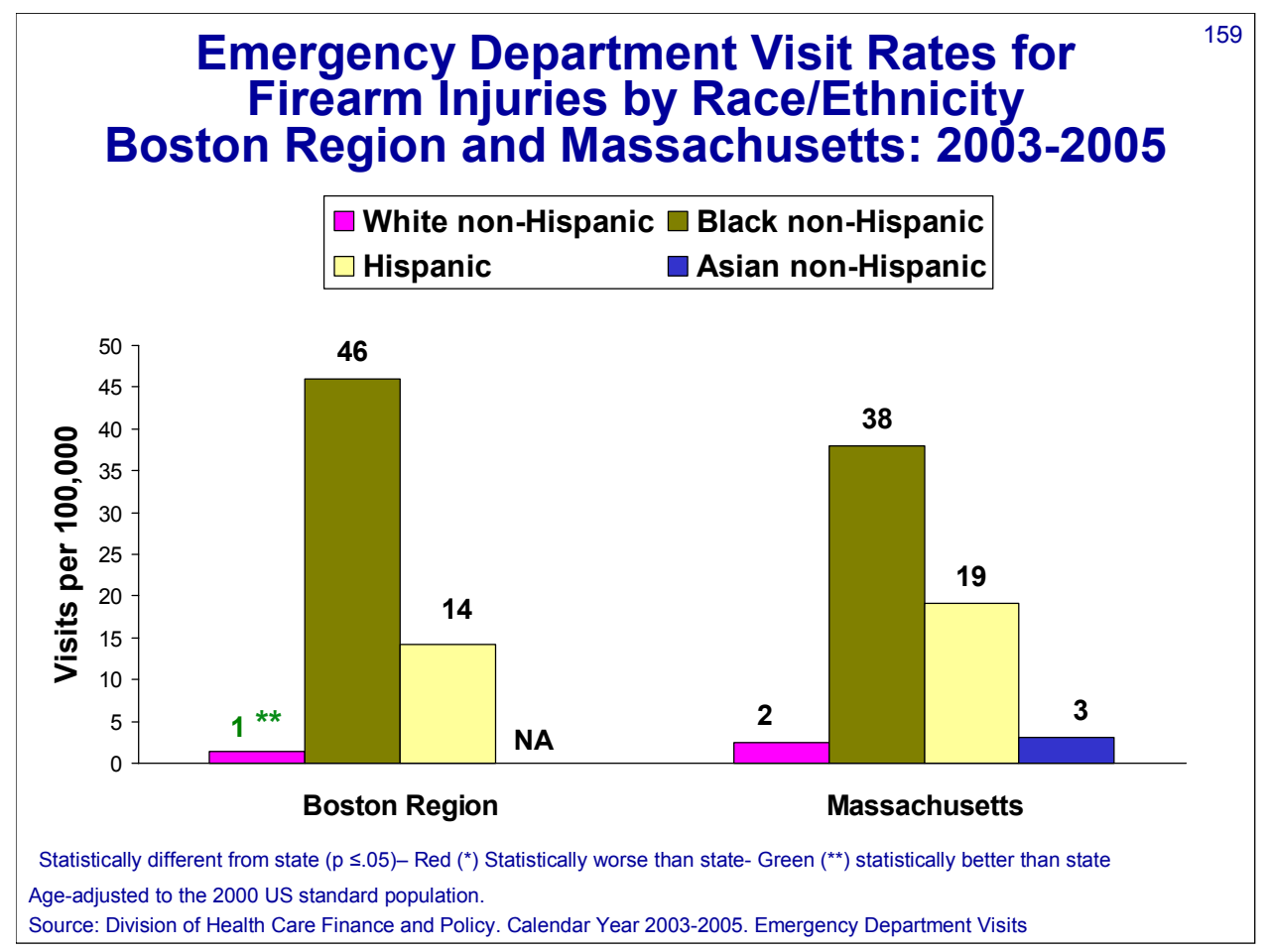


Firearm Death Rates by EOHHS Region Massachusetts: 2003-2005

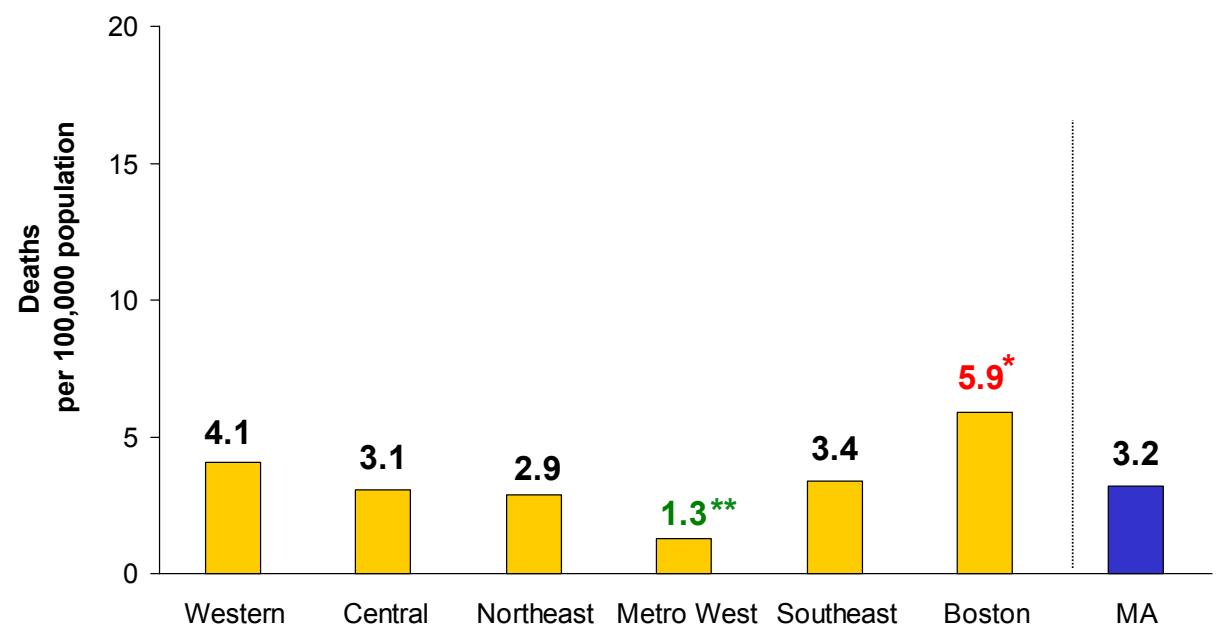

Statistically different from state $(p \leq .05)-\operatorname{Red}\left({ }^{*}\right)$ Statistically worse than state- Green $\left(^{* \star}\right)$ statistically better than state Age-adjusted to the 2000 US standard population.

\section{Firearm Death Rates}

Boston Region and Massachusetts: 2003-2005

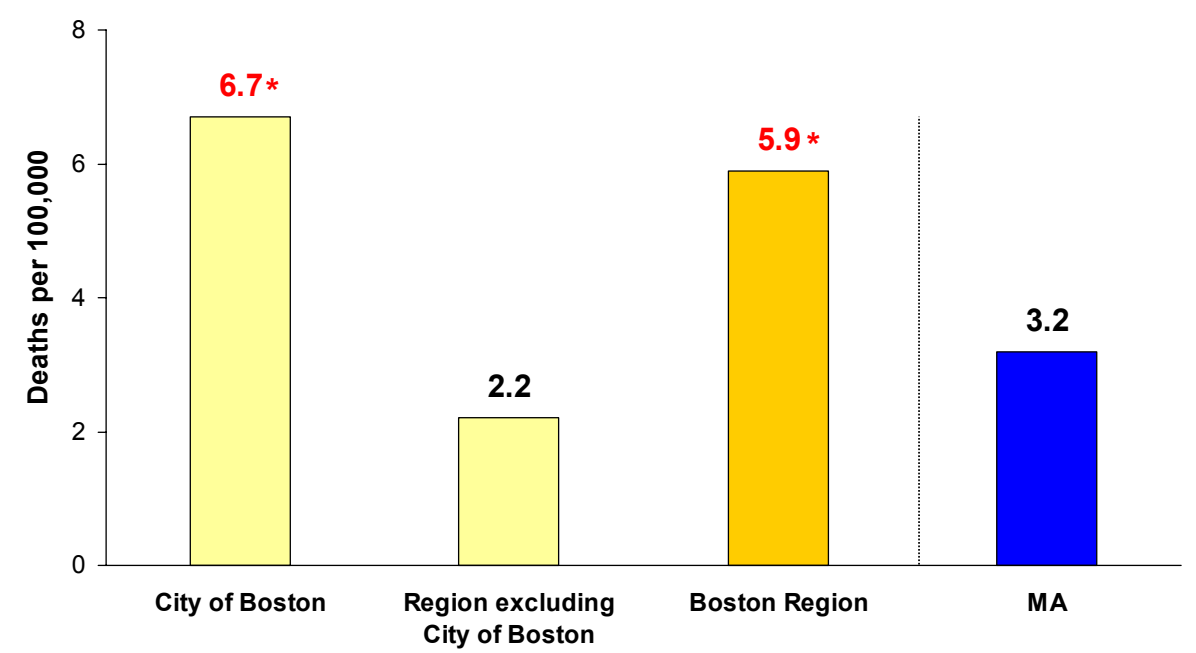

Statistically different from state $(p \leq .05)-\operatorname{Red}\left({ }^{*}\right)$ Statistically worse than state- Green $\left.{ }^{* *}\right)$ statistically better than state Age-adjusted to the 2000 US standard population

Source: MDPH, Health Information, Statistics, Research and Evaluation Bureau, Research \& Epidemiology Program 
Firearm Death Rates by Race/Ethnicity Boston Region and Massachusetts: 2003-2005

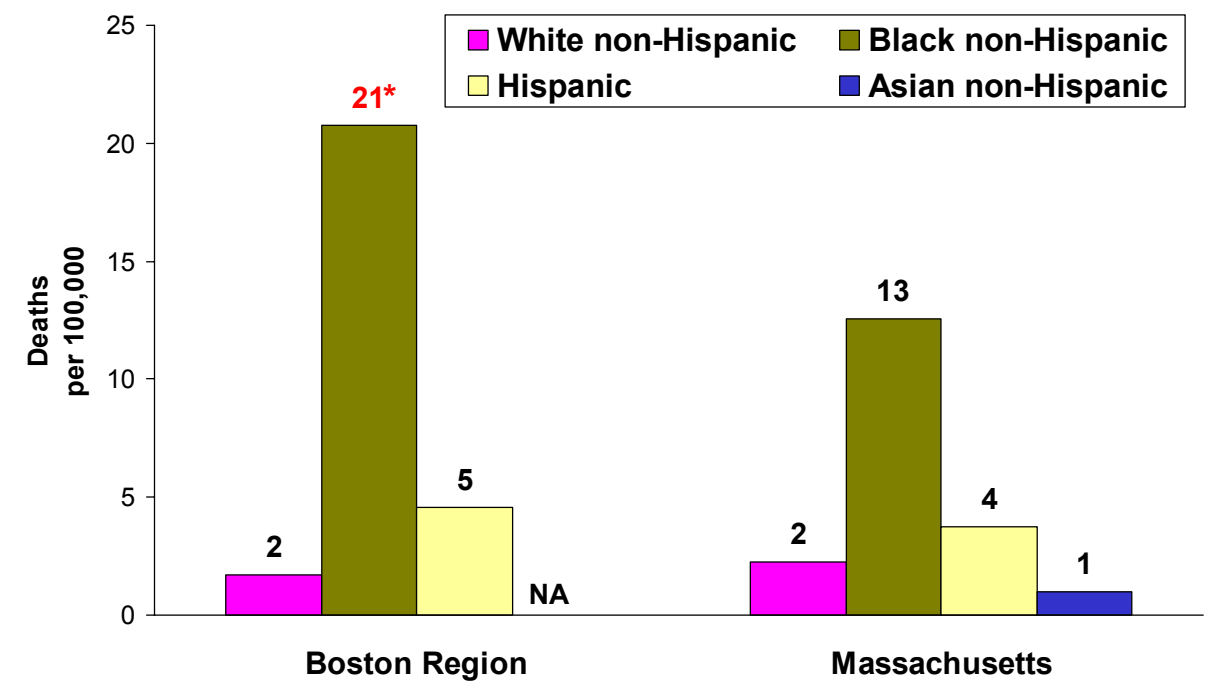

Statistically different from state $(p \leq .05)-\operatorname{Red}\left({ }^{*}\right)$ Statistically worse than state- Green $\left(^{* *}\right)$ statistically better than state Age-adjusted to the 2000 US standard population.

Source: MDPH, Health Information, Statistics, Research and Evaluation Bureau, Research \& Epidemiology Program

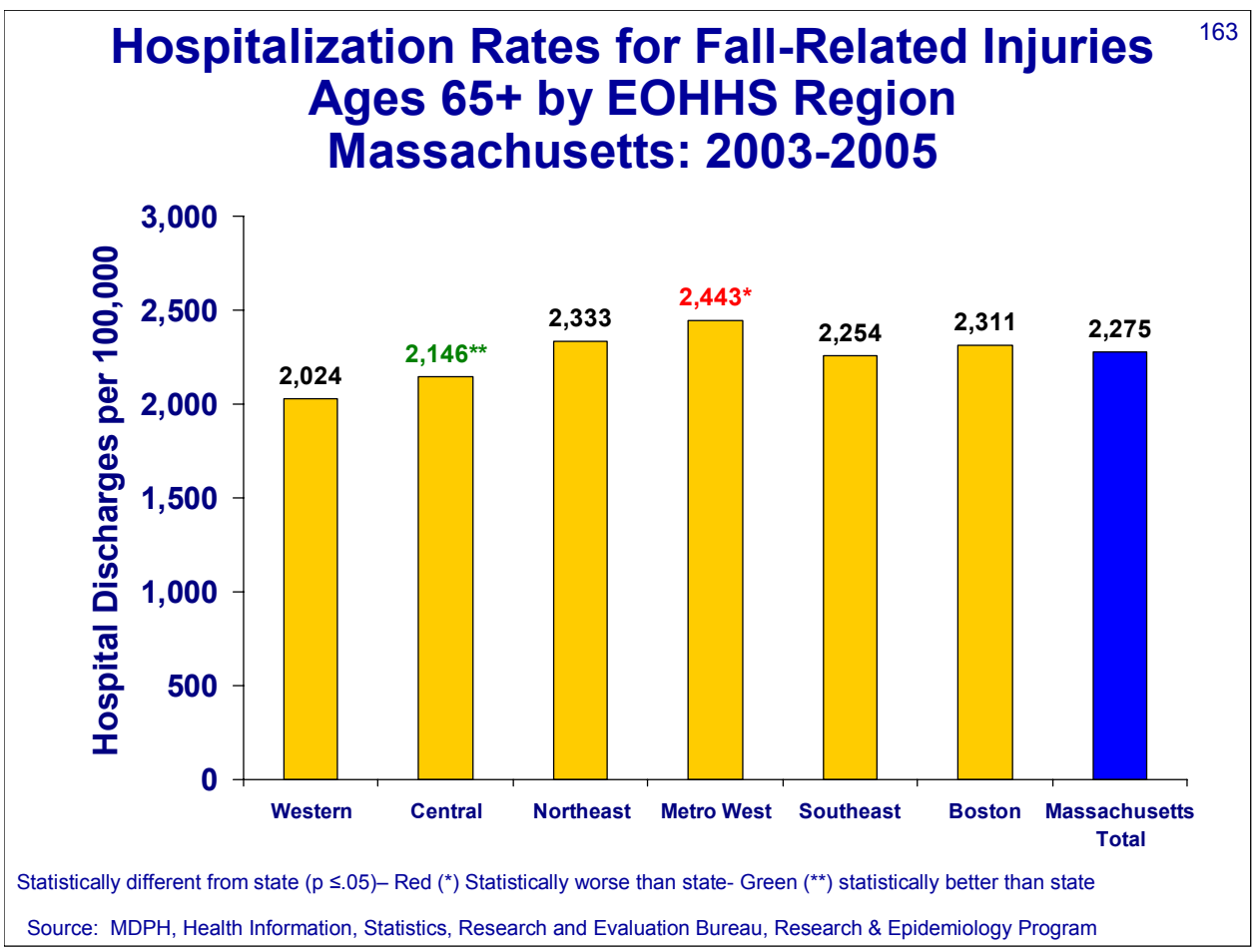




\section{Fall-related Injury Death Rates \\ Persons 65+ Years by EOHHS Region \\ Massachusetts: 2003-2005}

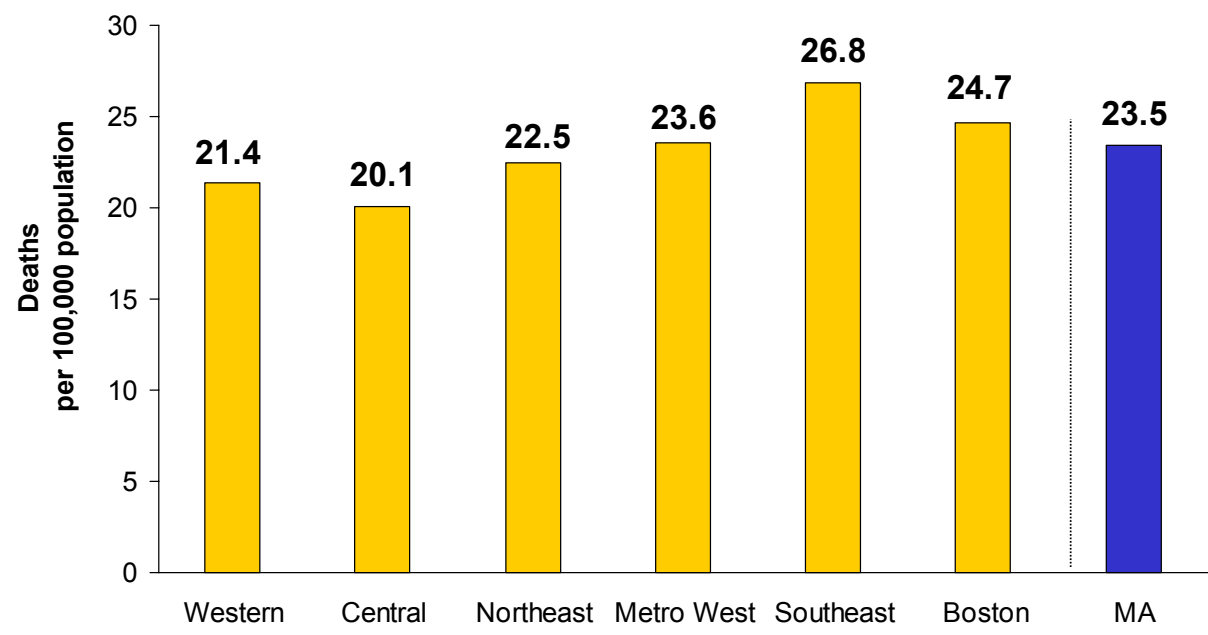

Statistically different from state $(p \leq .05)-\operatorname{Red}\left({ }^{*}\right)$ Statistically worse than state- Green $\left(^{* *}\right)$ statistically better than state

Hospital Discharge Rates for Fall-Related Injuries by EOHHS Region

Massachusetts: 2003-2005

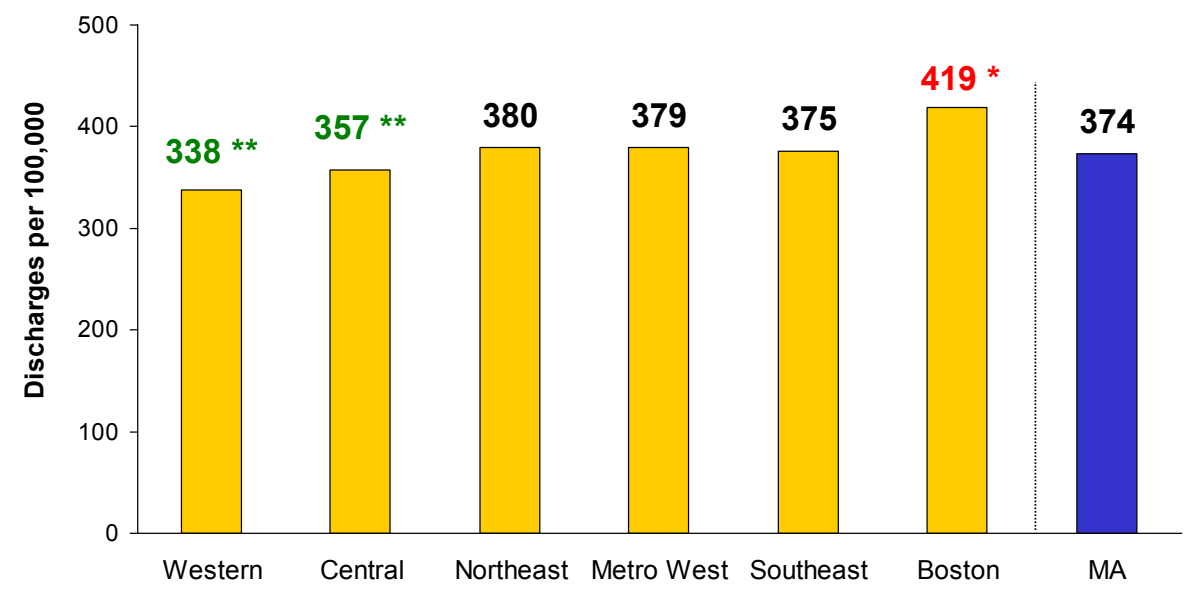

Statistically different from state $(p \leq .05)$ - Red $\left({ }^{*}\right)$ Statistically worse than state- Green $\left({ }^{* *}\right)$ statistically better than state Age-adjusted to the 2000 US standard population. 


\section{Hospital Discharge Rates for Fall-related Injuries by Race/Ethnicity, Boston Region and Massachusetts: 2003-2005}
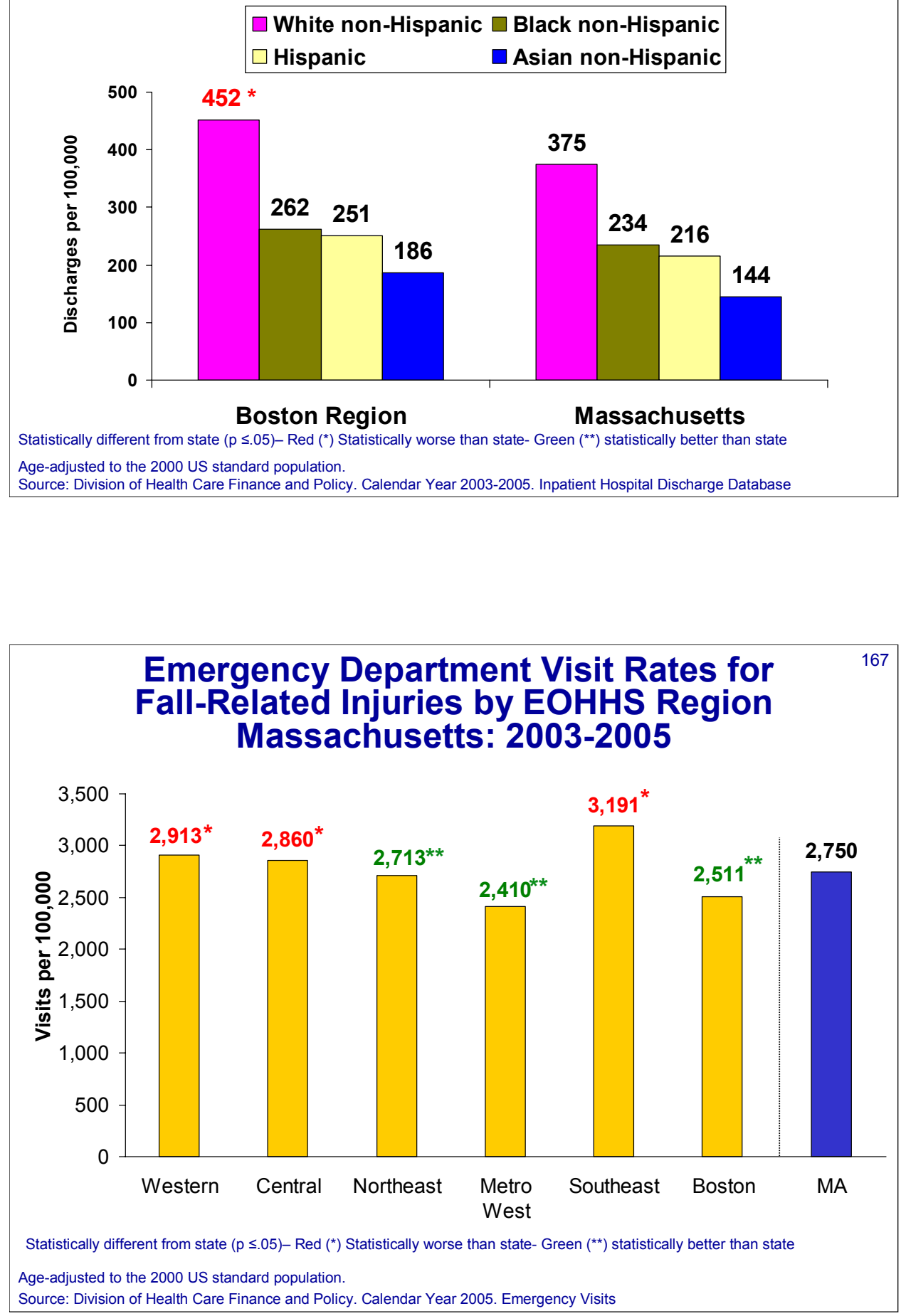

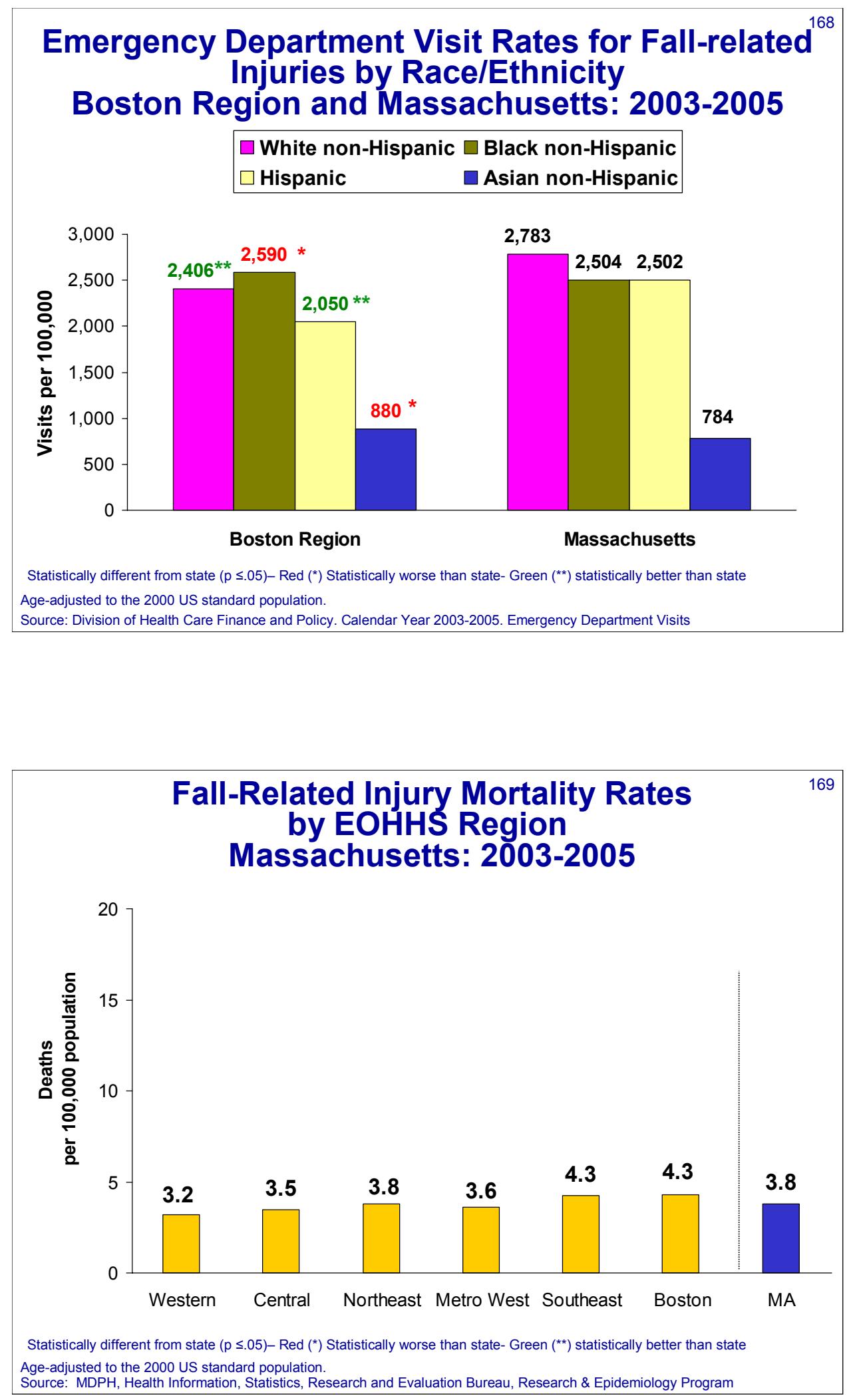
Fall-related Injury Death Rates by Race/Ethnicity Boston Region and Massachusetts: 2003-2005

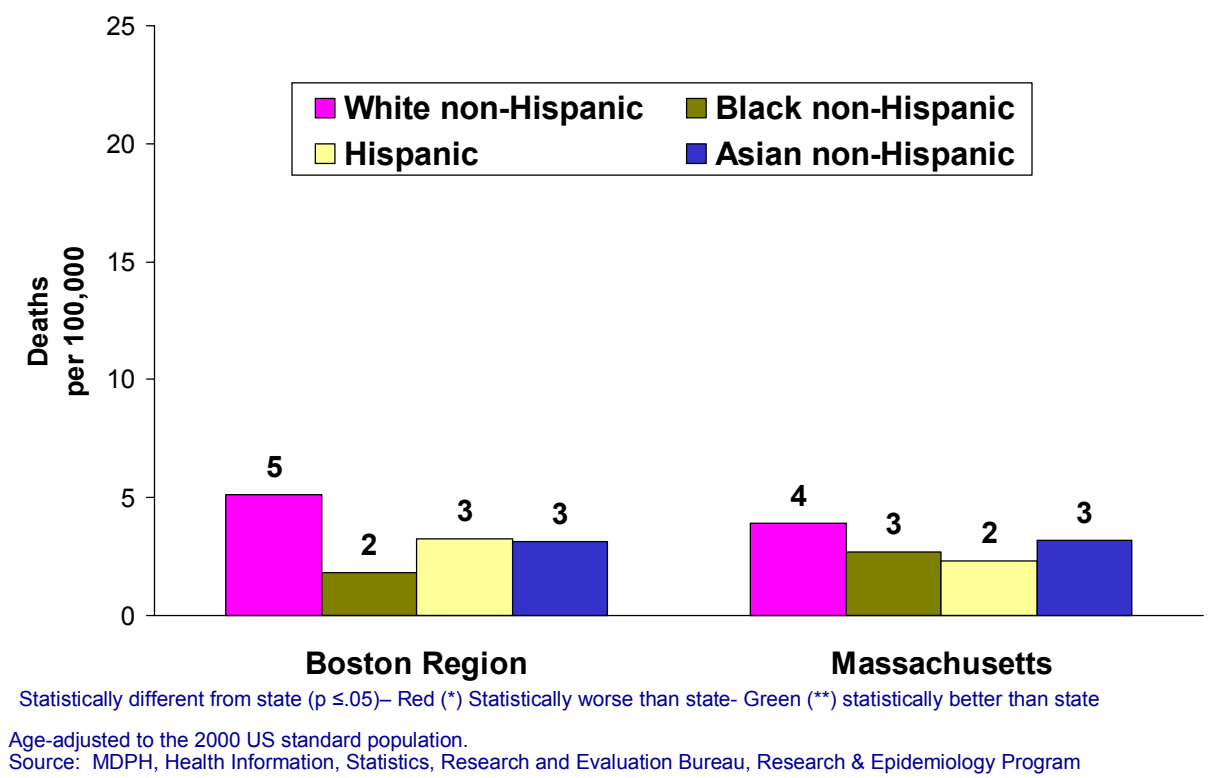

Age-adjusted to the 2000 US standard population

Source: MDPH, Health Information, Statistics, Research and Evaluation Bureau, Research \& Epidemiology Program

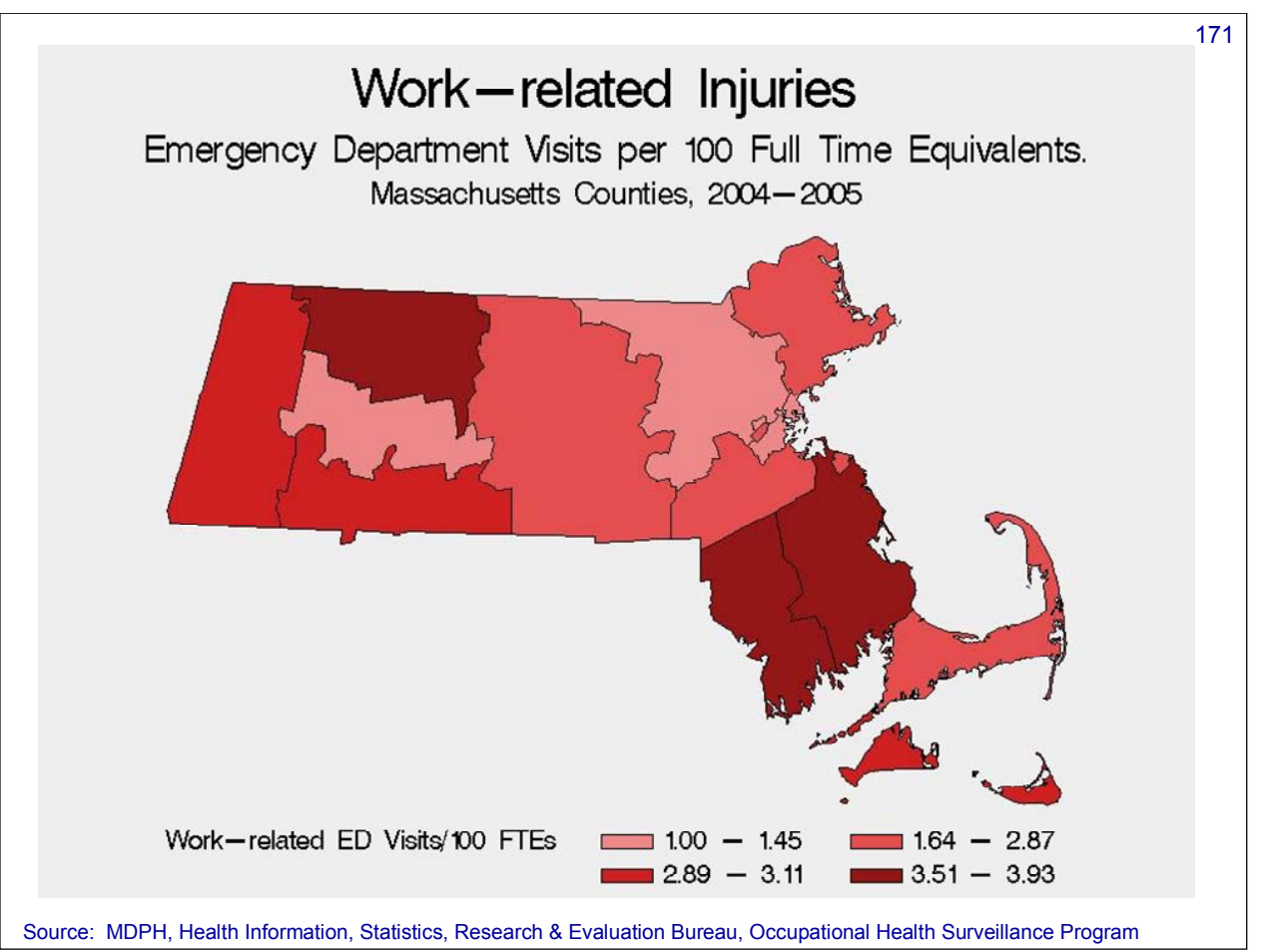




\section{Work-related Injuries among Teens}

Emergency Department Visits per 100 Full Time Equivalents Massachusetts Counties, 2004-2005

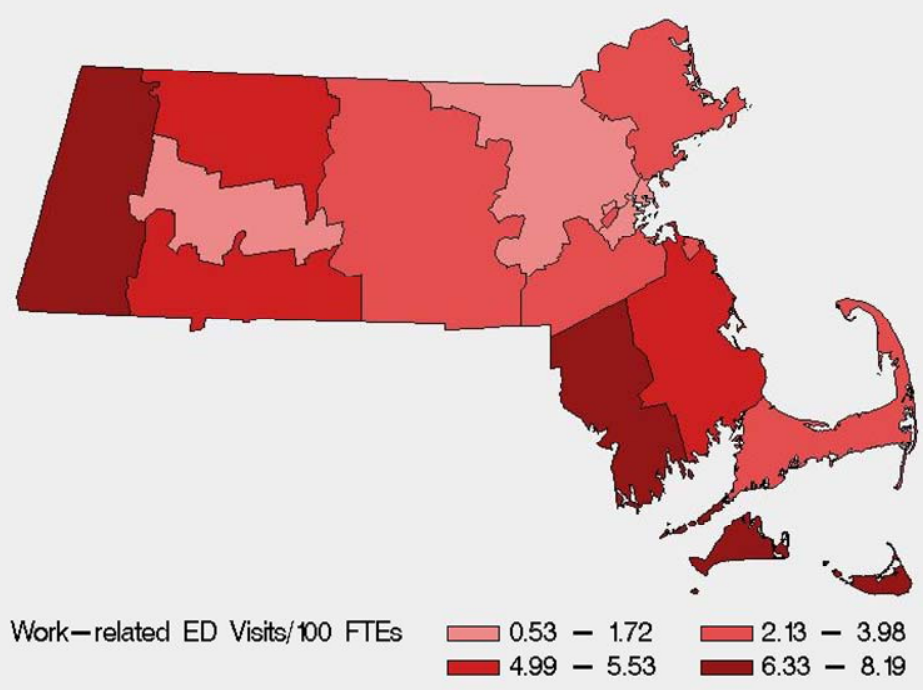

\section{Work- related Burn Injuries}

Emergency Department Visits per 10,000 Full Time Equivalents Massachusetts Counties, 2004-2005

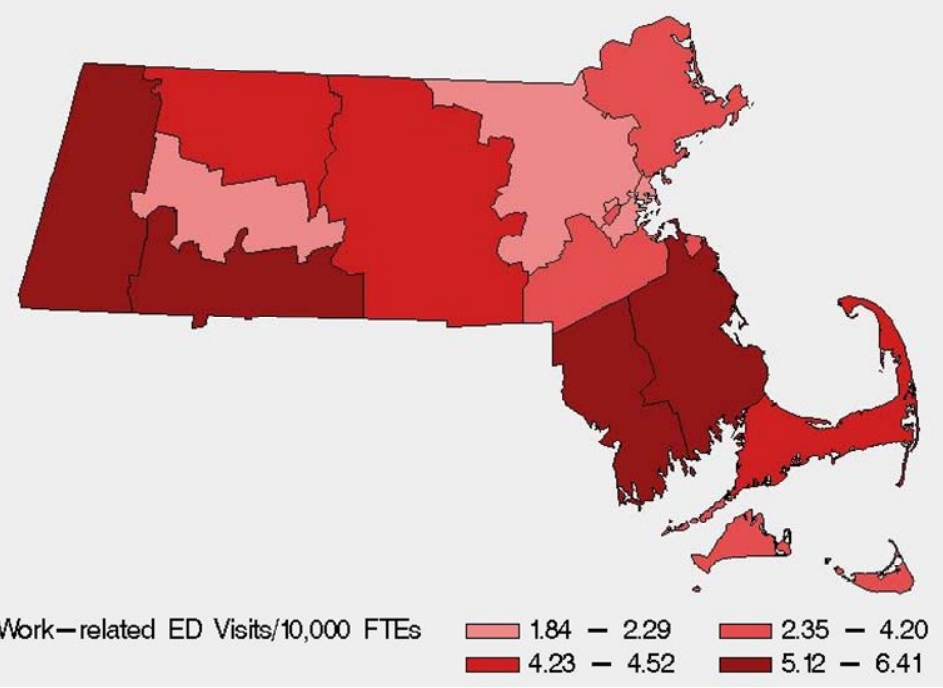

Source: MDPH, Health Information, Statistics, Research \& Evaluation Bureau, Occupational Health Surveillance Program 


\section{Work-related Amputations}

Emergency Department Visits per 10,000 Full Time Equivalents

Massachusetts Counties, 2004-2005

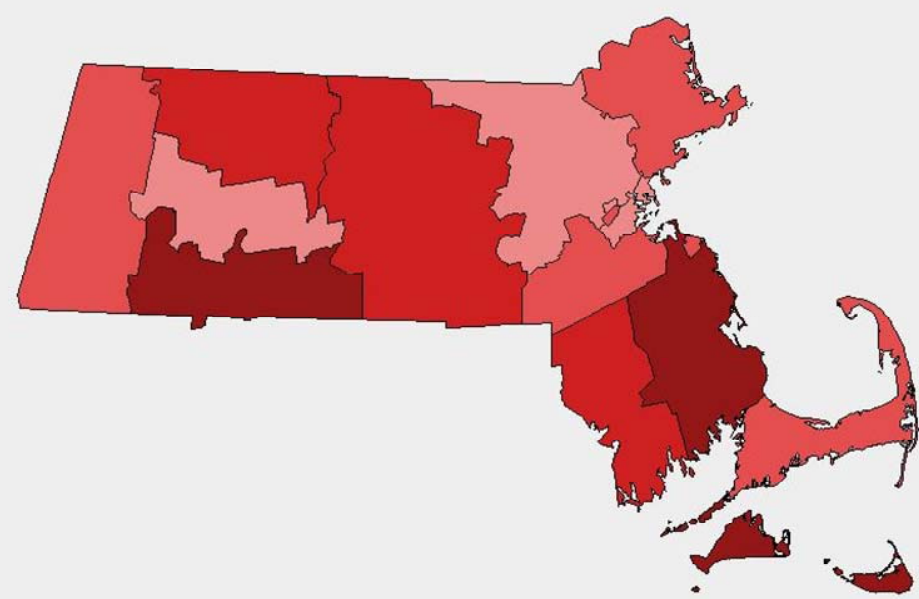

Work-related ED Visits/10,000 FTEs $\square 0.62-0.65 \square 0.69-1.07$

$$
\square 1.32-1.58 \square 1.67-2.70
$$

\section{Work-related Injuries}

Hospitalizations per 10,000 Full Time Equivalents.

Massachusetts Counties, 2004-2005

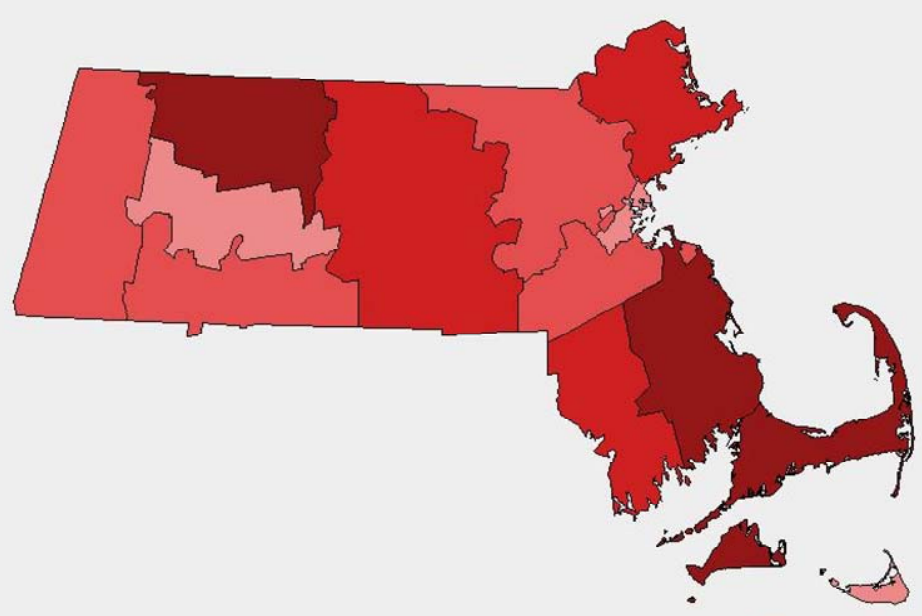

Work-related Hospitalizations/10,000 FTEs $\square 1.50-2.52 \square 2.69-4.37$

$$
\square .54-5.26 \square 5.49-6.76
$$

Source: MDPH, Health Information, Statistics, Research \& Evaluation Bureau, Occupational Health Surveillance Program 
Number and Rate of Fatal Occupational Injuries by year, Massachusetts: 1991- 2005

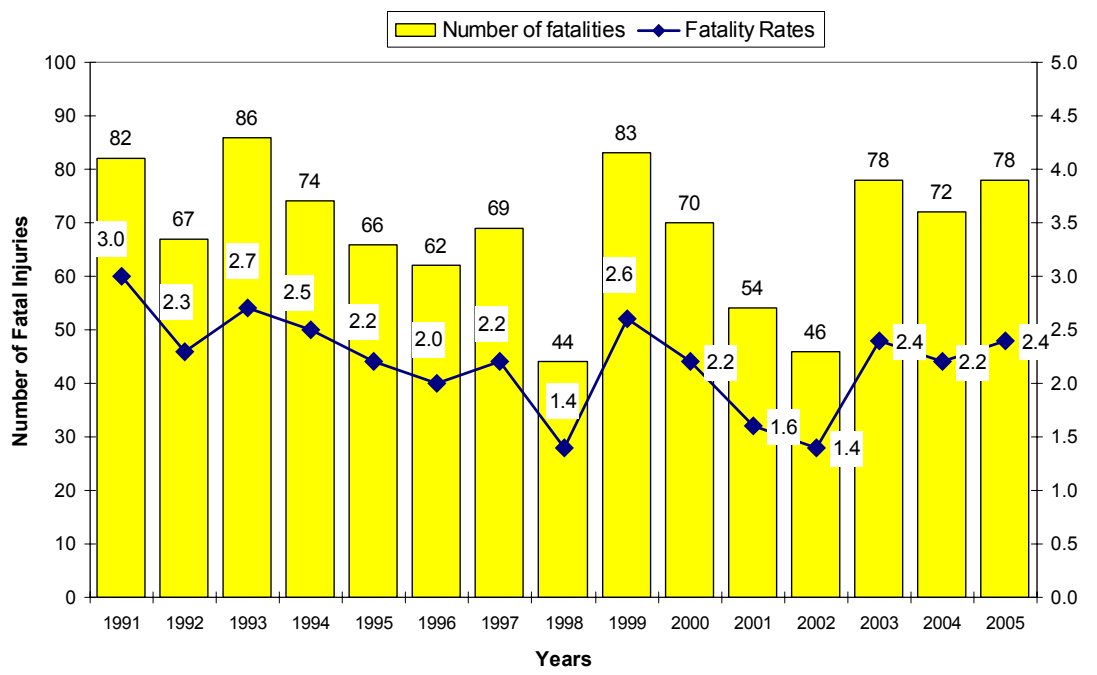

Source: MDPH, Health Information, Statistics, Research \& Evaluation Bureau, Occupational Health Surveillance ProgramCensus of Fatal Occupational Injuries (CFOI) 1991-2005. MA employment data from BLS Current Population Survey (1991 2005) was used to calculate rates.

\section{Summary Injury/Violence Hospitalization Indicators by EOHHS Region, Massachusetts: 2003-2005}

\begin{tabular}{|c|c|c|c|c|c|c|c|}
\hline $\begin{array}{c}\text { Hospital } \\
\text { Discharge } \\
\text { Indicator Rates } \\
\text { (age-adjusted) }\end{array}$ & $\begin{array}{c}\text { MA } \\
\text { Total }\end{array}$ & Western & Central & Northeast & $\begin{array}{l}\text { Metro } \\
\text { West }\end{array}$ & $\begin{array}{c}\text { South } \\
\text { East }\end{array}$ & Boston \\
\hline All Injuries & 836 & 872 & 830 & 836 & 753 & 859 & 966 \\
\hline $\begin{array}{l}\text { Fall-related } \\
\text { Injury, } \\
\text { Ages 65pls }\end{array}$ & 2,275 & 2,024 & 2,146 & 2,333 & 2,443 & 2,254 & 2,311 \\
\hline $\begin{array}{c}\text { Traumatic Brain } \\
\text { Injuries }\end{array}$ & 80 & 93 & 97 & 82 & 65 & 73 & 94 \\
\hline $\begin{array}{l}\text { Motor vehicle- } \\
\quad \text { related }\end{array}$ & 77 & 93 & 91 & 73 & 57 & 88 & 74 \\
\hline Self-Inflicted & 65 & 92 & 63 & 68 & 48 & 66 & 65 \\
\hline Assault & 28 & 34 & 24 & 23 & 14 & 21 & 72 \\
\hline $\begin{array}{l}\square \text { Better } \\
\text { e-adjusted to the } 2000 \\
\text { uurce: Division of Health }\end{array}$ & tcome (sig & ificantly differe & from state) & Worse Out & me (signific & $y$ different $f$ & state) \\
\hline
\end{tabular}




\section{Summary Injury/Violence Emergency Department Indicators by EOHHS Region, Massachusetts: 2003-2005}

\begin{tabular}{|c|c|c|c|c|c|c|c|}
\hline $\begin{array}{c}\text { Indicator } \\
\text { Age-adjusted Rate } \\
\text { per } 100,00\end{array}$ & $\begin{array}{l}\text { MA } \\
\text { Total }\end{array}$ & Western & Central & Northeast & $\begin{array}{l}\text { Metro } \\
\text { West }\end{array}$ & $\begin{array}{l}\text { South } \\
\text { East }\end{array}$ & Boston \\
\hline $\begin{array}{l}\text { ER Visit Rate } \\
\text { for all Injuries }\end{array}$ & 11,298 & 12,323 & 11,379 & 11,195 & 9,112 & 13,996 & 10,539 \\
\hline $\begin{array}{l}\text { ER Visit Rate } \\
\text { for Traumatic } \\
\text { Brain Injury }\end{array}$ & 604 & 461 & 646 & 662 & 563 & 653 & 640 \\
\hline $\begin{array}{l}\text { ER Visit Rate } \\
\text { for Firearm }\end{array}$ & 7 & 13 & 5 & 6 & 2 & 8 & 13 \\
\hline $\begin{array}{l}\text { ER Visit Rate } \\
\text { for Fall-related } \\
\text { injuries }\end{array}$ & 2,750 & 2,913 & 2,860 & 2,713 & 2,410 & 3,191 & 2,511 \\
\hline
\end{tabular}

\section{Summary Injury/Violence Mortality Indicators by EOHHS Region, Massachusetts: 2003-2005}

\begin{tabular}{|c|c|c|c|c|c|c|c|}
\hline $\begin{array}{c}\text { Mortality } \\
\text { Indicator } \\
\text { Age-adjusted Rate } \\
\text { per } 100,000\end{array}$ & $\begin{array}{c}\text { MA } \\
\text { Total }\end{array}$ & Western & Central & Northeast & $\begin{array}{l}\text { Metro } \\
\text { West }\end{array}$ & $\begin{array}{l}\text { South } \\
\text { East }\end{array}$ & Boston \\
\hline $\begin{array}{l}\text { Homicide } \\
\text { Death Rate }\end{array}$ & 2.6 & 3.3 & 1.7 & 1.8 & 0.7 & 2.6 & 7.3 \\
\hline $\begin{array}{c}\text { Suicide Death } \\
\text { Rate }\end{array}$ & 6.6 & 7.9 & 7.2 & 7.4 & 5.3 & 6.9 & 5.5 \\
\hline $\begin{array}{c}\text { Motor Vehicle- } \\
\text { related Death } \\
\text { Rate }\end{array}$ & 7.7 & 10.2 & 9.8 & 6.4 & 5.4 & 10.7 & 4.6 \\
\hline $\begin{array}{c}\text { Firearm Death } \\
\text { Rate }\end{array}$ & 3.2 & 4.1 & 3.1 & 2.9 & 1.3 & 3.4 & 5.9 \\
\hline $\begin{array}{c}\text { Fall-related } \\
\text { Injury Death } \\
\text { Rate }\end{array}$ & 3.8 & 3.2 & 3.5 & 3.8 & 3.6 & 4.3 & 4.3 \\
\hline \multicolumn{4}{|c|}{ Better Outcome (significantly different from state) } & \multicolumn{4}{|c|}{ Worse Outcome (significantly different from state) } \\
\hline
\end{tabular}




\section{HIVIAIDS}




\section{Persons Living with HIV by EOHHS Region Massachusetts: 2005}
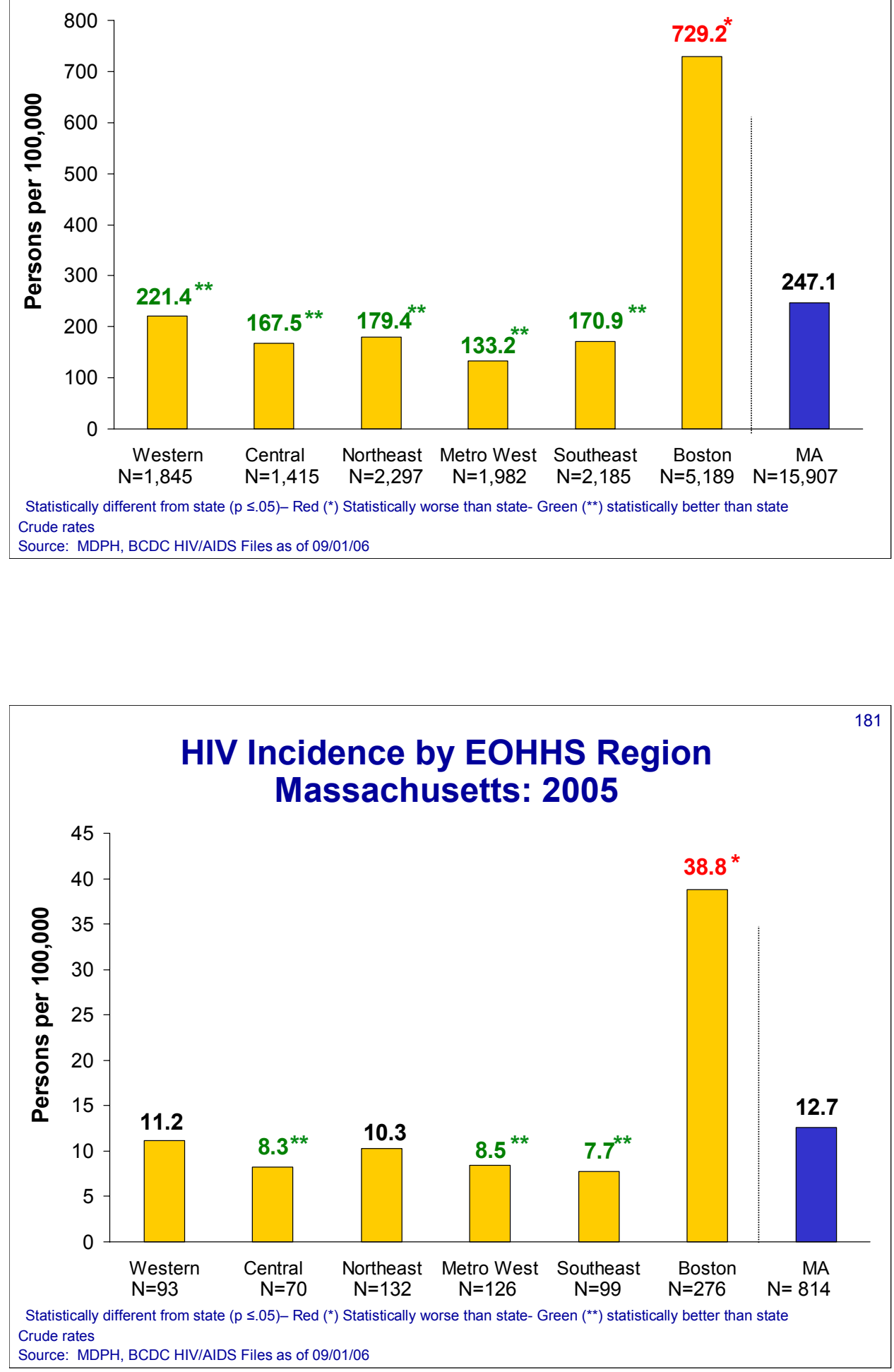


\section{Cities in Boston Region \& Massachusetts : 2005}

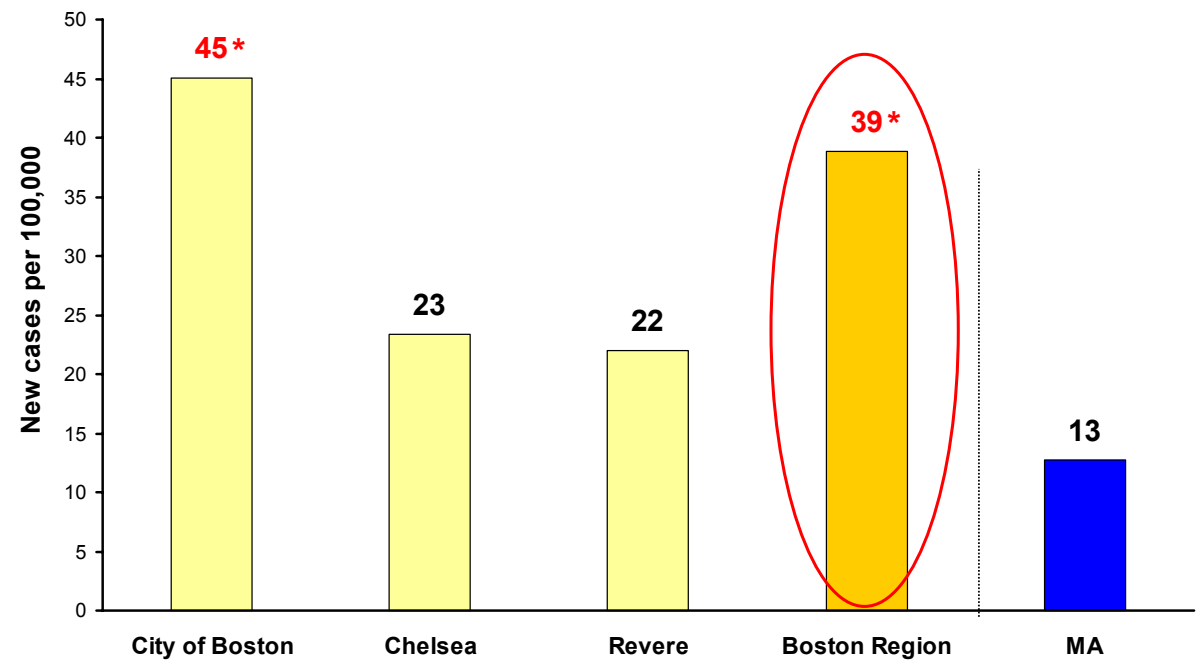

Statistically different from state $(p \leq .05)-\operatorname{Red}\left({ }^{*}\right)$ Statistically worse than state- Green $\left(^{\star *}\right)$ statistically better than state Crude rates

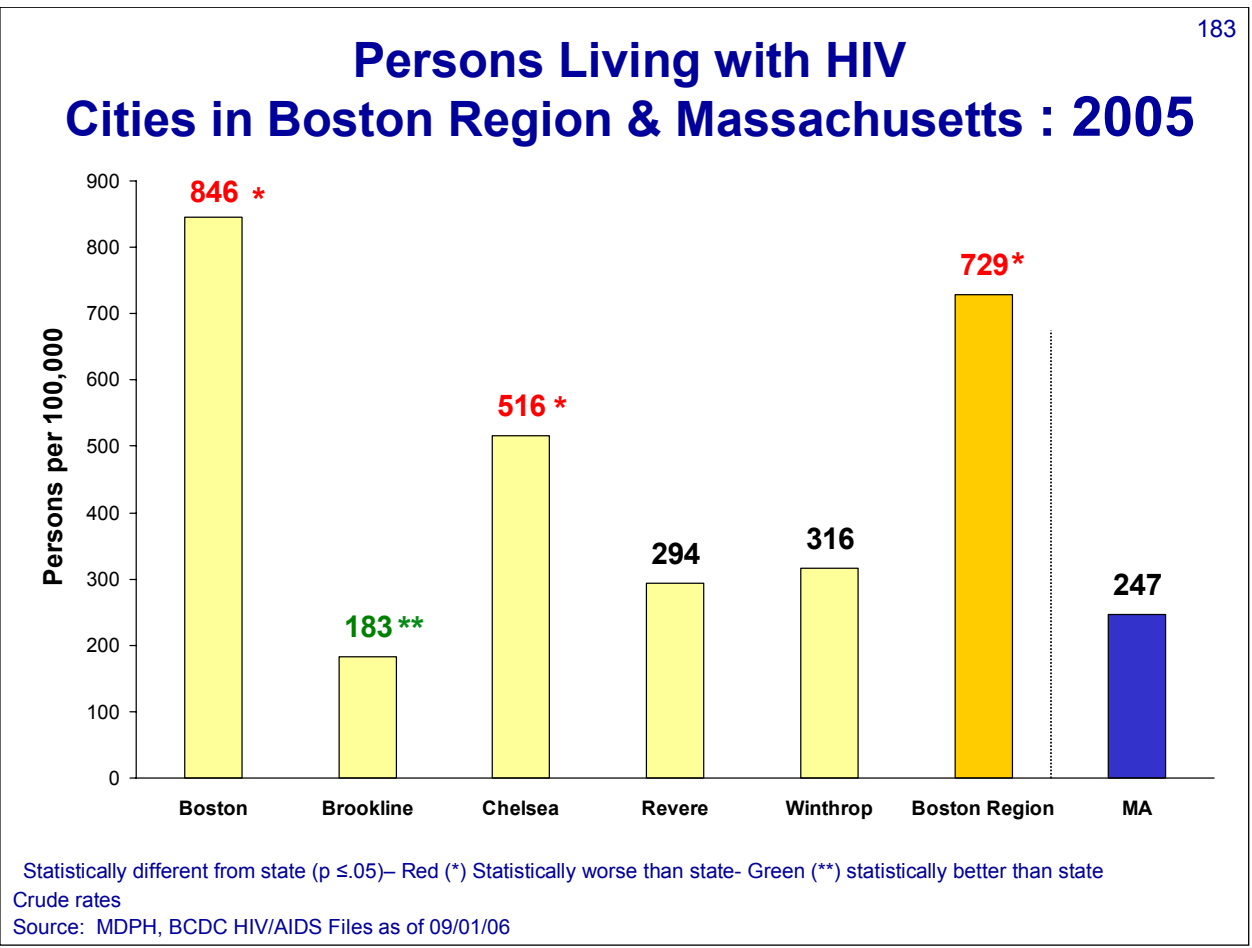



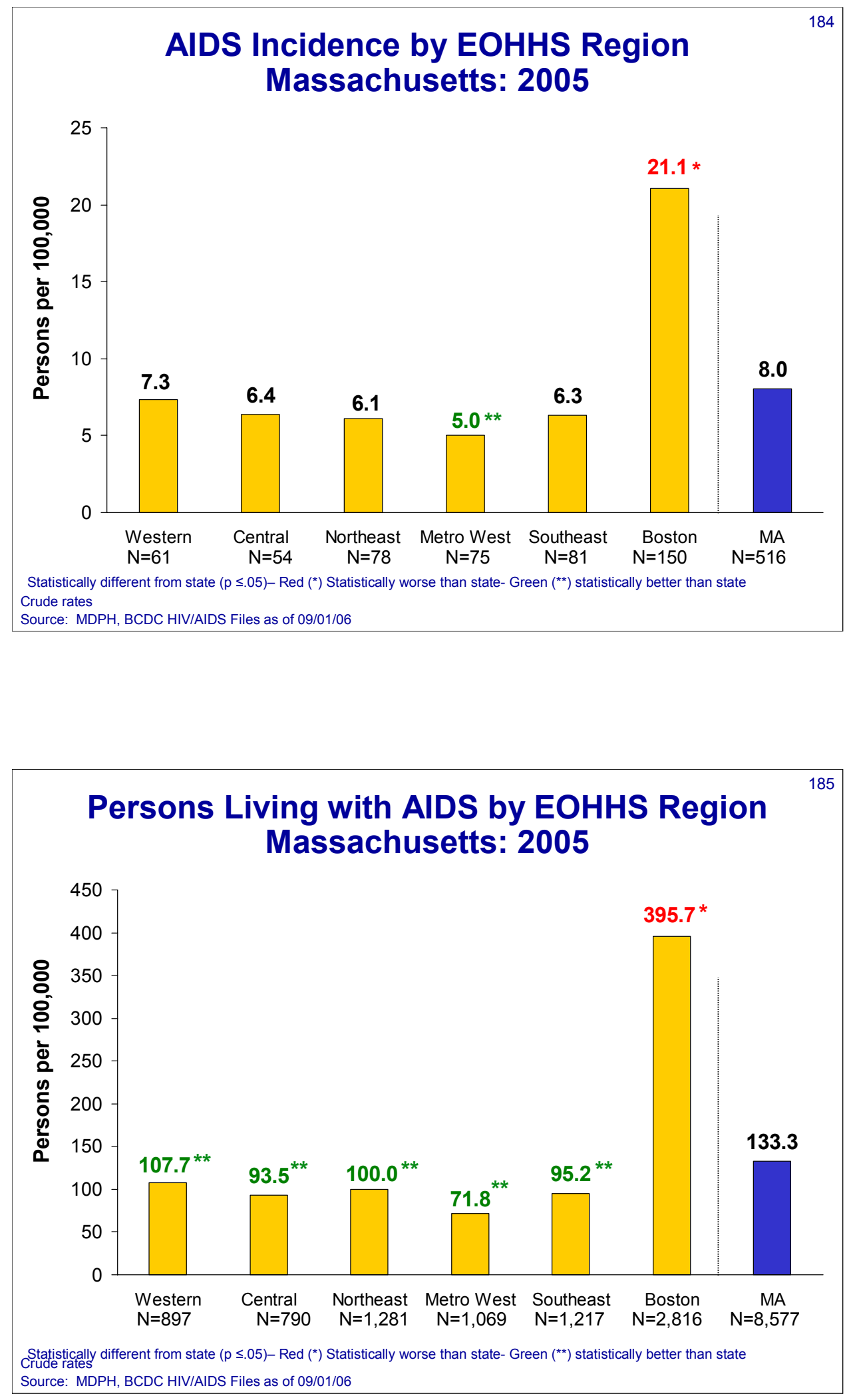
HIVIAIDS Mortality Rates by EOHHS Region, Massachusetts: 2003-2005

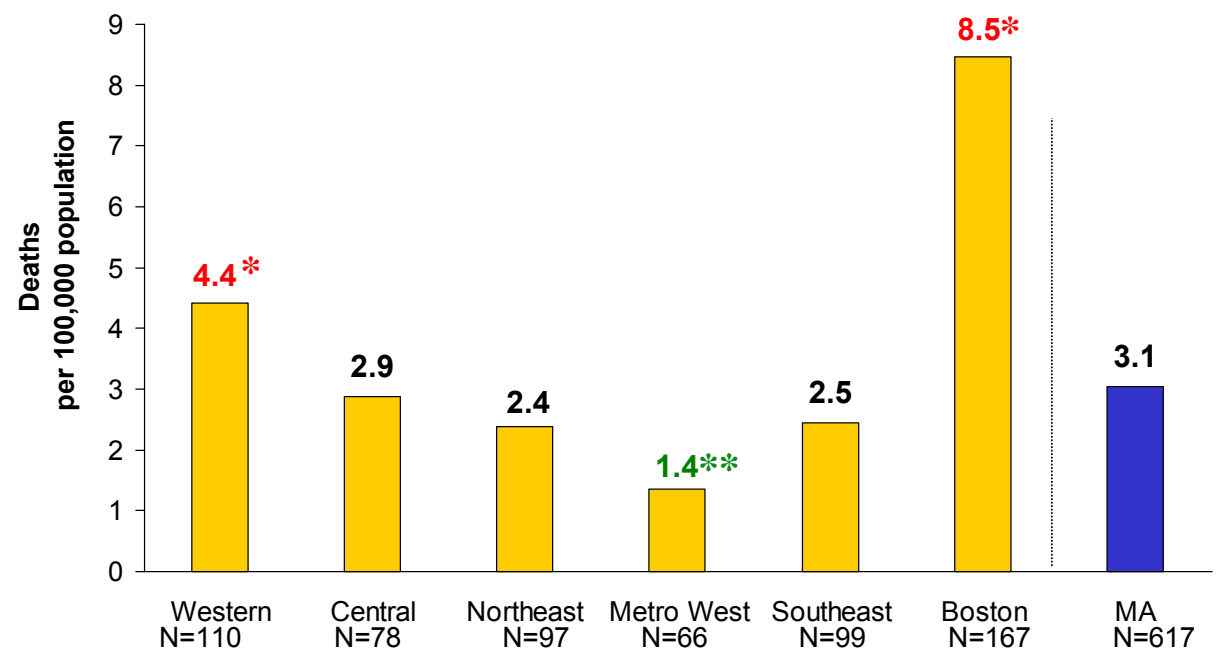

Statistically different from state $(p \leq .05)-\operatorname{Red}\left({ }^{*}\right)$ Statistically worse than state- Green $\left(^{* \star}\right)$ statistically better than state

HIVIAIDS Mortality Rates in

Boston Region and Massachusetts: 2003-2005

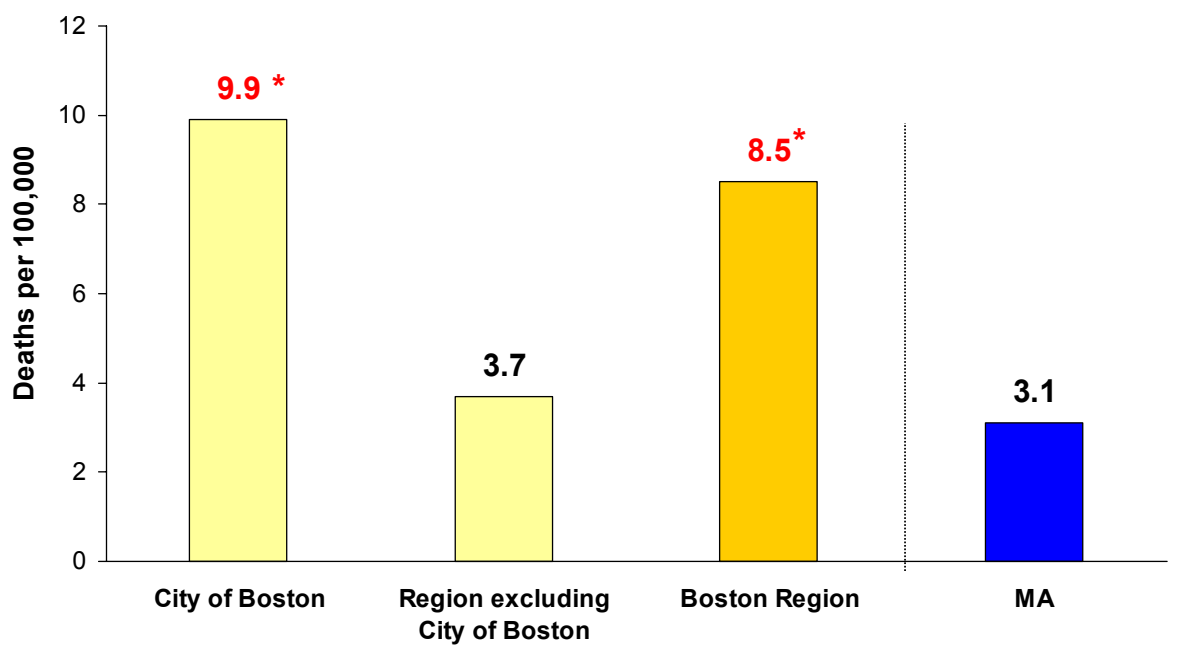

Statistically different from state $(p \leq .05)-\operatorname{Red}\left({ }^{*}\right)$ Statistically worse than state- Green $\left.{ }^{* *}\right)$ statistically better than state Age-adjusted to the 2000 US standard population

Source: MDPH, Health Information, Statistics, Research and Evaluation Bureau, Research \& Epidemiology Program 


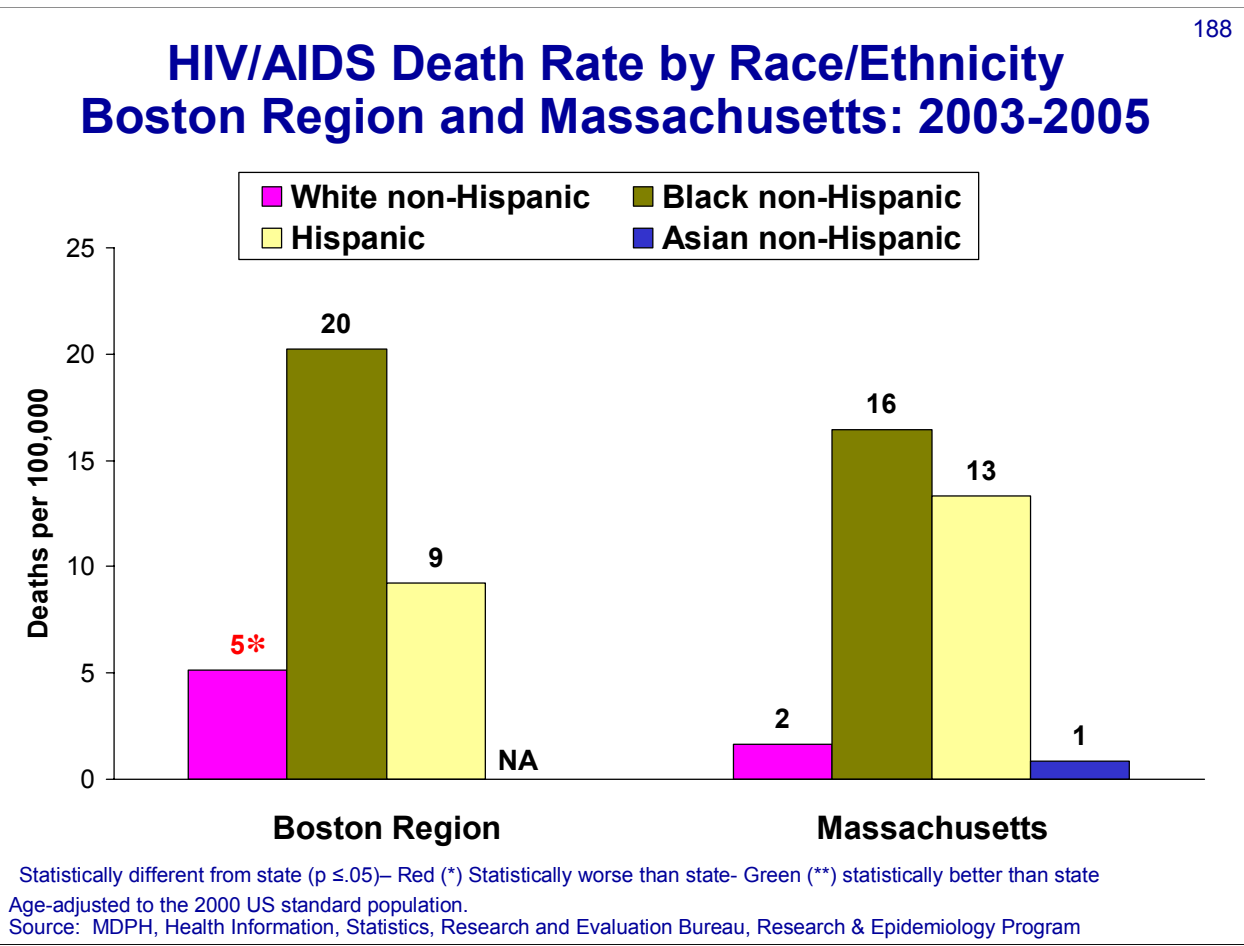

\begin{tabular}{|c|c|c|c|c|c|c|c|}
\hline \multicolumn{8}{|c|}{$\begin{array}{c}\text { Summary HIVIAIDS Indicators } \\
\text { by EOHHS Region }\end{array}$} \\
\hline $\begin{array}{l}\text { Indicator } \\
\text { Crude rate, per } \\
100,000\end{array}$ & $\begin{array}{l}\text { MA } \\
\text { Total }\end{array}$ & Western & Central & Northeast & $\begin{array}{l}\text { Metro } \\
\text { West }\end{array}$ & $\begin{array}{c}\text { South } \\
\text { East }\end{array}$ & Boston \\
\hline $\begin{array}{l}\text { Persons living with } \\
\text { HIV (2005) }\end{array}$ & 247.1 & 221.4 & 167.5 & 179.4 & 133.1 & 170.9 & 729.2 \\
\hline $\begin{array}{c}\text { HIV } \\
\text { Incidence(2005) }\end{array}$ & 12.6 & 11.2 & 8.3 & 10.3 & 8.5 & 7.7 & 38.8 \\
\hline $\begin{array}{l}\text { Persons living with } \\
\text { AIDS (2005) }\end{array}$ & 133.2 & 107.7 & 93.5 & 100.0 & 71.8 & 95.2 & 395.7 \\
\hline $\begin{array}{l}\text { AIDS Incidence } \\
(2005)\end{array}$ & 8.0 & 7.3 & 6.4 & 6.1 & 5.0 & 6.3 & 21.1 \\
\hline $\begin{array}{l}\text { HIVIAIDS Mortality } \\
(2003-2005)\end{array}$ & 3.1 & 4.4 & 2.9 & 2.4 & 1.4 & 2.5 & 8.5 \\
\hline
\end{tabular}




\section{INFECTIOUS DISEASES}




\section{Chlamydia Incidence Boston Region and Massachusetts: 1989-2005}
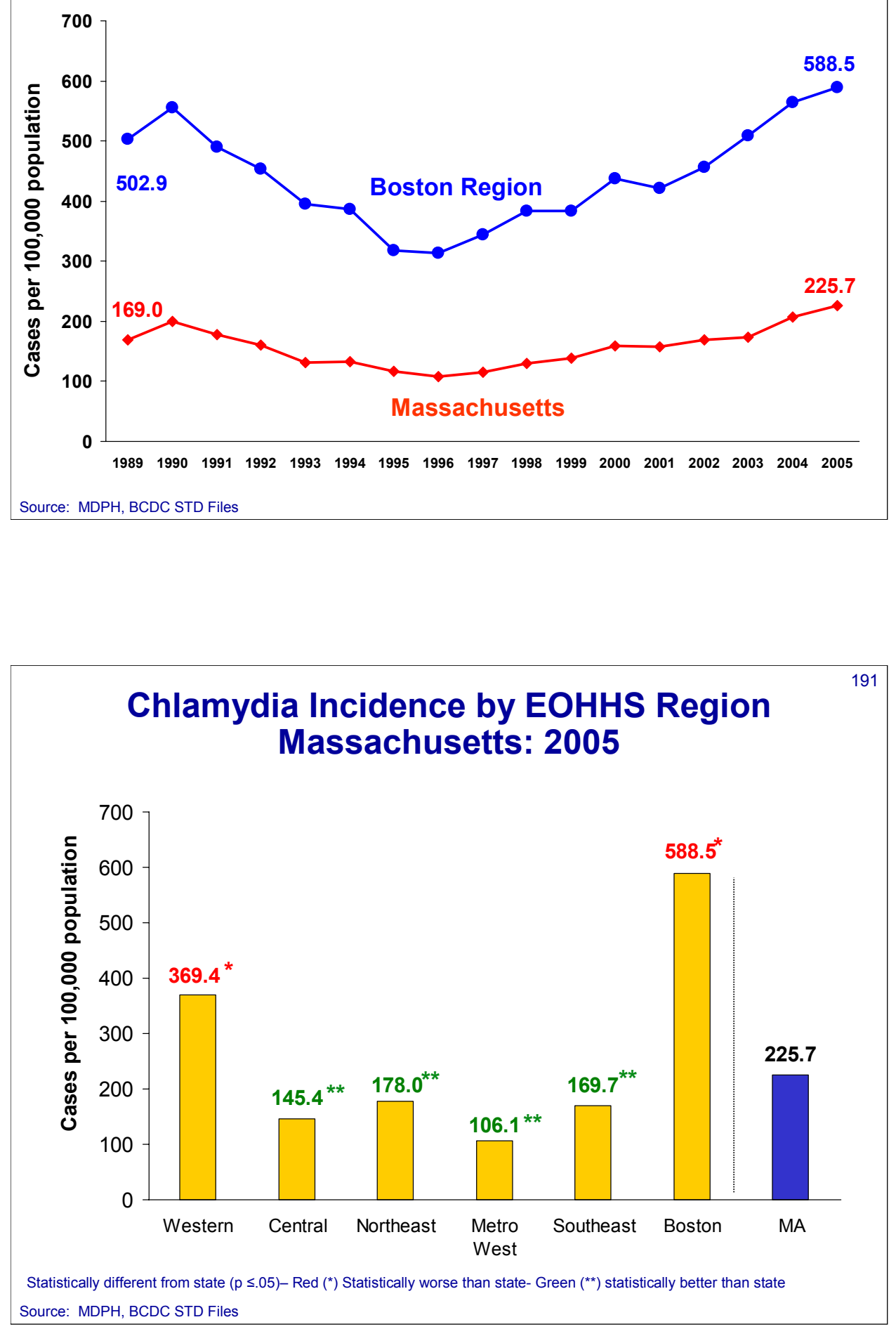
Chlamydia Incidence Rate by Emergency

Preparation Region, 2006

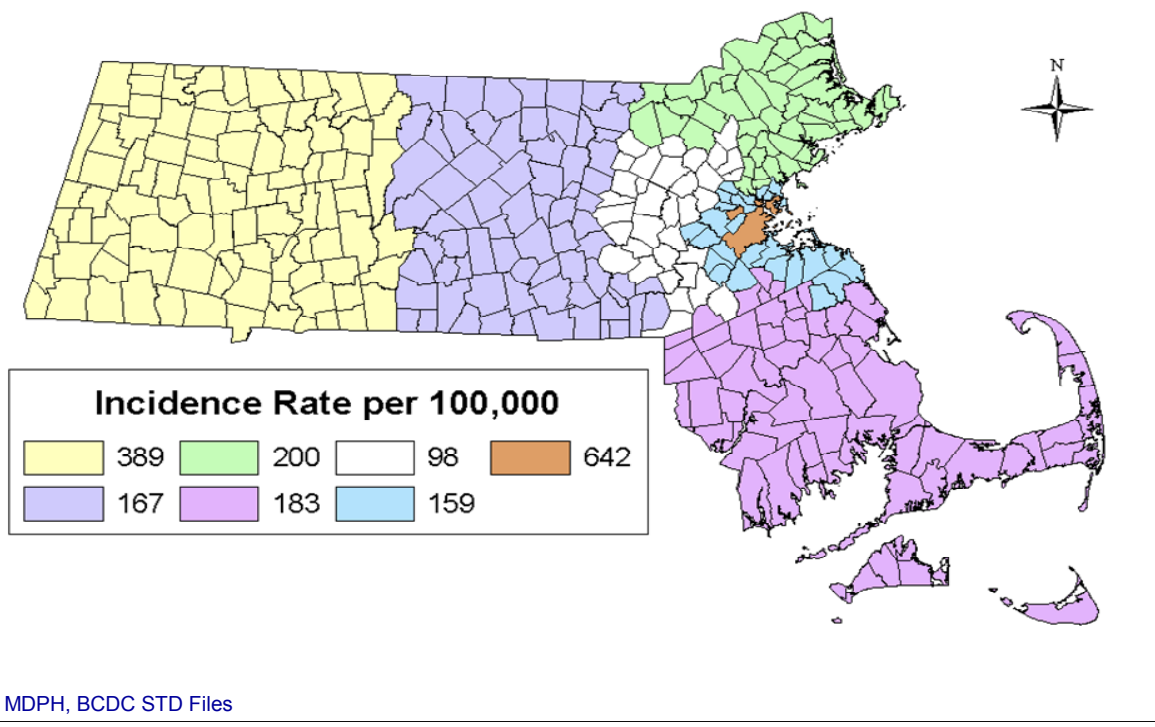

Source: MDPH, BCDC STD Files

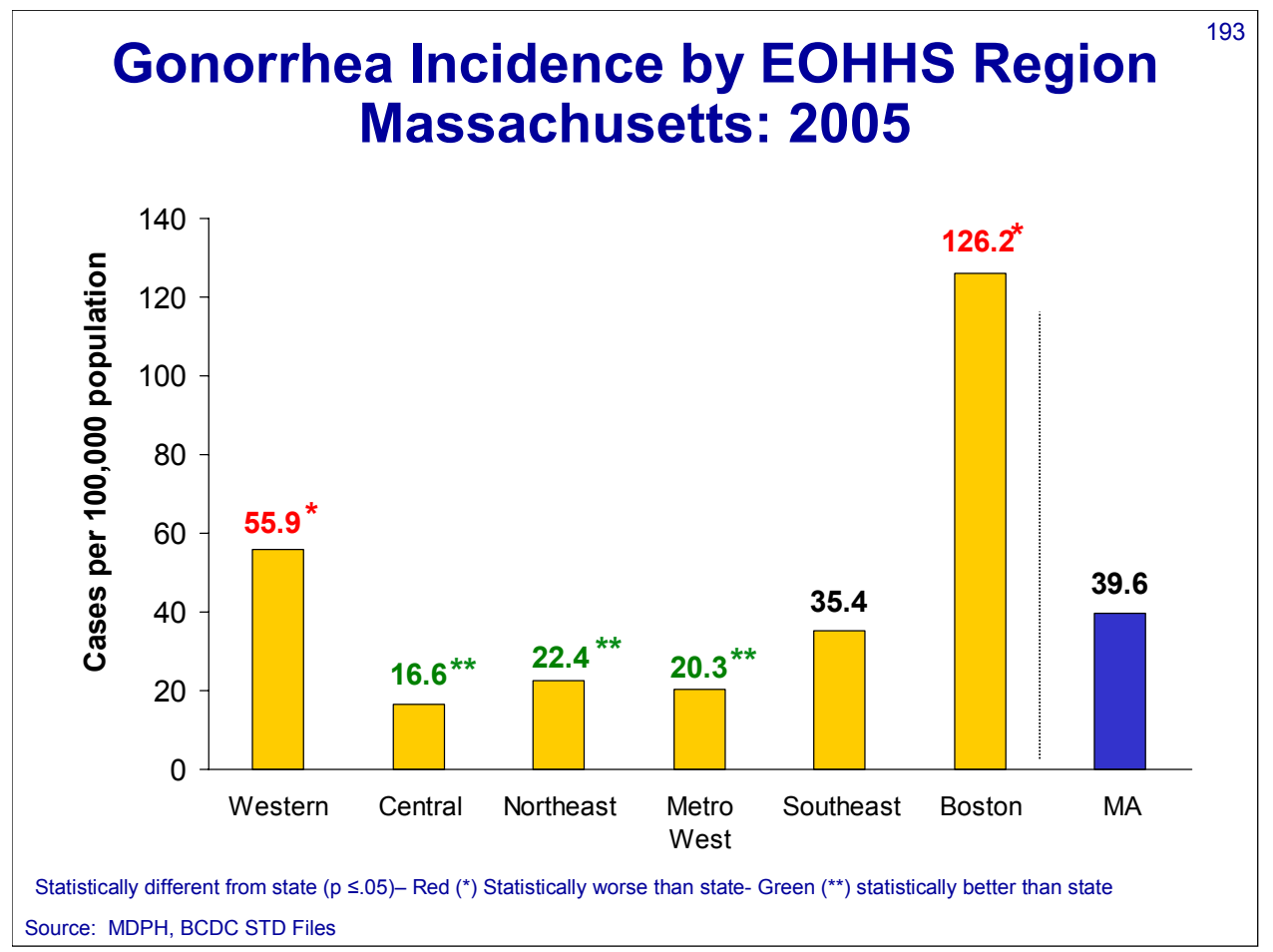



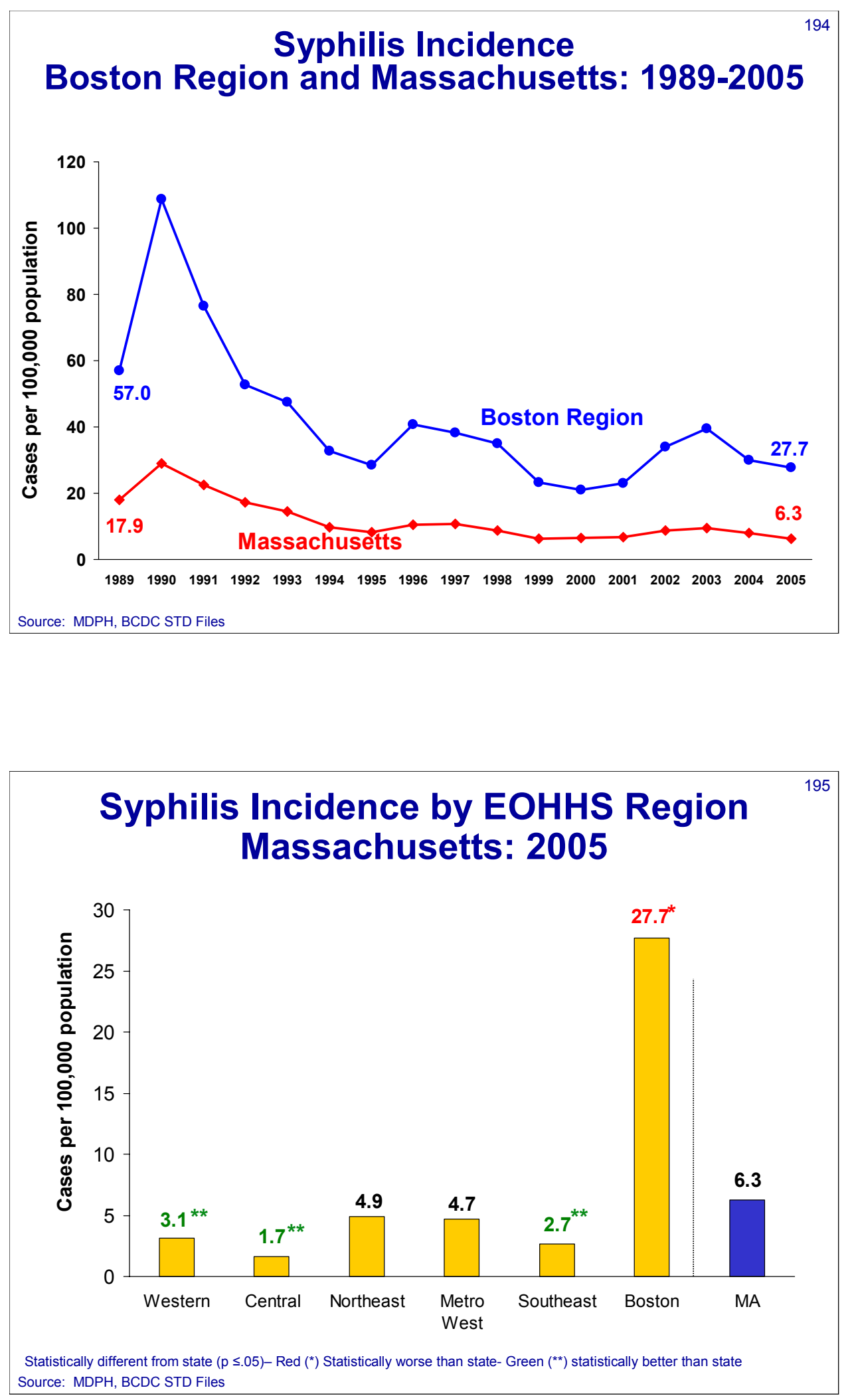
Tuberculosis Incidence by EOHHS Region Massachusetts: 2004

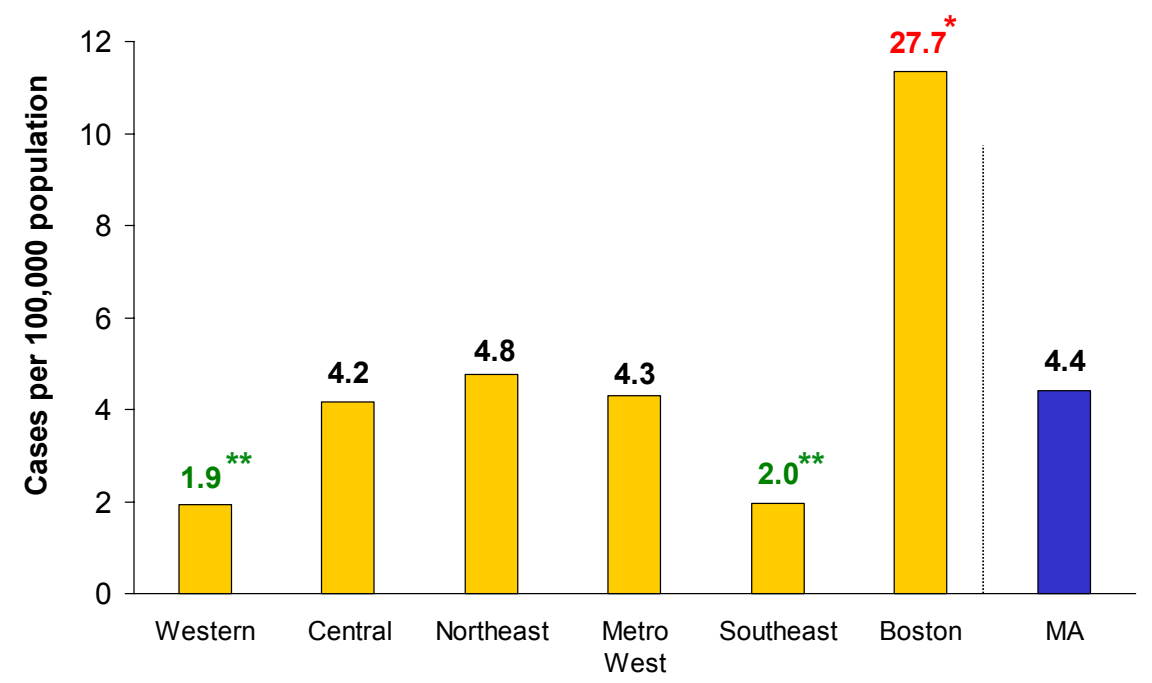

Statistically different from state $(p \leq .05)-\operatorname{Red}\left({ }^{*}\right)$ Statistically worse than state- Green $\left(^{* \star}\right)$ statistically better than state Source: MDPH Bureau of Communicable Disease Control, ISIS

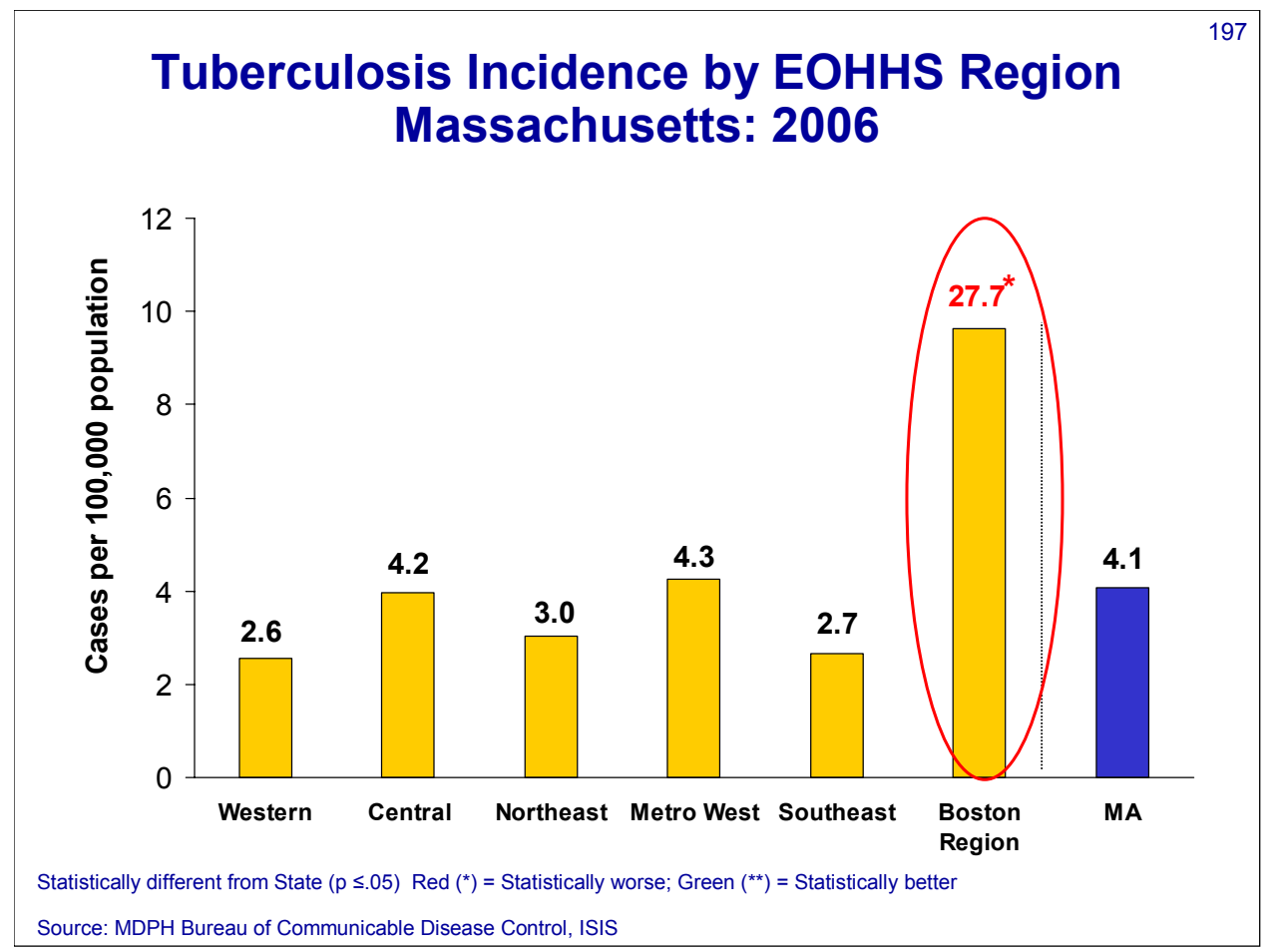


Confirmed Tuberculosis Cases by Race/Ethnicity and EOHHS Region Massachusetts: 2006

\begin{tabular}{|l|c|c|c|c|}
\hline \multicolumn{1}{|c|}{$\begin{array}{c}\text { EOHHS } \\
\text { Region }\end{array}$} & $\begin{array}{c}\text { White non- } \\
\text { Hispanic }\end{array}$ & $\begin{array}{c}\text { Black non- } \\
\text { Hispanic }\end{array}$ & $\begin{array}{c}\text { Asian/Pacific } \\
\text { Islander }\end{array}$ & Hispanic \\
\hline West & $<5$ & $<5$ & 8 & $<5$ \\
Central & $<5$ & 6 & 14 & 10 \\
Northeast & 5 & 6 & 19 & 6 \\
Metro Boston & 21 & 20 & 21 & 8 \\
Boston & 8 & 26 & 19 & 8 \\
\hline Southeast & 14 & 9 & $<5$ & \\
\hline
\end{tabular}

\section{Percent of Confirmed Tuberculosis Cases by EOHHS Region Massachusetts: $2006(\mathrm{~N}=\mathbf{2 5 9})$}

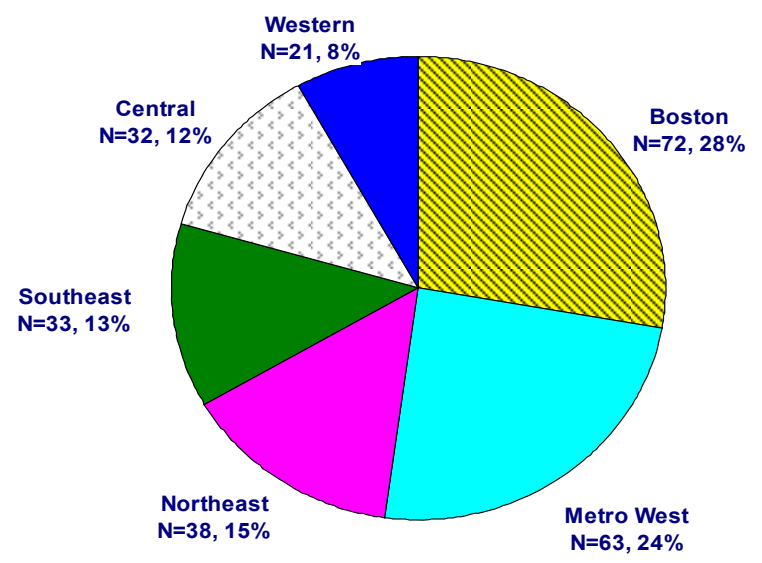




\section{Percent of Confirmed Tuberculosis Cases by Race/Ethnicity and EOHHS Region Massachusetts: 2006}
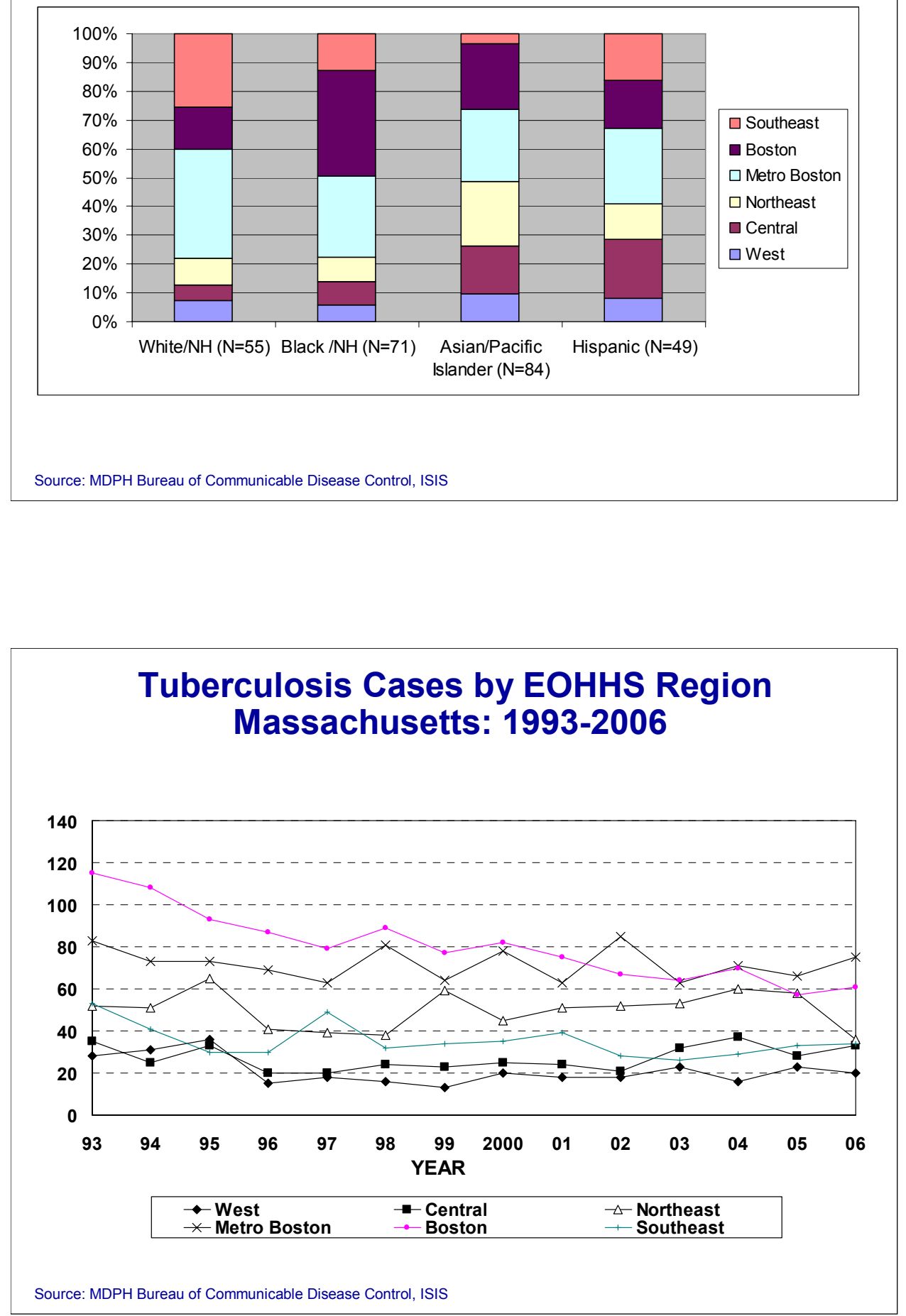


\section{Percent of Confirmed Tuberculosis Cases by Race/Ethnicity and EOHHS Region Massachusetts: 2006}
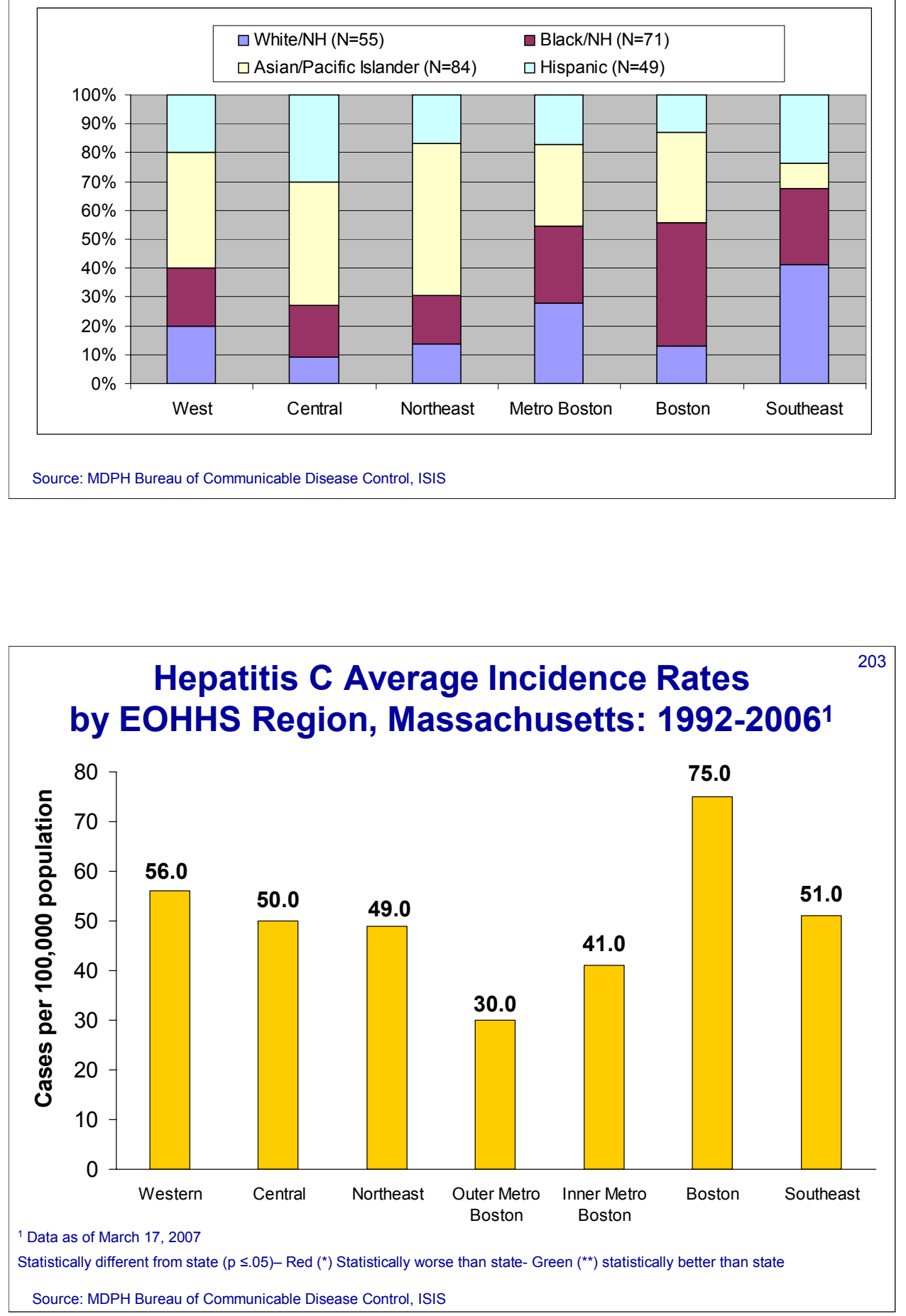

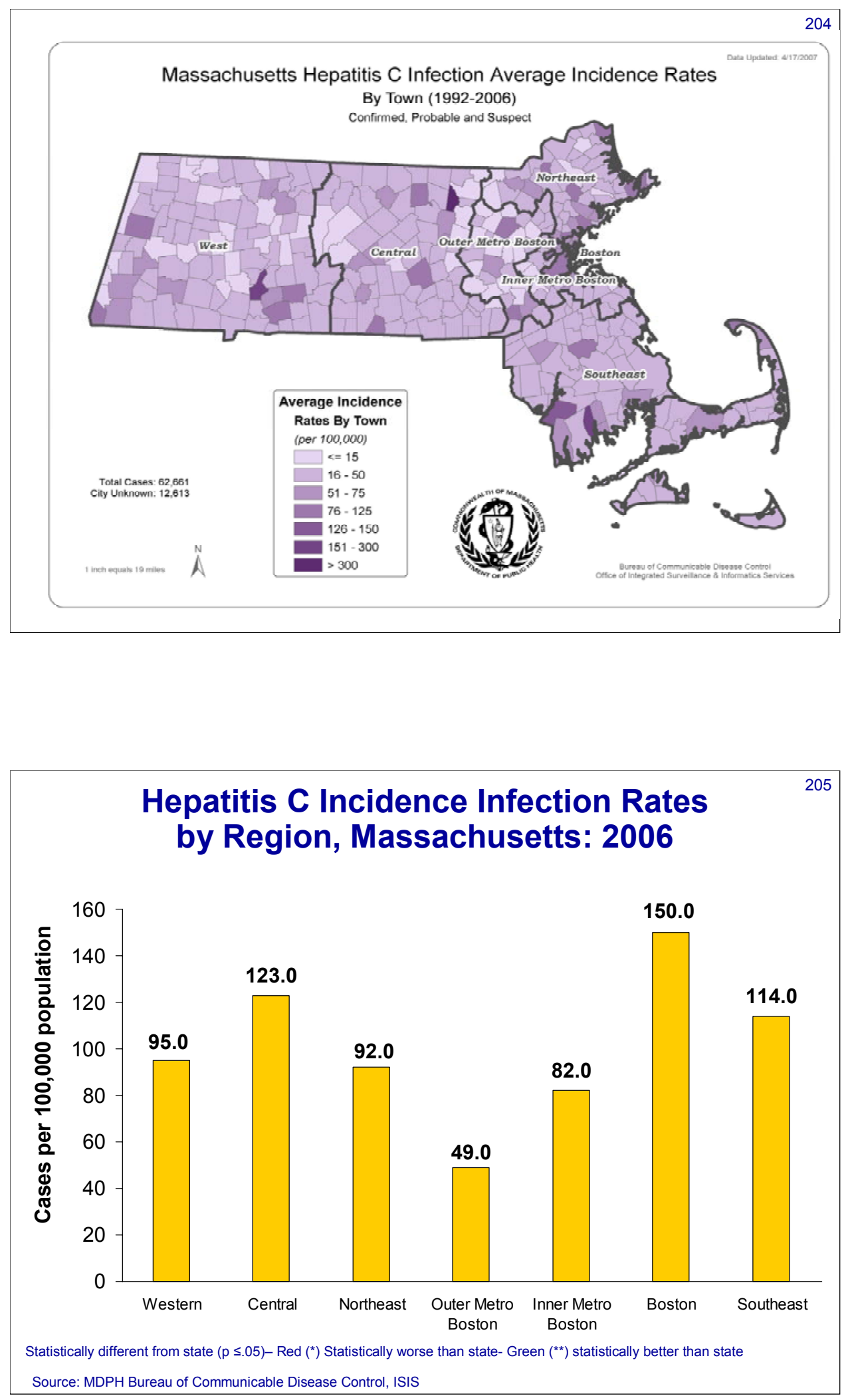

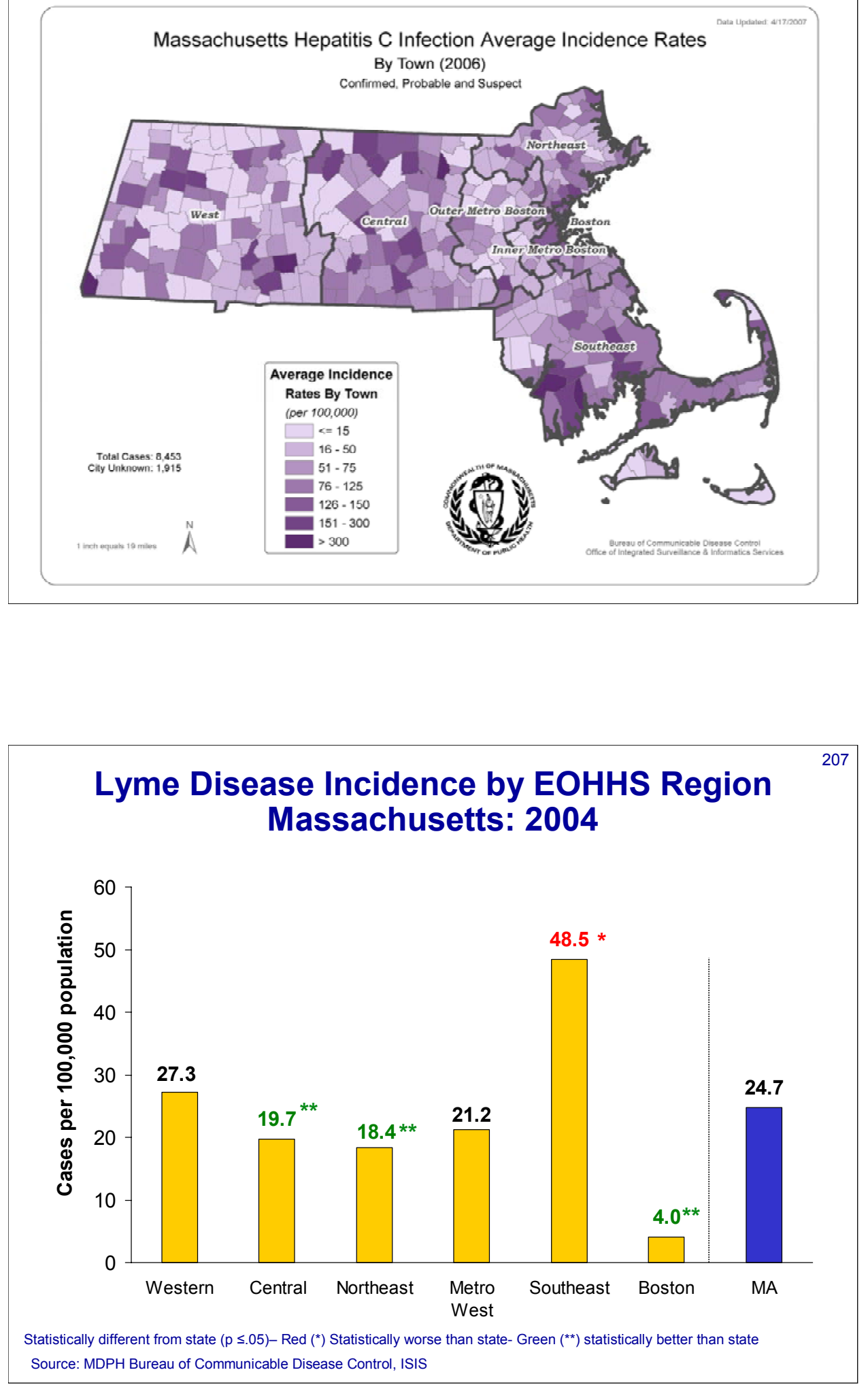

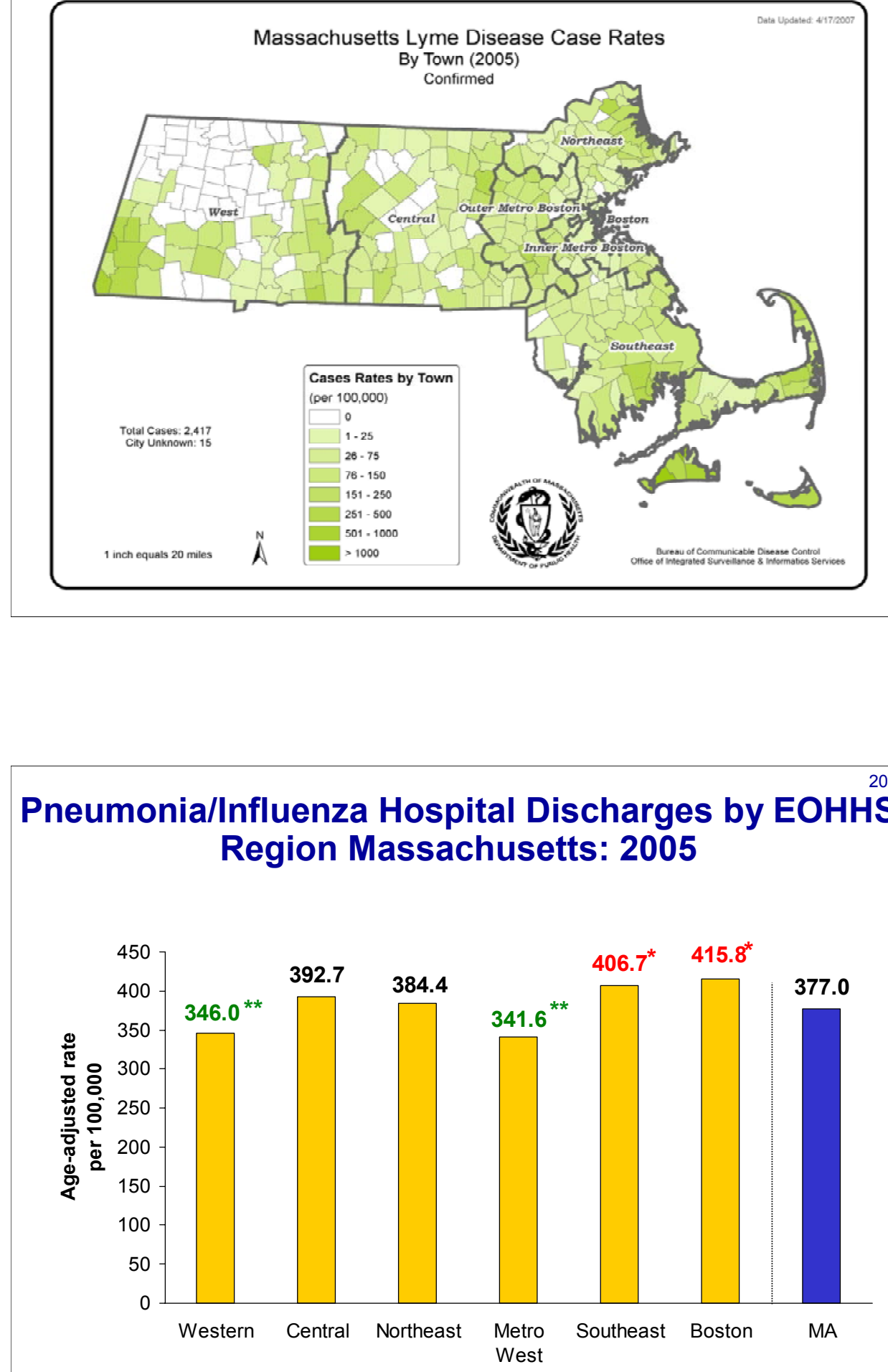

Statistically different from state $(p \leq .05)-\operatorname{Red}\left({ }^{*}\right)$ Statistically worse than state- Green $\left(^{* *}\right)$ statistically better than state Source: Division of Health Care Finance and Policy. Calendar Year 2005. Inpatient Hospital Discharge Database 


\section{Hospital Discharge Rates for Pneumonia/Influenza Cities in Boston Region \& Massachusetts: 2003-2005}

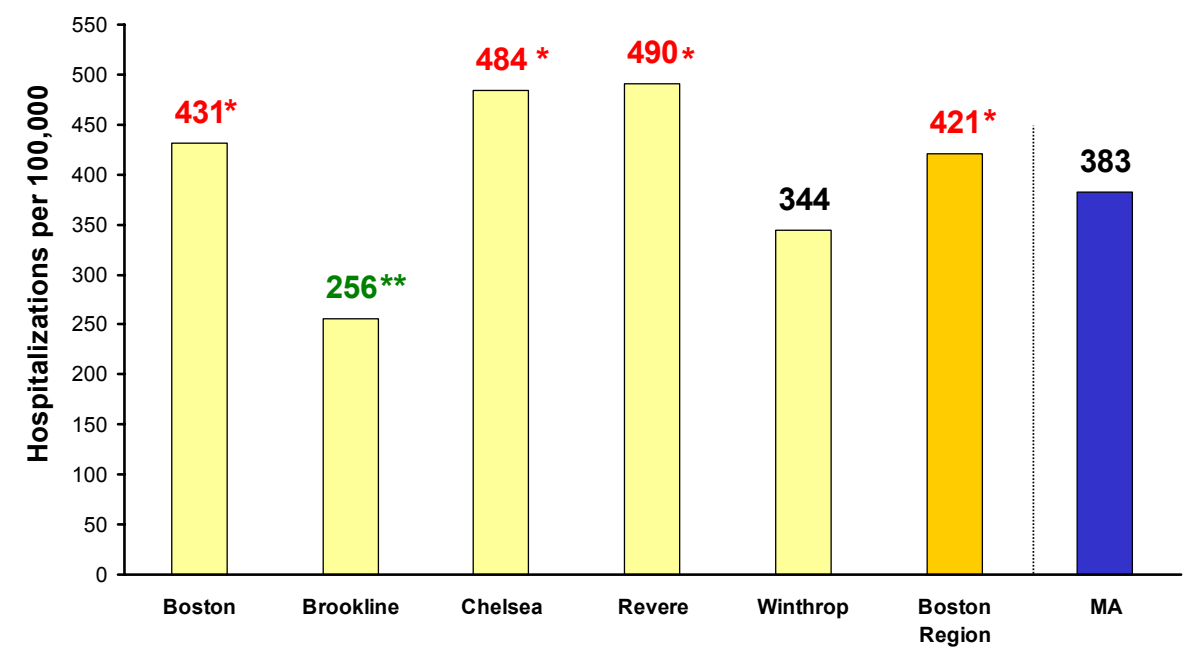

Statistically different from state $(p \leq .05)-\operatorname{Red}\left({ }^{*}\right)$ Statistically worse than state- Green $\left(^{* \star}\right)$ statistically better than state

\begin{tabular}{|c|c|c|c|c|c|c|c|}
\hline \multicolumn{8}{|c|}{$\begin{array}{c}\text { Summary Infectious Disease Indicators } \\
\text { by EOHHS Region }\end{array}$} \\
\hline Indicator & $\begin{array}{l}\text { MA } \\
\text { Total }\end{array}$ & Western & Central & $\begin{array}{c}\text { North } \\
\text { East }\end{array}$ & $\begin{array}{l}\text { Metro } \\
\text { West }\end{array}$ & Southeast & Boston \\
\hline $\begin{array}{l}\text { Chlamydia Incidence } \\
\text { (crude rates, 2005) }\end{array}$ & 225.7 & 369.4 & 145.4 & 178.0 & 106.1 & 169.7 & 588.5 \\
\hline $\begin{array}{l}\text { Gonorrhea Incidence } \\
\text { (crude rates, 2005) }\end{array}$ & 39.6 & 55.9 & 16.6 & 22.4 & 20.3 & 35.4 & 126.2 \\
\hline $\begin{array}{l}\text { Syphilis Incidence } \\
\text { (crude rates, 2005) }\end{array}$ & 6.3 & 3.1 & 1.7 & 4.9 & 4.7 & 2.7 & 22.7 \\
\hline $\begin{array}{c}\text { Tuberculosis Incidence } \\
\text { (crude rates, 2006) }\end{array}$ & 4.1 & 2.5 & 4.0 & 3.0 & 4.3 & 2.7 & 9.6 \\
\hline $\begin{array}{l}\text { Lyme Disease Incidence } \\
\text { (crude rates, 2004) }\end{array}$ & 24.7 & 27.3 & 19.7 & 18.4 & 21.2 & 48.4 & 4.0 \\
\hline $\begin{array}{l}\text { Average Hep C } \\
\text { Incidence Infection } \\
\text { (crude rates, 2006) }\end{array}$ & 114.0 & 95.0 & 123.0 & 92.0 & 49.0 & 82.0 & 150.0 \\
\hline $\begin{array}{l}\text { Pneumonia/ Influenza } \\
\text { Hospital Discharges } \\
\text { (age-adjusted, 2005) }\end{array}$ & 377.0 & 346.0 & 392.7 & 384.4 & 341.5 & 406.7 & 415.8 \\
\hline
\end{tabular}


ASTHMA 
Asthma Prevalence for School Children, Grades K-8 by Community Health Network Areas (CHNA) within EOHHS Regions Massachusetts Academic Year 2004-05
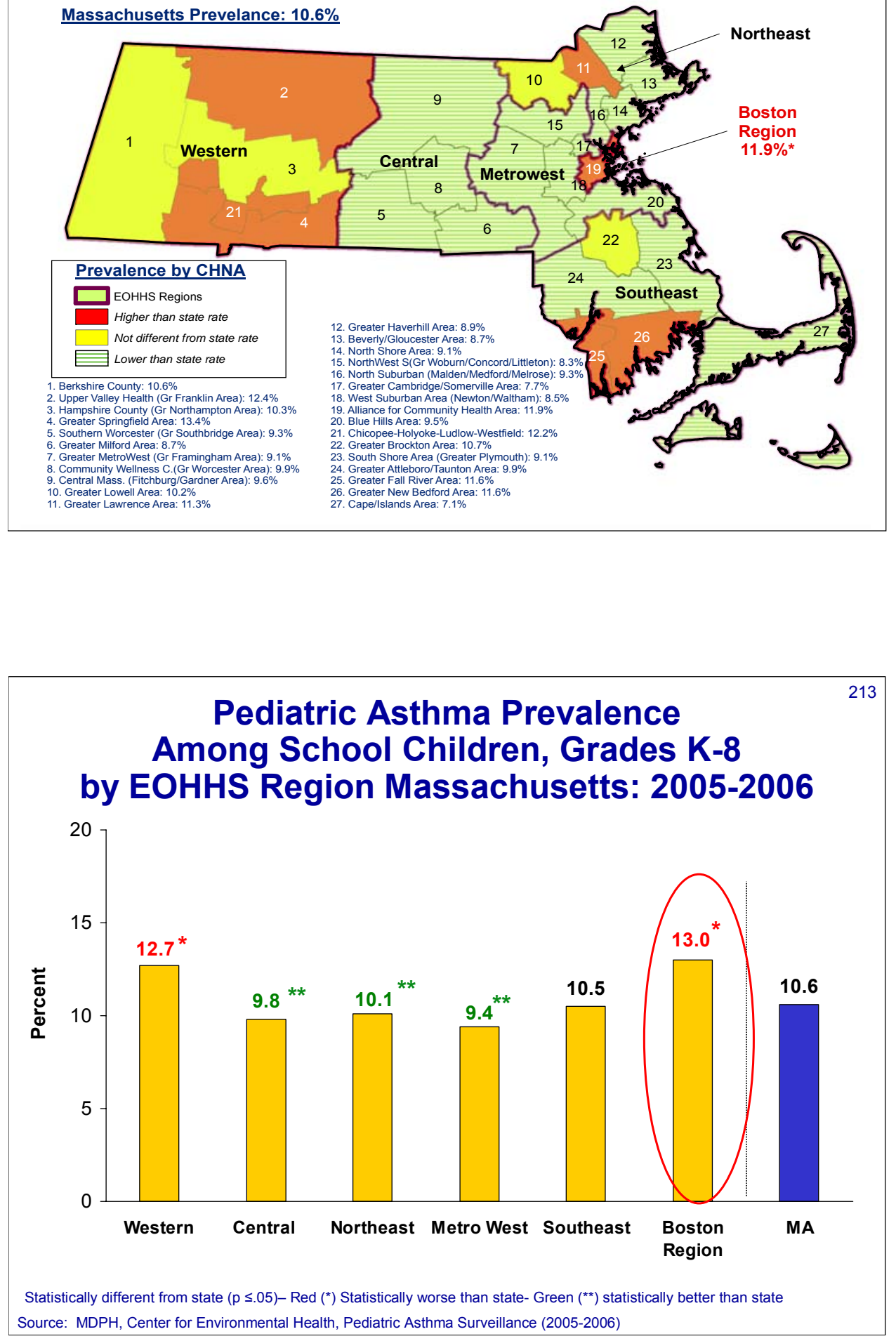


\section{Asthma Hospital Discharges \\ by EOHHS Region, Massachusetts: 2005}

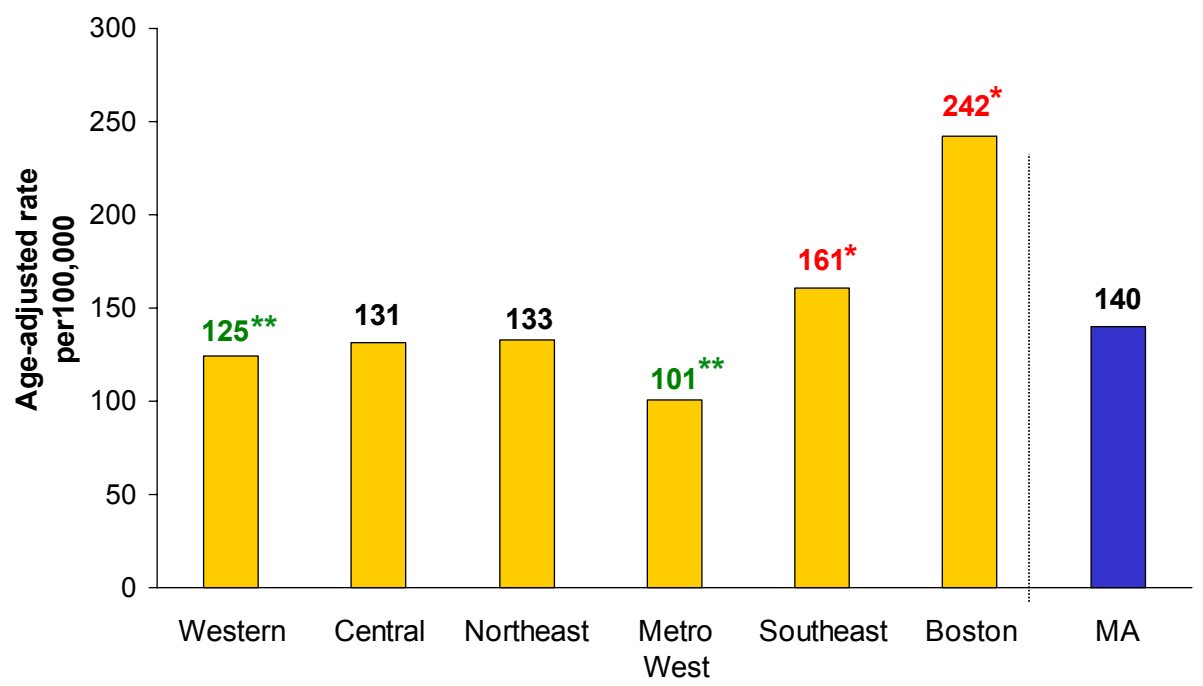

Statistically different from state $(\mathrm{p} \leq .05)-\operatorname{Red}\left({ }^{*}\right)$ Statistically higher than state- Green $\left(^{* *}\right)$ statistically lower than state

Source: Division of Health Care Finance and Policy. Calendar Year 2005. Inpatient Hospital Discharge Database

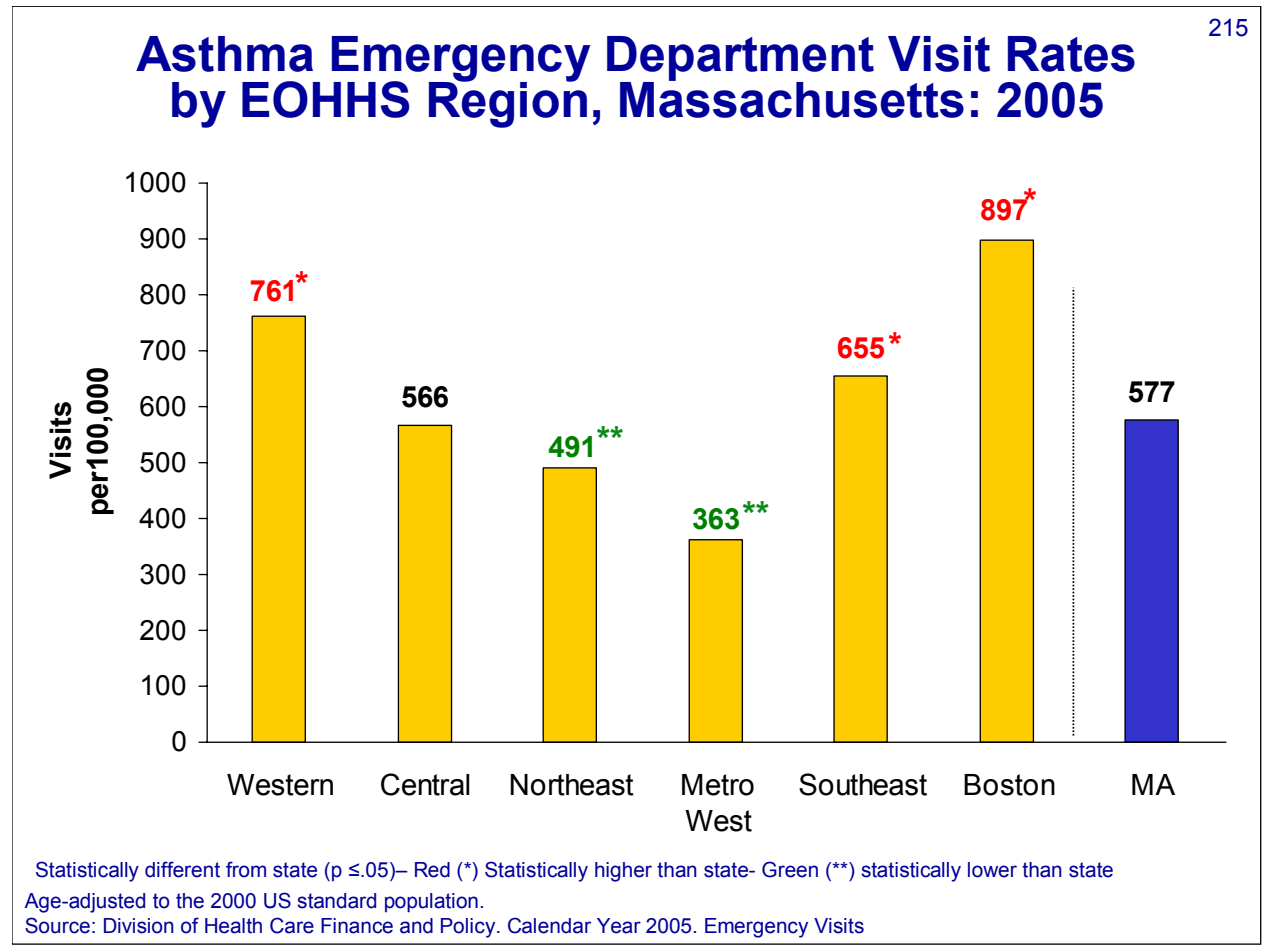


Emergency Department Visit Rates for Asthma by Race/Ethnicity Boston Region and Massachusetts: 2005

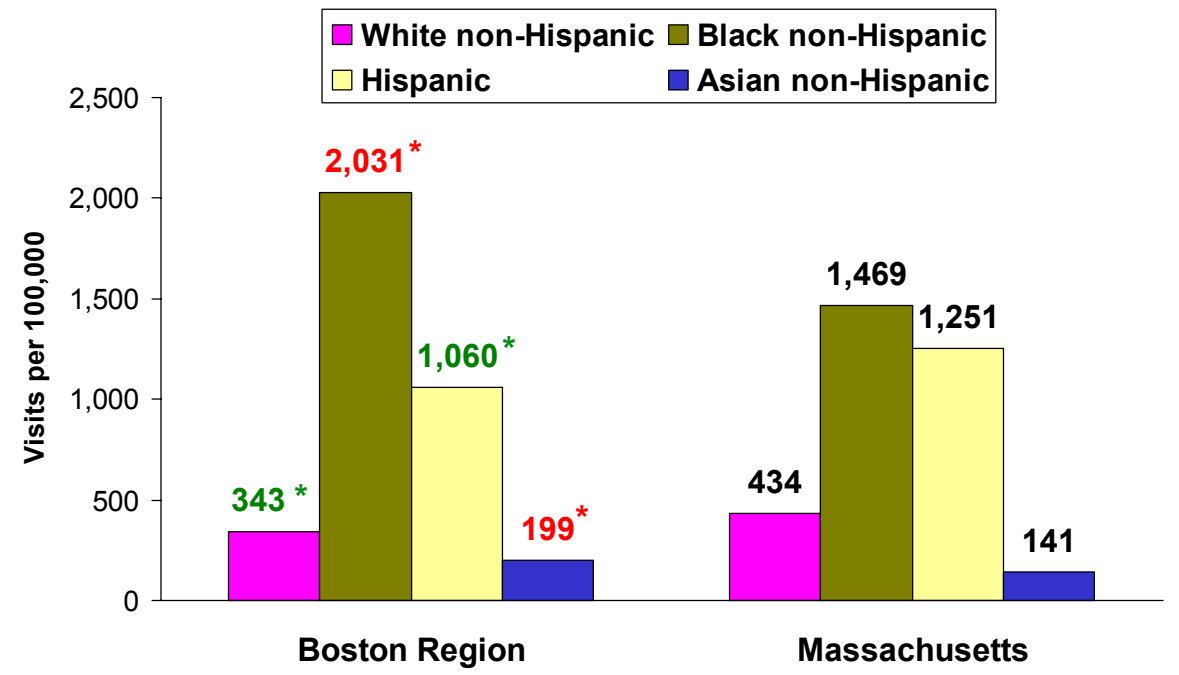

Statistically different from state $(p \leq .05)-\operatorname{Red}\left({ }^{*}\right)$ Statistically higher than state- Green $\left(^{* \star}\right)$ statistically lower than state Age-adjusted to the 2000 US standard population.

Source: Division of Health Care Finance and Policy. Calendar Year 2005. Emergency Department Visits

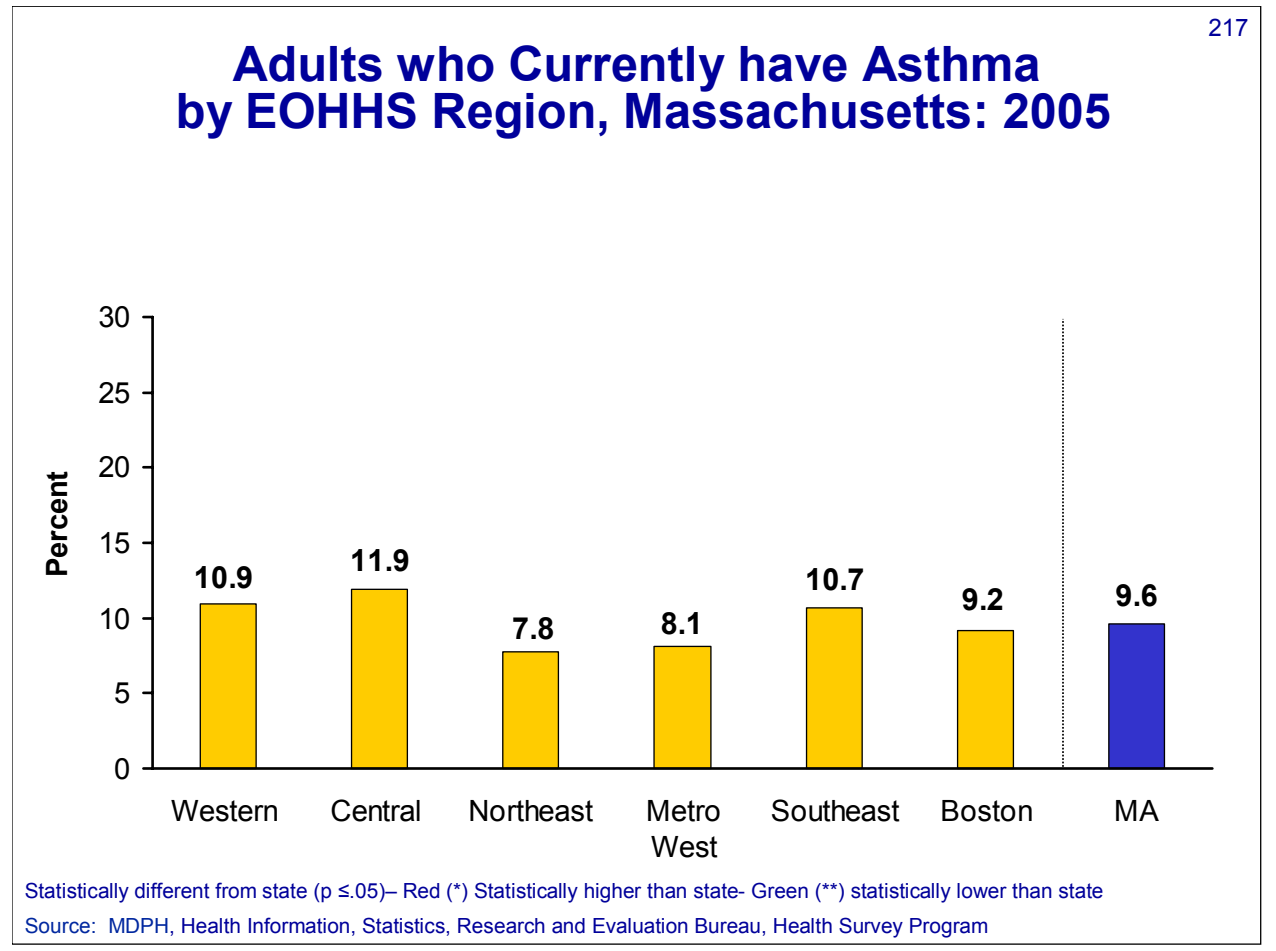


Asthma Emergency Department Visits, Children Ages 0-4

by EOHHS Region, Massachusetts: 2005

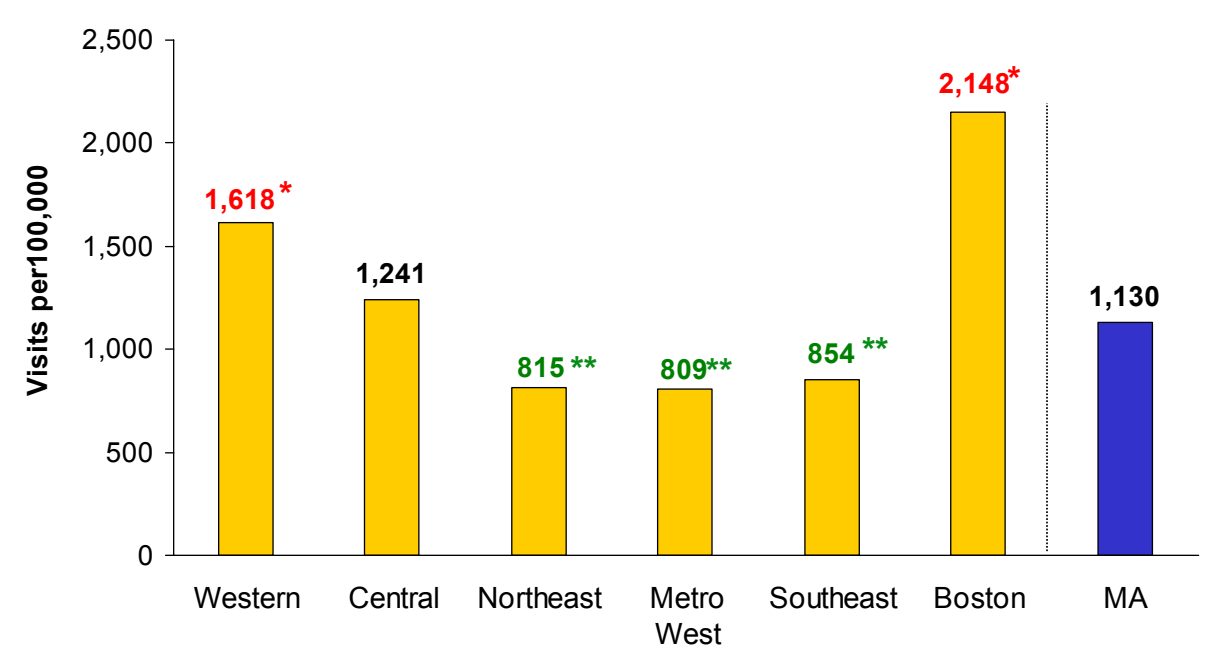

Statistically different from state $(p \leq .05)-\operatorname{Red}\left({ }^{*}\right)$ Statistically higher than state- Green $\left(^{* \star}\right)$ statistically lower than state

Source: Division of Health Care Finance and Policy. Calendar Year 2005. Emergency Visits

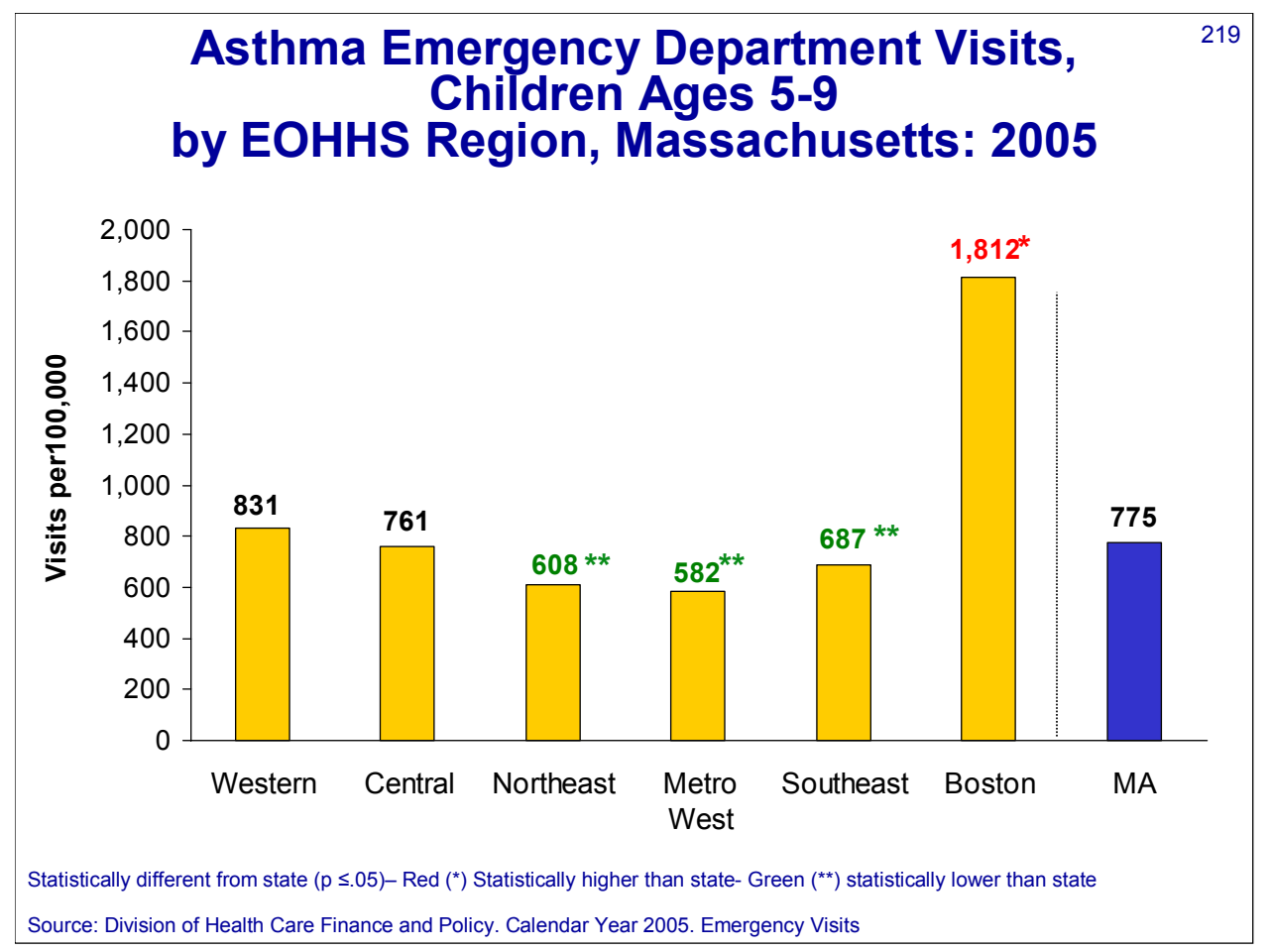




\section{Asthma Emergency Department Visits, Children Ages 10-14 \\ by EOHHS Region, Massachusetts: 2005}

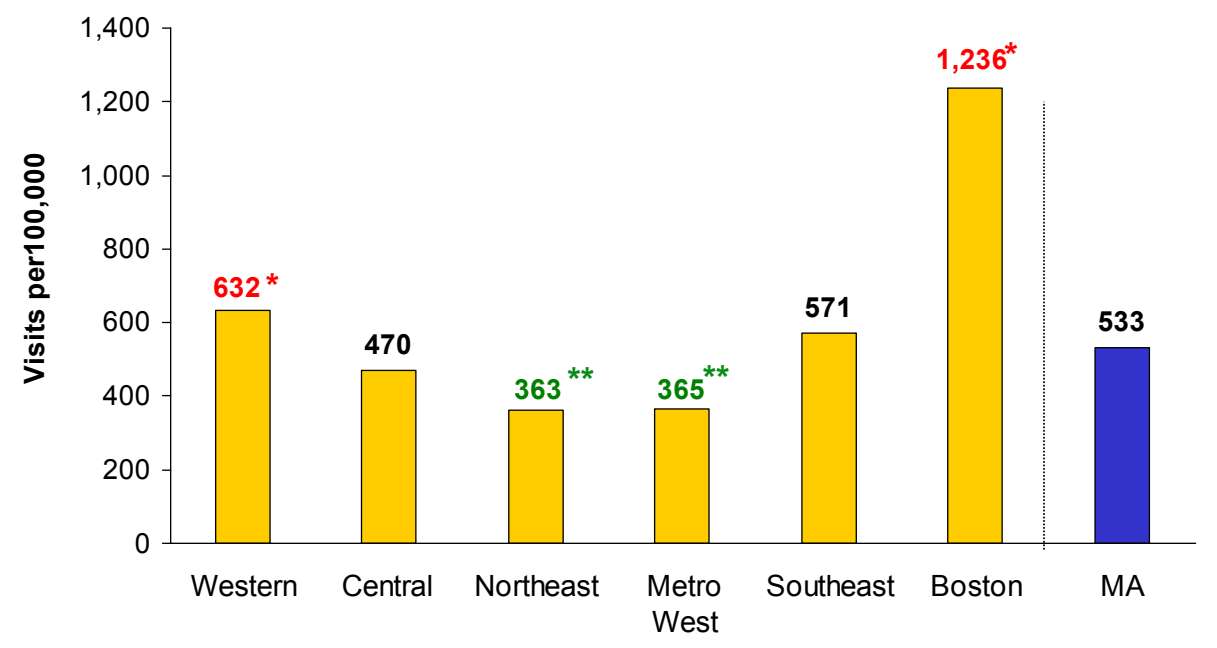

Statistically different from state $(p \leq .05)-\operatorname{Red}\left({ }^{*}\right)$ Statistically higher than state- Green $\left(^{* *}\right)$ statistically lower than state

Source: Division of Health Care Finance and Policy. Calendar Year 2005. Emergency Visits

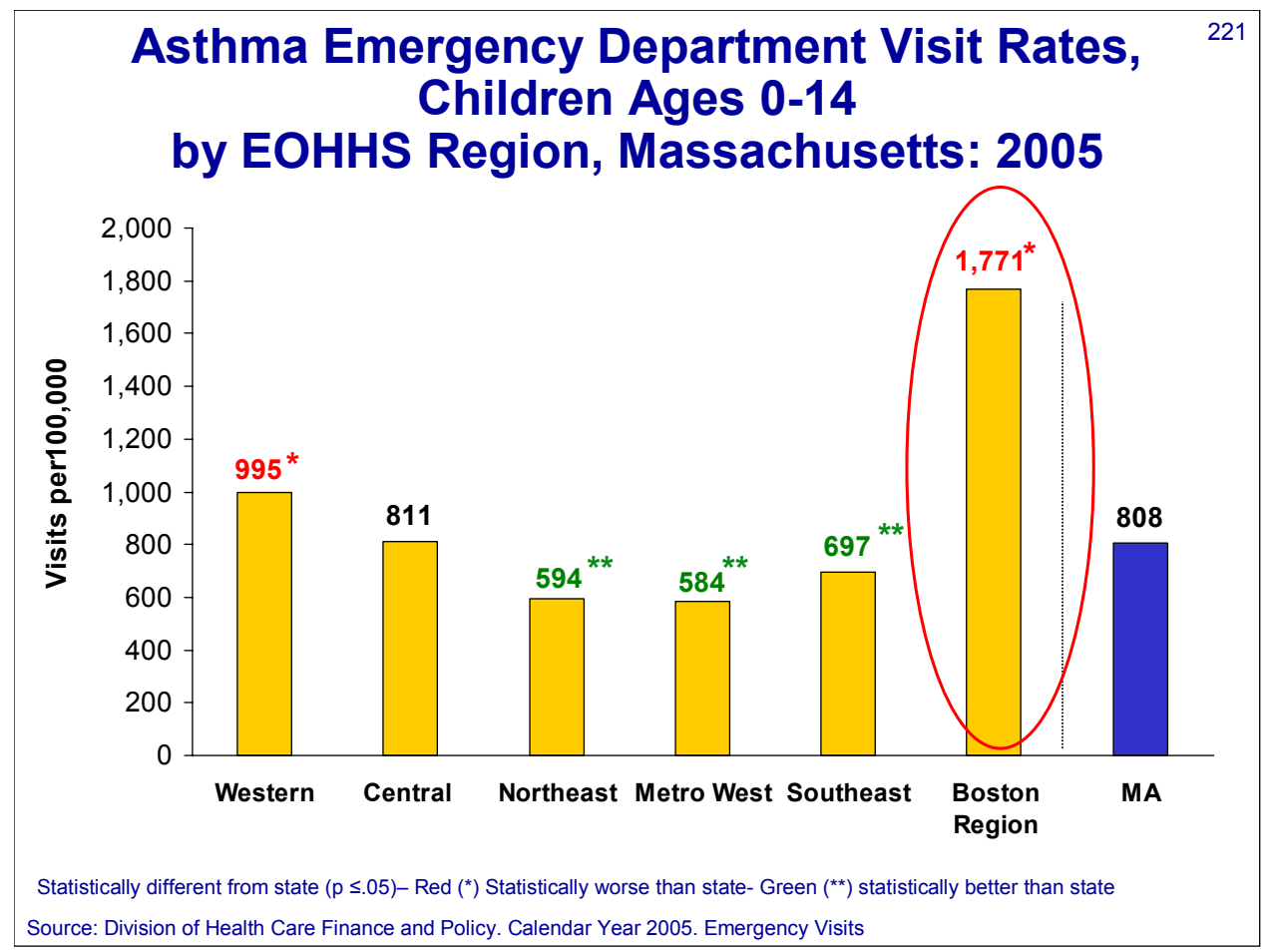


Asthma Emergency Department Visit Rates, Children Ages 0-14 Boston Region and Massachusetts: 2005
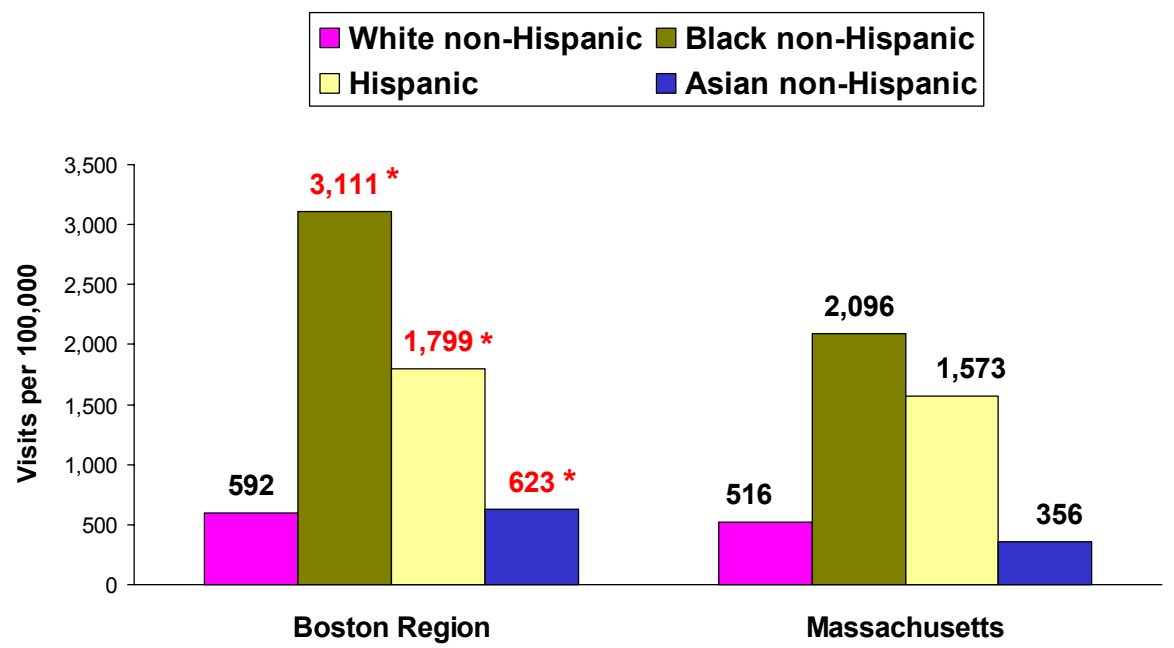

Statistically different from state $(p \leq .05)-\operatorname{Red}\left({ }^{*}\right)$ Statistically higher than state- Green $\left(^{* *}\right)$ statistically lower than state Source: Division of Health Care Finance and Policy. Calendar Year 2005. Emergency Department Visits

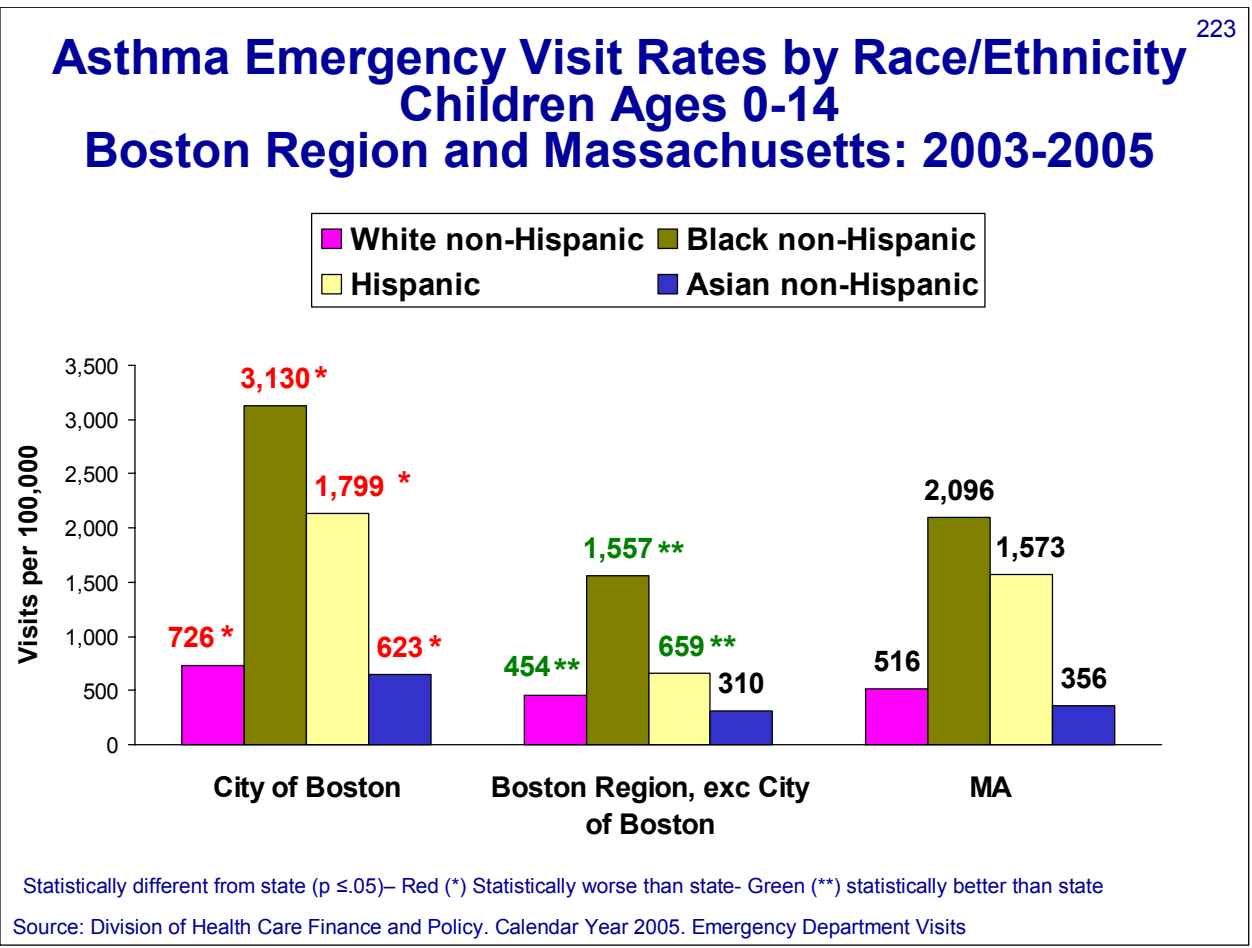




\section{Pediatric Asthma Prevalence Among School Children, Grades K-8 \\ by EOHHS Region Massachusetts: 2005-2006}

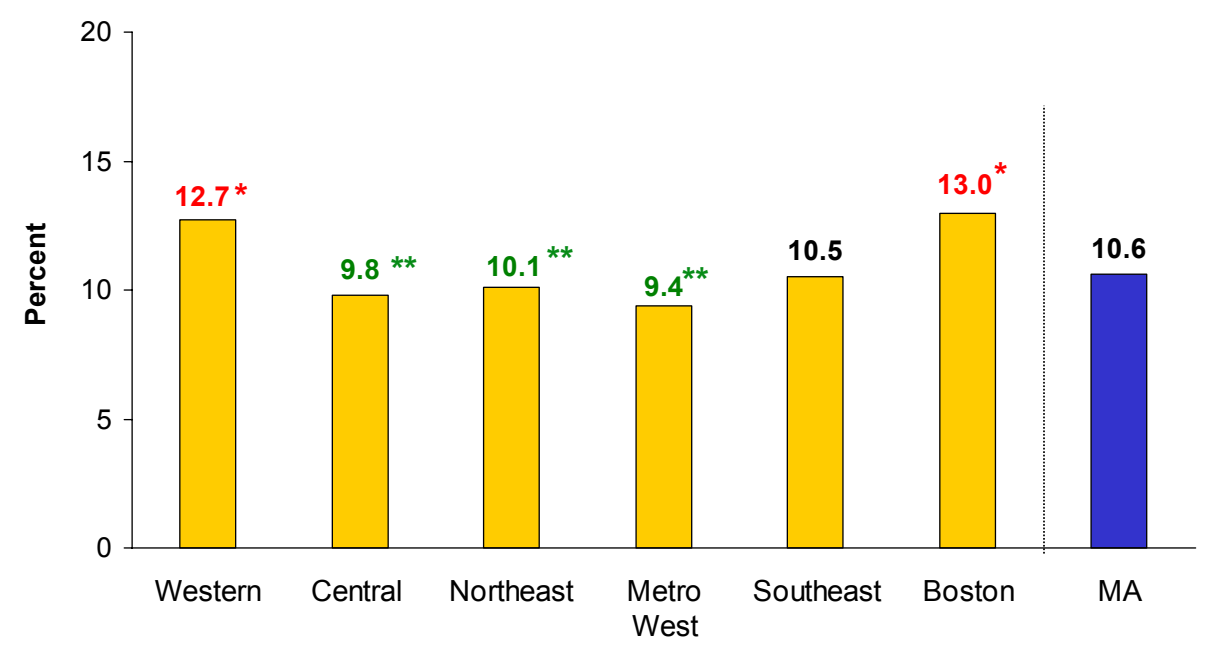

Statistically different from state $(p \leq .05)-\operatorname{Red}\left({ }^{*}\right)$ Statistically higher than state- Green $\left(^{* *}\right)$ statistically lower than state

Source: MDPH, Center for Environmental Health, Pediatric Asthma Surveillance (2005-2006)

Pediatric Asthma Emergency Visit Rates by Towns Among Children Ages <5

Boston Region - Massachusetts 2004-2005

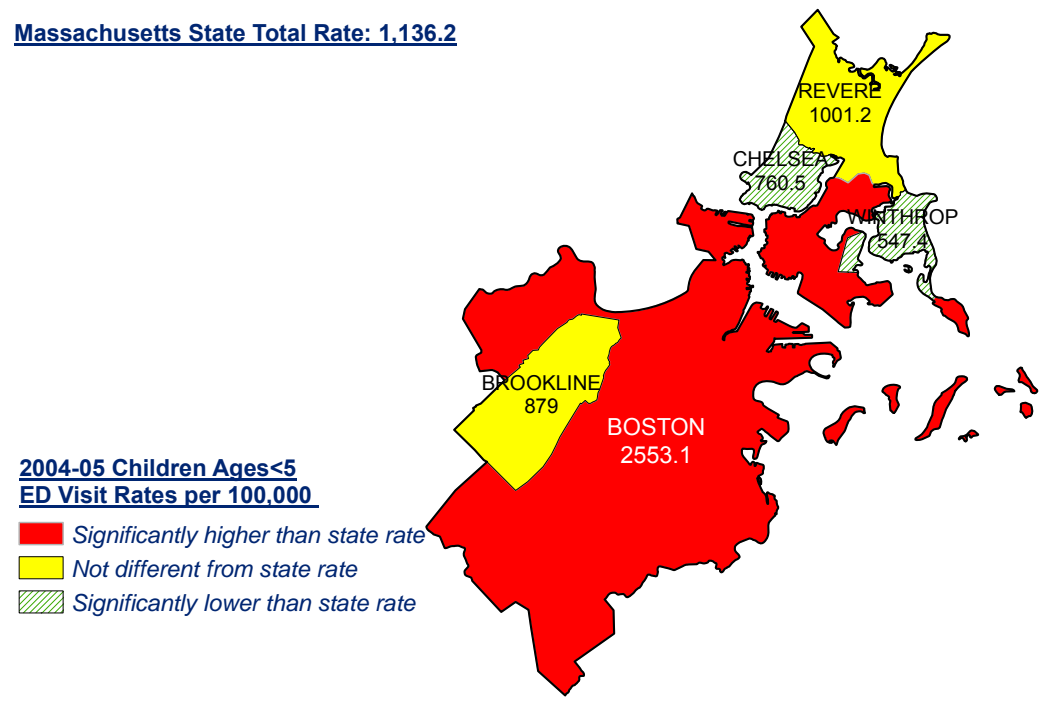

Source: Division of Health Care Finance and Policy. Calendar Year 2005. Emergency Visits 
Pediatric Asthma Hospitalization Rates by Towns Among Children Ages $<5$

Boston Region - Massachusetts 2004-2005

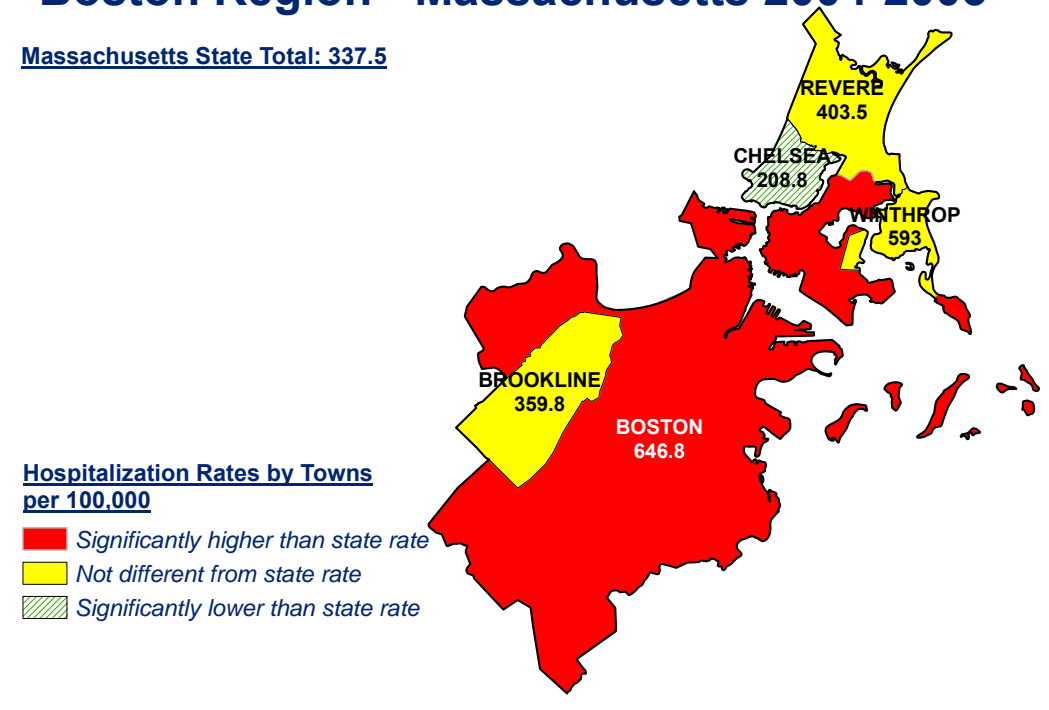

Source: Division of Health Care Finance and Policy. Calendar Year 2005. Hospital Discharges

\section{Summary Asthma Indicators by EOHHS Region, Massachusetts: 2005}

\begin{tabular}{|c|c|c|c|c|c|c|c|}
\hline Indicator & $\begin{array}{c}\text { MA } \\
\text { Total }\end{array}$ & Western & Central & Northeast & $\begin{array}{c}\text { Metro } \\
\text { West }\end{array}$ & $\begin{array}{c}\text { South } \\
\text { East }\end{array}$ & Boston \\
\hline $\begin{array}{c}\text { Asthma Hospital } \\
\text { Discharges (age- } \\
\text { adjusted per100,000) }\end{array}$ & $\mathbf{1 4 0 . 1}$ & 125.0 & 131.5 & 132.7 & 100.9 & 160.9 & 241.9 \\
\hline $\begin{array}{c}\text { Asthma ER Visits } \\
\text { (age-adjusted } \\
\text { per100,000) }\end{array}$ & $\mathbf{5 7 6 . 9}$ & 760.9 & 565.9 & 491.4 & 363.0 & 654.5 & 897.1 \\
$\begin{array}{c}\text { \% Adults who } \\
\text { currently have } \\
\text { asthma (2005) }\end{array}$ & $\mathbf{9 . 6}$ & 10.9 & 11.9 & 7.8 & 8.1 & 10.7 & 9.2 \\
$\begin{array}{c}\text { Asthma ER Visits } \\
\begin{array}{c}\text { Ages 0-14 (age- } \\
\text { adjusted per100,000) }\end{array}\end{array}$ & $\mathbf{8 0 8}$ & 995 & 811 & 594 & 584 & 697 & 1,771 \\
\hline
\end{tabular}




\section{Summary Asthma Indicators \\ by EOHHS Region, Massachusetts: 2005}

\begin{tabular}{||c|c|c|c|c|c|c|c|}
\hline Indicator & $\begin{array}{c}\text { MA } \\
\text { Total }\end{array}$ & Western & Central & Northeast & $\begin{array}{c}\text { Metro } \\
\text { West }\end{array}$ & $\begin{array}{c}\text { South } \\
\text { East }\end{array}$ & Boston \\
\hline $\begin{array}{c}\text { Asthma ER Visits } \\
\text { Ages 0-4 } \\
\text { (age adjusted } \\
\text { per100,000) }\end{array}$ & $\mathbf{1 , 1 3 0}$ & 1,618 & 1,241 & 815 & 809 & 854 & 2,148 \\
\hline $\begin{array}{c}\text { Asthma ER Visits } \\
\text { Ages 5-9 } \\
\text { (age adjusted } \\
\text { per100,000) }\end{array}$ & $\mathbf{7 7 5}$ & 831 & 761 & 608 & 582 & 687 & 1,812 \\
\hline $\begin{array}{c}\text { Asthma ER Visits } \\
\text { Ages 10-14 } \\
\text { (age adjusted } \\
\text { per100,000) }\end{array}$ & $\mathbf{5 3 3}$ & 632 & 470 & 363 & 365 & 571 & 1,236 \\
\hline $\begin{array}{c}\text { Pediatric Asthma } \\
\text { Prevalence } \\
\text { (2005-2006) }\end{array}$ & $\mathbf{1 0 . 6}$ & 12.7 & 9.8 & 10.1 & 9.4 & 10.5 & 13.0 \\
\hline
\end{tabular}




\section{OTHER HEALTH INDICATORS}


Total Cases of Elevated Blood Lead Levels (>=15 $\mu \mathrm{g} / \mathrm{dL}$ ) by EOHHS Region Massachusetts: 2005

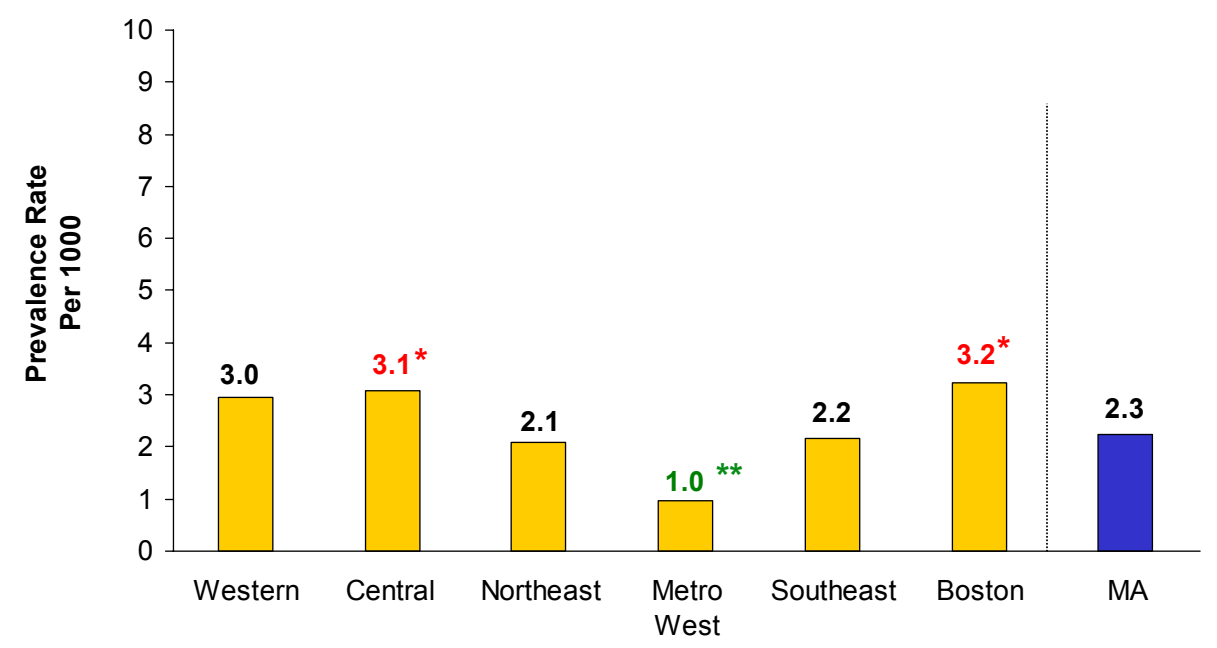

Statistically different from state $(p \leq .05)-\operatorname{Red}\left({ }^{*}\right)$ Statistically worse than state- Green $\left(^{* *}\right)$ statistically better than state Source: MDPH, Childhood Lead Poisoning Prevention Program (CLPPP)

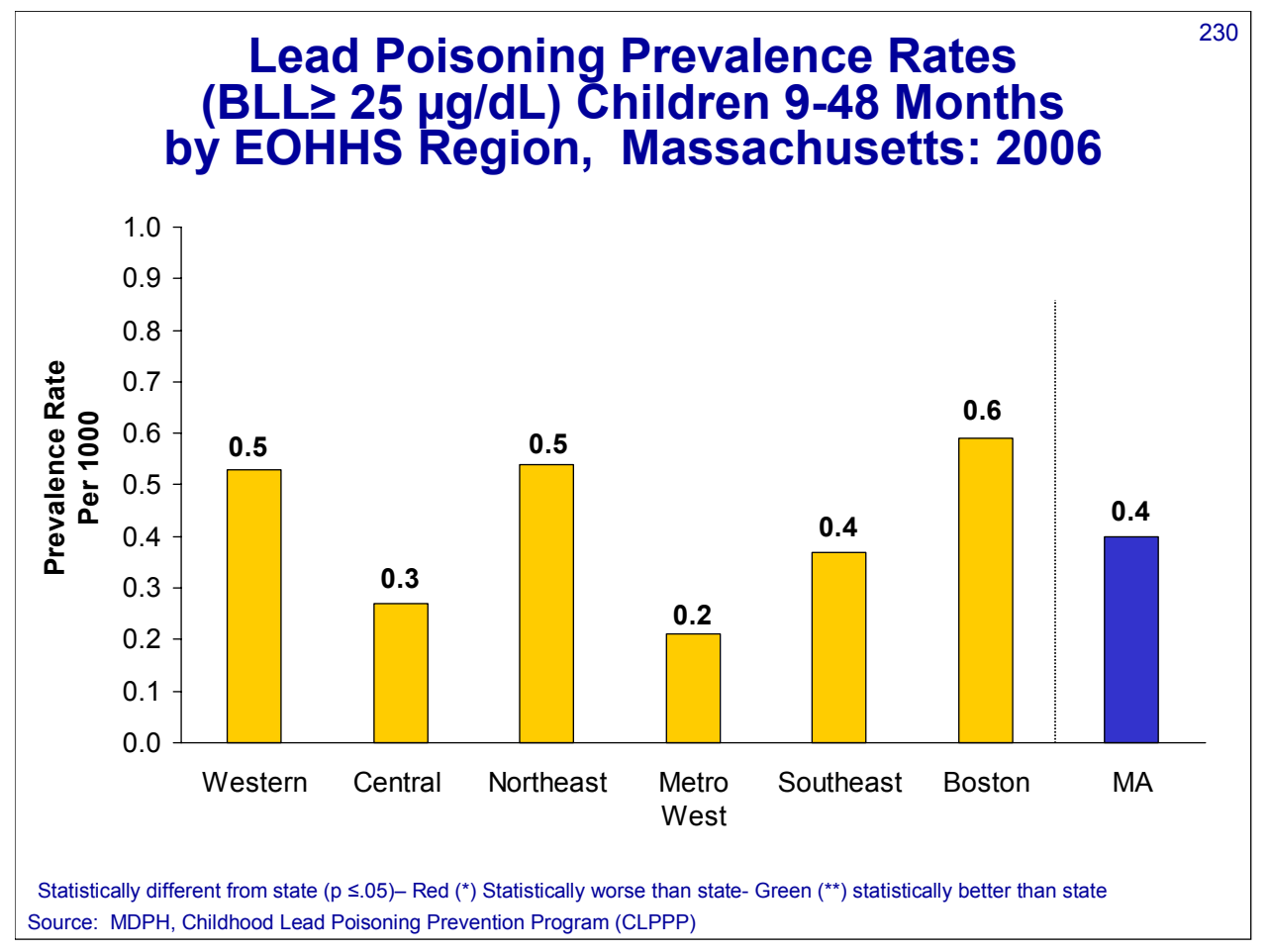




\section{Adults who Report Experiencing Intimate Partner ${ }^{231}$ Violence by EOHHS Region and Gender Massachusetts: 2005}

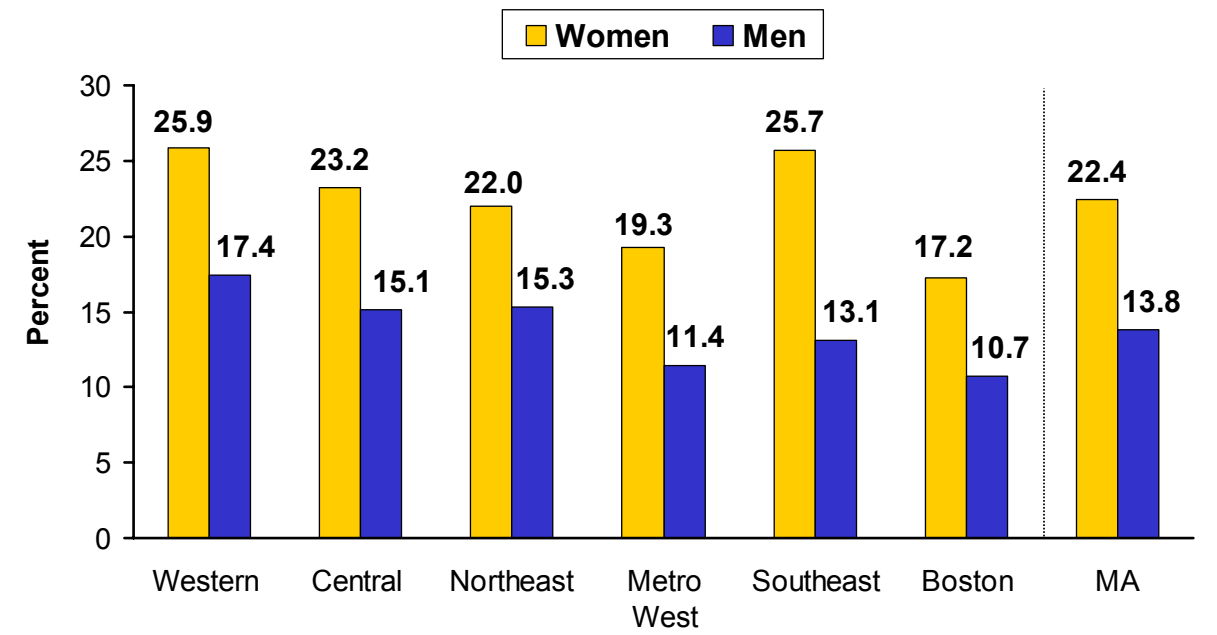

Statistically different from state $(p \leq .05)-\operatorname{Red}\left({ }^{*}\right)$ Statistically worse than state- Green $\left(^{* *}\right)$ statistically better than state Source: MDPH, Health Information, Statistics, Research and Evaluation Bureau, Health Survey Program

\section{Adults who Report Having a Disability by EOHHS Region, Massachusetts: 2005}

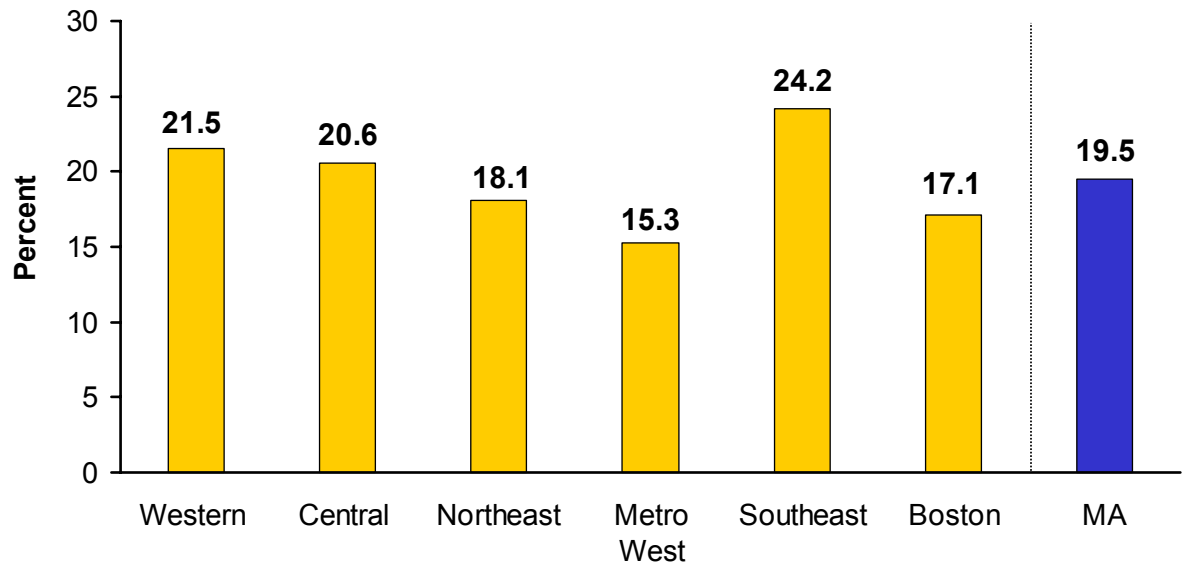


Adults who Report Having a Disability and needing help with activities

EOHHS Region, Massachusetts: 2005

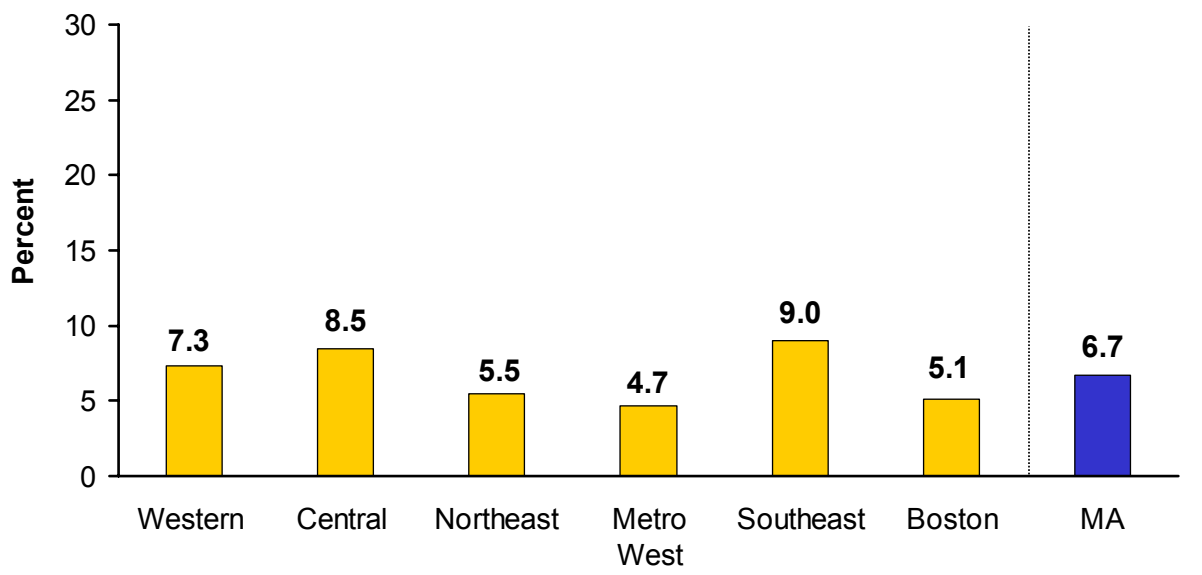

Statistically different from state $(p \leq .05)-\operatorname{Red}\left({ }^{*}\right)$ Statistically worse than state- Green $\left(^{* \star}\right)$ statistically better than state Source: MDPH, Health Information, Statistics, Research and Evaluation Bureau, Health Survey Program

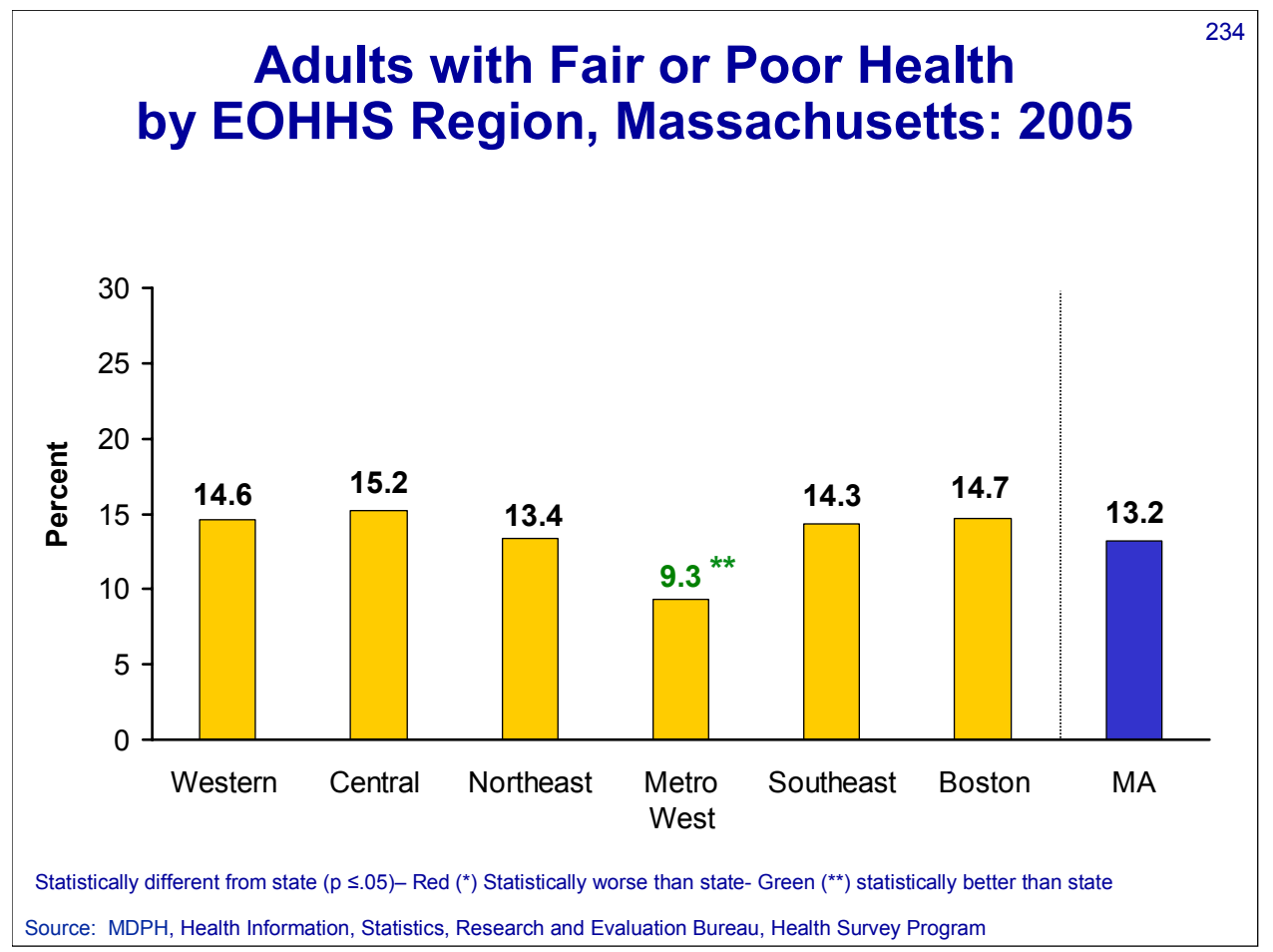




\section{Adults who Currently Report Fair or Poor Health ${ }^{235}$ by Race and Ethnicity Boston Region and Massachusetts: 2005}
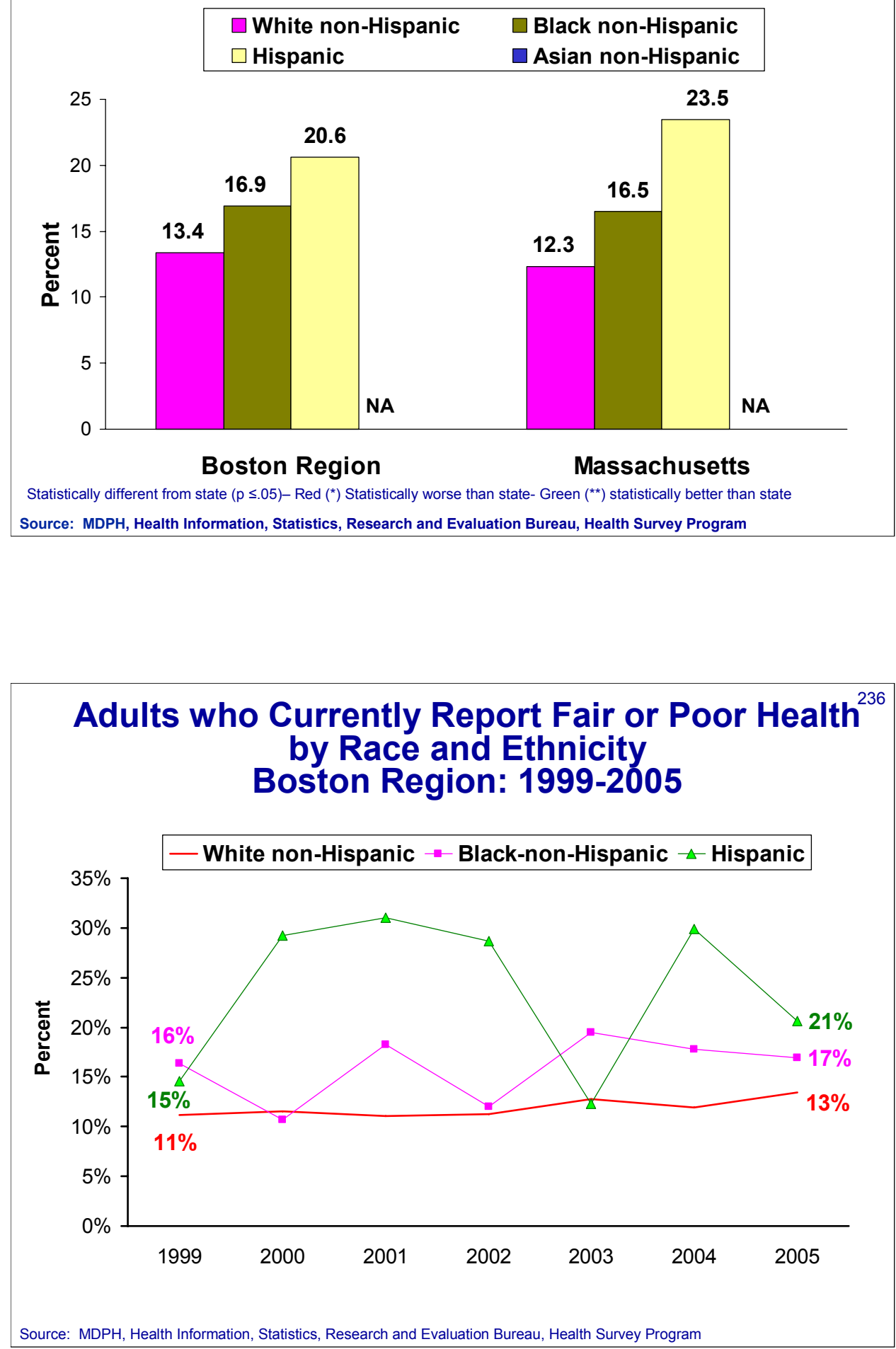


\section{Adults with Poor Mental Health for 15 or more days in Past Month by EOHHS Region Massachusetts: 2005}

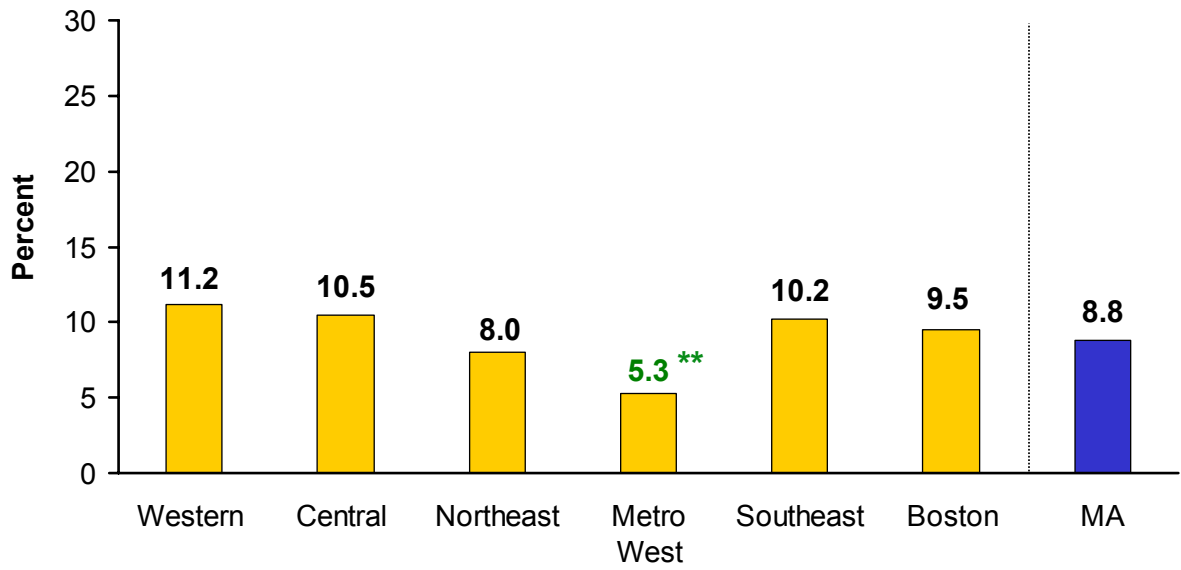

Statistically different from state $(p \leq .05)-$ Red $\left(^{*}\right)$ Statistically worse than state- Green $\left(^{* \star}\right)$ statistically better than state

Source: MDPH, Health Information, Statistics, Research and Evaluation Bureau, Health Survey Program

\begin{tabular}{|c|c|c|c|c|c|c|c|}
\hline \multicolumn{8}{|c|}{$\begin{array}{c}\text { Summary Other Health Indicators } \\
\text { by EOHHS Region }\end{array}$} \\
\hline Indicator & $\begin{array}{c}\text { MA } \\
\text { Total }\end{array}$ & Western & Central & $\begin{array}{c}\text { North } \\
\text { East }\end{array}$ & $\begin{array}{l}\text { Metro } \\
\text { West }\end{array}$ & $\begin{array}{l}\text { South } \\
\text { East }\end{array}$ & Boston \\
\hline $\begin{array}{l}\% \text { Fair or Poor } \\
\text { Health }(2005)\end{array}$ & 13.2 & 14.6 & 15.2 & 13.4 & 9.3 & 14.3 & 14.7 \\
\hline $\begin{array}{l}\text { Cases of } \\
\text { Elevated Blood } \\
\text { Lead levels } \\
\text { (Percent of All } \\
\text { Children Screened } \\
\text { Per 1000, 2005) }\end{array}$ & 2.3 & 2.9 & 3.1 & 2.1 & 1.0 & 2.2 & 3.2 \\
\hline $\begin{array}{l}\text { \% Visited Dentist } \\
\text { Past Year }(2004)\end{array}$ & 78.2 & 75.4 & 75.1 & 76.3 & 86.1 & 76.8 & 74.1 \\
\hline $\begin{array}{c}\% \text { 6+ Teeth } \\
\text { Missing (2004) }\end{array}$ & 15.1 & 18.8 & 15.4 & 16.5 & 10.5 & 17.4 & 13.7 \\
\hline $\begin{array}{l}\% \text { Poor Mental } \\
\text { Health for } 15 \text { or } \\
\text { more days in } \\
\text { Past Month }(2005) \\
\end{array}$ & 8.8 & 11.2 & 10.5 & 8.0 & 5.3 & 10.2 & 9.5 \\
\hline
\end{tabular}




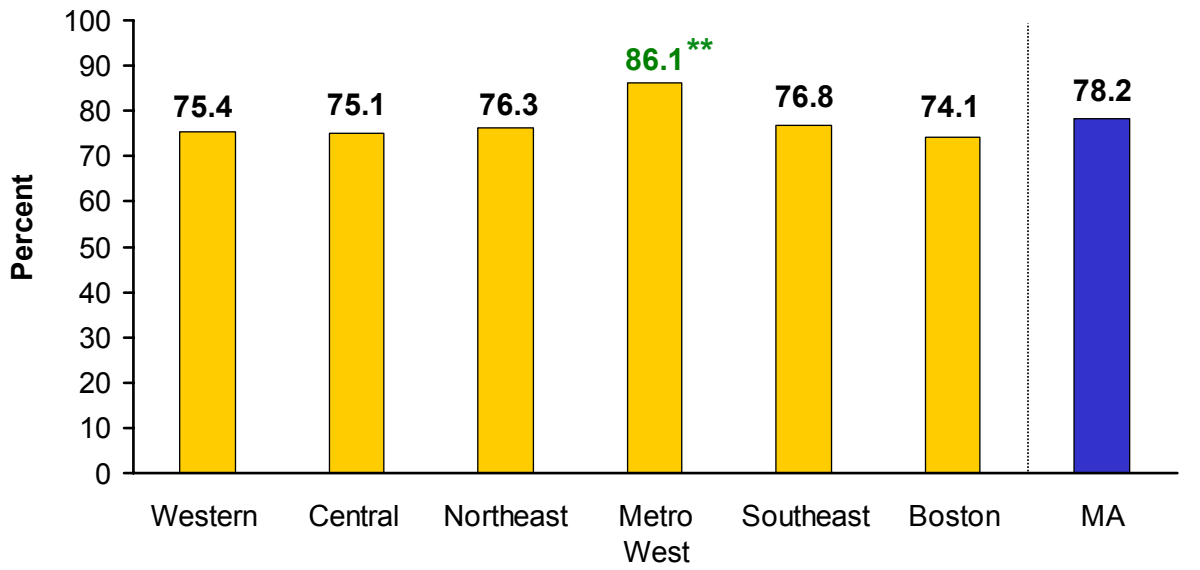

Statistically different from state $(p \leq .05)$ - Red $\left({ }^{*}\right)$ Statistically worse than state- Green $\left(^{* *}\right)$ statistically better than state Source: MDPH, Health Information, Statistics, Research and Evaluation Bureau, Health Survey Program

Adults who have lost 6 or more teeth to decay or disease by EOHHS Region, Massachusetts: 2004

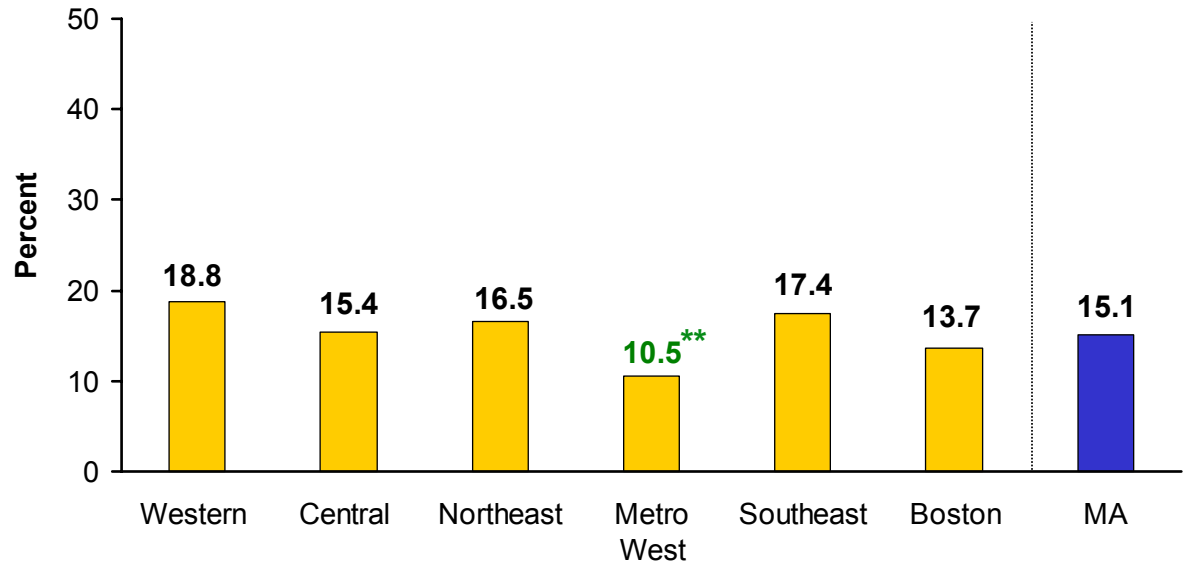

Statistically different from state $(p \leq .05)-\operatorname{Red}\left({ }^{*}\right)$ Statistically worse than state- Green $\left(^{\star *}\right)$ statistically better than state Source: MDPH, Health Information, Statistics, Research and Evaluation Bureau, Health Survey Program 
Refugee Arrivals: Boston Region FY02-FY06

- 2,317 refugees resettled over 5 years

- Great diversity in population

- $40 \%$ age $\leq 19$ at arrival

- Cities/Towns with largest numbers of arrivals: Boston, Chelsea, Cambridge, Revere
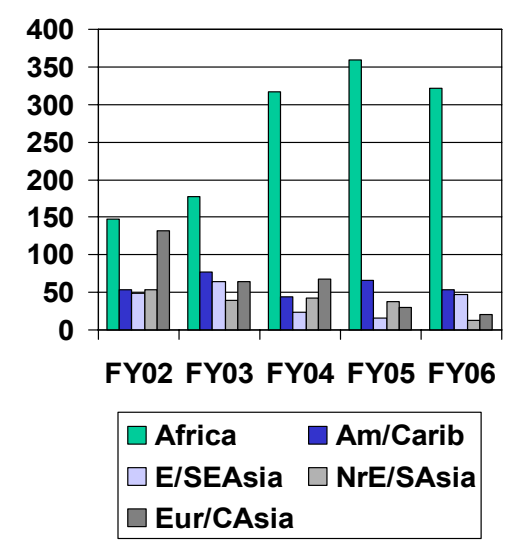

\section{Refugee Health Assessment Findings Boston Region: FY02-FY06}

- High rates of latent TB infection (LTBI)

- 2,093 refugees tested: $48 \%$ positive

- Intensive follow-up in partnership with TB clinics, local health and DPH to assure testing, evaluation and treatment

- Rapid assessment identifies active disease early to prevent spread

- Risk of lead poisoning among refugee children

- 60 refugee children identified with elevated blood lead levels at arrival $(20 \%$ of children $<7$ years old)

- Poor nutritional status puts children at risk of increased absorption of lead post-resettlement

- Need for bilingual, bicultural services to promote access to care 


\section{Summary Refugees by EOHHS Regions Massachusetts: 2002-2006}

\begin{tabular}{|c|c|c|c|c|}
\hline Indicator & Western & Central & North East & $\begin{array}{c}\text { Metro West } \\
\text { and Boston } \\
\text { Regions }\end{array}$ \\
\hline Refugee Arrivals & 2,204 & 858 & 1,312 & 2,843 \\
\hline Largest Group & $\begin{array}{l}\text { European and } \\
\text { Central Asian }\end{array}$ & African & $\begin{array}{l}\text { African and } \\
\text { South East } \\
\text { Asian }\end{array}$ & African \\
\hline $\mathbf{1 9}$ years or less & $44 \%$ & $51 \%$ & $45 \%$ & $39 \%$ \\
\hline Largest Cities & $\begin{array}{l}\text { Greenfield, } \\
\text { Springfield, West } \\
\text { Springfield, }\end{array}$ & Worcester & $\begin{array}{l}\text { Lowell, Lynn, } \\
\text { Malden }\end{array}$ & $\begin{array}{l}\text { Boston, } \\
\text { Cambridge, } \\
\text { Chelsea, Everett, } \\
\text { Quincy, Revere, } \\
\text { Somerville, } \\
\text { Waltham }\end{array}$ \\
\hline \%TB Positive & $41 \%$ & $33 \%$ & $44 \%$ & $47 \%$ \\
\hline
\end{tabular}

Source: MDPH, Refugee and Immigrant Health Program and the Bureau of Communicable Disease Control office of Integrated Surveillance and Informatics

\section{Premature Mortality Rate (PMR) 1}

- Definition

- deaths before age 75

- Rationale

- two-thirds of deaths to persons ages 75+

- vast majority of deaths to persons age $75+$ are due to chronic conditions associated with aging

- Focus on deaths to persons less than 75 years may be more preventable

- Summary health statistic

- excellent measure to reflect the health status of a population

- used as a health status indicator that can be used to focus prevention initiatives and target programs 


\section{Premature Mortality Rates (PMR) by Region Massachusetts: 2005}

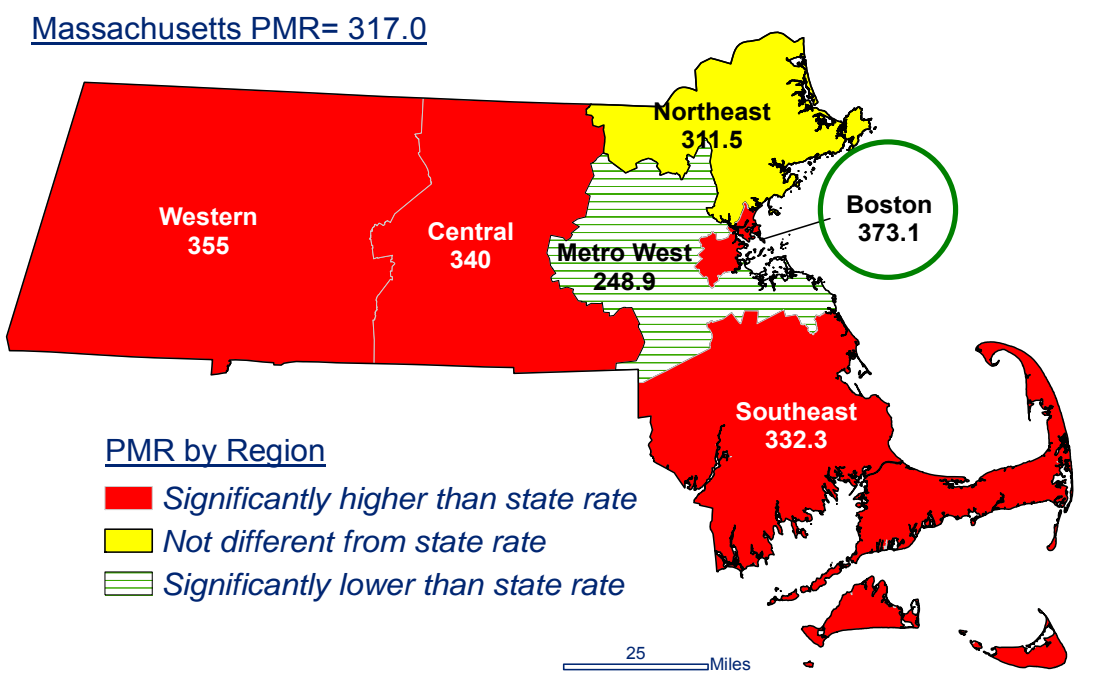

Rates are per 100,000 persons under 75 years of age, age-adjusted to the 2000 US standard population under 75 years of age. Source: Massachusetts Department of Public Health, Center for Health Information, Statistics, Research, and Evaluation

\section{PMR- Boston Region by City/Town Massachusetts: 2005}

Massachusetts PMR $=317.0$

Boston Region PMR= 373.1

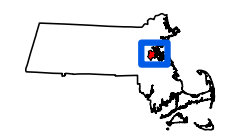

PMR by City/Town

Significantly higher than state rate

Not different from state rate

Significantly lower than state rate

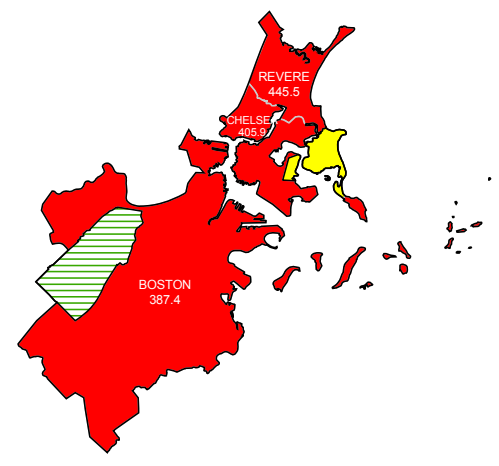

$\stackrel{2.5}{\longrightarrow}$ Miles

Rates are per 100,000 persons under 75 years of age, age-adjusted to the 2000 US standard population under 75 years of age. Source: Massachusetts Department of Public Health, Center for Health Information, Statistics, Research, and Evaluation 
Median Household Income and PMR by EOHHS Regions, Massachusetts: 2005

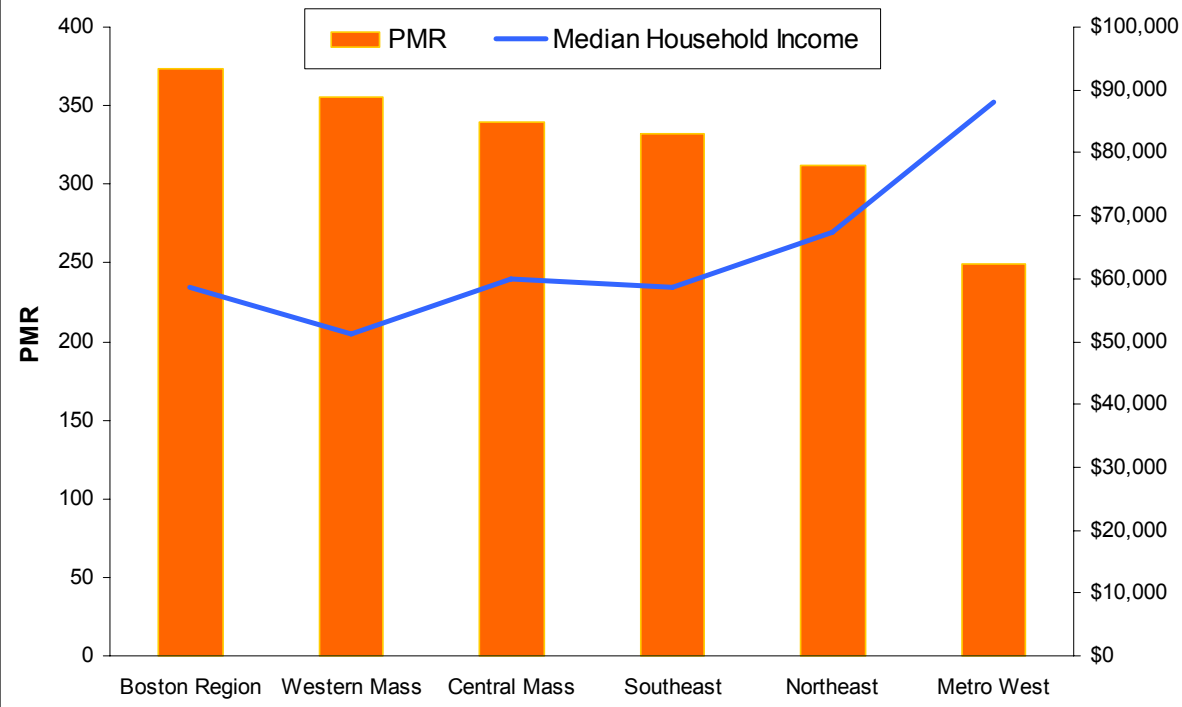

Source: MDPH, Health Information, Statistics, Research and Evaluation Bureau, Research \& Epidemiology Program State Median Household Income: \$66,365

\section{Premature Mortality Rate (PMR) by EOHHS Region: 2005}

\begin{tabular}{|l|c|}
\hline & PMR \\
\hline Massachusetts & $\mathbf{3 1 7 . 0}$ \\
\hline Western Region & 355.0 \\
\hline Central Region & 340.0 \\
\hline Northeast Region & 311.5 \\
\hline Metrowest Region & 248.9 \\
\hline Southeast Region & 332.3 \\
\hline Boston Region & 373.1 \\
\hline
\end{tabular}

Better Outcome (significantly different from state) 


\section{SUMMARY}




\section{Summary}

Residents of large cities are disproportionately affected by, including the following:

Boston: Teen pregnancies, diagnosis of prostate cancer \& HIV, hospitalizations for hypertension \& diabetes, admissions for substance abuse treatment, emergency visits of children with asthma, deaths to diabetes, opioidrelated fatal overdoses, firearm injuries, and homicides

Chelsea: Teen pregnancies, hospitalizations for hypertension and diabetes, deaths to diabetes and to opioid-related fatal overdoses

Revere: Teen pregnancies, hospitalizations for diabetes, admissions for substance abuse treatment, deaths to lung cancer and to opioid-related fatal overdoses

Winthrop: Deaths to lung cancer, admissions for heroin abuse treatment

\section{Health Disparities}

The following groups of the Boston region, when compared with those at the state level, have higher rates of:

Blacks: hospitalizations for hypertension \& diabetes, homicides, deaths to firearm injuries and HIV

Whites: hospitalizations for hypertension \& diabetes, homicides

Refugees: high TB rates; increased lead poisoning among children 
APPENDIX 


\section{REGIONS AND TOWNS}

\begin{tabular}{|c|c|c|c|}
\hline REGION & TOWN & COUNTY & CHNA \\
\hline BOSTON & $\begin{array}{l}\text { BOSTON } \\
\text { BROOKLINE } \\
\text { CHELSEA } \\
\text { REVERE } \\
\text { WINTHROP }\end{array}$ & $\begin{array}{l}\text { SUFFOLK } \\
\text { NORFOLK } \\
\text { SUFFOLK } \\
\text { SUFFOLK } \\
\text { SUFFOLK }\end{array}$ & $\begin{array}{l}\text { Alliance for Community Health } \\
\text { Alliance for Community Health } \\
\text { Alliance for Community Health } \\
\text { Alliance for Community Health } \\
\text { Alliance for Community Health }\end{array}$ \\
\hline CENTRAL & $\begin{array}{l}\text { ASHBURNHAM } \\
\text { ASHBY } \\
\text { AUBURN } \\
\text { AYER } \\
\text { BARRE } \\
\text { BELLINGHAM } \\
\text { BERLIN } \\
\text { BLACKSTONE } \\
\text { BOLTON } \\
\text { BOYLSTON } \\
\text { BRIMFIELD } \\
\text { BROOKFIELD } \\
\text { CHARLTON } \\
\text { CLINTON } \\
\text { DOUGLAS } \\
\text { DUDLEY } \\
\text { EAST BROOKFIELD } \\
\text { FITCHBURG } \\
\text { FRANKLIN } \\
\text { GARDNER } \\
\text { GRAFTON } \\
\text { GROTON } \\
\text { HARDWICK } \\
\text { HARVARD } \\
\text { HOLDEN } \\
\text { HOLLAND } \\
\text { HOPEDALE } \\
\text { HUBBARDSTON } \\
\text { LANCASTER } \\
\text { LEICESTER } \\
\text { LEOMINSTER } \\
\text { LUNENBURG } \\
\text { MEDWAY } \\
\text { MENDON } \\
\text { MILFORD } \\
\text { MILLBURY } \\
\text { MILLVILLE } \\
\text { NEW BRAINTREE } \\
\text { NORTH BROOKFIELD } \\
\text { NORTHBRIDGE } \\
\text { OAKHAM } \\
\text { OXFORD } \\
\text { OANA }\end{array}$ & $\begin{array}{l}\text { WORCESTER } \\
\text { MIDDLESEX } \\
\text { WORCESTER } \\
\text { MIDDLESEX } \\
\text { WORCESTER } \\
\text { NORFOLK } \\
\text { WORCESTER } \\
\text { WORCESTER } \\
\text { WORCESTER } \\
\text { WORCESTER } \\
\text { HAMPDEN } \\
\text { WORCESTER } \\
\text { WORCESTER } \\
\text { WORCESTER } \\
\text { WORCESTER } \\
\text { WORCESTER } \\
\text { WORCESTER } \\
\text { WORCESTER } \\
\text { NORFOLK } \\
\text { WORCESTER } \\
\text { WORCESTER } \\
\text { MIDDLESEX } \\
\text { WORCESTER } \\
\text { WORCESTER } \\
\text { WORCESTER } \\
\text { HAMPDEN } \\
\text { WORCESTER } \\
\text { WORCESTER } \\
\text { WORCESTER } \\
\text { WORCESTER } \\
\text { WORCESTER } \\
\text { WORCESTER } \\
\text { NORFOLK } \\
\text { WORCESTER } \\
\text { WORCESTER } \\
\text { WORCESTER } \\
\text { WORCESTER } \\
\text { WORCESTER } \\
\text { WORCESTER } \\
\text { WORCESTER } \\
\text { WORCESTER } \\
\text { WORCESTER } \\
\text { WOLPE }\end{array}$ & $\begin{array}{l}\text { Fitchburg/Gardner CHN } \\
\text { Fitchburg/Gardner CHN } \\
\text { Community Wellness Coalition } \\
\text { Fitchburg/Gardner CHN } \\
\text { Fitchburg/Gardner CHN } \\
\text { Community Partners for Health } \\
\text { Fitchburg/Gardner CHN } \\
\text { Community Partners for Health } \\
\text { Fitchburg/Gardner CHN } \\
\text { Community Wellness Coalition } \\
\text { CHN of Southern Worcester County } \\
\text { CHN of Southern Worcester County } \\
\text { CHN of Southern Worcester County } \\
\text { Fitchburg/Gardner CHN } \\
\text { Community Partners for Health } \\
\text { CHN of Southern Worcester County } \\
\text { CHN of Southern Worcester County } \\
\text { Fitchburg/Gardner CHN } \\
\text { Community Partners for Health } \\
\text { Fitchburg/Gardner CHN } \\
\text { Community Wellness Coalition } \\
\text { Fitchburg/Gardner CHN } \\
\text { Fitchburg/Gardner CHN } \\
\text { Fitchburg/Gardner CHN } \\
\text { Community Wellness Coalition } \\
\text { CHN of Southern Worcester County } \\
\text { Community Partners for Health } \\
\text { Fitchburg/Gardner CHN } \\
\text { Fitchburg/Gardner CHN } \\
\text { Community Wellness Coalition } \\
\text { Fitchburg/Gardner CHN } \\
\text { Fitchburg/Gardner CHN } \\
\text { Community Partners for Health } \\
\text { Community Partners for Health } \\
\text { Community Partners for Health } \\
\text { Community Wellness Coalition } \\
\text { Community Partners for Health } \\
\text { Fitchburg/Gardner CHN } \\
\text { CHN of Southern Worcester County } \\
\text { Community Partners for Health } \\
\text { Fitchburg/Gardner CHN } \\
\text { CHN of Southern Worcester County }\end{array}$ \\
\hline
\end{tabular}




\begin{tabular}{|c|c|c|c|}
\hline & $\begin{array}{l}\text { PAXTON } \\
\text { PEPPERELL } \\
\text { PRINCETON } \\
\text { RUTLAND } \\
\text { SHIRLEY } \\
\text { SHREWSBURY } \\
\text { SOUTHBRIDGE } \\
\text { SPENCER } \\
\text { STERLING } \\
\text { STURBRIDGE } \\
\text { SUTTON } \\
\text { TEMPLETON } \\
\text { TOWNSEND } \\
\text { UPTON } \\
\text { UXBRIDGE } \\
\text { WALES } \\
\text { WARREN } \\
\text { WEBSTER } \\
\text { WEST BOYLSTON } \\
\text { WEST BROOKFIELD } \\
\text { WESTMINSTER } \\
\text { WINCHENDON } \\
\text { WORCESTER } \\
\end{array}$ & $\begin{array}{l}\text { WORCESTER } \\
\text { MIDDLESEX } \\
\text { WORCESTER } \\
\text { WORCESTER } \\
\text { MIDDLESEX } \\
\text { WORCESTER } \\
\text { WORCESTER } \\
\text { WORCESTER } \\
\text { WORCESTER } \\
\text { WORCESTER } \\
\text { WORCESTER } \\
\text { WORCESTER } \\
\text { MIDDLESEX } \\
\text { WORCESTER } \\
\text { WORCESTER } \\
\text { HAMPDEN } \\
\text { WORCESTER } \\
\text { WORCESTER } \\
\text { WORCESTER } \\
\text { WORCESTER } \\
\text { WORCESTER } \\
\text { WORCESTER } \\
\text { WORCESTER }\end{array}$ & $\begin{array}{l}\text { Community Wellness Coalition } \\
\text { Fitchburg/Gardner CHN } \\
\text { Fitchburg/Gardner CHN } \\
\text { Fitchburg/Gardner CHN } \\
\text { Fitchburg/Gardner CHN } \\
\text { Community Wellness Coalition } \\
\text { CHN of Southern Worcester County } \\
\text { CHN of Southern Worcester County } \\
\text { Fitchburg/Gardner CHN } \\
\text { CHN of Southern Worcester County } \\
\text { Community Partners for Health } \\
\text { Fitchburg/Gardner CHN } \\
\text { Fitchburg/Gardner CHN } \\
\text { Community Partners for Health } \\
\text { Community Partners for Health } \\
\text { CHN of Southern Worcester County } \\
\text { CHN of Southern Worcester County } \\
\text { CHN of Southern Worcester County } \\
\text { Community Wellness Coalition } \\
\text { CHN of Southern Worcester County } \\
\text { Fitchburg/Gardner CHN } \\
\text { Fitchburg/Gardner CHN } \\
\text { Community Wellness Coalition }\end{array}$ \\
\hline $\begin{array}{c}\text { METRO } \\
\text { WEST }\end{array}$ & $\begin{array}{l}\text { ACTON } \\
\text { ARLINGTON } \\
\text { ASHLAND } \\
\text { BEDFORD } \\
\text { BELMONT } \\
\text { BOXBOROUGH } \\
\text { BRAINTREE } \\
\text { BURLINGTON } \\
\text { CAMBRIDGE } \\
\text { CANTON } \\
\text { CARLISLE } \\
\text { COHASSET } \\
\text { CONCORD } \\
\text { DEDHAM } \\
\text { DOVER } \\
\text { FOXBOROUGH } \\
\text { FRAMINGHAM } \\
\text { HINGHAM } \\
\text { HOLLISTON } \\
\text { HOPKINTON } \\
\text { HUDSON } \\
\text { HULL } \\
\text { LEXINGTON } \\
\text { LINCOLN } \\
\text { LITTLETON } \\
\text { MARLBOROUGH } \\
\text { MAYNARD }\end{array}$ & $\begin{array}{l}\text { MIDDLESEX } \\
\text { MIDDLESEX } \\
\text { MIDDLESEX } \\
\text { MIDDLESEX } \\
\text { MIDDLESEX } \\
\text { MIDDLESEX } \\
\text { NORFOLK } \\
\text { MIDDLESEX } \\
\text { MIDDLESEX } \\
\text { NORFOLK } \\
\text { MIDDLESEX } \\
\text { NORFOLK } \\
\text { MIDDLESEX } \\
\text { NORFOLK } \\
\text { NORFOLK } \\
\text { NORFOLK } \\
\text { MIDDLESEX } \\
\text { PLYMOUTH } \\
\text { MIDDLESEX } \\
\text { MIDDLESEX } \\
\text { MIDDLESEX } \\
\text { PLYMOUTH } \\
\text { MIDDLESEX } \\
\text { MIDDLESEX } \\
\text { MIDDLESEX } \\
\text { MIDDLESEX } \\
\text { MIDDLESEX }\end{array}$ & $\begin{array}{l}\text { Greater Woburn/Concord/Littleton CHN } \\
\text { Greater Cambridge/Somerville CHN } \\
\text { CHN of Greater Metro West } \\
\text { Greater Woburn/Concord/Littleton CHN } \\
\text { Greater Cambridge/Somerville CHN } \\
\text { Greater Woburn/Concord/Littleton CHN } \\
\text { Blue Hills Community Health Alliance } \\
\text { Greater Woburn/Concord/Littleton CHN } \\
\text { Greater Cambridge/Somerville CHN } \\
\text { Blue Hills Community Health Alliance } \\
\text { Greater Woburn/Concord/Littleton CHN } \\
\text { Blue Hills Community Health Alliance } \\
\text { Greater Woburn/Concord/Littleton CHN } \\
\text { West Suburban Health Network } \\
\text { West Suburban Health Network } \\
\mathrm{CHN} \text { of Greater Metro West } \\
\mathrm{CHN} \text { of Greater Metro West } \\
\text { Blue Hills Community Health Alliance } \\
\mathrm{CHN} \text { of Greater Metro West } \\
\mathrm{CHN} \text { of Greater Metro West } \\
\mathrm{CHN} \text { of Greater Metro West } \\
\text { Blue Hills Community Health Alliance } \\
\text { Greater Woburn/Concord/Littleton CHN } \\
\text { Greater Woburn/Concord/Littleton CHN } \\
\text { Greater Woburn/Concord/Littleton CHN } \\
\mathrm{CHN} \text { of Greater Metro West } \\
\mathrm{CHN} \text { of Greater Metro West }\end{array}$ \\
\hline
\end{tabular}




\begin{tabular}{|c|c|c|c|}
\hline & $\begin{array}{l}\text { MEDFIELD } \\
\text { MILLIS } \\
\text { MILTON } \\
\text { NATICK } \\
\text { NEEDHAM } \\
\text { NEWTON } \\
\text { NORFOLK } \\
\text { NORTHBOROUGH } \\
\text { NORWELL } \\
\text { NORWOOD } \\
\text { PLAINVILLE } \\
\text { QUINCY } \\
\text { RANDOLPH } \\
\text { SCITUATE } \\
\text { SHARON } \\
\text { SHERBORN } \\
\text { SOMERVILLE } \\
\text { SOUTHBOROUGH } \\
\text { STOW } \\
\text { SUDBURY } \\
\text { WALPOLE } \\
\text { WALTHAM } \\
\text { WATERTOWN } \\
\text { WAYLAND } \\
\text { WELLESLEY } \\
\text { WESTBOROUGH } \\
\text { WESTON } \\
\text { WESTWOOD } \\
\text { WEYMOUTH } \\
\text { WILMINGTON } \\
\text { WINCHESTER } \\
\text { WOBURN } \\
\text { WRENTHAM }\end{array}$ & $\begin{array}{l}\text { NORFOLK } \\
\text { NORFOLK } \\
\text { NORFOLK } \\
\text { MIDDLESEX } \\
\text { NORFOLK } \\
\text { MIDDLESEX } \\
\text { NORFOLK } \\
\text { WORCESTER } \\
\text { PLYMOUTH } \\
\text { NORFOLK } \\
\text { NORFOLK } \\
\text { NORFOLK } \\
\text { NORFOLK } \\
\text { PLYMOUTH } \\
\text { NORFOLK } \\
\text { MIDDLESEX } \\
\text { MIDDLESEX } \\
\text { WORCESTER } \\
\text { MIDDLESEX } \\
\text { MIDDLESEX } \\
\text { NORFOLK } \\
\text { MIDDLESEX } \\
\text { MIDDLESEX } \\
\text { MIDDLESEX } \\
\text { NORFOLK } \\
\text { WORCESTER } \\
\text { MIDDLESEX } \\
\text { NORFOLK } \\
\text { NORFOLK } \\
\text { MIDDLESEX } \\
\text { MIDDLESEX } \\
\text { MIDDLESEX } \\
\text { NORFOLK }\end{array}$ & $\begin{array}{l}\text { CHN of Greater Metro West } \\
\text { CHN of Greater Metro West } \\
\text { Blue Hills Community Health Alliance } \\
\text { CHN of Greater Metro West } \\
\text { West Suburban Health Network } \\
\text { West Suburban Health Network } \\
\text { CHN of Greater Metro West } \\
\text { CHN of Greater Metro West } \\
\text { Blue Hills Community Health Alliance } \\
\text { Blue Hills Community Health Alliance } \\
\text { CHN of Greater Metro West } \\
\text { Blue Hills Community Health Alliance } \\
\text { Blue Hills Community Health Alliance } \\
\text { Blue Hills Community Health Alliance } \\
\text { Blue Hills Community Health Alliance } \\
\text { CHN of Greater Metro West } \\
\text { Greater Cambridge/Somerville CHN } \\
\text { CHN of Greater Metro West } \\
\text { CHN of Greater Metro West } \\
\text { CHN of Greater Metro West } \\
\text { CHN of Greater Metro West } \\
\text { West Suburban Health Network } \\
\text { Greater Cambridge/Somerville CHN } \\
\text { CHN of Greater Metro West } \\
\text { West Suburban Health Network } \\
\text { CHN of Greater Metro West } \\
\text { West Suburban Health Network } \\
\text { West Suburban Health Network } \\
\text { Blue Hills Community Health Alliance } \\
\text { Greater Woburn/Concord/Littleton CHN } \\
\text { Greater Woburn/Concord/Littleton CHN } \\
\text { Greater Woburn/Concord/Littleton CHN } \\
\text { CHN of Greater Metro West }\end{array}$ \\
\hline NORTHEAST & $\begin{array}{l}\text { AMESBURY } \\
\text { ANDOVER } \\
\text { BEVERLY } \\
\text { BILLERICA } \\
\text { BOXFORD } \\
\text { CHELMSFORD } \\
\text { DANVERS } \\
\text { DRACUT } \\
\text { DUNSTABLE } \\
\text { ESSEX } \\
\text { EVERETT } \\
\text { GEORGETOWN } \\
\text { GLOUCESTER } \\
\text { GROVELAND } \\
\text { HAMILTON } \\
\text { HAVERHILL } \\
\text { IPSWICH }\end{array}$ & $\begin{array}{l}\text { ESSEX } \\
\text { ESSEX } \\
\text { ESSEX } \\
\text { MIDDLESEX } \\
\text { ESSEX } \\
\text { MIDDLESEX } \\
\text { ESSEX } \\
\text { MIDDLESEX } \\
\text { MIDDLESEX } \\
\text { ESSEX } \\
\text { MIDDLESEX } \\
\text { ESSEX } \\
\text { ESSEX } \\
\text { ESSEX } \\
\text { ESSEX } \\
\text { ESSEX } \\
\text { ESSEX }\end{array}$ & $\begin{array}{l}\text { Greater Haverhill CHN } \\
\text { Greater Lawrence CHN } \\
\text { Community Health Network North } \\
\text { Greater Lowell CHN } \\
\text { Greater Haverhill CHN } \\
\text { Greater Lowell CHN } \\
\text { North Shore CHN } \\
\text { Greater Lowell CHN } \\
\text { Greater Lowell CHN } \\
\text { Community Health Network North } \\
\text { North Suburban Health Alliance } \\
\text { Greater Haverhill CHN } \\
\text { Community Health Network North } \\
\text { Greater Haverhill CHN } \\
\text { Community Health Network North } \\
\text { Greater Haverhill CHN } \\
\text { Community Health Network North }\end{array}$ \\
\hline
\end{tabular}




\begin{tabular}{|c|c|c|c|}
\hline & $\begin{array}{l}\text { LAWRENCE } \\
\text { LOWELL } \\
\text { LYNN } \\
\text { LYNNFIELD } \\
\text { MALDEN } \\
\text { MANCHESTER } \\
\text { MARBLEHEAD } \\
\text { MEDFORD } \\
\text { MELROSE } \\
\text { MERRIMAC } \\
\text { METHUEN } \\
\text { MIDDLETON } \\
\text { NAHANT } \\
\text { NEWBURY } \\
\text { NEWBURYPORT } \\
\text { NORTH ANDOVER } \\
\text { NORTH READING } \\
\text { PEABODY } \\
\text { READING } \\
\text { ROCKPORT } \\
\text { ROWLEY } \\
\text { SALEM } \\
\text { SALISBURY } \\
\text { SAUGUS } \\
\text { STONEHAM } \\
\text { SWAMPSCOTT } \\
\text { TEWKSBURY } \\
\text { TOPSFIELD } \\
\text { TYNGSBOROUGH } \\
\text { WAKEFIELD } \\
\text { WENHAM } \\
\text { WEST NEWBURY } \\
\text { WESTFORD }\end{array}$ & $\begin{array}{l}\text { ESSEX } \\
\text { MIDDLESEX } \\
\text { ESSEX } \\
\text { ESSEX } \\
\text { MIDDLESEX } \\
\text { ESSEX } \\
\text { ESSEX } \\
\text { MIDDLESEX } \\
\text { MIDDLESEX } \\
\text { ESSEX } \\
\text { ESSEX } \\
\text { ESSEX } \\
\text { ESSEX } \\
\text { ESSEX } \\
\text { ESSEX } \\
\text { ESSEX } \\
\text { MIDDLESEX } \\
\text { ESSEX } \\
\text { MIDDLESEX } \\
\text { ESSEX } \\
\text { ESSEX } \\
\text { ESSEX } \\
\text { ESSEX } \\
\text { ESSEX } \\
\text { MIDDLESEX } \\
\text { ESSEX } \\
\text { MIDDLESEX } \\
\text { ESSEX } \\
\text { MIDDLESEX } \\
\text { MIDDLESEX } \\
\text { ESSEX } \\
\text { ESSEX } \\
\text { MIDDLESEX }\end{array}$ & $\begin{array}{l}\text { Greater Lawrence CHN } \\
\text { Greater Lowell CHN } \\
\text { North Shore CHN } \\
\text { North Shore CHN } \\
\text { North Suburban Health Alliance } \\
\text { Community Health Network North } \\
\text { North Shore CHN } \\
\text { North Suburban Health Alliance } \\
\text { North Suburban Health Alliance } \\
\text { Greater Haverhill CHN } \\
\text { Greater Lawrence CHN } \\
\text { Greater Lawrence CHN } \\
\text { North Shore CHN } \\
\text { Greater Haverhill CHN } \\
\text { Greater Haverhill CHN } \\
\text { Greater Lawrence CHN } \\
\text { North Suburban Health Alliance } \\
\text { North Shore CHN } \\
\text { North Suburban Health Alliance } \\
\text { Community Health Network North } \\
\text { Greater Haverhill CHN } \\
\text { North Shore CHN } \\
\text { Greater Haverhill CHN } \\
\text { North Shore CHN } \\
\text { North Suburban Health Alliance } \\
\text { North Shore CHN } \\
\text { Greater Lowell CHN } \\
\text { Community Health Network North } \\
\text { Greater Lowell CHN } \\
\text { North Suburban Health Alliance } \\
\text { Community Health Network North } \\
\text { Greater Haverhill CHN } \\
\text { Greater Lowell CHN }\end{array}$ \\
\hline SOUTHEAST & $\begin{array}{l}\text { ABINGTON } \\
\text { ACUSHNET } \\
\text { ATTLEBORO } \\
\text { AVON } \\
\text { BARNSTABLE } \\
\text { BERKLEY } \\
\text { BOURNE } \\
\text { BREWSTER } \\
\text { BRIDGEWATER } \\
\text { BROCKTON } \\
\text { CARVER } \\
\text { CHATHAM } \\
\text { CHILMARK } \\
\text { DARTMOUTH } \\
\text { DENNIS } \\
\text { DIGHTON } \\
\text { DUXBURY }\end{array}$ & $\begin{array}{l}\text { PLYMOUTH } \\
\text { BRISTOL } \\
\text { BRISTOL } \\
\text { NORFOLK } \\
\text { BARNSTABLE } \\
\text { BRISTOL } \\
\text { BARNSTABLE } \\
\text { BARNSTABLE } \\
\text { PLYMOUTH } \\
\text { PLYMOUTH } \\
\text { PLYMOUTH } \\
\text { BARNSTABLE } \\
\text { DUKES } \\
\text { BRISTOL } \\
\text { BARNSTABLE } \\
\text { BRISTOL } \\
\text { PLYMOUTH }\end{array}$ & $\begin{array}{l}\text { Greater Brockton CHN } \\
\text { Greater New Bedford CHN } \\
\text { Health \& Education Response } \\
\text { Greater Brockton CHN } \\
\text { Cape and Islands CHN } \\
\text { Health \& Education Response } \\
\text { Cape and Islands CHN } \\
\text { Cape and Islands CHN } \\
\text { Greater Brockton CHN } \\
\text { Greater Brockton CHN } \\
\text { South Shore Community Partners in Prevention } \\
\text { Cape and Islands CHN } \\
\text { Cape and Islands CHN } \\
\text { Greater New Bedford CHN } \\
\text { Cape and Islands CHN } \\
\text { Health \& Education Response } \\
\text { South Shore Community Partners in Prevention }\end{array}$ \\
\hline
\end{tabular}




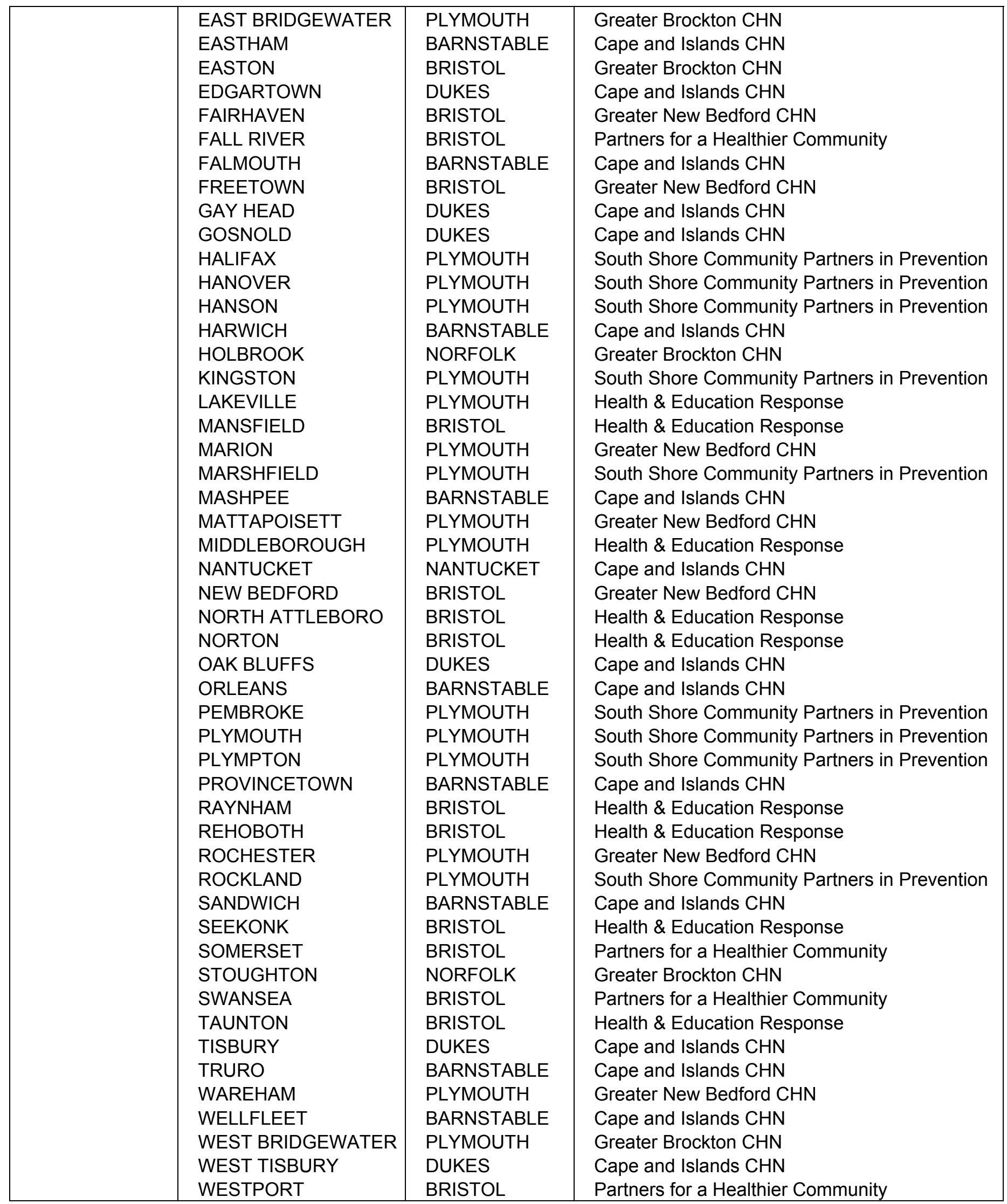




\begin{tabular}{|c|c|c|c|}
\hline & $\begin{array}{l}\text { WHITMAN } \\
\text { YARMOUTH }\end{array}$ & $\begin{array}{l}\text { PLYMOUTH } \\
\text { BARNSTABLE }\end{array}$ & $\begin{array}{l}\text { Greater Brockton } \mathrm{CHN} \\
\text { Cape and Islands } \mathrm{CHN}\end{array}$ \\
\hline WESTERN & 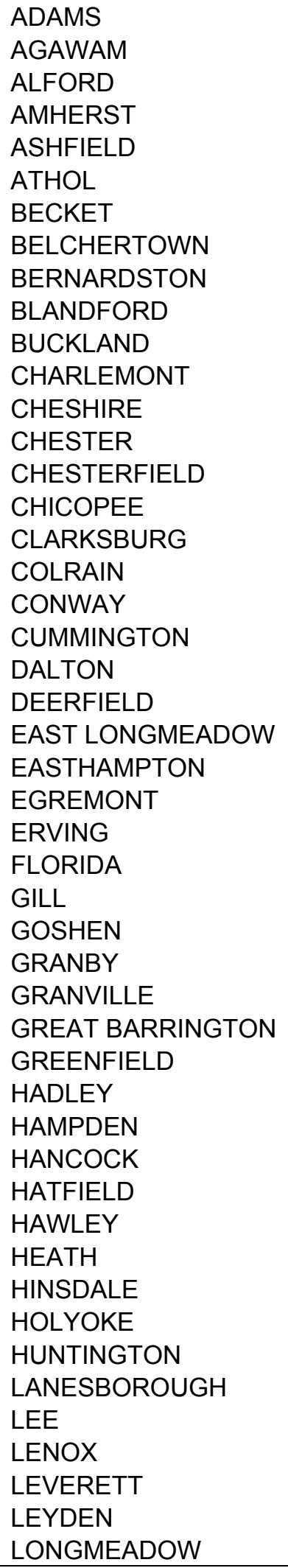 & $\begin{array}{l}\text { BERKSHIRE } \\
\text { HAMPDEN } \\
\text { BERKSHIRE } \\
\text { HAMPSHIRE } \\
\text { FRANKLIN } \\
\text { WORCESTER } \\
\text { BERKSHIRE } \\
\text { HAMPSHIRE } \\
\text { FRANKLIN } \\
\text { HAMPDEN } \\
\text { FRANKLIN } \\
\text { FRANKLIN } \\
\text { BERKSHIRE } \\
\text { HAMPDEN } \\
\text { HAMPSHIRE } \\
\text { HAMPDEN } \\
\text { BERKSHIRE } \\
\text { FRANKLIN } \\
\text { FRANKLIN } \\
\text { HAMPSHIRE } \\
\text { BERKSHIRE } \\
\text { FRANKLIN } \\
\text { HAMPDEN } \\
\text { HAMPSHIRE } \\
\text { BERKSHIRE } \\
\text { FRANKLIN } \\
\text { BERKSHIRE } \\
\text { FRANKLIN } \\
\text { HAMPSHIRE } \\
\text { HAMPSHIRE } \\
\text { HAMPDEN } \\
\text { BERKSHIRE } \\
\text { FRANKLIN } \\
\text { HAMPSHIRE } \\
\text { HAMPDEN } \\
\text { BERKSHIRE } \\
\text { HAMPSHIRE } \\
\text { FRANKLIN } \\
\text { FRANKLIN } \\
\text { BERKSHIRE } \\
\text { HAMPDEN } \\
\text { HAMPSHIRE } \\
\text { BERKSHIRE } \\
\text { BERKSHIRE } \\
\text { BERKSHIRE } \\
\text { FRANKLIN } \\
\text { HAMPLIN } \\
\text { HAMDN }\end{array}$ & $\begin{array}{l}\text { CHN of Berkshire } \\
\text { The Community Health Connection } \\
\text { CHN of Berkshire } \\
\text { Partnership for Health in Hampshire County } \\
\text { Upper Valley Health Web } \\
\text { Upper Valley Health Web } \\
\text { CHN of Berkshire } \\
\text { Partnership for Health in Hampshire County } \\
\text { Upper Valley Health Web } \\
\text { The Community Health Connection } \\
\text { Upper Valley Health Web } \\
\text { Upper Valley Health Web } \\
\text { CHN of Berkshire } \\
\text { Four (For) Communities } \\
\text { Partnership for Health in Hampshire County } \\
\text { Four (For) Communities } \\
\text { CHN of Berkshire } \\
\text { Upper Valley Health Web } \\
\text { Upper Valley Health Web } \\
\text { Partnership for Health in Hampshire County } \\
\text { CHN of Berkshire } \\
\text { Upper Valley Health Web } \\
\text { The Community Health Connection } \\
\text { Partnership for Health in Hampshire County } \\
\text { CHN of Berkshire } \\
\text { Upper Valley Health Web } \\
\text { CHN of Berkshire } \\
\text { Upper Valley Health Web } \\
\text { Partnership for Health in Hampshire County } \\
\text { Partnership for Health in Hampshire County } \\
\text { The Community Health Connection } \\
\text { CHN of Berkshire } \\
\text { Upper Valley Health Web } \\
\text { Partnership for Health in Hampshire County } \\
\text { The Community Health Connection } \\
\text { CHN of Berkshire } \\
\text { Partnership for Health in Hampshire County } \\
\text { Upper Valley Health Web } \\
\text { Upper Valley Health Web } \\
\text { CHN of Berkshire } \\
\text { Four (For) Communities } \\
\text { Four (For) Communities } \\
\text { CHN of Berkshire } \\
\text { CHN of Berkshire } \\
\text { CHN of Berkshire } \\
\text { Upper Valley Health Web } \\
\text { Upper Valley Health Web } \\
\text { The Community Health Connection } \\
\text { There }\end{array}$ \\
\hline
\end{tabular}




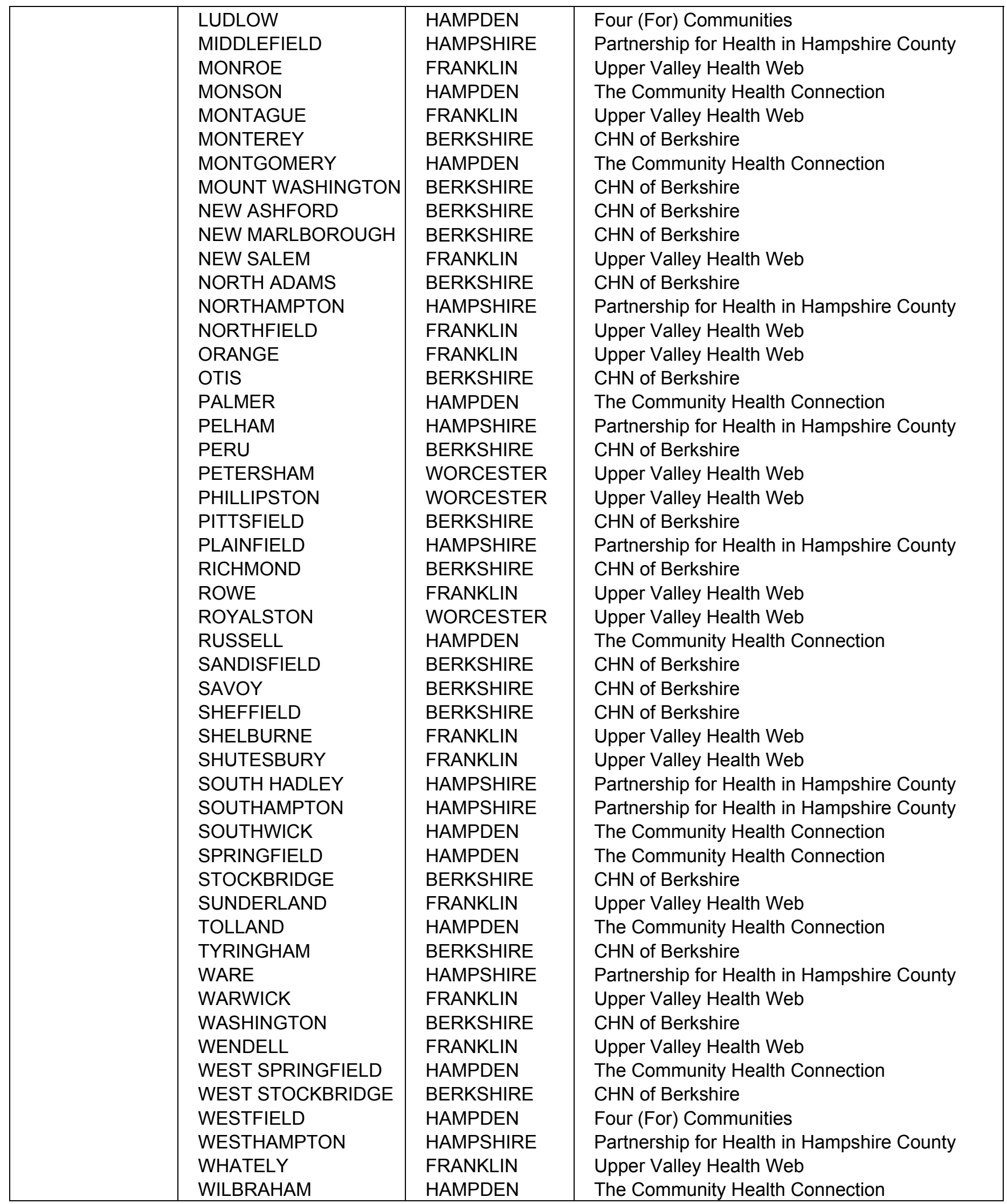




\begin{tabular}{|l|l|l|l|}
\hline & WILLIAMSBURG & HAMPSHIRE & Partnership for Health in Hampshire County \\
& WILLIAMSTOWN & BERKSHIRE & CHN of Berkshire \\
& WINDSOR & BERKSHIRE & CHN of Berkshire \\
& WORTHINGTON & HAMPSHIRE & Partnership for Health in Hampshire County \\
\hline
\end{tabular}




\section{DATA SOURCES}

Massachusetts Division of Health Care Finance and Policy

MDPH, Bureau of Communicable Disease Control

MDPH, Bureau of Substance Abuse Services

MDPH, Environmental Health Bureau

MDPH, Health Information, Statistics, Research \& Evaluation Bureau

SAMHSA, Office of Applied Studies 